\title{
Stereodivergent Coupling of 1,3-Dienes with Aldimine Esters Enabled by Synergistic Pd and Cu Catalysis
}

Qinglong Zhang, ${ }^{\ddagger}$ Huimin Yu, ${ }^{\ddagger}$ Lulu Shen, Tianhua Tang, Dongfang Dong, Weiwei Chai and Weiwei $\mathrm{Zi}^{*}$

State Key Laboratory and Institute of Elemento-Organic Chemistry, College of Chemistry, Nankai

University, Tianjin 300071, China

Table of Contents

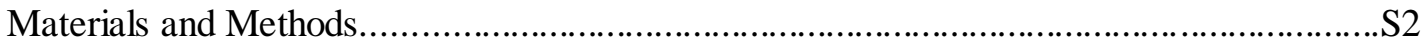

Preparation and Characterization of Substrates .....................................................S2

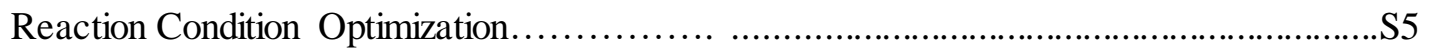

General Procedure for Coupling of 1,3-Dienes and Aldimine Esters................................S8

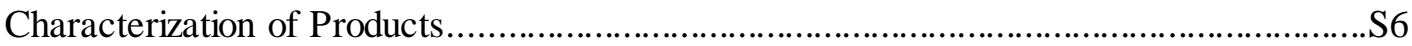

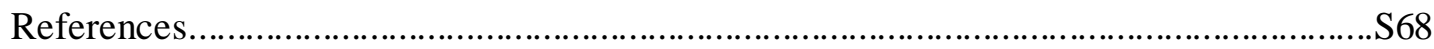

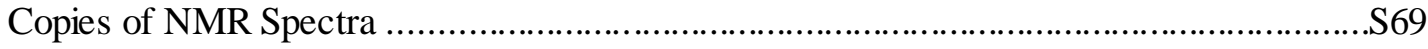




\section{Materials and Methods}

Unless otherwise noted, all reactions were assembled on a Schlenk vacuum line or in a glovebox using oven-dried glassware and were stirred with Teflon-coated magnetic stirring bars. All the ligands were purchased from Strem Chemicals or Sinocompound Catalysts Co., Ltd. and were used as received. Unless otherwise noted, reagents were obtained from commercial sources and used without further purification. THF, $\mathrm{Et}_{2} \mathrm{O}$, toluene were distilled with $\mathrm{Na}$ before using. Dichloromethane was distilled with $\mathrm{CaH}_{2}$ before using. All other dried solvents (DMF, $\mathrm{CH}_{3} \mathrm{CN}, \mathrm{PhH}, 1$,4-dioxane, Et $3 \mathrm{~N}$, DIPEA) were purchased from MERYER (Shanghai) Chemical Technology Co., Ltd. TLC analys is of reaction mixtures was performed on huanghai silica gel 60 F254 TLC plates and visualized by $\mathrm{UV}, \mathrm{I}_{2} /$ silica, and/or ceric ammonium molybdate stain. All work-up and purification procedures were carried out with reagent grade solvents in air. Reaction temperatures above $23{ }^{\circ} \mathrm{C}$ refer to temperatures of an aluminum heating block or a silicon oil bath, which were controlled by an electronic temperature modulator from IKA. Flash chromatography was carried out with silica gel $200-300$ or $300-400$ mesh. ${ }^{1} \mathrm{H}$ and ${ }^{13} \mathrm{C}$ NMR spectra were recorded with Bruker AV-400 spectrometers and were referenced to residual ${ }^{1} \mathrm{H}$ and ${ }^{13} \mathrm{C}$ signals of the deuterated solvents respectively $(\delta \mathrm{H} \mathrm{7.26, \delta} \mathrm{C} 77.00$ for chloroform). Abbreviations are as follows: s (singlet), d (doublet), t (triplet), $\mathrm{q}$ (quartet), m (multiplet), br (broad). The ee value was determined on Shimadzu LC-20A HPLC workstation (Daicel chiral columns Chiralpak IA, IC, ID, IE, IF (4.6 x $250 \mathrm{~mm}$ )). HRMS was performed on a Varian QFT-ESI instrumental. Optical rotation were determined on a IP-digi 300/2 apparatus. Melting points were determined on an X-4B melting point apparatus.

\section{Preparation and Characterization of Substrates}

All 1,3-dienes used in this work were known compounds and were prepared according to literature procedure ${ }^{1}$. The aldimine esters were prepared according to literature methods ${ }^{2}$. Among the aldimine esters used in this work, $\mathbf{2 c}, \mathbf{2 d}, \mathbf{2 e}, \mathbf{2 f}, \mathbf{2 g}, \mathbf{2 i}, \mathbf{2 j}, \mathbf{2 p}$ were prepared for the first time and their characterization were given as below.

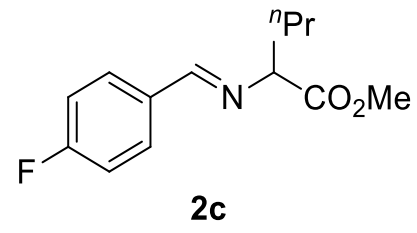

methyl $(E)$-2-((4-fluorobenzylidene)amino)pentanoate

${ }^{1} \mathbf{H}$ NMR $\left(400 \mathrm{MHz}, \mathbf{C D C l}_{3}\right) \delta 8.22(\mathrm{~s}, 1 \mathrm{H}), 7.84-7.72(\mathrm{~m}, 2 \mathrm{H}), 7.16-7.02(\mathrm{~m}, 2 \mathrm{H}), 3.97(\mathrm{dd}, J=$ $8.4,5.4 \mathrm{~Hz}, 1 \mathrm{H}), 3.73(\mathrm{~s}, 3 \mathrm{H}), 2.03-1.79(\mathrm{~m}, 2 \mathrm{H}), 1.43-1.21(\mathrm{~m}, 2 \mathrm{H}), 0.92(\mathrm{t}, J=7.4 \mathrm{~Hz}, 3 \mathrm{H})$.

${ }^{13}$ C NMR (101 MHz, CDCl $) \delta$ 172.74, 164.55 (d, $\left.J=251.3 \mathrm{~Hz}\right), 161.70,131.98$ (d, $\left.J=3.1 \mathrm{~Hz}\right)$, $130.50(\mathrm{~d}, J=8.7 \mathrm{~Hz}), 115.70(\mathrm{~d}, J=22.0 \mathrm{~Hz}), 73.18,52.14,35.44,19.15,13.68$. 
${ }^{19}$ F NMR (376 MHz, $\left.\mathrm{CDCl}_{3}\right) \delta-113.21$

HRMS (ESI) calcd. for $\mathrm{C}_{13} \mathrm{H}_{17} \mathrm{FNO}_{2}{ }^{+}(\mathrm{M}+\mathrm{H})^{+}:$238.1243, Found: 238.1234<smiles>CCCCC(/N=C/c1ccc(F)cc1)C(C)=O</smiles>

(E)-methyl 2-((4-fluorobenzylidene)amino)hexanoate

${ }^{1}$ H NMR (400 MHz, CDCl 3 ) $\delta 8.24(\mathrm{~s}, 1 \mathrm{H}), 7.79(\mathrm{dd}, J=7.4,5.7 \mathrm{~Hz}, 2 \mathrm{H}), 7.10(\mathrm{t}, J=8.1 \mathrm{~Hz}, 2 \mathrm{H})$, $3.97(\mathrm{dd}, J=7.9,5.7 \mathrm{~Hz}, 1 \mathrm{H}), 3.75(\mathrm{~s}, 3 \mathrm{H}), 2.04-1.96(\mathrm{~m}, 1 \mathrm{H}), 1.93-1.83(\mathrm{~m}, 1 \mathrm{H}), 1.46-1.15(\mathrm{~m}$, $4 \mathrm{H}), 0.89(\mathrm{t}, J=6.9 \mathrm{~Hz}, 3 \mathrm{H})$.

${ }^{13}$ C NMR (101 MHz, CDCl $) \delta 172.75,164.55(\mathrm{~d}, J=251.4 \mathrm{~Hz}), 161.71,131.97(\mathrm{~d}, J=3.1 \mathrm{~Hz})$, $130.51(\mathrm{~d}, J=8.7 \mathrm{~Hz}), 115.71(\mathrm{~d}, J=21.9 \mathrm{~Hz}), 73.48,52.15,33.15,28.09,22.35,13.94$.

${ }^{19}$ F NMR (376 MHz, CDCl 3 ) $\delta-113.19$

HRMS (ESI) calcd. for $\mathrm{C}_{14} \mathrm{H}_{19} \mathrm{FNO}_{2}{ }^{+}(\mathrm{M}+\mathrm{H})^{+}: 252.1400$, Found: 252.1389<smiles>CC(=O)C(CCc1ccccc1)/N=C/c1ccc(F)cc1</smiles>

(E)-methyl 2-((4-fluorobenzylidene)amino)-4-phenylbutanoate

${ }^{1}$ H NMR (400 MHz, CDCl 3 ) $\delta 8.20(\mathrm{~s}, 1 \mathrm{H}), 7.83$ - $7.75(\mathrm{~m}, 2 \mathrm{H}), 7.31$ - $7.24(\mathrm{~m}, 2 \mathrm{H}), 7.22-7.14(\mathrm{~m}$, $3 \mathrm{H}), 7.14-7.07(\mathrm{~m}, 2 \mathrm{H}), 3.99(\mathrm{dd}, J=8.5,5.1 \mathrm{~Hz}, 1 \mathrm{H}), 3.73(\mathrm{~s}, 3 \mathrm{H}), 2.70(\mathrm{ddd}, J=14.7,9.4,5.7 \mathrm{~Hz}$, $1 \mathrm{H}), 2.63-2.53(\mathrm{~m}, 1 \mathrm{H}), 2.41-2.30(\mathrm{~m}, 1 \mathrm{H}), 2.30-2.19(\mathrm{~m}, 1 \mathrm{H})$.

${ }^{13}$ C NMR (101 MHz, CDCl 3 ) $\delta 172.41,164.65(\mathrm{~d}, J=251.7 \mathrm{~Hz}), 162.49,140.89,131.93$ (d, $J=2.8$ $\mathrm{Hz}), 130.58$ (d, $J=8.7 \mathrm{~Hz}), 128.53,128.48,115.79$ (d, $J=22.0 \mathrm{~Hz}), 115.79$ (d, $J=22.0 \mathrm{~Hz}), 72.35$, $52.25,34.54,31.96$.

${ }^{19}$ F NMR (376 MHz, $\left.\mathbf{C D C l}_{3}\right) \delta-112.99$

HRMS (ESI) calcd. for $\mathrm{C}_{18} \mathrm{H}_{19} \mathrm{FNO}_{2}{ }^{+}(\mathrm{M}+\mathrm{H})^{+}: 300.1400$, Found: 300.1389<smiles>CCCC(Cc1ccccc1)C(C)=O</smiles>

methyl (E)-2-((4-fluorobenzylidene)amino)-3-phenylpropanoate 
${ }^{1}$ H NMR (400 MHz, CDCl 3 ) $\delta 7.85(\mathrm{~s}, 1 \mathrm{H}), 7.71-7.64(\mathrm{~m}, 2 \mathrm{H}), 7.25$ - $7.13(\mathrm{~m}, 5 \mathrm{H}), 7.10$ - $7.02(\mathrm{~m}$, $2 \mathrm{H}), 4.15(\mathrm{dd}, J=8.9,5.0 \mathrm{~Hz}, 1 \mathrm{H}), 3.75(\mathrm{~s}, 3 \mathrm{H}), 3.36(\mathrm{dd}, J=13.5,5.0 \mathrm{~Hz}, 1 \mathrm{H}), 3.13(\mathrm{dd}, J=13.5$, $8.9 \mathrm{~Hz}, 1 \mathrm{H})$.

${ }^{13}$ C NMR (101 MHz, CDCl 3 ) $\delta 172.09,164.56(\mathrm{~d}, J=251.3 \mathrm{~Hz}), 162.34,137.33,131.84(\mathrm{~d}, J=3.0$ $\mathrm{Hz}), 130.44(\mathrm{~d}, J=8.8 \mathrm{~Hz}), 129.76,128.35,126.63,115.68(\mathrm{~d}, J=21.8 \mathrm{~Hz}), 74.90,52.31,39.79$.

${ }^{19} \mathrm{~F}$ NMR (376 MHz, $\left.\mathrm{CDCl}_{3}\right) \delta-113.14$

HRMS (ESI) calcd. for $\mathrm{C}_{17} \mathrm{H}_{17} \mathrm{FNO}_{2}{ }^{+}(\mathrm{M}+\mathrm{H})^{+}:$286.1243, Found: 286.1234<smiles>CC(=O)CC(/N=C/c1ccc(F)cc1)C(C)=O</smiles>

dimethyl $(E)$-2-((4-fluorobenzylidene)amino)succinate

${ }^{1}$ H NMR (400 MHz, CDCl $) \delta 8.35(\mathrm{~s}, 1 \mathrm{H}), 7.81-7.73(\mathrm{~m}, 2 \mathrm{H}), 7.17-7.02(\mathrm{~m}, 2 \mathrm{H}), 4.47$ (dd, $J=$ 7.7, $5.8 \mathrm{~Hz}, 1 \mathrm{H}), 3.75(\mathrm{~s}, 3 \mathrm{H}), 3.67(\mathrm{~s}, 3 \mathrm{H}), 3.15(\mathrm{dd}, J=16.8,5.8 \mathrm{~Hz}, 1 \mathrm{H}), 2.87(\mathrm{dd}, J=16.8,7.8 \mathrm{~Hz}$, $1 \mathrm{H})$.

${ }^{13}$ C NMR (101 MHz, CDCl 3$) \delta 171.26,171.10,164.70(\mathrm{~d}, \mathrm{~J}=253.5 \mathrm{~Hz}), 163.91,131.79(\mathrm{~d}, J=3.2$ $\mathrm{Hz}), 130.67(\mathrm{~d}, J=8.8 \mathrm{~Hz}), 115.74(\mathrm{~d}, J=22.0 \mathrm{~Hz}), 68.74,52.59,51.85,37.31$.

${ }^{19}$ F NMR (376 MHz, CDCl 3 ) $\delta-112.69$

HRMS (ESI) calcd. for $\mathrm{C}_{13} \mathrm{H}_{15} \mathrm{FNO}_{4}{ }^{+}(\mathrm{M}+\mathrm{H})^{+}: 268.0985$, Found: 268.0975<smiles>CSCCC(/N=C/c1ccc(F)cc1)C(C)=O</smiles>

methyl (E)-2-((4-fluorobenzylidene)amino)-4-(methylthio)butanoate

${ }^{1}$ H NMR (400 MHz, $\left.\mathbf{C D C l}_{3}\right) \delta 8.29(\mathrm{~s}, 1 \mathrm{H}), 7.86-7.75(\mathrm{~m}, 2 \mathrm{H}), 7.15-7.07$ (m, 2H), 4.20 (dd, $J=$ $8.5,5.0 \mathrm{~Hz}, 1 \mathrm{H}), 3.75(\mathrm{~s}, 3 \mathrm{H}), 2.58(\mathrm{ddd}, J=13.0,7.5,5.4 \mathrm{~Hz}, 1 \mathrm{H}), 2.44(\mathrm{dt}, J=13.1,7.8 \mathrm{~Hz}, 1 \mathrm{H})$, $2.35-2.18(\mathrm{~m}, 2 \mathrm{H}), 2.09(\mathrm{~s}, 3 \mathrm{H})$.

${ }^{13}$ C NMR (101 MHz, CDCl $) \delta 172.18,164.65(\mathrm{~d}, J=251.7 \mathrm{~Hz}), 162.92,131.85(\mathrm{~d}, J=3.2 \mathrm{~Hz})$, $130.58(\mathrm{~d}, J=8.7 \mathrm{~Hz}), 115.76(\mathrm{~d}, J=21.9 \mathrm{~Hz}), 71.06,52.32,31.90,30.23,15.19$.

${ }^{19}$ F NMR (376 MHz, $\left.\mathbf{C D C l}_{3}\right) \delta-112.86$

HRMS (ESI) calcd. for $\mathrm{C}_{13} \mathrm{H}_{17} \mathrm{FNO}_{2} \mathrm{~S}^{+}(\mathrm{M}+\mathrm{H})^{+}:$270.0964, Found: 270.0955 
<smiles>C=CCC(/N=C/c1ccc(F)cc1)C(C)=O</smiles>

2j

methyl $(E)$-2-((4-fluorobenzylidene)amino)pent-4-enoate

${ }^{1} \mathbf{H}$ NMR $\left(400 \mathrm{MHz}, \mathbf{C D C l}_{3}\right) \delta 8.22(\mathrm{~s}, 1 \mathrm{H}), 7.83-7.74(\mathrm{~m}, 2 \mathrm{H}), 7.10(\mathrm{t}, J=8.7 \mathrm{~Hz}, 2 \mathrm{H}), 5.74(\mathrm{ddt}, J$ $=17.2,10.1,7.1 \mathrm{~Hz}, 1 \mathrm{H}), 5.18-5.01(\mathrm{~m}, 2 \mathrm{H}), 4.04(\mathrm{dd}, J=8.0,5.6 \mathrm{~Hz}, 1 \mathrm{H}), 3.75(\mathrm{~s}, 3 \mathrm{H}), \delta 2.84-$

$2.73(\mathrm{~m}, 1 \mathrm{H}), 2.68-2.56(\mathrm{~m}, 1 \mathrm{H})$.

${ }^{13}$ C NMR $\left(101 \mathrm{MHz}, \mathbf{C D C l}_{3}\right) \delta 172.01,164.61(\mathrm{~d}, J=251.6 \mathrm{~Hz}), 162.19,133.67,131.89(\mathrm{~d}, J=2.9$ $\mathrm{Hz}), 130.55(\mathrm{~d}, J=8.7 \mathrm{~Hz}), 118.33,115.72(\mathrm{~d}, J=21.9 \mathrm{~Hz}), 72.99,52.21,37.81$.

${ }^{19}$ F NMR (376 MHz, $\left.\mathrm{CDCl}_{3}\right) \delta-113.07$

HRMS (ESI) calcd. for $\mathrm{C}_{13} \mathrm{H}_{15} \mathrm{FNO}_{2}{ }^{+}(\mathrm{M}+\mathrm{H})^{+}: 236.1087$, Found: 236.1077<smiles>CCCCC(C)C(=O)N=Cc1ccc(F)cc1</smiles>

tert-butyl $(E)$-2-((4-fluorobenzylidene)amino)propanoate

${ }^{1} \mathrm{H}$ NMR $\left(400 \mathrm{MHz}, \mathbf{C D C l}_{3}\right) \delta 8.26(\mathrm{~d}, J=1.6 \mathrm{~Hz}, 1 \mathrm{H}), 7.78(\mathrm{dd}, J=7.9,5.6 \mathrm{~Hz}, 2 \mathrm{H}), 7.09(\mathrm{td}, J=$ $8.6,1.8 \mathrm{~Hz}, 2 \mathrm{H}), 4.03(\mathrm{qd}, J=6.8,1.7 \mathrm{~Hz}, 1 \mathrm{H}), 1.49(\mathrm{~s}, 3 \mathrm{H}), 1.47$ (s, 9H).

${ }^{13}$ C NMR (101 MHz, CDCl $) \delta 171.75,164.47(\mathrm{~d}, J=251.0 \mathrm{~Hz}), 161.00,132.29(\mathrm{~d}, J=3.1 \mathrm{~Hz})$, $130.36(\mathrm{~d}, J=8.7 \mathrm{~Hz}), 115.64(\mathrm{~d}, J=22.0 \mathrm{~Hz}), 81.16,68.33,28.04,19.40$.

${ }^{19}$ F NMR (376 MHz, $\left.\mathbf{C D C l}_{3}\right) \delta-113.57$

HRMS (ESI) calcd. for $\mathrm{C}_{14} \mathrm{H}_{19} \mathrm{FNO}_{2}{ }^{+}(\mathrm{M}+\mathrm{H})^{+}: 252.1400$, Found: 252.1390

\section{Reaction Condition Optimization}

Table S1. Investigation of the Bases ${ }^{a}$
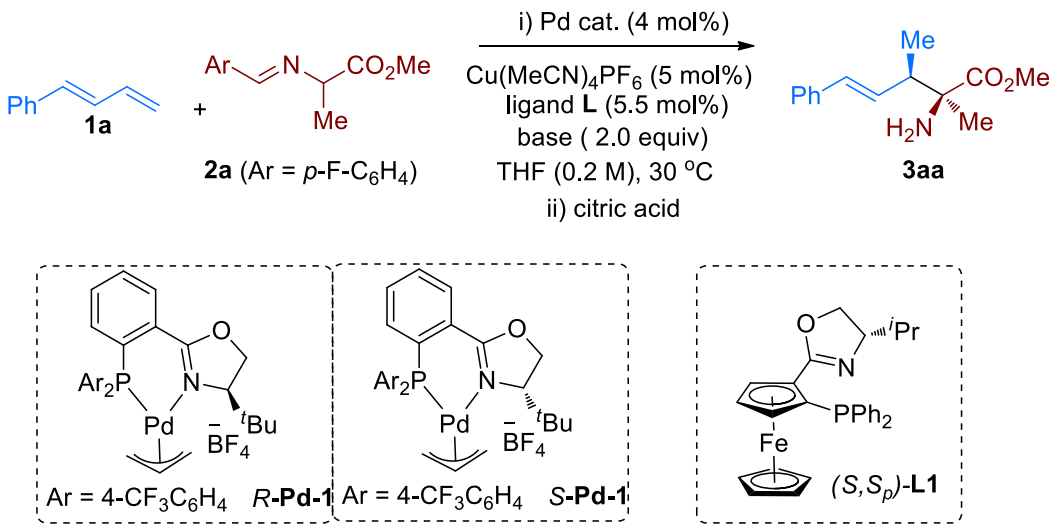


\begin{tabular}{cccccc}
\hline entry & Pd cat. & Base & ${\mathrm{yield}(\%)^{b}}^{2}$ & $\mathrm{dr}^{b}$ & $\mathrm{ee}(\%)^{c}$ \\
\hline 1 & $R$-Pd-1 & $\mathrm{Et}_{3} \mathrm{~N}$ & 53 & $1.6: 1$ & $95 / 67$ \\
2 & $R$-Pd-1 & ${ }^{i} \mathrm{Pr}_{2} \mathrm{NEt}$ & trace & - & $-/-$ \\
3 & $R$-Pd-1 & $\mathrm{DBU}$ & $\mathrm{NR}$ & - & $-/-$ \\
4 & $R$-Pd-1 & $\mathrm{DABCO}$ & $\mathrm{NR}$ & - & $-/-$ \\
5 & $R$-Pd-1 & $\mathrm{Cs}_{2} \mathrm{CO}_{3}$ & trace & - & $-/-$ \\
6 & $S$-Pd-1 & $\mathrm{Et}_{3} \mathrm{~N}$ & 50 & $1: 1.3$ & $40 / 93$ \\
\hline
\end{tabular}

${ }^{a}$ Reaction conditions: i) 1a $(0.2 \mathrm{mmol}), \mathbf{2 a}(0.1 \mathrm{mmol}), \mathrm{Pd}$ cat. $(4 \mathrm{~mol} \%), \mathrm{Cu}(\mathrm{MeCN})_{4} \mathrm{PF}_{6}(5 \mathrm{~mol} \%),\left(S, S_{p}\right)-\mathbf{L}$ or $\left(R, R_{p}\right)$-L $(5.5 \mathrm{~mol} \%)$, base $(200 \mathrm{~mol} \%)$, THF $(0.5 \mathrm{~mL}), 30{ }^{\circ} \mathrm{C}, 36 \mathrm{~h}$; ii) citric acid $(10 \%, 4 \mathrm{~mL}) .{ }^{b}$ Determined by ${ }^{1} \mathrm{H}$ NMR analy sis of the crude product. NR, no reaction. ${ }^{c}$ Determined by HPLC.

\section{Table S2. Investigation of the Ligands for $\mathrm{Cu}^{a}$}
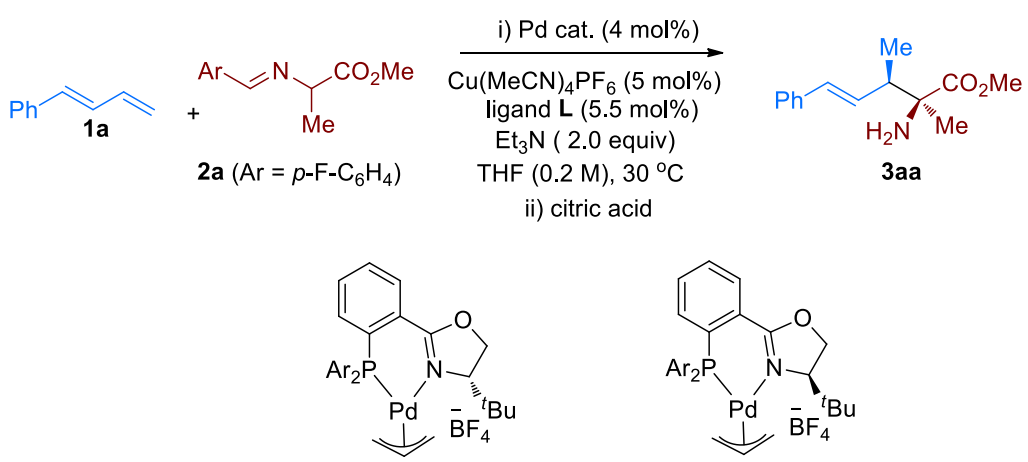

$\mathrm{Ar}=4-\mathrm{CF}_{3} \mathrm{C}_{6} \mathrm{H}_{4} \quad \mathrm{~S}-\mathrm{Pd}-1$

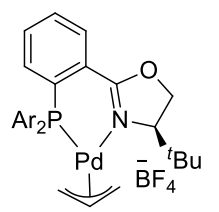

$\mathrm{Ar}=4-\mathrm{CF}_{3} \mathrm{C}_{6} \mathrm{H}_{4} \quad R-\mathrm{Pd}-1$
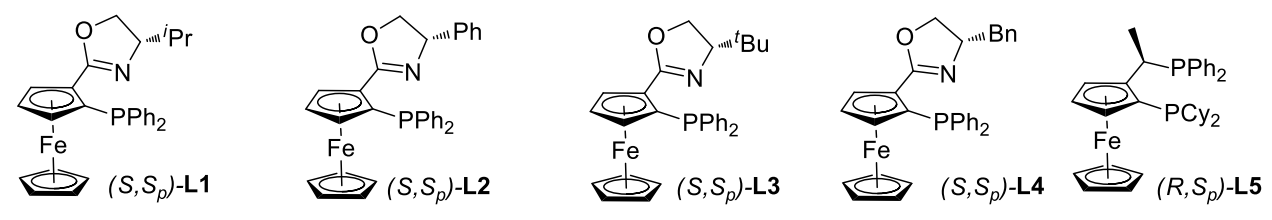

\begin{tabular}{cccccc}
\hline entry & Pd cat. & Cu ligand $\mathbf{L}$ & $\mathrm{yield}(\%)^{b}$ & $\mathrm{dr}^{b}$ & ee $(\%)^{c}$ \\
\hline 1 & $S$-Pd-1 & $\left(S, S_{p}\right)$-L1 & 50 & $1: 1.3$ & $40 / 93$ \\
2 & $S$-Pd-1 & $\left(S, S_{p}\right)$-L2 & 73 & $1: 2.7$ & $66 / 95$ \\
3 & $S$-Pd-1 & $\left(S, S_{p}\right)$-L3 & 61 & $1: 2.5$ & $10 / 90$ \\
4 & $S$-Pd-1 & $\left(S, S_{p}\right)$-LA & 40 & $1: 1.4$ & $30 / 50$ \\
5 & $S$-Pd-1 & $\left(S, S_{p}\right)-\mathbf{L} 5$ & $<10$ & $1: 1.6$ & $-/-$ \\
$\mathbf{6}$ & $\boldsymbol{R}$-Pd-1 & $\left(S, S_{p}\right)-\mathbf{L} 2$ & 75 & $2: 1$ & $95 / 83$ \\
\hline
\end{tabular}

Reaction conditions: i) 1a $(0.2 \mathrm{mmol}), \mathbf{2 a}(0.1 \mathrm{mmol}), \mathrm{Pd}$ cat. $(4 \mathrm{~mol} \%), \mathrm{Cu}(\mathrm{MeCN})_{4} \mathrm{PF}_{6}(5 \mathrm{~mol} \%), \mathbf{L}(5.5 \mathrm{~mol} \%)$, $\mathrm{Et}_{3} \mathrm{~N}(200 \mathrm{~mol} \%)$, THF $(0.5 \mathrm{~mL}), 30{ }^{\circ} \mathrm{C}, 36 \mathrm{~h}$; ii) citric acid $(10 \%, 4 \mathrm{~mL}) .{ }^{b}$ Determined by ${ }^{1} \mathrm{H}$ NMR analysis of the crude product. NR, no reaction. ${ }^{c}$ Determined by HPLC. 
Table S3. Investigation of the Solvents ${ }^{a}$

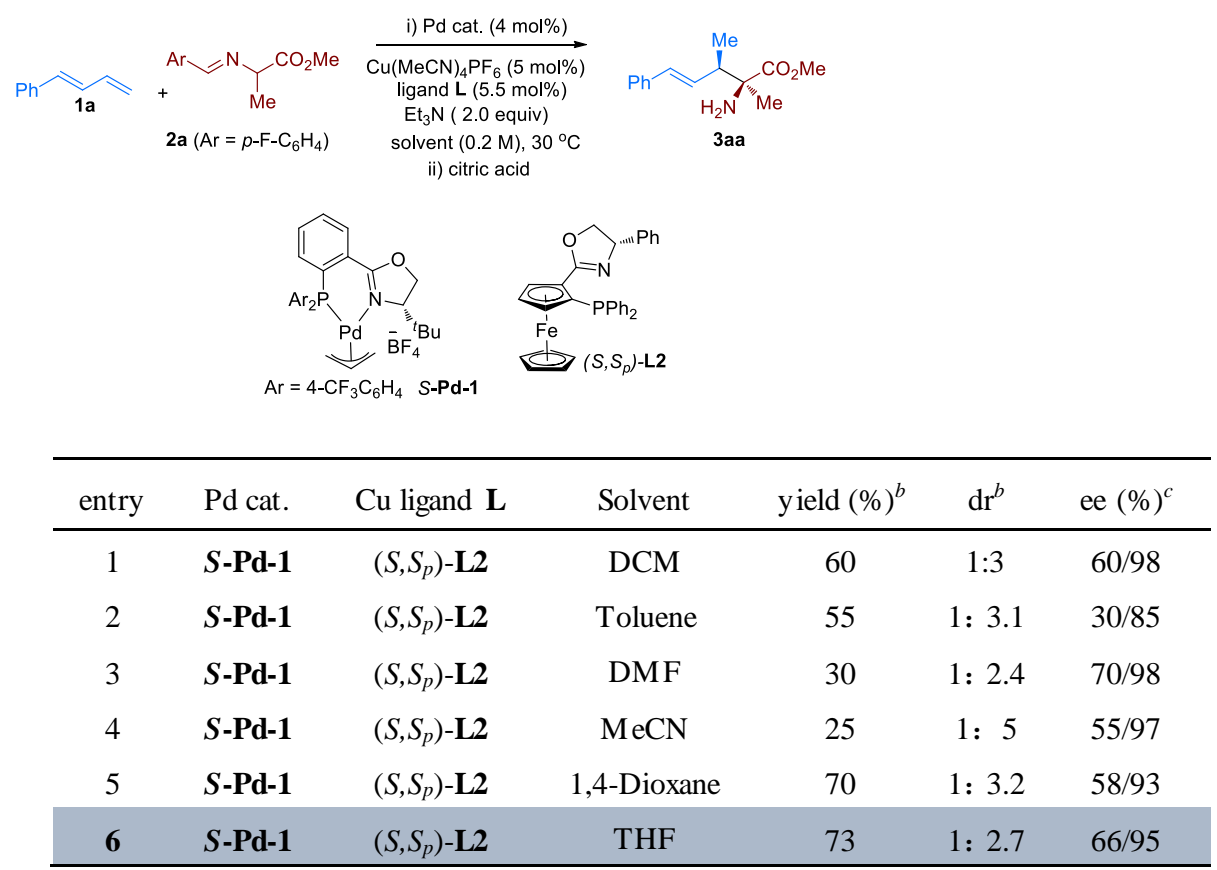

${ }^{a}$ Reaction conditions: i) 1a $(0.2 \mathrm{mmol}), \mathbf{2 a}(0.1 \mathrm{mmol}), \boldsymbol{S}$-Pd-1 $(4 \mathrm{~mol} \%), \mathrm{Cu}(\mathrm{MeCN})_{4} \mathrm{PF}_{6}(5 \mathrm{~mol} \%),\left(S, S_{p}\right)-\mathbf{L} 2(5.5$ mol\%), Et $3 \mathrm{~N}(200 \mathrm{~mol} \%)$, solvent $(0.5 \mathrm{~mL}), 30{ }^{\circ} \mathrm{C}, 36 \mathrm{~h}$; ii) citric acid $(10 \%, 4 \mathrm{~mL}) .{ }^{b}$ Determined by ${ }^{1} \mathrm{H} \mathrm{NMR}$ analy sis of the crude product. NR, no reaction. ${ }^{c}$ Determined by HPLC.

\section{Table S4. Investigation of the Ligands for $\operatorname{Pd}^{a}$}
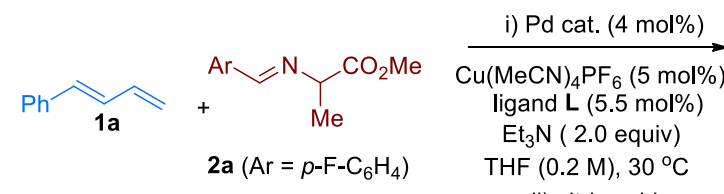
ii) citric acid

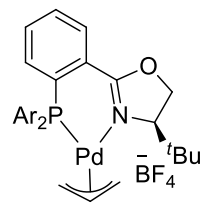

$\mathrm{Ar}=4-\mathrm{CF}_{3} \mathrm{C}_{6} \mathrm{H}_{4} \quad \mathbf{P d}-1$
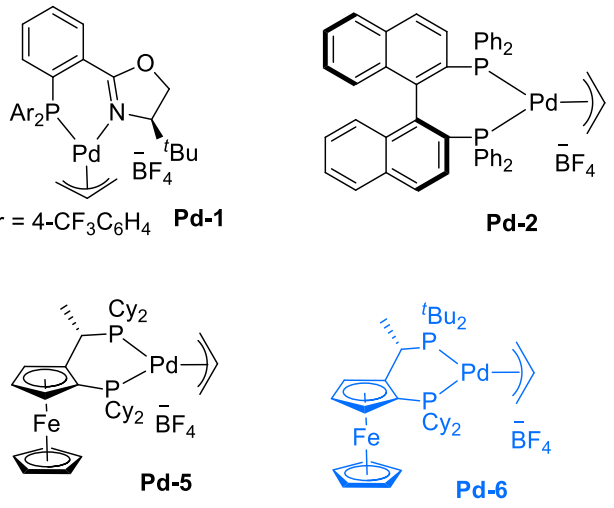
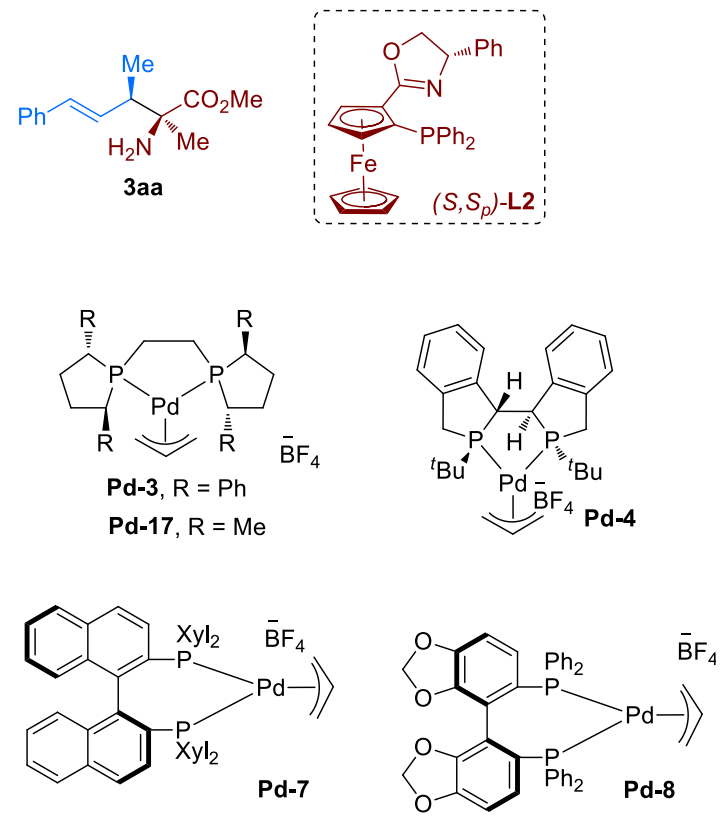

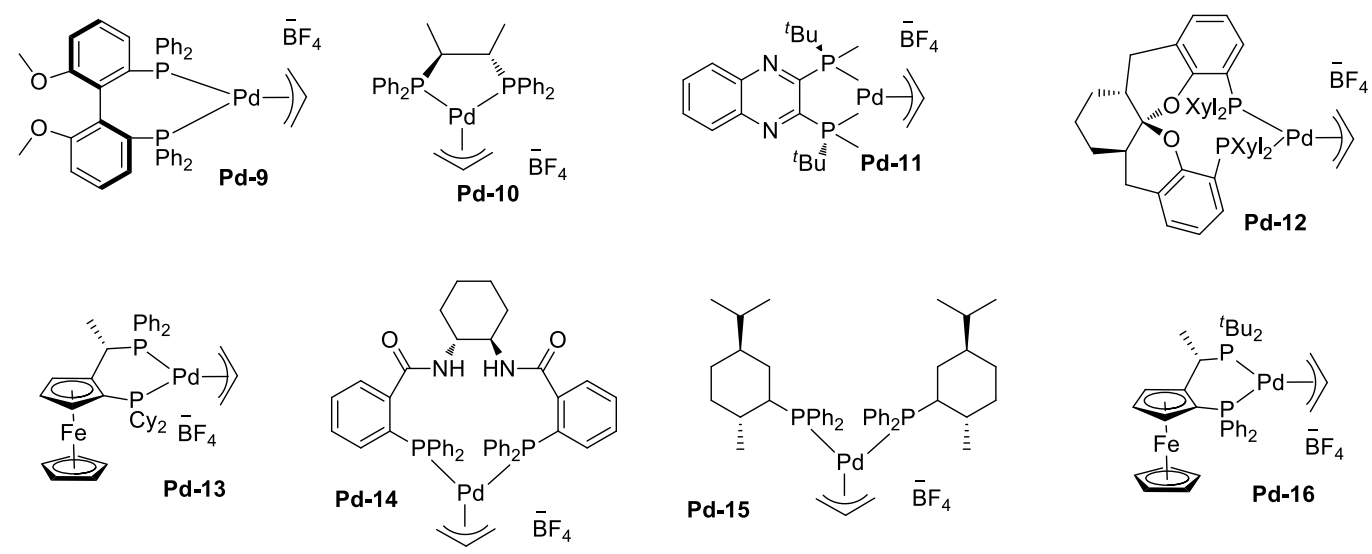

\begin{tabular}{|c|c|c|c|c|c|}
\hline entry & Pd cat. & $\mathrm{Cu}$ ligand $\mathbf{L}$ & yield $(\%)^{b}$ & $\mathrm{dr}^{b}$ & ee $(\%)^{c}$ \\
\hline 1 & Pd-1 & $\left(S, S_{p}\right)-\mathbf{L} \mathbf{2}$ & 75 & $2: 1$ & $95 / 83$ \\
\hline 2 & Pd-2 & $\left(S, S_{p}\right)-\mathbf{L} \mathbf{2}$ & 81 & $4.5: 1$ & $97 / 95$ \\
\hline 3 & Pd-3 & $\left(S, S_{p}\right)-\mathbf{L} \mathbf{2}$ & 62 & $3.5: 1$ & $94 / 94$ \\
\hline 4 & Pd-4 & $\left(S, S_{p}\right)-\mathbf{L} \mathbf{2}$ & 85 & $4.4: 1$ & $99 / 94$ \\
\hline 5 & Pd-5 & $\left(S, S_{p}\right)-\mathbf{L} \mathbf{2}$ & 79 & $7: 1$ & 98/90 \\
\hline 6 & Pd-6 & $\left(S, S_{p}\right)-\mathbf{L} \mathbf{2}$ & $99(88)$ & $>20: 1$ & $>99 /-$ \\
\hline 7 & Pd-6 & $\left(R, R_{p}\right)-\mathbf{L} \mathbf{2}$ & $90(83)$ & $1: 14$ & $-1>99$ \\
\hline 8 & Pd-7 & $\left(S, S_{p}\right)-\mathbf{L} \mathbf{2}$ & 65 & $2.5: 1$ & $93 />97$ \\
\hline 10 & Pd-8 & $\left(S, S_{p}\right)-\mathbf{L} \mathbf{2}$ & 52 & $3.3: 1$ & $95 />87$ \\
\hline 11 & Pd-9 & $\left(S, S_{p}\right)-\mathbf{L} \mathbf{2}$ & 40 & $2.8: 1$ & $93 />80$ \\
\hline 12 & Pd-10 & $\left(S, S_{p}\right)-\mathbf{L} \mathbf{2}$ & 55 & $1.16: 1$ & $85 />97$ \\
\hline 13 & Pd-11 & $\left(S, S_{p}\right)-\mathbf{L} \mathbf{2}$ & 80 & $3.16: 1$ & $99 />94$ \\
\hline 14 & Pd-12 & $\left(S, S_{p}\right)-\mathbf{L} \mathbf{2}$ & 33 & $1.02: 1$ & 90/98 \\
\hline 15 & Pd-13 & $\left(S, S_{p}\right)-\mathbf{L} \mathbf{2}$ & 70 & $1.57: 1$ & 93/97 \\
\hline 16 & Pd-14 & $\left(S, S_{p}\right)-\mathbf{L} \mathbf{2}$ & 20 & $2.3: 1$ & $76 / 98$ \\
\hline 18 & Pd-15 & $\left(S, S_{p}\right)-\mathbf{L} \mathbf{2}$ & 30 & $1.9: 1$ & $83 / 89$ \\
\hline 19 & Pd-16 & $\left(S, S_{p}\right)-\mathbf{L} \mathbf{2}$ & 90 & $>20: 1$ & $>99 /-$ \\
\hline 20 & Pd-17 & $\left(S, S_{p}\right)-\mathbf{L} \mathbf{2}$ & NR & - & -1 \\
\hline
\end{tabular}

${ }^{a}$ Reaction conditions: i) 1a $(0.2 \mathrm{mmol}), \mathbf{2 a}(0.1 \mathrm{mmol}), \mathrm{Pd}$ cat. $(4 \mathrm{~mol} \%), \mathrm{Cu}(\mathrm{MeCN})_{4} \mathrm{PF}_{6}(5 \mathrm{~mol} \%),\left(S, S_{p}\right)-\mathbf{L}$ or $\left(R, R_{p}\right)-\mathbf{L}(5.5 \mathrm{~mol} \%), \mathrm{Et}_{3} \mathrm{~N}(200 \mathrm{~mol} \%)$, THF $(0.5 \mathrm{~mL}), 30{ }^{\circ} \mathrm{C}, 36 \mathrm{~h}$; ii) citric acid $(10 \%, 4 \mathrm{~mL}) .{ }^{b}$ Determined by ${ }^{1} \mathrm{H}$ NMR analysis of the crude product. NR, no reaction. Isolated yields are provided in parentheses. ${ }^{c}$ Determined by HPLC.

\section{General Procedure for Coupling of 1,3-Diene with Aldimine Ester}

Pd catalyst preparation: In an Ar-filled glove box, to a dried Schlenk tube were added $(R, S p)$-Josiphos $(0.6 \mathrm{mmol})$ and $\left[\mathrm{Pd}\left(\eta^{3}-\mathrm{allyl}\right) \mathrm{Cl}\right]_{2}(0.6 \mathrm{mmol})$, dry DCM $(5 \mathrm{~mL})$ was then added and the mixture was stirred at room temperature for $1 \mathrm{~h}$, then $\mathrm{AgBF}_{4}(0.6 \mathrm{mmol})$ 
was added and the mixture was stirred for another $1 \mathrm{~h}$. The mixture was removed from the glove box. The solution was filtered through a pad of celite eluting with $\mathrm{CH}_{2} \mathrm{Cl}_{2}$ (5 $\mathrm{mL} \times 3$ ) and the filtrate was concentrated in vacuo to afford catalyst Pd-6 as a purple solid, which was stored at $-20^{\circ} \mathrm{C}$ under inert atmosphere. The palladium catalyst was used without further purification. The ent-Pd-6 catalyst was prepared using the same procedure but employing $(S, R p)$-Josiphos as the ligand.

Coupling reaction procedure $\mathbf{A}$ : In glove box, $\mathrm{Cu}(\mathrm{MeCN})_{4} \mathrm{PF}_{6}(3.7 \mathrm{mg}, 0.01 \mathrm{mmol}, 5$ mol\%) and chiral ligand $\left(S, S_{p}\right)-\mathbf{L} \mathbf{2}$ or $\left(R, R_{p}\right)-\mathbf{L} \mathbf{2}(5.7 \mathrm{mg}, 0.011 \mathrm{mmol}, 5.5 \mathrm{~mol} \%)$ were dissolved in dry THF $(0.4 \mathrm{M}, 0.5 \mathrm{~mL})$ and stirred at room temperature for $0.5 \mathrm{~h}$. To the solution, substrate aldimine ester 2 (0.2 mmol), Et $3 \mathrm{~N}(0.4 \mathrm{mmol})$, diene $(0.4 \mathrm{mmol})$ and palladium catalyst Pd-6 or ent-Pd-6 $(6.3 \mathrm{mg}, 0.008 \mathrm{mmol}, 4 \mathrm{~mol} \%$ ) were added sequentially. The reaction mixture was stirred at room temperature for $48 \mathrm{~h}$. To the reaction mixture was added citric acid solution $(1 \mathrm{~mL}, 10 \mathrm{wt} . \%)$ and the mixture was stirred for $2 \mathrm{~h}$. The mixture was neutralized with solid $\mathrm{K}_{2} \mathrm{CO}_{3}$ and extracted with EtOAc (10 $\mathrm{mL}$ x 3 ). The combined extracts were dried over $\mathrm{MgSO}_{4}$ and concentrated in vacuo to afford a residue. The crude product was used to determine the diastereoselectivity and regioselectivity by ${ }^{1} \mathrm{H}$ NMR analysis. The residue was then purified by $\mathrm{SiO}_{2}$ column chromatography $(\mathrm{PE} / \mathrm{EA}=5: 1$ to $1: 1)$ to give the desired product.

Reactions in Scheme 2, Table 2 and Table 4 were performed using the above procedure A.

Coupling reaction procedure $\mathbf{B}$ : In glove box, $\mathrm{Cu}(\mathrm{MeCN})_{4} \mathrm{PF}_{6}(5.95 \mathrm{mg}, 0.016 \mathrm{mmol}, 8$ mol\%) and $\left(S, S_{p}\right)-\mathbf{L} 2$ or $\left(R, R_{p}\right)-\mathbf{L} 2(9 \mathrm{mg}, 0.0176 \mathrm{mmol}, 8.8 \mathrm{~mol} \%)$ were dissolved in dry THF $(0.8 \mathrm{M}, 0.25 \mathrm{~mL})$ and stirred at room temperature for $0.5 \mathrm{~h}$. To the solution, substrate aldimine ester $2(0.2 \mathrm{mmol})$, Et $3 \mathrm{~N}(0.4 \mathrm{mmol})$, diene $(0.4 \mathrm{mmol})$ and Pd-6 or ent-Pd-6 ( $9.5 \mathrm{mg}, 0.012 \mathrm{mmol}, 6 \mathrm{~mol} \%$ ) were added sequentially. The reaction mixture was stirred at room temperature for $96 \mathrm{~h}$. To the reaction mixture was added citric acid solution (1 $\mathrm{mL}, 10 \mathrm{wt} . \%$ ) and the mixture was stirred for $2 \mathrm{~h}$. The mixture was neutralized with solid $\mathrm{K}_{2} \mathrm{CO}_{3}$ and extracted with EtOAc $(10 \mathrm{~mL}$ x 3). The combined extracts were dried over $\mathrm{MgSO}_{4}$ and concentrated in vacuo to afford a residue. The crude product was used to determine the diastereoselectivity and regioselectivity by ${ }^{1} \mathrm{H}$ NMR analysis. The residue was then purified by $\mathrm{SiO}_{2}$ column chromatography (PE/EA $=5: 1$ to $1: 1$ ) to give the desired product.

Reactions in Table 3 and Table 5 were performed using the above procedure B.

\section{Characterization of Products}


<smiles>CC(=O)[C@](C)(N)[C@@H](C)/C=C/c1ccccc1</smiles>

$(2 S, 3 R)-3 \mathbf{a a}$

\section{(2S,3R,E)-methyl 2-amino-2,3-dimethyl-5-phenylpent-4-enoate}

Prepared according to General Procedure A using Pd-6 and $(S, S p)-\mathbf{L 2 - C u}$, Purification by flash chromatography $(\mathrm{PE} / \mathrm{EA}=5: 1$ to $1: 1)$ afforded the product as a pale yellow oil, $41 \mathrm{mg}, 88 \%$ yield , >20:1 dr, $>99 \%$ ee, $\mathrm{R}_{\mathrm{f}}=0.4(\mathrm{PE} / \mathrm{EA}, 1: 1)$.

$[\alpha]^{25} \mathrm{D}=51.6\left(\mathrm{c} 1.0, \mathrm{CHCl}_{3}\right)$

${ }^{1} \mathbf{H}$ NMR (400 MHz, CDCl $) \delta 7.37(\mathrm{~d}, J=7.3 \mathrm{~Hz}, 2 \mathrm{H}), 7.30(\mathrm{t}, J=7.5 \mathrm{~Hz}, 2 \mathrm{H}), 7.21(\mathrm{t}, J=7.2 \mathrm{~Hz}$, $1 \mathrm{H}), 6.45(\mathrm{~d}, J=15.8 \mathrm{~Hz}, 1 \mathrm{H}), 6.16(\mathrm{dd}, J=15.8,9.1 \mathrm{~Hz}, 1 \mathrm{H}), 3.71(\mathrm{~s}, 3 \mathrm{H}), 2.72-2.58(\mathrm{~m}, 1 \mathrm{H}), 1.72$ $(\mathrm{s}, 2 \mathrm{H}), 1.32(\mathrm{~s}, 3 \mathrm{H}) .1 .05(\mathrm{~d}, J=6.9 \mathrm{~Hz}, 3 \mathrm{H})$

${ }^{13}$ C NMR (101 MHz, CDCl $) \delta$ 177.87, 137.29, 131.87, 130.55, 128.52, 127.28, 126.24, 60.70, 52.20, 45.91, 24.83, 15.73.

HRMS (ESI) calcd. for $\mathrm{C}_{14} \mathrm{H}_{20} \mathrm{NO}_{2}{ }^{+}(\mathrm{M}+\mathrm{H})^{+}:$:234.1488, Found: 234.1489

the ee value was $>99 \%, \mathrm{t}_{\mathrm{r}}($ minor $)=17.596 \mathrm{~min}, \mathrm{t}_{\mathrm{r}}($ major $)=22.199 \mathrm{~min}($ Chiralcel $\mathrm{IC}, \lambda=220 \mathrm{~nm}$, hexanes $:{ }^{i} \mathrm{PrOH}=98: 2$, flow rate $\left.=1.0 \mathrm{~mL} / \mathrm{min}\right)$.

$\mathrm{mV}$

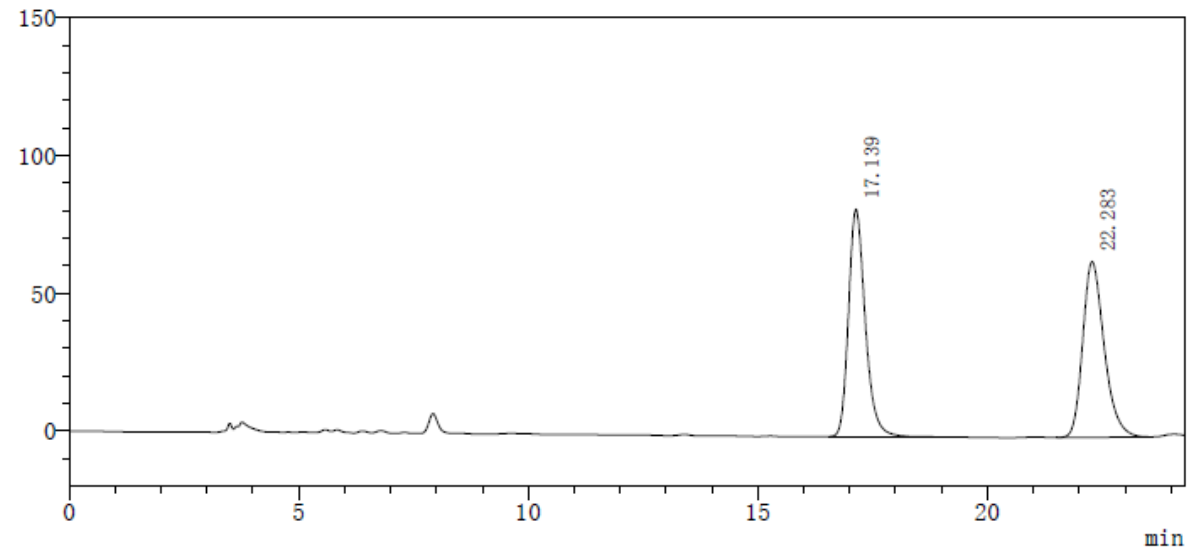

检测器A $220 \mathrm{~nm}$
\begin{tabular}{|c|c|c|c|c|c|}
\hline Peak\# & Time & Area & Height & Height\% & Area\% \\
\hline 1 & 17.139 & 2112051 & 82559 & 56.432 & 50.433 \\
\hline 2 & 22.283 & 2075758 & 63739 & 43.568 & 49.567 \\
\hline 总计 & & 4187809 & 146298 & 100.000 & 100.000 \\
\hline
\end{tabular}

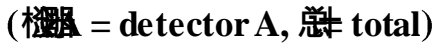


$\mathrm{mV}$

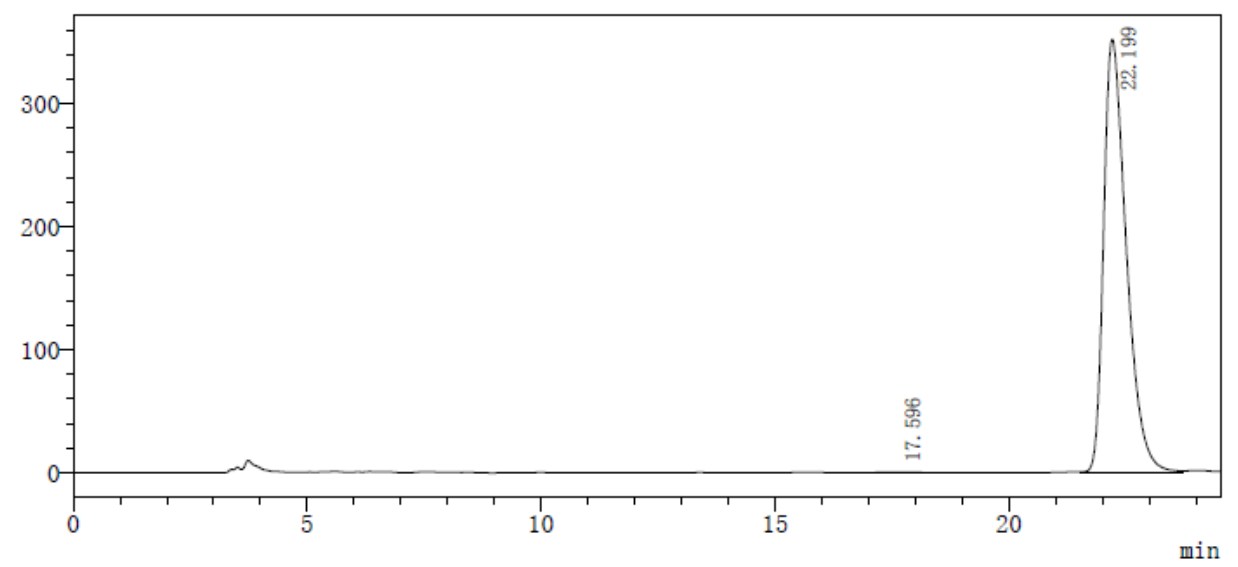

检测器A $220 \mathrm{~nm}$
\begin{tabular}{|c|c|c|c|c|c|}
\hline Peak\# & Time & Area & Height & Height\% & Area\% \\
\hline 1 & 17.596 & 22898 & 318 & 0.090 & 0.192 \\
\hline 2 & 22.199 & 11894279 & 352277 & 99.910 & 99.808 \\
\hline 总计 & & 11917177 & 352596 & 100.000 & 100.000 \\
\hline
\end{tabular}<smiles>CC(=O)O[14C](C)(N)/C=C/c1ccccc1</smiles>

(2R,3S)-3aa

\section{(2R,3S,E)-methyl 2-amino-2,3-dimethyl-5-phenylpent-4-enoate}

Prepared according to General Procedure A using ent-Pd-6 and $(R, R p)-\mathbf{L 2 - C u}$, Purification by flash chromatography (PE/EA $=5: 1$ to 1:1) afforded the product as a pale yellow oil, $39.1 \mathrm{mg}, 84 \%$ yield , >20:1 dr, $>99 \%$ ee, $\mathrm{R}_{\mathrm{f}}=0.4(\mathrm{PE} / \mathrm{EA}, 1: 1)$.

$[\alpha]^{25} \mathrm{D}=-52.8\left(\mathrm{c} 0.2, \mathrm{CHCl}_{3}\right)$

The ${ }^{1} \mathrm{H}$ NMR and ${ }^{13} \mathrm{C}$ NMR data is the same as $(2 S, 3 R, E)-3 \mathbf{a a}$

the ee value $\mathrm{was}>99 \%, \mathrm{t}_{\mathrm{r}}$ (major) $=17.490 \mathrm{~min}, \mathrm{t}_{\mathrm{r}}($ minor $)=22.479 \mathrm{~min}$ (Chiralcel $\mathrm{IC}, \lambda=220 \mathrm{~nm}$, hexanes $:{ }^{i} \mathrm{PrOH}=98: 2$, flow rate $=1.0 \mathrm{~mL} / \mathrm{min}$ ) 
$\mathrm{mV}$

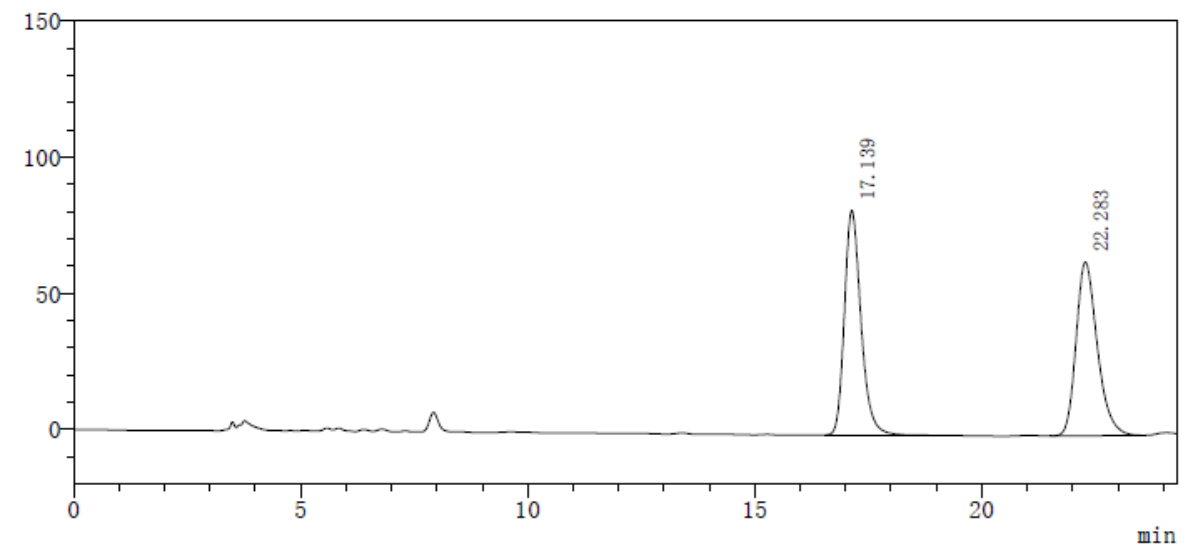

检测器A $220 \mathrm{~nm}$
\begin{tabular}{|c|c|c|c|c|c|}
\hline Peak\# & Time & Area & Height & Height\% & Area\% \\
\hline 1 & 17.139 & 2112051 & 82559 & 56.432 & 50.433 \\
\hline 2 & 22.283 & 2075758 & 63739 & 43.568 & 49.567 \\
\hline 总计 & & 4187809 & 146298 & 100.000 & 100.000 \\
\hline
\end{tabular}

$\mathrm{mV}$

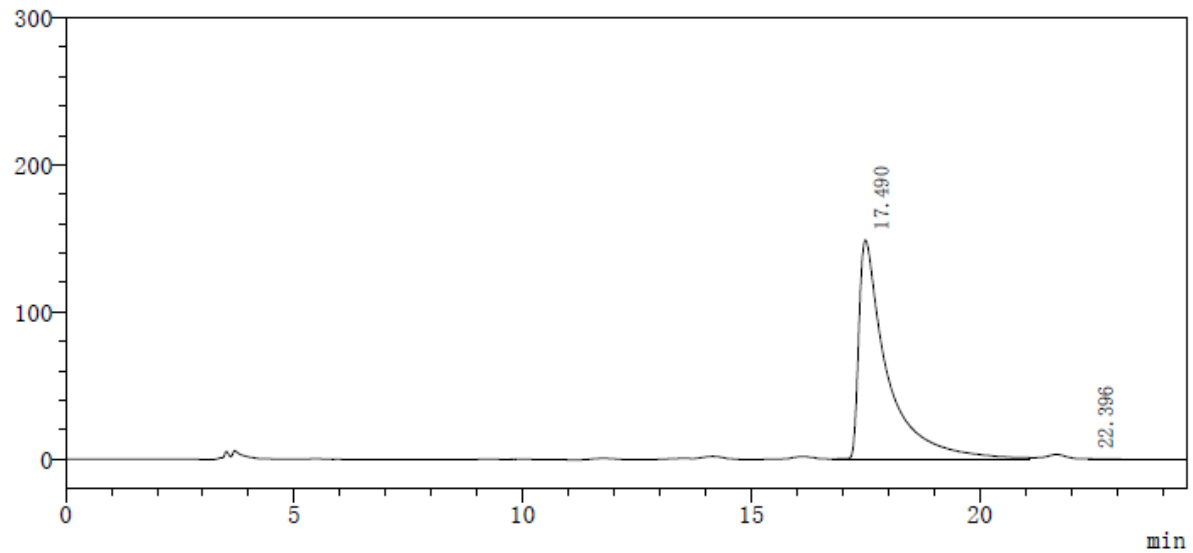

检测器A $220 \mathrm{~nm}$

\begin{tabular}{|c|c|c|c|c|c|}
\hline Peak\# & Time & Area & Height & Height\% & Area\% \\
\hline 1 & 17.490 & 6497689 & 149305 & 99.811 & 99.887 \\
\hline 2 & 22.396 & 7338 & 283 & 0.189 & 0.113 \\
\hline 总计 & & 6505027 & 149588 & 100.000 & 100.000 \\
\hline
\end{tabular}<smiles>COC(=O)[C@](C)(N)[C@@H](C)/C=C/c1ccccc1</smiles>

$(2 R, 3 R)-3$ aa

\section{(2R,3R,E)-methyl 2-amino-2,3-dimethyl-5-phenylpent-4-enoate}

Prepared according to General Procedure A using Pd-6 and $(R, R p)-\mathbf{L 2}-\mathbf{C u}$, Purification by flash chromatography (PE/EA = 5:1 to 1:1) afforded the product as a pale yellow oil, $38.7 \mathrm{mg}, 83 \%$ yield, 14.0:1 dr, >99\% ee, $\mathrm{R}_{\mathrm{f}}=0.35$ (PE/EA, 1:1).

$[\alpha]^{25} \mathrm{D}=33.7\left(\mathrm{c} 0.4, \mathrm{CHCl}_{3}\right)$

${ }^{1}$ H NMR (400 MHz, CDCl $) \delta 7.39-7.27(\mathrm{~m}, 4 \mathrm{H}), 7.21(\mathrm{t}, J=7.0 \mathrm{~Hz}, 1 \mathrm{H}), 6.41(\mathrm{~d}, J=15.8 \mathrm{~Hz}$, 
$1 \mathrm{H}), 6.12(\mathrm{dd}, J=15.8,8.5 \mathrm{~Hz}, 1 \mathrm{H}), 3.71(\mathrm{~s}, 3 \mathrm{H}), 2.79-2.55(\mathrm{~m}, 1 \mathrm{H}), 1.75(\mathrm{~s}, 2 \mathrm{H}), 1.32(\mathrm{~s}, 3 \mathrm{H}), 1.12$ $(\mathrm{d}, J=6.8 \mathrm{~Hz}, 3 \mathrm{H})$.

${ }^{13}$ C NMR (101 MHz, CDCl $) \delta$ 177.39, 137.26, 131.51, 130.81, 128.52, 127.33, 126.24, 61.02, 52.14, $45.64,23.47,14.38$

HRMS (ESI) calcd. for $\mathrm{C}_{14} \mathrm{H}_{20} \mathrm{NO}_{2}{ }^{+}(\mathrm{M}+\mathrm{H})^{+}: 234.1488$, Found: 234.1494

the ee value $\mathrm{was}>99 \%, \mathrm{t}_{\mathrm{r}}($ major $)=23.681 \mathrm{~min}, \mathrm{t}_{\mathrm{r}}($ minor $)=28.180 \mathrm{~min}$ (Chiralcel $\mathrm{IC}, \lambda=220 \mathrm{~nm}$, hexanes $:{ }^{i} \mathrm{PrOH}=98: 2$, flow rate $\left.=1.0 \mathrm{~mL} / \mathrm{min}\right)$.

$\mathrm{mV}$

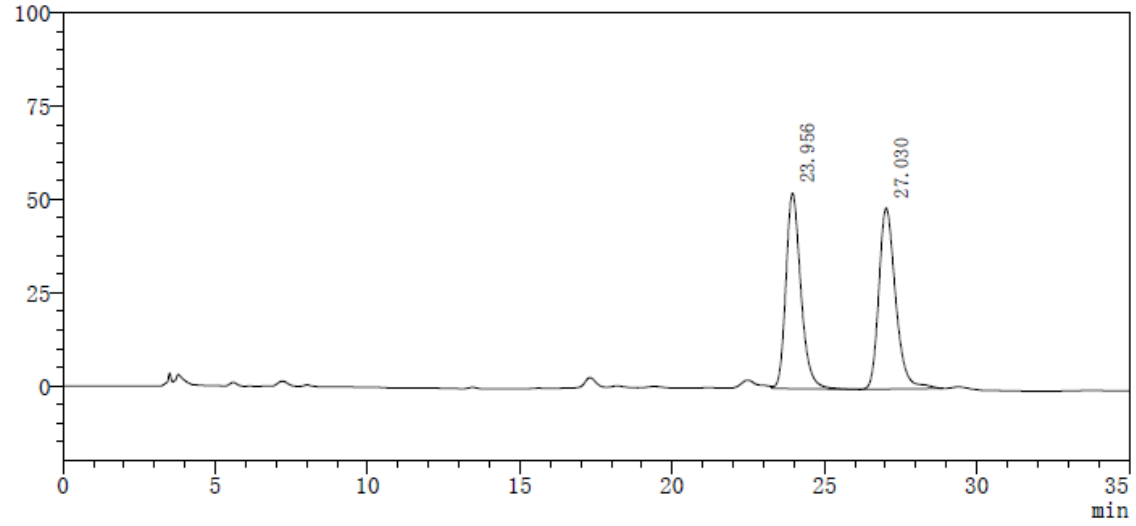

检测器A $220 \mathrm{~nm}$
\begin{tabular}{|c|c|c|c|c|c|}
\hline Peak\# & Time & Area & Height & Height\% & Area\% \\
\hline 1 & 23.956 & 1784960 & 52364 & 51.915 & 49.023 \\
\hline 2 & 27.030 & 1856098 & 48501 & 48.085 & 50.977 \\
\hline 总计 & & 3641059 & 100865 & 100.000 & 100.000 \\
\hline
\end{tabular}

$\mathrm{mV}$

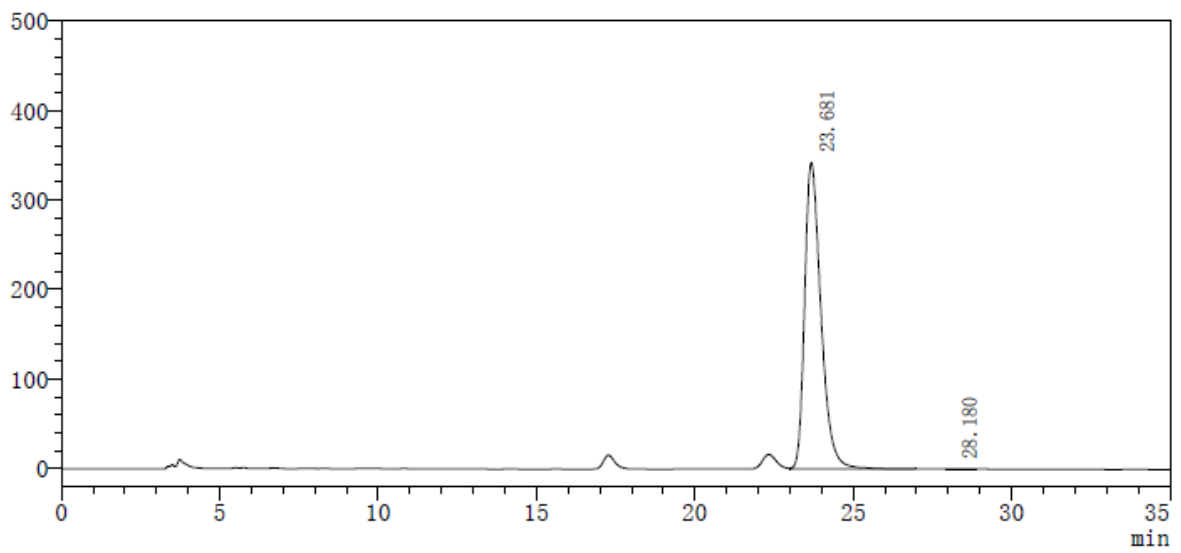

\begin{tabular}{l} 
检测器A 220nm \\
\hline Peak\#
\end{tabular}<smiles>CC(=O)C(N)(N)[C@@H](C)/C=C/c1ccccc1</smiles>

$(2 S, 3 S)-3 \mathbf{a a}$ 


\section{(2S,3S,E)-methyl 2-amino-2,3-dimethyl-5-phenylpent-4-enoate}

Prepared according to General Procedure A using ent-Pd-6 and $(S, S p)$-L2-Cu, Purification by flash chromatography (PE/EA = 5:1 to 1:1) afforded the product as a pale yellow oil, $38 \mathrm{mg}, 82 \%$ yield, 13.6:1 dr, $>99 \%$ ee, $R_{\mathrm{f}}=0.35(\mathrm{PE} / \mathrm{EA}, 1: 1)$.

$[\alpha]^{25} \mathrm{D}=-33.0\left(\mathrm{c} 0.5, \mathrm{CHCl}_{3}\right)$

The ${ }^{1} \mathrm{H}$ NMR and ${ }^{13} \mathrm{C}$ NMR data is the same as $(2 R, 3 R, E)$-3aa

the ee value was $>99 \%, \mathrm{t}_{\mathrm{r}}($ minor $)=24.088 \mathrm{~min}, \mathrm{t}_{\mathrm{r}}($ major $)=27.289 \min ($ Chiralcel $\mathrm{IC}, \lambda=220 \mathrm{~nm}$, hexanes $:{ }^{i} \mathrm{PrOH}=98: 2$, flow rate $=1.0 \mathrm{~mL} / \mathrm{min}$ ).

$\mathrm{mV}$

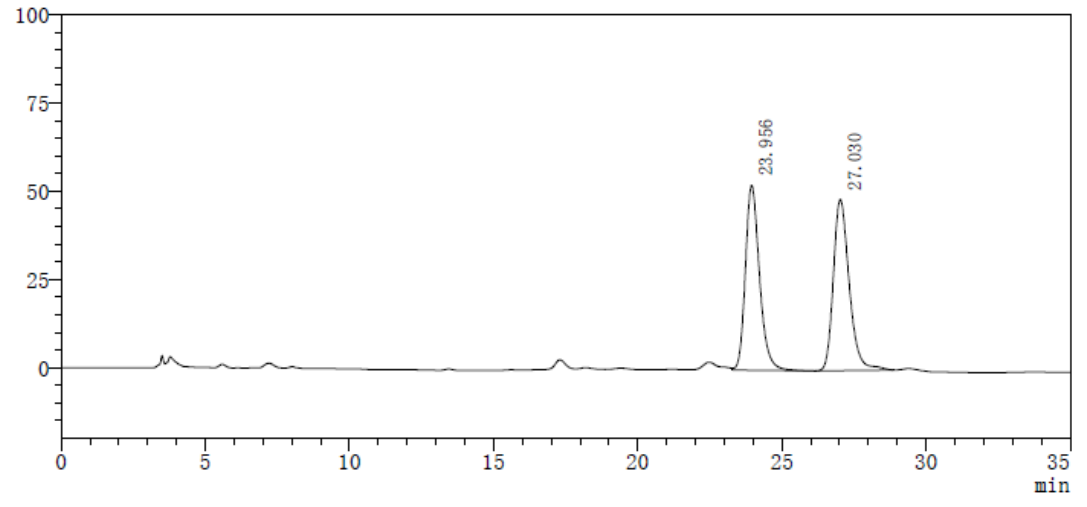

检测器A $220 \mathrm{~nm}$
\begin{tabular}{|c|c|c|c|c|c|}
\hline Peak\# & Time & Area & Height & Height\% & Area\% \\
\hline 1 & 23.956 & 1784960 & 52364 & 51.915 & 49.023 \\
\hline 2 & 27.030 & 1856098 & 48501 & 48.085 & 50.977 \\
\hline 总计 & & 3641059 & 100865 & 100.000 & 100.000 \\
\hline
\end{tabular}

$\mathrm{mV}$

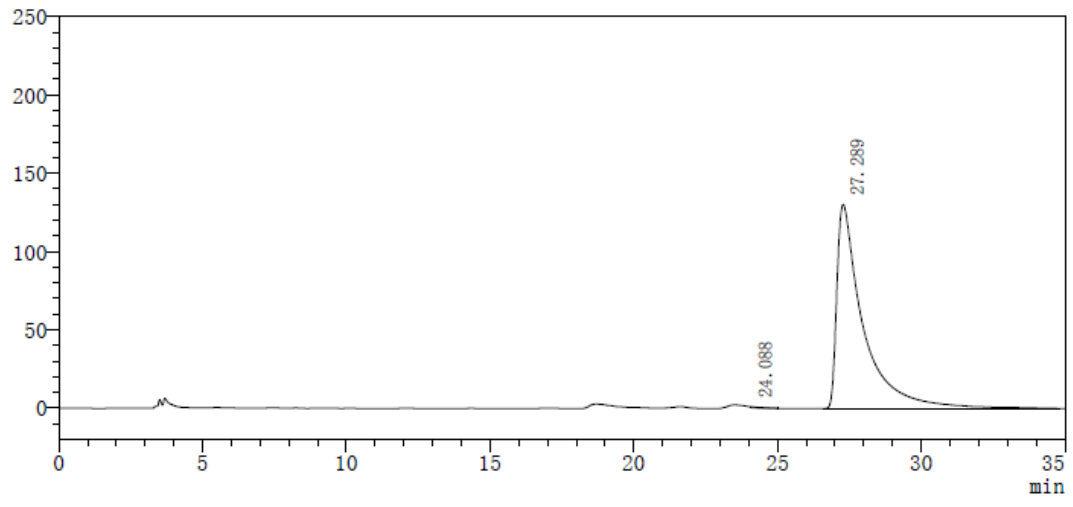

检测器A $220 \mathrm{~nm}$
\begin{tabular}{|c|c|c|c|c|c|}
\hline Peak\# & Time & Area & Height & Height\% & Area\% \\
\hline 1 & 24.088 & 15567 & 746 & 0.570 & 0.188 \\
\hline 2 & 27.289 & 8285916 & 130098 & 99.430 & 99.812 \\
\hline 总计 & & 8301483 & 130844 & 100.000 & 100.000 \\
\hline
\end{tabular}<smiles>[M]C(C=Cc1ccc(C)cc1)C(C)(N)C(C)=O</smiles>

$(2 S, 3 R)-3 \mathbf{b a}$

(2S,3R,E)-methyl 2-amino-2,3-dimethyl-5-(p-tolyl)pent-4-enoate 
Prepared according to General Procedure A using Pd-6 and $(S, S p)$-L2-Cu, Purification by flash chromatography $(\mathrm{PE} / \mathrm{EA}=5: 1$ to $1: 1)$ afforded the product as a pale yellow oil, $34 \mathrm{mg}, 68 \%$ yield, $>20: 1 \mathrm{dr},>99 \%$ ee, $\mathrm{R}_{\mathrm{f}}=0.4(\mathrm{PE} / \mathrm{EA}, 1: 1)$.

$[\alpha]^{25} \mathrm{D}=67.6\left(\mathrm{c} 0.5, \mathrm{CHCl}_{3}\right)$

${ }^{1}$ H NMR (400 MHz, CDCl $) \delta 7.27(\mathrm{~d}, J=6.5 \mathrm{~Hz}, 2 \mathrm{H}), 7.11(\mathrm{~d}, J=7.8 \mathrm{~Hz}, 2 \mathrm{H}), 6.41(\mathrm{~d}, J=15.8 \mathrm{~Hz}$, $1 \mathrm{H}), 6.10(\mathrm{dd}, J=15.8,9.1 \mathrm{~Hz}, 1 \mathrm{H}), 3.73(\mathrm{~s}, 3 \mathrm{H}), 2.73-2.55(\mathrm{~m}, 1 \mathrm{H}), 2.33(\mathrm{~s}, 3 \mathrm{H}), 1.65(\mathrm{~s}, 2 \mathrm{H}), 1.31$ $(\mathrm{s}, 3 \mathrm{H}), 1.04(\mathrm{~d}, J=6.8 \mathrm{~Hz}, 3 \mathrm{H})$.

${ }^{13}$ C NMR (101 MHz, CDCl $) \delta$ 177.80, 137.05, 134.52, 131.73, 129.45, 129.21, 126.13, 60.74, 52.16, 45.91, 24.81, 21.16, 15.74.

HRMS (ESI) calcd. for $\mathrm{C}_{15} \mathrm{H}_{22} \mathrm{NO}_{2}{ }^{+}(\mathrm{M}+\mathrm{H})^{+}:$:248.1651, Found: 248.1646

the ee value was $>99 \%, \mathrm{t}_{\mathrm{r}}($ major $)=17.649 \mathrm{~min}, \mathrm{t}_{\mathrm{r}}($ minor $)=24.738 \mathrm{~min}($ Chiralcel $\mathrm{IC}, \lambda=220 \mathrm{~nm}$, hexanes $:{ }^{i} \mathrm{PrOH}=98: 2$, flow rate $\left.=1.0 \mathrm{~mL} / \mathrm{min}\right)$.

$\mathrm{mV}$

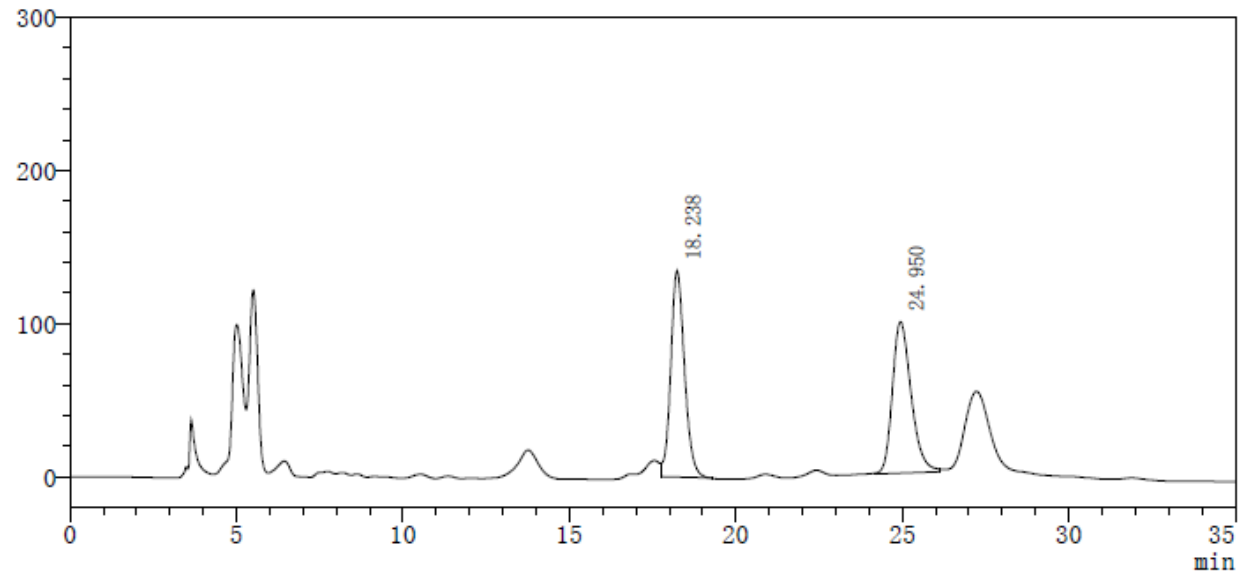

检测器A 220 nm
\begin{tabular}{|c|c|c|c|c|c|}
\hline Peak\# & Time & Area & Height & Height\% & Area\% \\
\hline 1 & 18.238 & 3884400 & 134657 & 57.681 & 50.093 \\
\hline 2 & 24.950 & 3869945 & 98795 & 42.319 & 49.907 \\
\hline 总计 & & 7754346 & 233452 & 100.000 & 100.000 \\
\hline
\end{tabular}


$\mathrm{mV}$

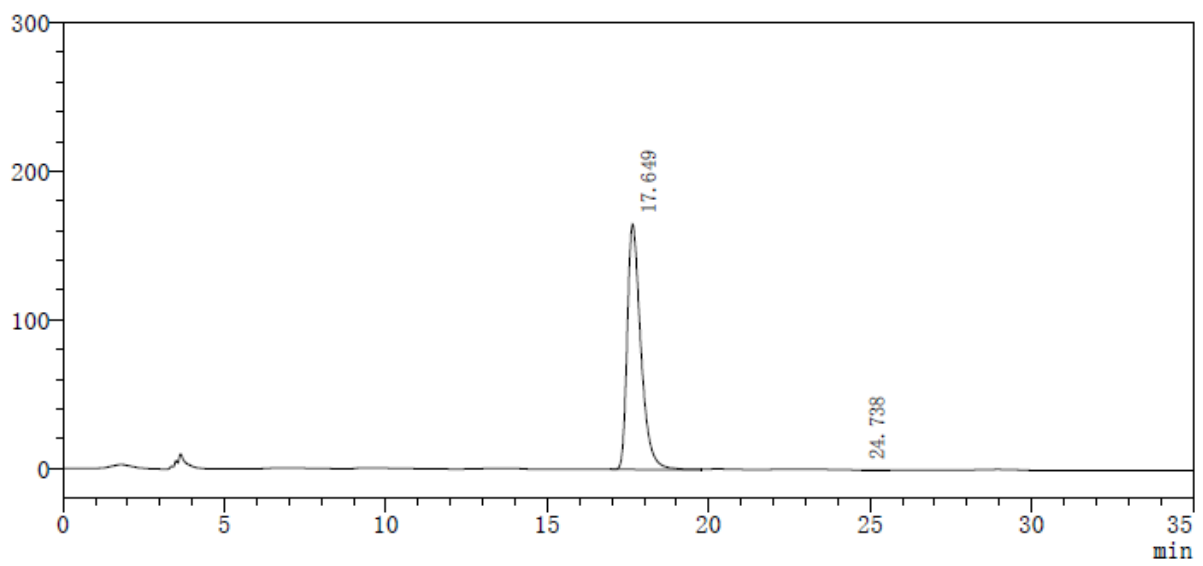

检测器A $220 \mathrm{~nm}$
\begin{tabular}{|c|c|c|c|c|c|}
\hline Peak\# & Time & Area & Height & Height\% & Area\% \\
\hline 1 & 17.649 & 4841042 & 165084 & 99.879 & 99.858 \\
\hline 2 & 24.738 & 6875 & 201 & 0.121 & 0.142 \\
\hline 总计 & & 4847917 & 165285 & 100.000 & 100.000 \\
\hline
\end{tabular}<smiles>CC(=O)C(C)(N)C([Mg])C=Cc1cccc(C)c1</smiles>

$(2 S, 3 R)-3 \mathbf{c a}$

\section{(2S,3R,E)-methyl 2-amino-2,3-dimethyl-5-(m-tolyl)pent-4-enoate}

Prepared according to General Procedure A using Pd-6 and $(S, S p)$-L2-Cu, Purification by flash chromatography $(\mathrm{PE} / \mathrm{EA}=5: 1$ to $1: 1)$ afforded the product as a pale yellow oil, $41 \mathrm{mg}, 83 \%$ yield, $>20: 1 \mathrm{dr},>99 \%$ ee, $\mathrm{R}_{\mathrm{f}}=0.4(\mathrm{PE} / \mathrm{EA}, 1: 1)$.

$[\alpha]^{25} \mathrm{D}=58.9\left(\mathrm{c} 1.0, \mathrm{CHCl}_{3}\right)$

${ }^{1}$ H NMR (400 MHz, CDCl $) \delta 7.23-7.11(\mathrm{~m}, 3 \mathrm{H}), 7.03(\mathrm{~d}, J=6.6 \mathrm{~Hz}, 1 \mathrm{H}), 6.42(\mathrm{~d}, J=15.8 \mathrm{~Hz}, 1 \mathrm{H})$, $6.15(\mathrm{dd}, J=15.8,9.1 \mathrm{~Hz}, 1 \mathrm{H}), 3.73(\mathrm{~s}, 3 \mathrm{H}), 2.74-2.58(\mathrm{~m}, 1 \mathrm{H}), 2.33(\mathrm{~s}, 3 \mathrm{H}), 1.67(\mathrm{~s}, 2 \mathrm{H}), 1.31$ (s, $3 \mathrm{H}), 1.04(\mathrm{~d}, J=6.8 \mathrm{~Hz}, 3 \mathrm{H})$.

${ }^{13}$ C NMR (101 MHz, CDCl 3$) \delta 177.83,138.07,137.22,131.95,130.31,128.43,128.08,126.84$, $123.49,60.71,52.19,45.89,24.88,21.40,15.77$.

HRMS (ESI) calcd. for $\mathrm{C}_{15} \mathrm{H}_{22} \mathrm{NO}_{2}{ }^{+}(\mathrm{M}+\mathrm{H})^{+}:$248.1651, Found: 248.1649

the ee value $\mathrm{was}>99 \%, \mathrm{t}_{\mathrm{r}}($ minor $)=16.606 \mathrm{~min}, \mathrm{t}_{\mathrm{r}}$ (major) $=21.935 \mathrm{~min}$ (Chiralcel $\mathrm{IC}, \lambda=220 \mathrm{~nm}$, hexanes $:{ }^{i} \mathrm{PrOH}=98: 2$, flow rate $=1.0 \mathrm{~mL} / \mathrm{min}$ ). 
$\mathrm{mV}$

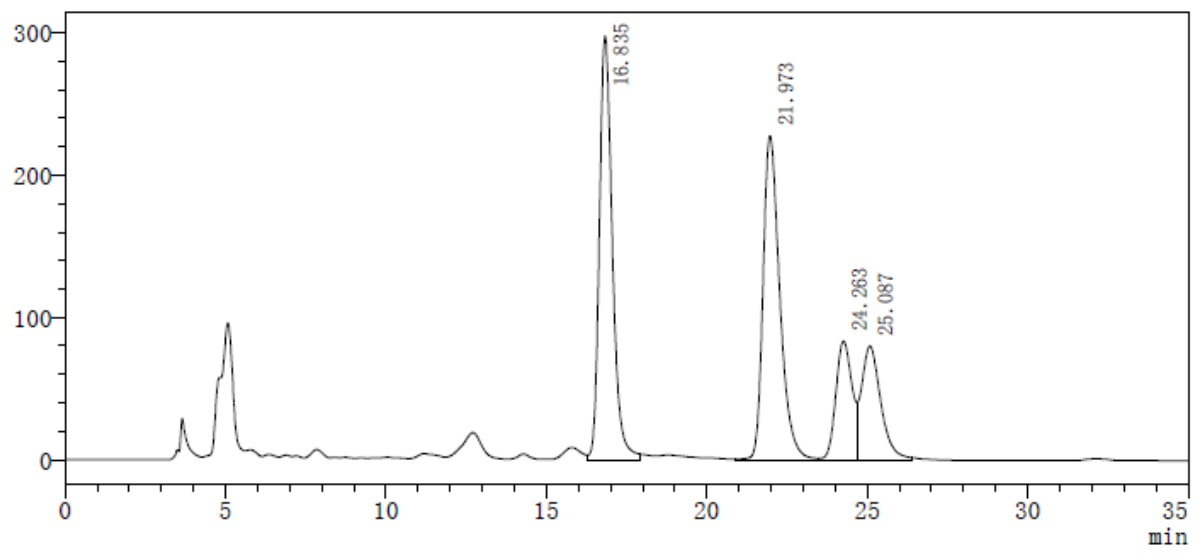

检测器A 220nm
\begin{tabular}{|c|c|c|c|c|c|}
\hline Peak\# & Time & Area & Height & Height\% & Area\% \\
\hline 1 & 16.835 & 8391396 & 298231 & 43.149 & 36.350 \\
\hline 2 & 21.973 & 8311123 & 228440 & 33.051 & 36.003 \\
\hline 3 & 24.263 & 2959274 & 84051 & 12.161 & 12.819 \\
\hline 4 & 25.087 & 3422938 & 80452 & 11.640 & 14.828 \\
\hline 总计 & & 23084731 & 691173 & 100.000 & 100.000 \\
\hline
\end{tabular}

$\mathrm{mV}$

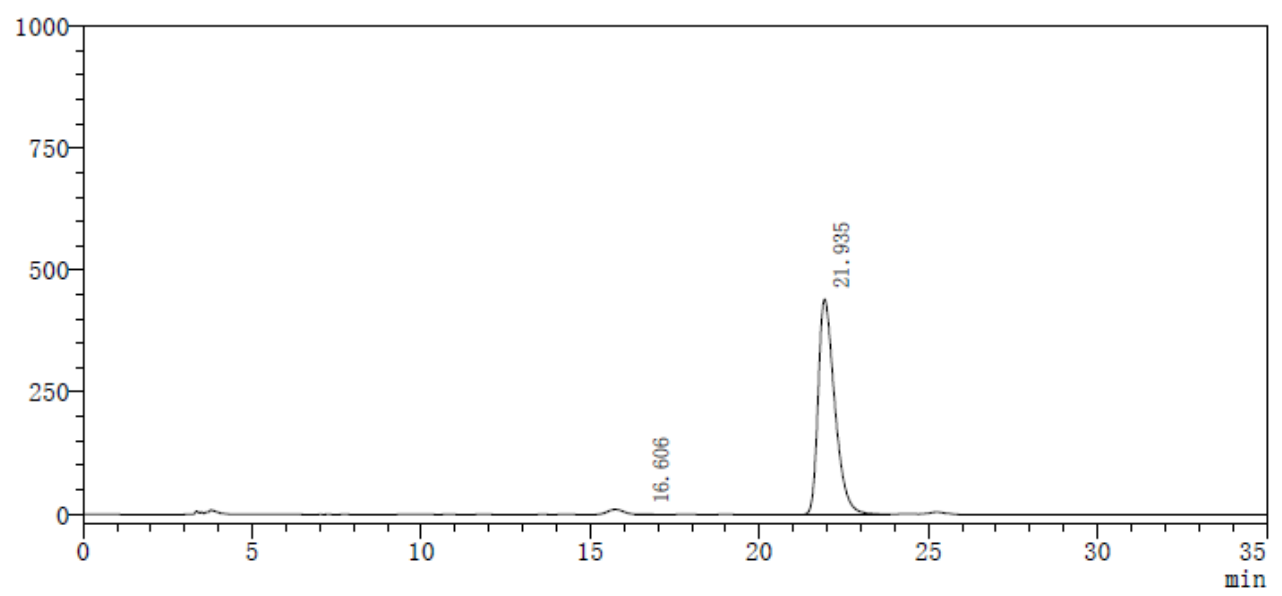

检测器A 220nm
\begin{tabular}{|c|c|c|c|c|c|}
\hline Peak\# & Time & Area & Height & Height\% & Area\% \\
\hline 1 & 16.606 & 28916 & 777 & 0.176 & 0.191 \\
\hline 2 & 21.935 & 15100766 & 441677 & 99.824 & 99.809 \\
\hline 总计 & & 15129682 & 442453 & 100.000 & 100.000 \\
\hline
\end{tabular}<smiles>COC(=O)[C@](C)(N)[C@@H](C)/C=C/c1cccc(C)c1</smiles>

$(2 R, 3 R)-3 \mathbf{c a}$

(2R,3R,E)-methyl 2-amino-2,3-dimethyl-5-(m-tolyl)pent-4-enoate

Prepared according to General Procedure A using using Pd-6 and $(R, R p)$-L2-Cu, Purification by flash 
chromatography (PE/EA = 5:1 to 1:1) afforded the product as a pale yellow oil, $35 \mathrm{mg}, 77 \%$ yield, $10: 1 \mathrm{dr}, 99 \%$ ee, $\mathrm{R}_{\mathrm{f}}=0.35$ (PE/EA, $\left.1: 1\right)$.

$[\alpha]^{25} \mathrm{D}=52.6\left(\mathrm{c} 0.5, \mathrm{CHCl}_{3}\right)$

${ }^{1}$ H NMR (400 MHz, CDCl $) \delta 7.21-7.11(\mathrm{~m}, 3 \mathrm{H}), 7.03(\mathrm{~d}, J=7.3 \mathrm{~Hz}, 1 \mathrm{H}), 6.38(\mathrm{~d}, J=15.8 \mathrm{~Hz}, 1 \mathrm{H})$, $6.10(\mathrm{dd}, J=15.8,8.5 \mathrm{~Hz}, 1 \mathrm{H}), 3.73(\mathrm{~s}, 3 \mathrm{H}), 2.76-2.61(\mathrm{~m}, 1 \mathrm{H}), 2.33(\mathrm{~s}, 3 \mathrm{H}), 1.64(\mathrm{~s}, 2 \mathrm{H}), 1.32(\mathrm{~s}$, $3 \mathrm{H}), 1.11(\mathrm{~d}, J=6.9 \mathrm{~Hz}, 3 \mathrm{H})$.

${ }^{13}$ C NMR (101 MHz, CDCl $) \delta 177.47,138.07,137.20,131.58,130.59,128.43,128.12,126.89$, $123.44,61.01,52.12,45.66,23.48,21.39,14.41$.

HRMS (ESI) calcd. for $\mathrm{C}_{15} \mathrm{H}_{22} \mathrm{NO}_{2}{ }^{+}(\mathrm{M}+\mathrm{H})^{+}: 248.1651$, Found: 248.1646

the ee value $\mathrm{was} 99 \%, \mathrm{t}_{\mathrm{r}}($ major $)=24.000 \mathrm{~min}, \mathrm{t}_{\mathrm{r}}($ minor $)=25.454 \min ($ Chiralcel $\mathrm{IC}, \lambda=220 \mathrm{~nm}$, hexanes $:{ }^{i} \mathrm{PrOH}=98: 2$, flow rate $\left.=1.0 \mathrm{~mL} / \mathrm{min}\right)$.

$\mathrm{mV}$
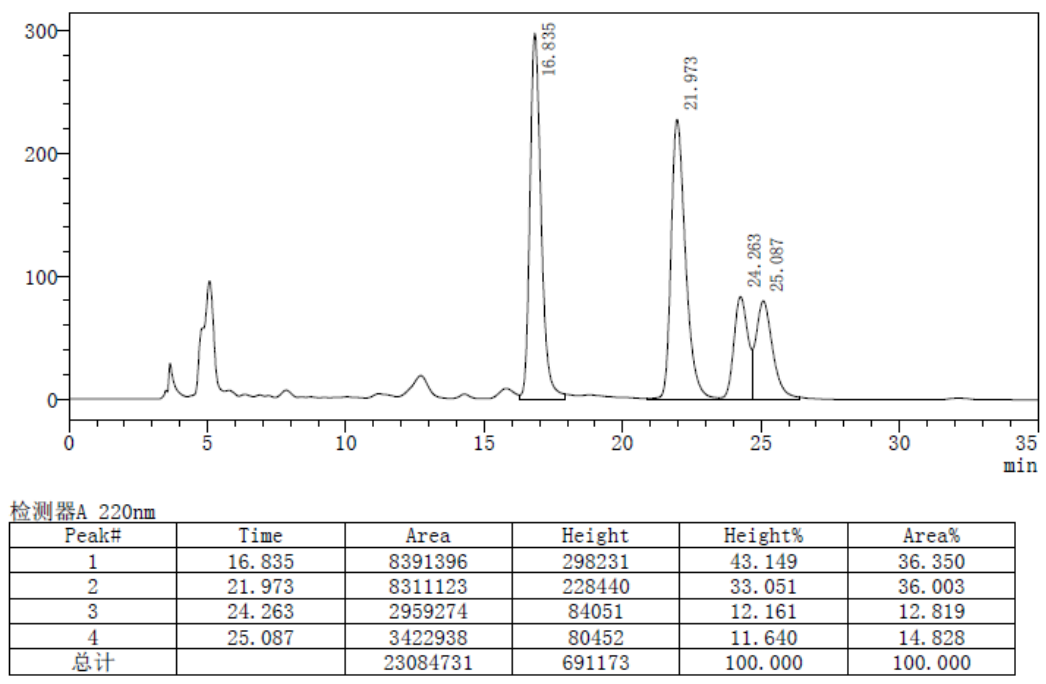

$\mathrm{mV}$

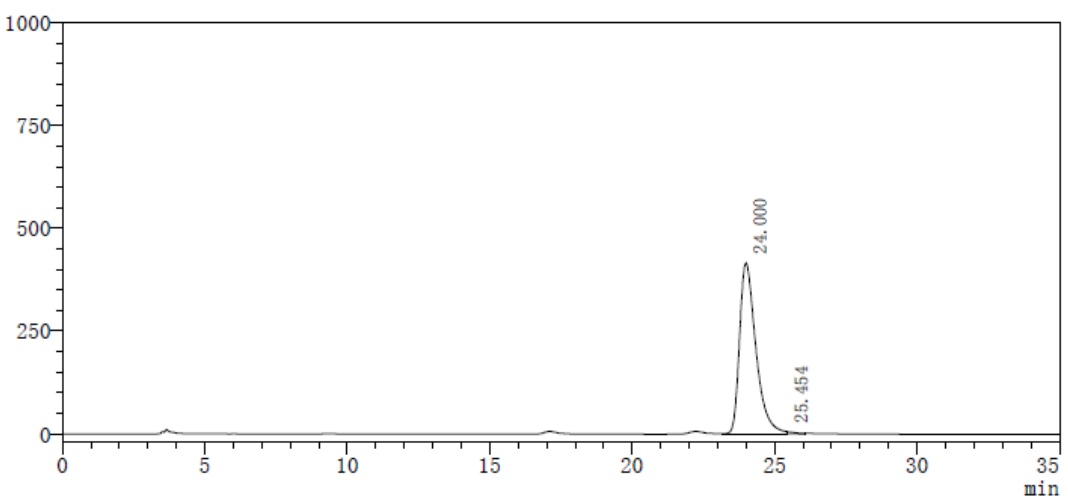

检测器A $220 \mathrm{~nm}$
\begin{tabular}{|c|c|c|c|c|c|}
\hline Peak\# & Time & Area & Height & Height $\%$ & Area\% \\
\hline 1 & 24.000 & 16511647 & 414903 & 98.838 & 99.405 \\
\hline 2 & 25.454 & 98796 & 4876 & 1.162 & 0.595 \\
\hline 总计 & & 16610443 & 419779 & 100.000 & 100.000 \\
\hline
\end{tabular}


<smiles>CC(=O)C(C)(N)C(C)C=Cc1ccccc1F</smiles>

$(2 S, 3 R)-3 \mathbf{d a}$

(2S,3R,E)-methyl 2-amino-5-(2-fluorophenyl)-2,3-dimethylpent-4-enoate

Prepared according to General Procedure A using Pd-6 and $(S, S p)-\mathbf{L 2}-\mathbf{C u}$, Purification by flash chromatography (PE/EA $=5: 1$ to 1:1) afforded the product as a pale yellow oil, $41 \mathrm{mg}, 83 \%$ yield, $>20: 1 \mathrm{dr},>99 \%$ ee, $\mathrm{R}_{\mathrm{f}}=0.4(\mathrm{PE} / \mathrm{EA}, 1: 1)$.

$[\alpha]^{25}{ }_{\mathrm{D}}=59.1\left(\mathrm{c} 1.0, \mathrm{CHCl}_{3}\right)$

${ }^{1}$ H NMR (400 MHz, CDCl $) \delta 7.46(\mathrm{t}, J=7.6 \mathrm{~Hz}, 1 \mathrm{H}), 7.18(\mathrm{dd}, J=13.4,6.7 \mathrm{~Hz}, 1 \mathrm{H}), 7.07(\mathrm{t}, J=$ $7.5 \mathrm{~Hz}, 1 \mathrm{H}), 7.05-6.98(\mathrm{~m}, 1 \mathrm{H}), 6.61(\mathrm{~d}, J=16.0 \mathrm{~Hz}, 1 \mathrm{H}), 6.24(\mathrm{dd}, J=16.0,9.1 \mathrm{~Hz}, 1 \mathrm{H}), 3.73(\mathrm{~s}$, $3 \mathrm{H}), 2.73-2.63(\mathrm{~m}, 1 \mathrm{H}), 1.68(\mathrm{~s}, 2 \mathrm{H}), 1.32(\mathrm{~s}, 3 \mathrm{H}), 1.06(\mathrm{~d}, J=6.8 \mathrm{~Hz}, 3 \mathrm{H})$.

${ }^{13}$ C NMR (101 MHz, CDCl $) \delta 177.75,160.00(\mathrm{~d}, J=248.6 \mathrm{~Hz}), 133.26(\mathrm{~d}, J=4.3 \mathrm{~Hz}), 128.49$ (d, $J$ $=8.3 \mathrm{~Hz}), 127.18(\mathrm{~d}, J=3.8 \mathrm{~Hz}), 125.04(\mathrm{~d}, J=12.3 \mathrm{~Hz}), 124.04(\mathrm{~d}, J=7.9 \mathrm{~Hz}), 124.04,115.63(\mathrm{~d}, J$ $=22.1 \mathrm{~Hz}), 60.65,52.20,46.19,24.74,15.62$

${ }^{19}$ F NMR (376 MHz, $\left.\mathbf{C D C l}_{3}\right) \delta-123.66$.

HRMS (ESI) calcd. for $\mathrm{C}_{14} \mathrm{H}_{19} \mathrm{FNO}_{2}{ }^{+}(\mathrm{M}+\mathrm{H})^{+}: 252.1400$, Found: 252.1397

the ee value was $>99 \%, \mathrm{t}_{\mathrm{r}}($ minor $)=15.538 \mathrm{~min}, \mathrm{t}_{\mathrm{r}}($ major $)=17.210 \min$ (Chiralcel $\mathrm{IC}, \lambda=220 \mathrm{~nm}$, hexanes $:{ }^{i} \mathrm{PrOH}=98: 2$, flow rate $\left.=1.0 \mathrm{~mL} / \mathrm{min}\right)$.

$\mathrm{mV}$

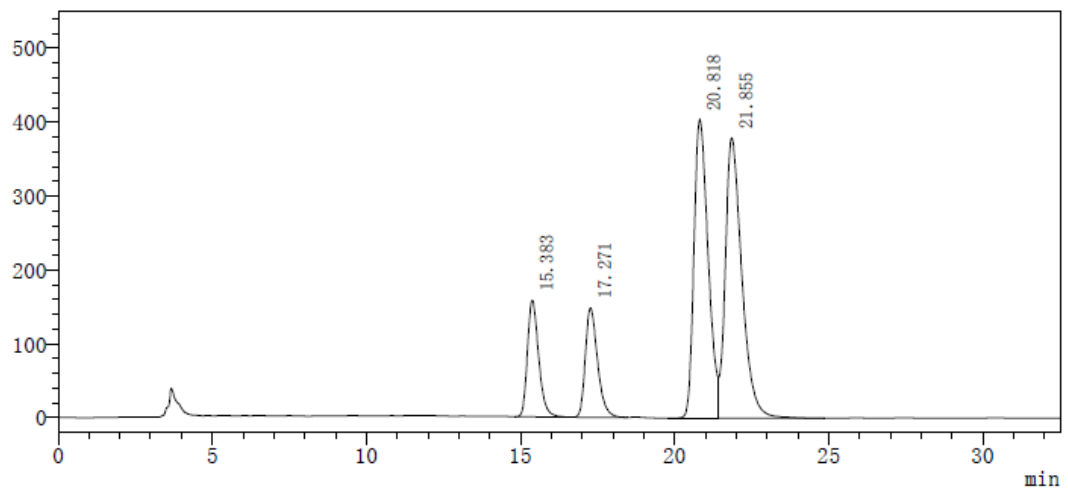

检测器A $220 \mathrm{~nm}$
\begin{tabular}{|c|c|c|c|c|c|}
\hline Peak\# & Time & Area & Height & Height\% & Area\% \\
\hline 1 & 15.383 & 4007779 & 157761 & 14.479 & 11.381 \\
\hline 2 & 17.271 & 4075638 & 148191 & 13.600 & 11.574 \\
\hline 3 & 20.818 & 12933755 & 404280 & 37.103 & 36.729 \\
\hline 4 & 21.855 & 14196467 & 379390 & 34.818 & 40.315 \\
\hline 总计 & & 35213638 & 1089623 & 100.000 & 100.000 \\
\hline
\end{tabular}


$\mathrm{mV}$

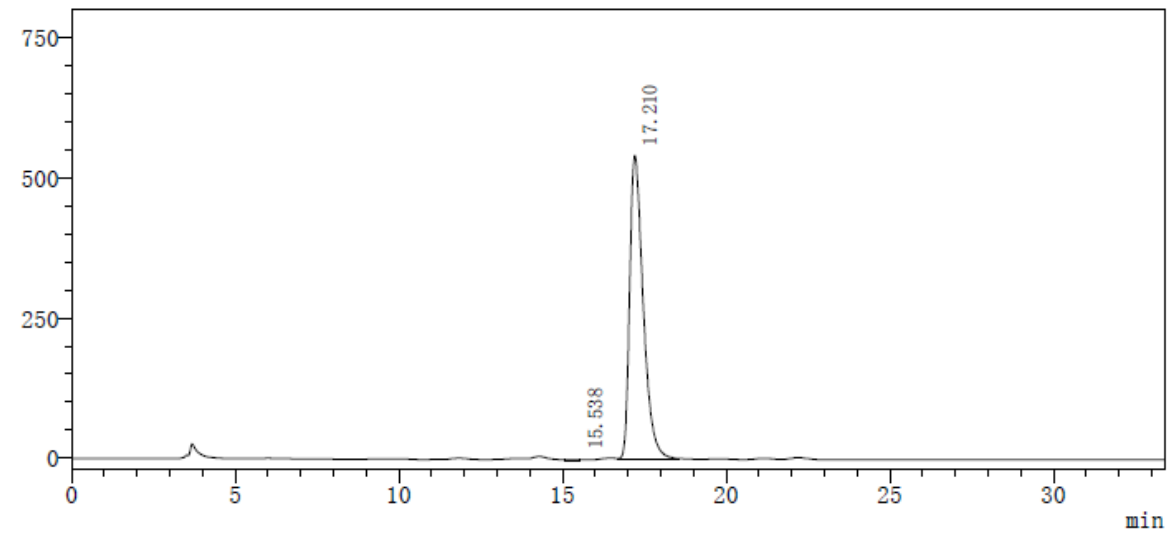

检测器A $220 \mathrm{~nm}$
\begin{tabular}{|c|c|c|c|c|c|}
\hline Peak\# & Time & Area & Height & Height $\%$ & Area\% \\
\hline 1 & 15.538 & 26593 & 1050 & 0.193 & 0.169 \\
\hline 2 & 17.210 & 15672437 & 542384 & 99.807 & 99.831 \\
\hline 总计 & & 15699030 & 543434 & 100.000 & 100.000 \\
\hline
\end{tabular}<smiles>CC(=O)C(C)(N)C(C)/C=C/c1ccccc1F</smiles>

$(2 R, 3 R)-3 \mathbf{d a}$

\section{(2R,3R,E)-methyl 2-amino-5-(2-fluorophenyl)-2,3-dimethylpent-4-enoate}

Prepared according to General Procedure A using Pd-6 and $(R, R p)$-L2-Cu, Purification by flash chromatography $(\mathrm{PE} / \mathrm{EA}=5: 1$ to $1: 1)$ afforded the product as a pale yellow oil, $39.1 \mathrm{mg}, 78 \%$ yield, $12: 1 \mathrm{dr}, 97 \%$ ee, $\mathrm{R}_{\mathrm{f}}=0.35$ (PE/EA, 1:1).

$[\alpha]^{25} \mathrm{D}=38.9\left(\mathrm{c} 0.5, \mathrm{CHCl}_{3}\right)$

${ }^{1} \mathbf{H}$ NMR (400 MHz, CDCl $) \delta 7.41(\mathrm{td}, J=7.7,1.5 \mathrm{~Hz}, 1 \mathrm{H}), 7.18(\mathrm{dd}, J=15.0,5.3 \mathrm{~Hz}, 1 \mathrm{H}), 7.07(\mathrm{t}$, $J=7.2 \mathrm{~Hz}, 1 \mathrm{H}), 7.01(\mathrm{dd}, J=10.1,8.9 \mathrm{~Hz}, 1 \mathrm{H}), 6.57(\mathrm{~d}, J=16.0 \mathrm{~Hz}, 1 \mathrm{H}), 6.19(\mathrm{dd}, J=16.0,8.6 \mathrm{~Hz}$, $1 \mathrm{H}), 3.72(\mathrm{~s}, 3 \mathrm{H}), 2.77-2.62(\mathrm{~m}, 1 \mathrm{H}), 1.68(\mathrm{~s}, 2 \mathrm{H}), 1.33(\mathrm{~s}, 3 \mathrm{H}), 1.13(\mathrm{~d}, J=6.9 \mathrm{~Hz}, 3 \mathrm{H})$.

${ }^{13}$ C NMR (101 MHz, CDCl $) \delta$ 177.41, $160.04(\mathrm{~d}, J=248.3 \mathrm{~Hz}), 133.62(\mathrm{~d}, J=4.3 \mathrm{~Hz}), 128.54(\mathrm{~d}, J$ $=8.2 \mathrm{~Hz}), 127.23(\mathrm{~d}, J=3.6 \mathrm{~Hz}), 125.04(\mathrm{~d}, J=12.4 \mathrm{~Hz}), 124.03(\mathrm{~d}, J=3.4 \mathrm{~Hz}), 123.78(\mathrm{~d}, J=3.7$ $\mathrm{Hz}), 115.62(\mathrm{~d}, J=22.3 \mathrm{~Hz}), 61.02,52.13,46.00,23.53,14.25$.

${ }^{19} \mathrm{~F}$ NMR $\left(376 \mathrm{MHz}, \mathrm{CDCl}_{3}\right) \delta-123.63$

HRMS (ESI) calcd. for $\mathrm{C}_{14} \mathrm{H}_{19} \mathrm{FNO}_{2}{ }^{+}(\mathrm{M}+\mathrm{H})^{+}: 252.1400$, Found: 252.1393

the ee value $\mathrm{was} 97 \%, \mathrm{t}_{\mathrm{r}}($ major $)=20.929 \mathrm{~min}, \mathrm{t}_{\mathrm{r}}($ minor $)=22.571 \mathrm{~min}($ Chiralcel $\mathrm{IC}, \lambda=220 \mathrm{~nm}$, hexanes $:{ }^{i} \mathrm{PrOH}=98: 2$, flow rate $\left.=1.0 \mathrm{~mL} / \mathrm{min}\right)$. 
$\mathrm{mV}$

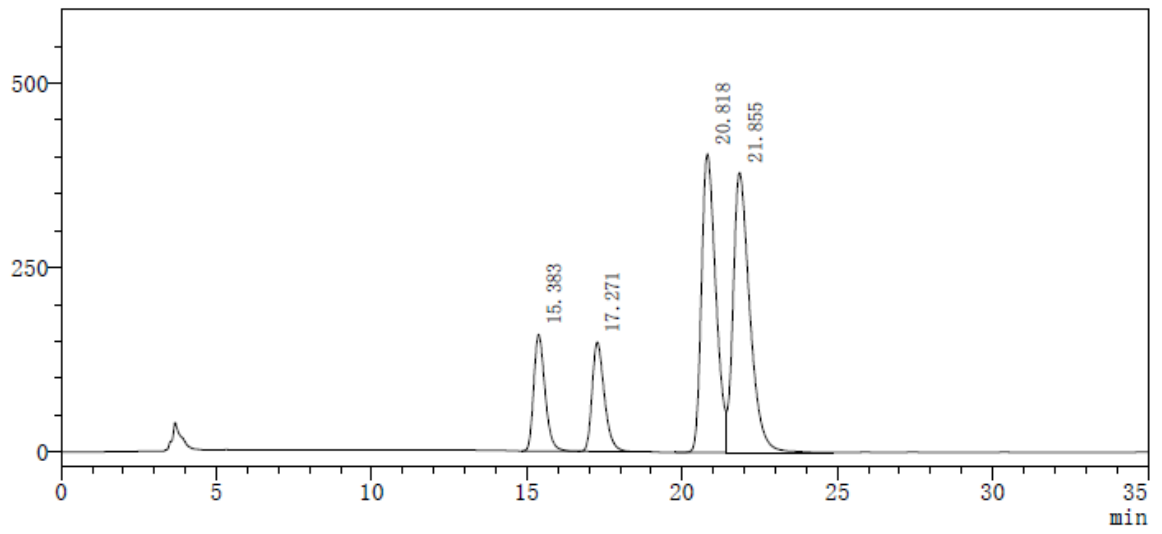

检测器A 220nm
\begin{tabular}{|c|c|c|c|c|c|}
\hline Peak\# & Time & Area & Height & Height\% & Area\% \\
\hline 1 & 15.383 & 4007779 & 157761 & 14.479 & 11.381 \\
\hline 2 & 17.271 & 4075638 & 148191 & 13.600 & 11.574 \\
\hline 3 & 20.818 & 12933755 & 404280 & 37.103 & 36.729 \\
\hline 4 & 21.855 & 14196467 & 379390 & 34.818 & 40.315 \\
\hline 总计 & 35213638 & 1089623 & 100.000 & 100.000 \\
\hline
\end{tabular}

$\mathrm{mV}$

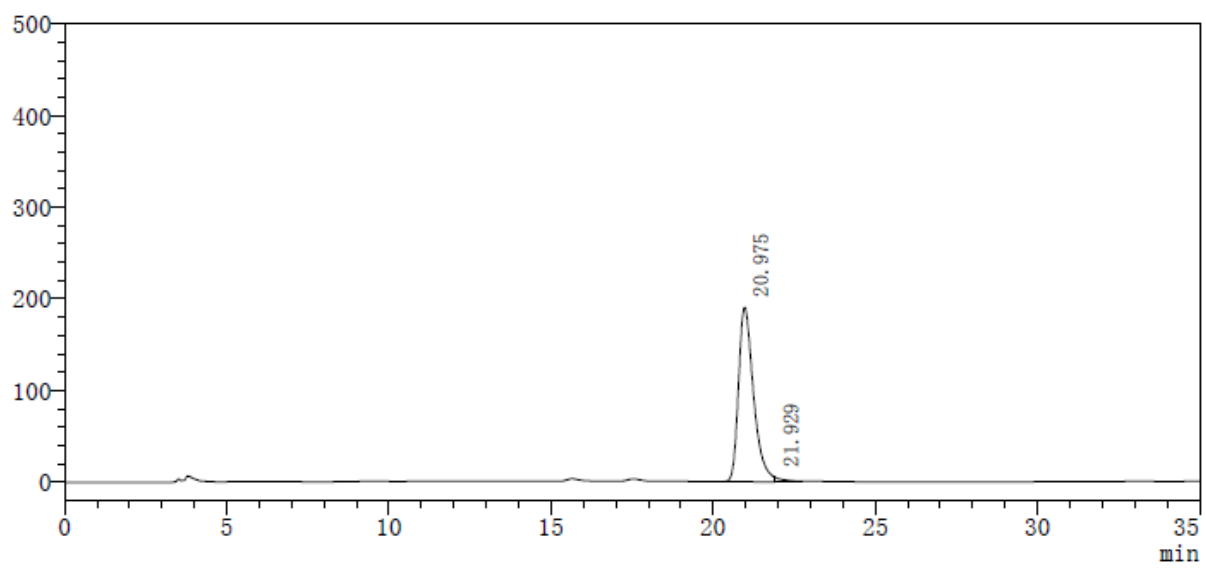

检测器A $220 \mathrm{~nm}$
\begin{tabular}{|c|c|c|c|c|c|}
\hline Peak\# & Time & Area & Height & Height\% & Area\% \\
\hline 1 & 20.975 & 6093964 & 190122 & 97.593 & 98.512 \\
\hline 2 & 21.929 & 92054 & 4690 & 2.407 & 1.488 \\
\hline 总计 & & 6186018 & 194812 & 100.000 & 100.000 \\
\hline
\end{tabular}<smiles>[M]C(C=Cc1cccc(F)c1)C(C)(N)C(C)=O</smiles>

(2S,3R,E)-methyl 2-amino-5-(3-fluorophenyl)-2,3-dimethylpent-4-enoate

Prepared according to General Procedure A using Pd-6 and $(S, S p)$-L2-Cu, Purification by flash chromatography $(\mathrm{PE} / \mathrm{EA}=5: 1$ to $1: 1)$ afforded the product as a pale yellow oil, $41.2 \mathrm{mg}, 82 \%$ yield, $>20: 1 \mathrm{dr},>99 \%$ ee, $\mathrm{R}_{\mathrm{f}}=0.4(\mathrm{PE} / \mathrm{EA}, 1: 1)$.

$[\alpha]^{25} \mathrm{D}=50.3\left(\mathrm{c} 0.5, \mathrm{CHCl}_{3}\right)$ 
${ }^{1}$ H NMR (400 MHz, CDCl $) \delta 7.32-7.23(\mathrm{~m}, 1 \mathrm{H}), 7.10(\mathrm{dd}, J=19.2,8.9 \mathrm{~Hz}, 2 \mathrm{H}), 6.90(\mathrm{t}, J=8.3$ $\mathrm{Hz}, 1 \mathrm{H}), 6.41(\mathrm{~d}, J=15.7 \mathrm{~Hz}, 1 \mathrm{H}), 6.19(\mathrm{dd}, J=16.4,8.4 \mathrm{~Hz}, 1 \mathrm{H}), 3.73(\mathrm{~s}, 3 \mathrm{H}), 2.73-2.56(\mathrm{~m}, 1 \mathrm{H})$, $1.65(\mathrm{~s}, 2 \mathrm{H}), 1.31(\mathrm{~s}, 3 \mathrm{H}), 1.05(\mathrm{~d}, J=6.7 \mathrm{~Hz}, 3 \mathrm{H})$.

${ }^{13}$ C NMR (101 MHz, CDCl $) \delta 177.77,163.09(\mathrm{~d}, J=245.1 \mathrm{~Hz}), 139.67(\mathrm{~d}, J=7.7 \mathrm{~Hz}), 132.18$, $130.73(\mathrm{~d}, J=2.5 \mathrm{~Hz}), 129.92(\mathrm{~d}, J=8.4 \mathrm{~Hz}), 122.11(\mathrm{~d}, J=2.7 \mathrm{~Hz}), 114.01(\mathrm{~d}, J=21.4 \mathrm{~Hz}), 112.64$ $(\mathrm{d}, J=21.7 \mathrm{~Hz}), 60.61,52.22,45.79,24.80,15.66$.

${ }^{19}$ F NMR (376 MHz, CDCl 3$) \delta-118.65$

HRMS (ESI) calcd. for $\mathrm{C}_{14} \mathrm{H}_{19} \mathrm{FNO}_{2}{ }^{+}(\mathrm{M}+\mathrm{H})^{+}:$252.1400, Found: 252.1397

the ee value $\mathrm{was}>99 \%, \mathrm{t}_{\mathrm{r}}($ minor $)=15.408 \mathrm{~min}, \mathrm{t}_{\mathrm{r}}$ (major) $=17.413 \mathrm{~min}($ Chiralcel $\mathrm{IC}, \lambda=220 \mathrm{~nm}$, hexanes $:{ }^{i} \mathrm{PrOH}=98: 2$, flow rate $\left.=1.0 \mathrm{~mL} / \mathrm{min}\right)$.

$\mathrm{mV}$

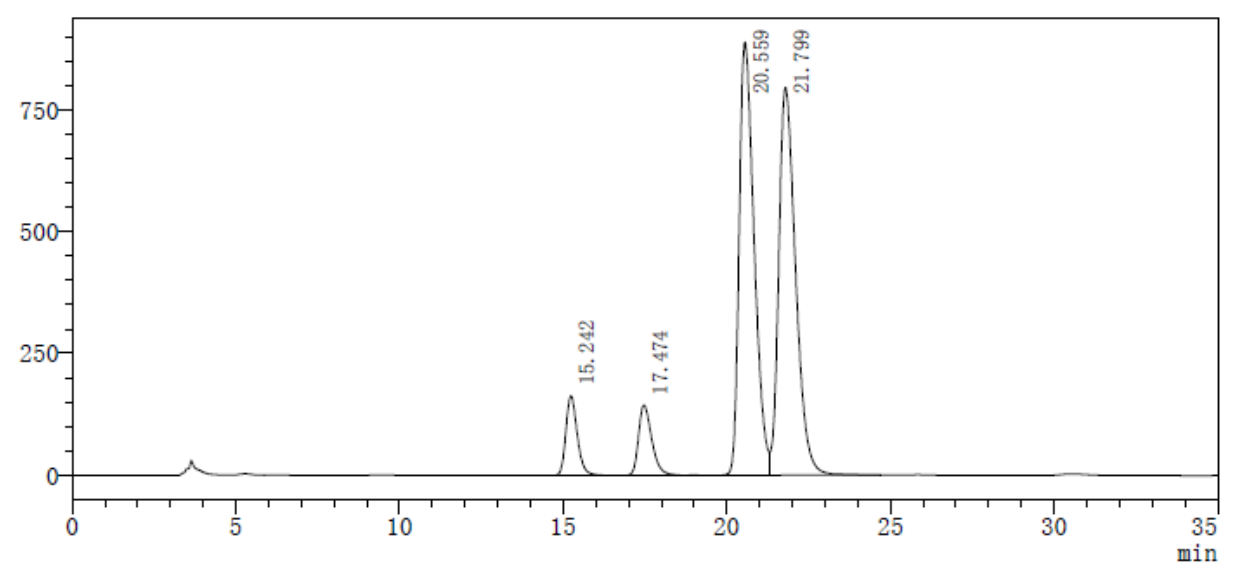

\begin{tabular}{|c|c|c|c|c|c|}
\hline Peak\# & Time & Area & Height & Height\% & Area\% \\
\hline 1 & 15.242 & 3954398 & 163106 & 8.190 & 6.097 \\
\hline 2 & 17. 474 & 4089637 & 144497 & 7.255 & 6.306 \\
\hline 3 & 20.559 & 28289463 & 888387 & 44.606 & 43.617 \\
\hline 4 & 21.799 & 28524711 & 795639 & 39.949 & 43.980 \\
\hline 总计 & & 64858209 & 1991630 & 100.000 & 100.000 \\
\hline
\end{tabular}


$\mathrm{mV}$

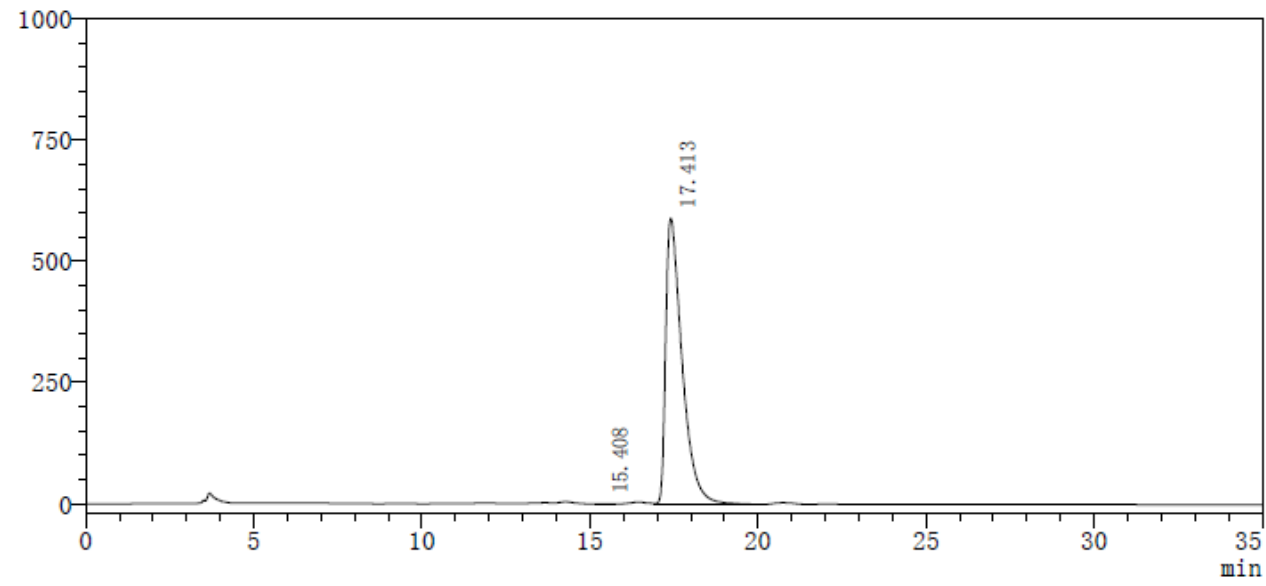

检测器A $220 \mathrm{~nm}$
\begin{tabular}{|c|c|c|c|c|c|}
\hline Peak\# & Time & Area & Height & Height\% & Area\% \\
\hline 1 & 15.408 & 17944 & 542 & 0.092 & 0.090 \\
\hline 2 & 17.413 & 19987697 & 589271 & 99.908 & 99.910 \\
\hline 总计 & & 20005641 & 589813 & 100.000 & 100.000 \\
\hline
\end{tabular}<smiles>COC(=O)[C@](C)(N)/C=C/c1ccc(F)cc1</smiles>

$(2 S, 3 R)-3 f a$

\section{(2S,3R,E)-methyl 2-amino-5-(4-fluorophenyl)-2,3-dimethylpent-4-enoate}

Prepared according to General Procedure A using Pd-6 and $(S, S p)$-L2-Cu, Purification by flash chromatography (PE/EA $=5: 1$ to 1:1) afforded the product as a pale yellow solid using Pd-6 and $(S, S p)$-L2-Cu, m.p. $97-99{ }^{\circ} \mathrm{C}, 35 \mathrm{mg}, 70 \%$ yield, $>20: 1 \mathrm{dr},>99 \%$ ee, $\mathrm{R}_{\mathrm{f}}=0.4$ (PE/EA, 1:1). $[\alpha]^{25} \mathrm{D}=69.7\left(\mathrm{c} 1.0, \mathrm{CHCl}_{3}\right)$

${ }^{1}$ H NMR (400 MHz, CDCl 3 ) $\delta 7.33(\mathrm{dd}, J=8.5,5.5 \mathrm{~Hz}, 2 \mathrm{H}), 6.99(\mathrm{t}, J=8.7 \mathrm{~Hz}, 2 \mathrm{H}), 6.41(\mathrm{~d}, J=$ $15.8 \mathrm{~Hz}, 1 \mathrm{H}), 6.08(\mathrm{dd}, J=15.8,9.1 \mathrm{~Hz}, 1 \mathrm{H}), 3.73(\mathrm{~s}, 3 \mathrm{H}), 2.82-2.41(\mathrm{~m}, 1 \mathrm{H}), 1.66(\mathrm{~s}, 2 \mathrm{H}), 1.31(\mathrm{~s}$, $3 \mathrm{H}), 1.05(\mathrm{~d}, J=6.9 \mathrm{~Hz}, 3 \mathrm{H})$.

${ }^{13}$ C NMR (101 MHz, CDCl $) \delta 177.83,162.11(\mathrm{~d}, J=246.1 \mathrm{~Hz}), 133.46(\mathrm{~d}, J=3.3 \mathrm{~Hz}), 130.60$, $130.38(\mathrm{~d}, J=1.6 \mathrm{~Hz}), 127.67(\mathrm{~d}, J=7.7 \mathrm{~Hz}), 115.36(\mathrm{~d}, J=21.6 \mathrm{~Hz}), 60.65,52.19,45.83,24.81$, 15.73 .

${ }^{19}$ F NMR (376 MHz, CDCl 3 ) $\delta-120.03$

HRMS (ESI) calcd. for $\mathrm{C}_{14} \mathrm{H}_{19} \mathrm{FNO}_{2}{ }^{+}(\mathrm{M}+\mathrm{H})^{+}: 252.1400$, Found: 252.1398

the ee value was $>99 \%, \mathrm{t}_{\mathrm{r}}($ minor $)=15.154 \mathrm{~min}, \mathrm{t}_{\mathrm{r}}($ major $)=17.498 \mathrm{~min}($ Chiralcel $\mathrm{IC}, \lambda=220 \mathrm{~nm}$, hexanes $:{ }^{i} \mathrm{PrOH}=98: 2$, flow rate $=1.0 \mathrm{~mL} / \mathrm{min}$ ). 
$\mathrm{mV}$

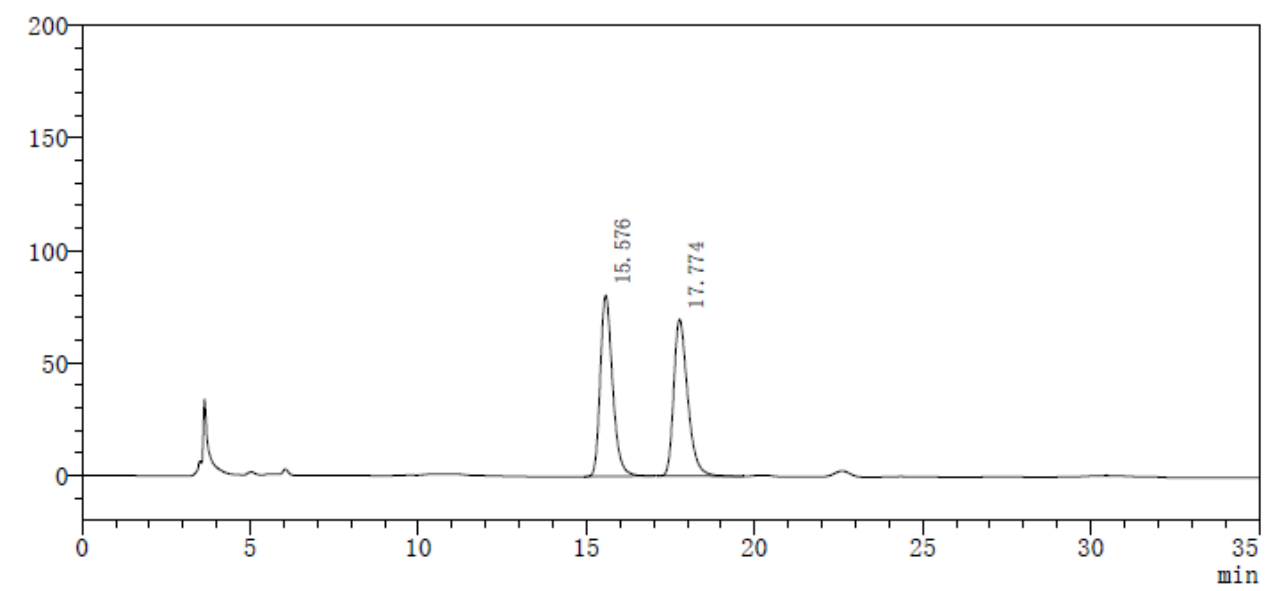

检测器A $220 \mathrm{~nm}$
\begin{tabular}{|c|c|c|c|c|c|}
\hline Peak\# & Time & Area & Height & Height\% & Area\% \\
\hline 1 & 15.576 & 2052838 & 80430 & 53.574 & 50.533 \\
\hline 2 & 17.774 & 2009532 & 69700 & 46.426 & 49.467 \\
\hline 总计 & & 4062371 & 150129 & 100.000 & 100.000 \\
\hline
\end{tabular}

$\mathrm{mV}$

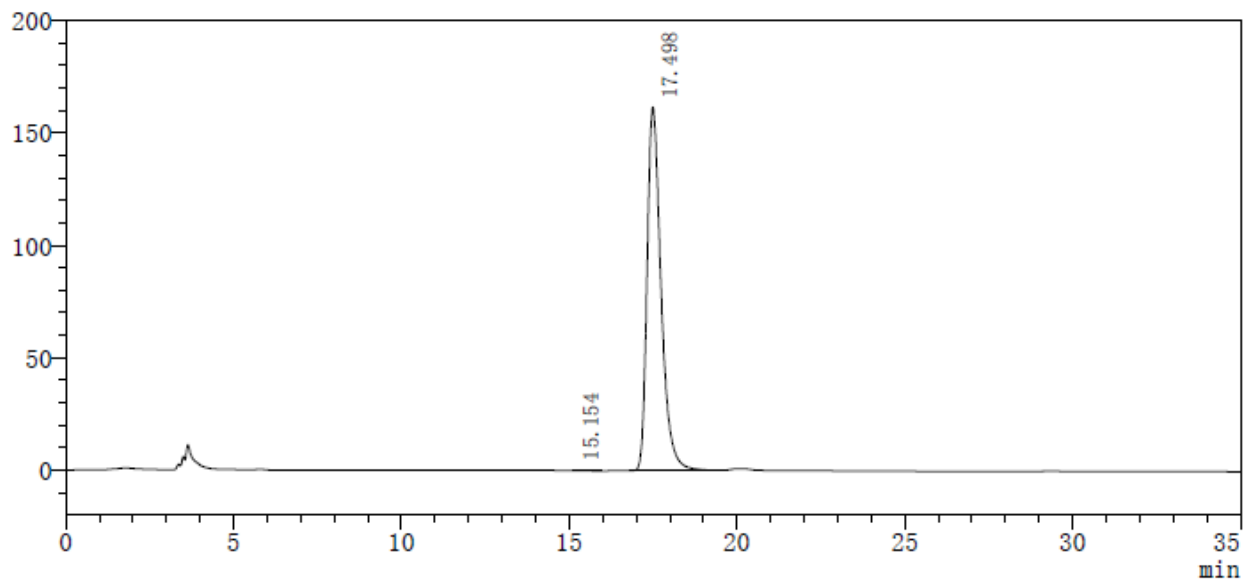

检测器A $220 \mathrm{~nm}$

\begin{tabular}{|c|c|c|c|c|c|}
\hline Peak\# & Time & Area & Height & Height\% & Area\% \\
\hline 1 & 15.154 & 1721 & 73 & 0.045 & 0.037 \\
\hline 2 & 17.498 & 4686545 & 161635 & 99.955 & 99.963 \\
\hline 总计 & & 4688265 & 161709 & 100.000 & 100.000 \\
\hline
\end{tabular}<smiles>CC(=O)C(C)(N)[C@@H](C)/C=C/c1ccc(F)cc1</smiles>

$(2 R, 3 R)-3 f a$

(2R,3R,E)-methyl 2-amino-5-(4-fluorophenyl)-2,3-dimethylpent-4-enoate

Prepared according to General Procedure A using Pd-6 and $(R, R p)$-L2-Cu, Purification by flash chromatography (PE/EA $=5: 1$ to 1:1) afforded the product as a pale yellow solid using Pd-6 and $(R, R p)$-L2-Cu, m.p. $64-65^{\circ} \mathrm{C}, 33 \mathrm{mg}, 66 \%$ yield, $14: 1 \mathrm{dr},>99 \%$ ee, $\mathrm{R}_{\mathrm{f}}=0.35$ (PE/EA, 1:1). $[\alpha]^{25} \mathrm{D}=44.9\left(\mathrm{c} 0.4, \mathrm{CHCl}_{3}\right)$ 
${ }^{1}$ H NMR (400 MHz, CDCl 3$) \delta 7.30(\mathrm{dd}, J=8.4,5.6 \mathrm{~Hz}, 2 \mathrm{H}), 6.98(\mathrm{t}, J=8.6 \mathrm{~Hz}, 2 \mathrm{H}), 6.37(\mathrm{~d}, J=$ $15.8 \mathrm{~Hz}, 1 \mathrm{H}), 6.03(\mathrm{dd}, J=15.8,8.5 \mathrm{~Hz}, 1 \mathrm{H}), 3.72(\mathrm{~s}, 3 \mathrm{H}), 2.85-2.54(\mathrm{~m}, 1 \mathrm{H}), 1.74(\mathrm{~s}, 2 \mathrm{H}), 1.33$ (s, $3 \mathrm{H}), 1.11(\mathrm{~d}, J=6.8 \mathrm{~Hz}, 3 \mathrm{H})$.

${ }^{13}$ C NMR (101 MHz, CDCl $) \delta$ 177.32, $162.15(\mathrm{~d}, J=246.3 \mathrm{~Hz}), 133.41(\mathrm{~d}, J=3.1 \mathrm{~Hz}), 130.57(\mathrm{~d}, J$ $=1.2 \mathrm{~Hz}), 130.28,127.69(\mathrm{~d}, J=8.1 \mathrm{~Hz}), 115.37(\mathrm{~d}, J=21.6 \mathrm{~Hz}), 61.03,52.15,45.53,23.46,14.35$.

${ }^{19}$ F NMR (376 MHz, $\left.\mathbf{C D C l}_{3}\right) \delta-119.93$

HRMS (ESI) calcd. for $\mathrm{C}_{14} \mathrm{H}_{19} \mathrm{FNO}_{2}{ }^{+}(\mathrm{M}+\mathrm{H})^{+}: 252.1400$, Found: 252.1395

the ee value $\mathrm{was}>99 \%, \mathrm{t}_{\mathrm{r}}($ minor $)=20.138 \mathrm{~min}, \mathrm{t}_{\mathrm{r}}($ major $)=21.834 \mathrm{~min}($ Chiralcel $\mathrm{IC}, \lambda=220 \mathrm{~nm}$, hexanes : ${ }^{i} \mathrm{PrOH}=98: 2$, flow rate $=1.0 \mathrm{~mL} / \mathrm{min}$ ).

$\mathrm{mV}$

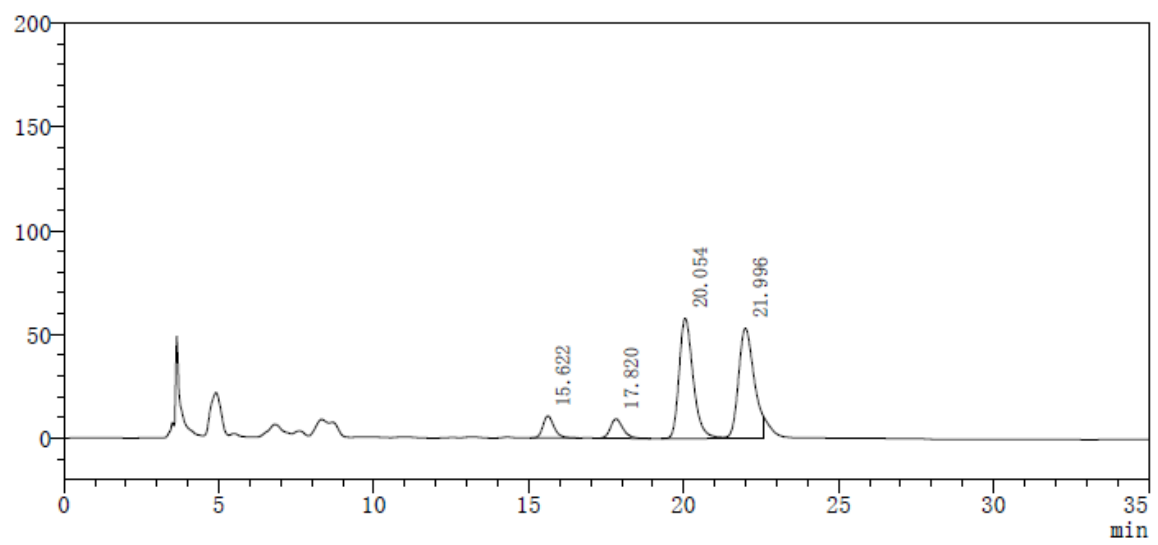

检测器A $220 \mathrm{~nm}$
\begin{tabular}{|c|c|c|c|c|c|}
\hline Peak\# & Time & Area & Height & Height\% & Area\% \\
\hline 1 & 15.622 & 258959 & 10547 & 8.065 & 6.168 \\
\hline 2 & 17.820 & 260794 & 9276 & 7.093 & 6.212 \\
\hline 3 & 20.054 & 1818891 & 57988 & 44.339 & 43.325 \\
\hline 4 & 21.996 & 1859601 & 52971 & 40.503 & 44.295 \\
\hline 总计 & & 4198245 & 130782 & 100.000 & 100.000 \\
\hline
\end{tabular}

$\mathrm{mV}$

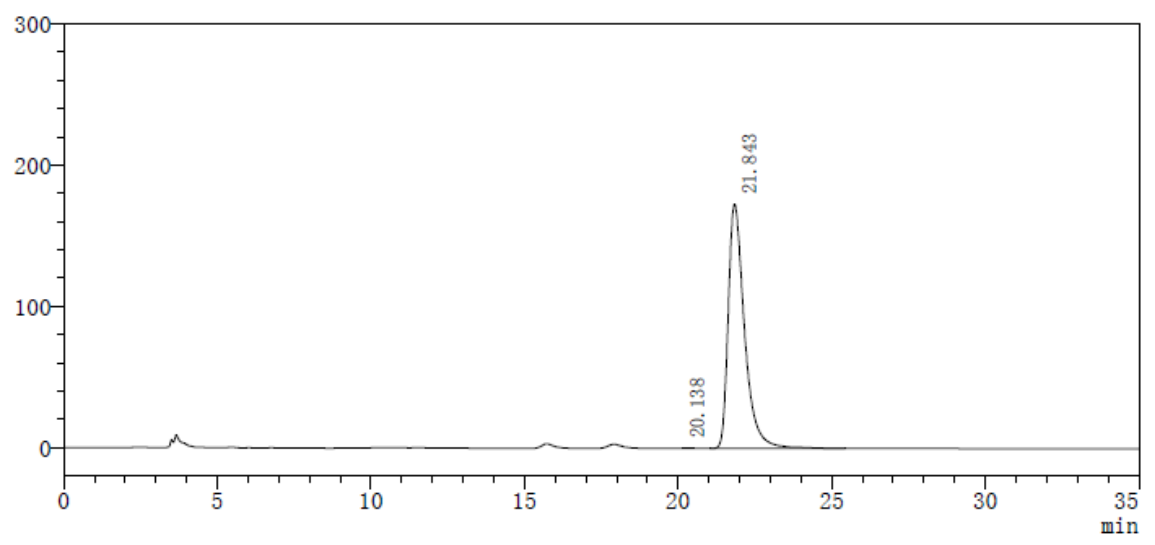

检测器A $220 \mathrm{~nm}$
\begin{tabular}{|c|c|c|c|c|c|}
\hline Peak\# & Time & Area & Height & Height $\%$ & Area $\%$ \\
\hline 1 & 20.138 & 1204 & 78 & 0.045 & 0.019 \\
\hline 2 & 21.843 & 6376001 & 172965 & 99.955 & 99.981 \\
\hline 总计 & & 6377204 & 173043 & 100.000 & 100.000 \\
\hline
\end{tabular}


<smiles>COC(=O)C(C)(N)C(C)/C=C/c1ccc(Cl)cc1</smiles>

\section{(2S,3R,E)-methyl 2-amino-5-(4-chlorophenyl)-2,3-dimethylpent-4-enoate}

Prepared according to General Procedure A using Pd-6 and $(S, S p)$-L2-Cu, Purification by flash chromatography ( $\mathrm{PE} / \mathrm{EA}=5: 1$ to 1:1) afforded the product as a pale yellow solid, $38 \mathrm{mg}, 72 \%$ yield, m.p. $92-93{ }^{\circ} \mathrm{C},>20: 1 \mathrm{dr},>99 \%$ ee, $\mathrm{R}_{\mathrm{f}}=0.4(\mathrm{PE} / \mathrm{EA}, 1: 1)$.

$[\alpha]^{25} \mathrm{D}=63.3\left(\mathrm{c} \quad 0.5, \mathrm{CHCl}_{3}\right)$

${ }^{1}$ H NMR (400 MHz, CDCl 3 ) $\delta 7.37-7.17(\mathrm{~m}, 4 \mathrm{H}), 6.40(\mathrm{~d}, J=15.8 \mathrm{~Hz}, 1 \mathrm{H}), 6.15(\mathrm{dd}, J=15.8,9.1$ $\mathrm{Hz}, 1 \mathrm{H}), 3.73(\mathrm{~s}, 3 \mathrm{H}), 2.71-2.58(\mathrm{~m}, 1 \mathrm{H}), 1.63(\mathrm{~s}, 2 \mathrm{H}), 1.30(\mathrm{~s}, 3 \mathrm{H}), 1.04(\mathrm{~d}, J=6.8 \mathrm{~Hz}, 3 \mathrm{H})$.

${ }^{13}$ C NMR (101 MHz, CDCl $) \delta$ 177.81, 135.78, 132.80, 131.40, 130.59, 128.64, 127.44, 60.65, 52.24, $45.84,24.82,15.71$.

HRMS (ESI) calcd. for $\mathrm{C}_{14} \mathrm{H}_{19} \mathrm{ClNO}_{2}{ }^{+}(\mathrm{M}+\mathrm{H})^{+}:$: 268.1104, Found: 268.1099

the ee value $\mathrm{was}>99 \%, \mathrm{t}_{\mathrm{r}}($ minor $)=16.574 \mathrm{~min}, \mathrm{t}_{\mathrm{r}}$ (major) $=17.649 \min ($ Chiralcel $\mathrm{IC}, \lambda=220 \mathrm{~nm}$, hexanes $:{ }^{i} \mathrm{PrOH}=98: 2$, flow rate $\left.=1.0 \mathrm{~mL} / \mathrm{min}\right)$.

$\mathrm{mV}$

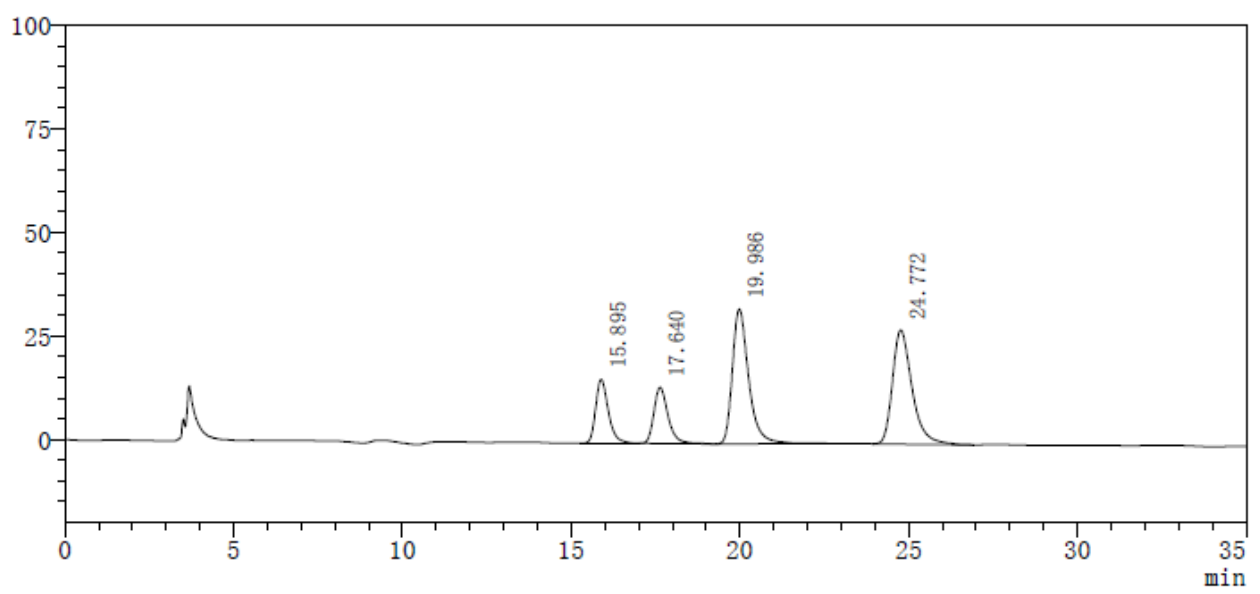

\begin{tabular}{|c|c|c|c|c|c|}
\hline Peak\# & Time & Area & Height & Height\% & Area\% \\
\hline 1 & 15.895 & 405566 & 15449 & 17.345 & 13.481 \\
\hline 2 & 17.640 & 397063 & 13581 & 15.247 & 13.199 \\
\hline 3 & 19.986 & 1094142 & 32520 & 36.510 & 36.370 \\
\hline 4 & 24.772 & 1111595 & 27522 & 30.899 & 36.950 \\
\hline 总计 & & 3008366 & 89073 & 100.000 & 100.000 \\
\hline
\end{tabular}


$\mathrm{mV}$

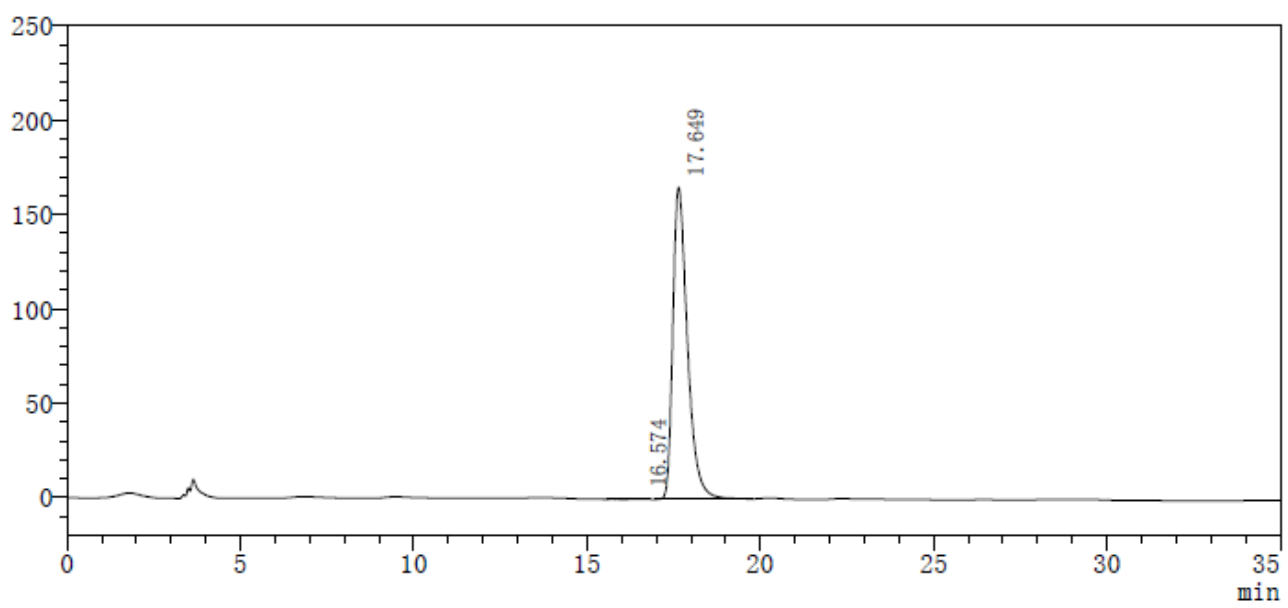

检测器A 220 nm
\begin{tabular}{|c|c|c|c|c|c|}
\hline Peak\# & Time & Area & Height & Height $\%$ & Area $\%$ \\
\hline 1 & 16.574 & 3092 & 47 & 0.028 & 0.064 \\
\hline 2 & 17.649 & 4841042 & 165084 & 99.972 & 99.936 \\
\hline 总计 & & 4844134 & 165131 & 100.000 & 100.000 \\
\hline
\end{tabular}<smiles>COC(=O)[C@](C)(N)[C@@H](C)/C=C/c1ccc(C(F)(F)F)cc1</smiles>

(2S, 3R)-3ha

(2S,3R,E)-methyl 2-amino-2,3-dimethyl-5-(4-(trifluoromethyl)phenyl)pent-4-enoate

Prepared according to General Procedure A using Pd-6 and $(S, S p)-\mathbf{L 2}-\mathbf{C u}$, Purification by flash chromatography (PE/EA $=5: 1$ to $1: 1)$ afforded the product as a pale yellow solid, $42 \mathrm{mg}, 70 \%$ yield, m.p. $87-88^{\circ} \mathrm{C},>20: 1 \mathrm{dr},>99 \%$ ee, $\mathrm{R}_{\mathrm{f}}=0.3(\mathrm{PE} / \mathrm{EA}, 1: 1)$.

$[\alpha]^{25}=49.6\left(\mathrm{c} 1.0, \mathrm{CHCl}_{3}\right)$

${ }^{1} \mathbf{H}$ NMR $\left(400 \mathrm{MHz}, \mathbf{C D C l}_{3}\right) \delta 7.55(\mathrm{~d}, J=8.0 \mathrm{~Hz}, 2 \mathrm{H}), 7.46(\mathrm{~d}, J=8.0 \mathrm{~Hz}, 2 \mathrm{H}), 6.48(\mathrm{~d}, J=15.9 \mathrm{~Hz}$, $1 \mathrm{H}), 6.30(\mathrm{dd}, J=15.9,9.1 \mathrm{~Hz}, 1 \mathrm{H}), 3.74(\mathrm{~s}, 3 \mathrm{H}), 2.93-2.47(\mathrm{~m}, 1 \mathrm{H}), 1.69$ (s, 2H), $1.31(\mathrm{~s}, 3 \mathrm{H}), 1.06$ $(\mathrm{d}, J=6.8 \mathrm{~Hz}, 3 \mathrm{H})$.

${ }^{13}$ C NMR (101 MHz, CDCl $) \delta 177.88,140.74(\mathrm{~d}, J=1.2 \mathrm{~Hz}), 133.58,130.55,129.04(\mathrm{dd}, J=64.8$, $32.4 \mathrm{~Hz}$ ), 126.37, 125.47 (q, $J=3.8 \mathrm{~Hz}), 60.81,52.29,45.86,29.72,24.78,15.65$.

${ }^{19}$ F NMR (376 MHz, $\left.\mathbf{C D C l}_{3}\right) \delta-67.09$.

HRMS (ESI) calcd. for $\mathrm{C}_{15} \mathrm{H}_{19} \mathrm{~F}_{3} \mathrm{NO}_{2}{ }^{+}(\mathrm{M}+\mathrm{H})^{+}: 302.1368$, Found: 302.1304

the ee value was $>99 \%, \mathrm{t}_{\mathrm{r}}($ minor $)=10.858 \mathrm{~min}, \mathrm{t}_{\mathrm{r}}$ (major) $=11.446 \mathrm{~min}$ (Chiralcel $\mathrm{IC}, \lambda=220 \mathrm{~nm}$, hexanes $:{ }^{i} \mathrm{PrOH}=98: 2$, flow rate $\left.=1.0 \mathrm{~mL} / \mathrm{min}\right)$. 
$\mathrm{mV}$

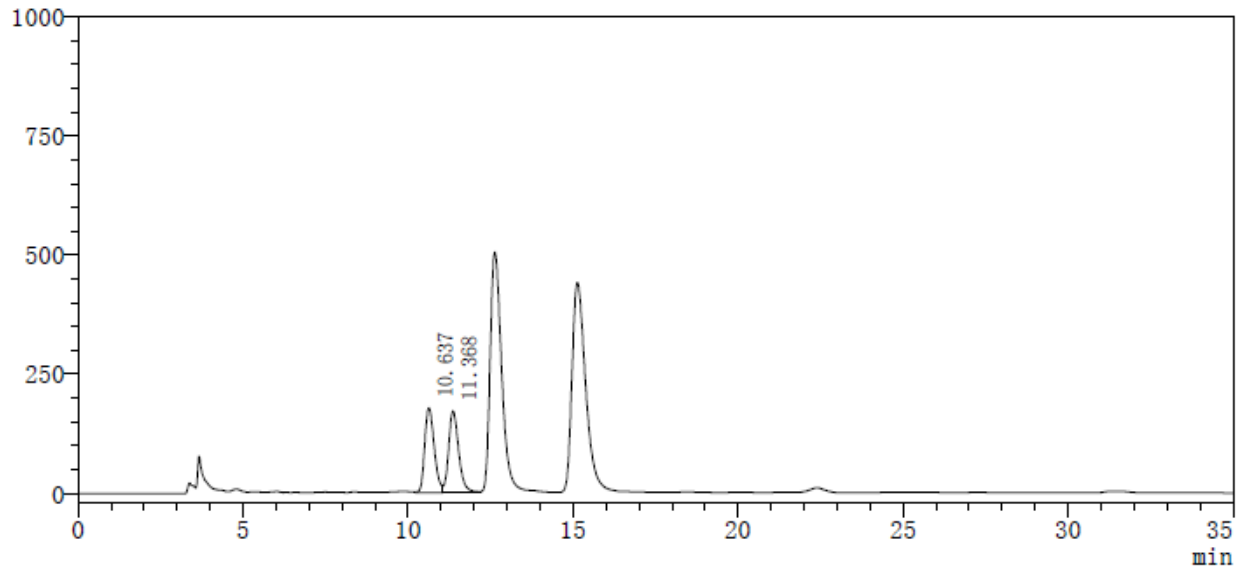

检测器A $220 \mathrm{~nm}$
\begin{tabular}{|c|c|c|c|c|c|}
\hline Peak\# & Time & Area & Height & Height\% & Area\% \\
\hline 1 & 10.637 & 3595147 & 178286 & 51.056 & 49.661 \\
\hline 2 & 11.368 & 3644261 & 170914 & 48.944 & 50.339 \\
\hline 总计 & & 7239408 & 349200 & 100.000 & 100.000 \\
\hline
\end{tabular}

$\mathrm{mV}$

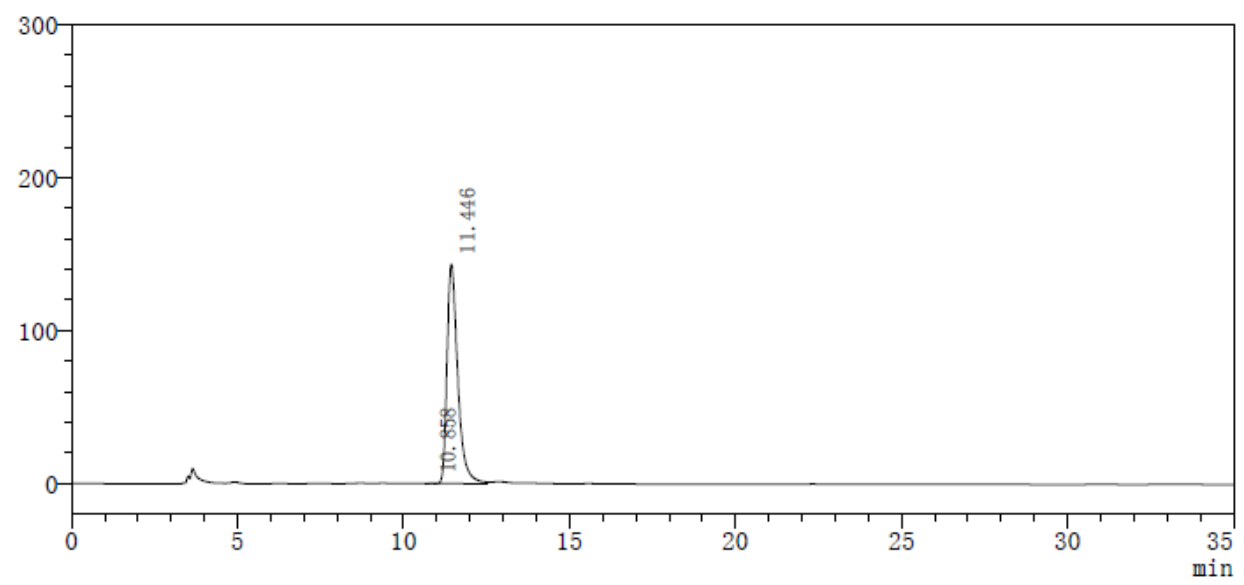

\begin{tabular}{|c|c|c|c|c|c|}
\hline Peak\# & Time & Area & H & Hejght\% & Area\% \\
\hline 1 & 10.858 & 418 & 32 & 0.022 & 0.013 \\
\hline 2 & 11.446 & 3239094 & 143488 & 99.978 & 99.987 \\
\hline 总计 & & 3239512 & 143520 & 100.000 & 100.000 \\
\hline
\end{tabular}<smiles>COC(=O)[C@](C)(N)[C@@H](C)/C=C/c1ccc(OC)cc1</smiles>

$(2 S, 3 R)-3 i a$

\section{(2S,3R,E)-methyl 2-amino-5-(4-methoxyphenyl)-2,3-dimethylpent-4-enoate}

Prepared according to General Procedure A using Pd-6 and $(S, S p)$-L2-Cu, Purification by flash chromatography (PE/EA $=5: 1$ to 1:2) afforded the product as a pale yellow solid, $41 \mathrm{mg}, 78 \%$ yield, m.p. $74-76{ }^{\circ} \mathrm{C},>20: 1 \mathrm{dr},>99 \%$ ee, $\mathrm{R}_{\mathrm{f}}=0.2(\mathrm{PE} / \mathrm{EA}, 1: 1)$. 
$[\alpha]^{25} \mathrm{D}=74.7\left(\mathrm{c} 0.5, \mathrm{CHCl}_{3}\right)$

${ }^{1}$ H NMR (400 MHz, CDCl $) \delta 7.30(\mathrm{~d}, J=8.6 \mathrm{~Hz}, 2 \mathrm{H}), 6.84(\mathrm{~d}, J=8.6 \mathrm{~Hz}, 2 \mathrm{H}), 6.39(\mathrm{~d}, J=15.8 \mathrm{~Hz}$, $1 \mathrm{H}), 6.01(\mathrm{dd}, J=15.8,9.1 \mathrm{~Hz}, 1 \mathrm{H}), 3.80(\mathrm{~s}, 3 \mathrm{H}), 3.72(\mathrm{~s}, 3 \mathrm{H}), 2.81-2.46(\mathrm{~m}, 1 \mathrm{H}), 1.78(\mathrm{~s}, 2 \mathrm{H}), 1.31$ $(\mathrm{s}, 3 \mathrm{H}), 1.04(\mathrm{~d}, J=6.9 \mathrm{~Hz}, 3 \mathrm{H})$.

${ }^{13}$ C NMR (101 MHz, CDCl $) \delta 177.87,158.97,131.27,130.11,128.23,127.36,113.92,60.75,55.30$, 52.16, 45.91, 24.79, 15.79.

HRMS (ESI) calcd. for $\mathrm{C}_{15} \mathrm{H}_{22} \mathrm{NO}_{3}{ }^{+}(\mathrm{M}+\mathrm{H})^{+}:$264.1600, Found: 264.1594

the ee value $\mathrm{was}>99 \%, \mathrm{t}_{\mathrm{r}}($ minor $)=36,446 \mathrm{~min}, \mathrm{t}_{\mathrm{r}}$ (major) $=45.266 \mathrm{~min}$ (Chiralcel $\mathrm{IC}, \lambda=220 \mathrm{~nm}$, hexanes $:{ }^{i} \mathrm{PrOH}=98: 2$, flow rate $\left.=1.0 \mathrm{~mL} / \mathrm{min}\right)$.

$\mathrm{mV}$

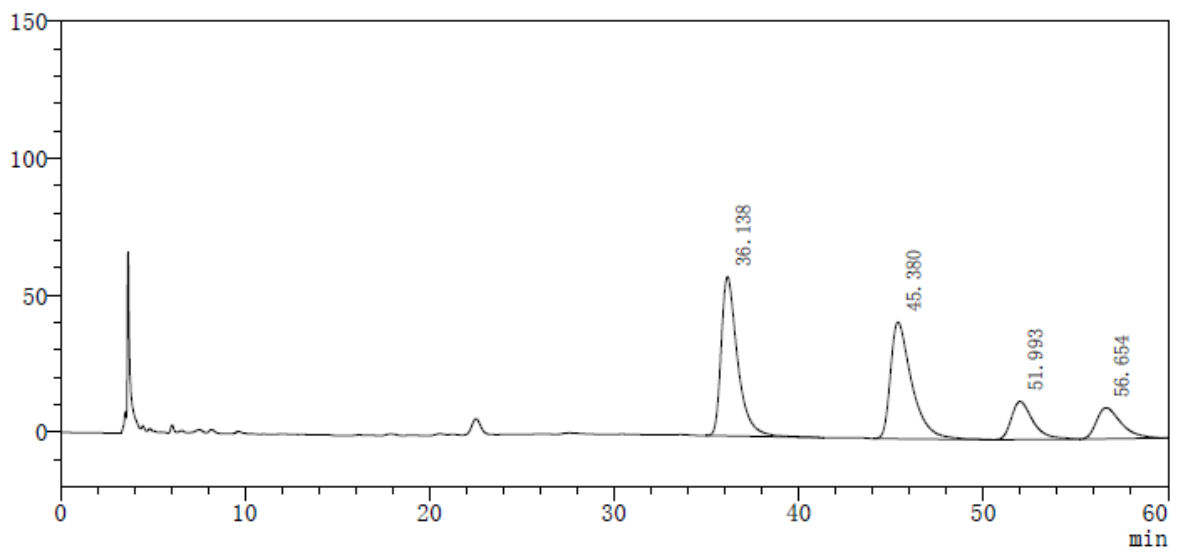

检测器A 220nm
\begin{tabular}{|c|c|c|c|c|c|}
\hline Peak\# & Time & Area & Height & Height\% & Area\% \\
\hline 1 & 36.138 & 3580564 & 58057 & 46.156 & 39.003 \\
\hline 2 & 45.380 & 3411096 & 42563 & 33.838 & 37.157 \\
\hline 3 & 51.993 & 1129619 & 13861 & 11.020 & 12.305 \\
\hline 4 & 56.654 & 1059023 & 11304 & 8.987 & 11.536 \\
\hline 总计 & & 9180302 & 125785 & 100.000 & 100.000 \\
\hline
\end{tabular}

$\mathrm{mV}$

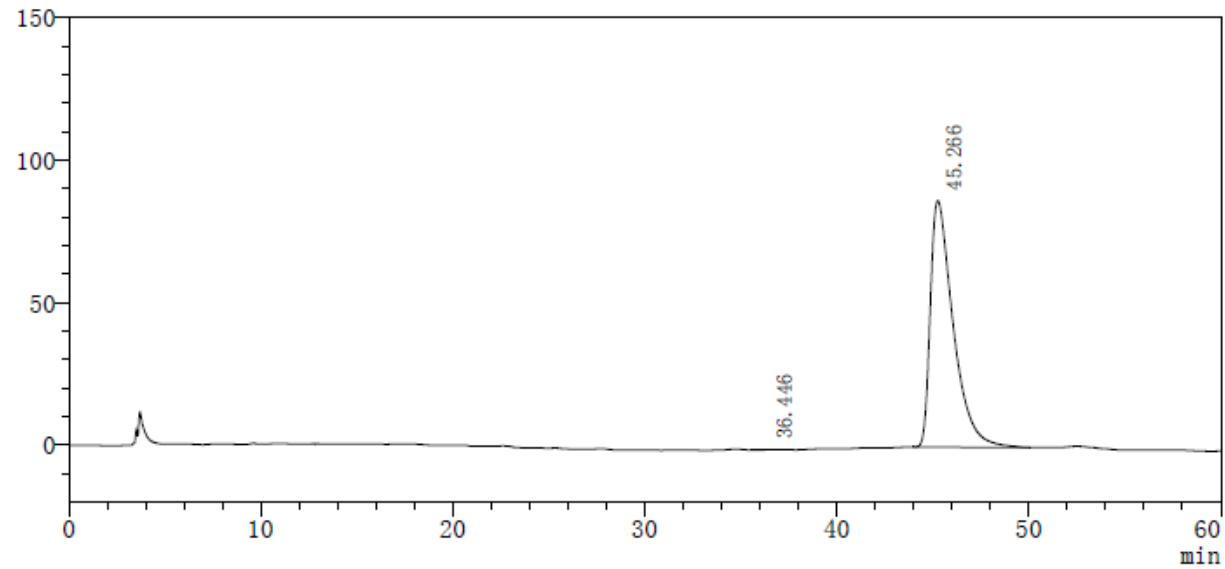

检测器A $220 \mathrm{~nm}$

\begin{tabular}{|c|c|c|c|c|c|}
\hline Peak\# & Time & Area & Height & Height\% & Area\% \\
\hline 1 & 36.446 & 6712 & 182 & 0.210 & 0.094 \\
\hline 2 & 45.266 & 7160179 & 86422 & 99.790 & 99.906 \\
\hline 总计 & & 7166891 & 86603 & 100.000 & 100.000 \\
\hline
\end{tabular}


<smiles>CC(=O)C(C)(N)C(C)C=Cc1ccc2ccccc2c1</smiles>

$(2 S, 3 R)-3 \mathbf{j a}$

(2S,3R,E)-methyl 2-amino-2,3-dimethyl-5-(naphthalen-2-yl)pent-4-enoate

Prepared according to General Procedure A using Pd-6 and $(S, S p)$-L2-Cu, Purification by flash chromatography $(\mathrm{PE} / \mathrm{EA}=5: 1$ to $1: 1)$ afforded the product as a pale yellow solid, $41 \mathrm{mg}, 73 \%$ yield, m.p. $103-104^{\circ} \mathrm{C},>20: 1 \mathrm{dr},>99 \%$ ee, $\mathrm{R}_{\mathrm{f}}=0.3(\mathrm{PE} / \mathrm{EA}, 1: 1)$.

$[\alpha]^{25}{ }_{\mathrm{D}}=78.8\left(\mathrm{c} 0.5, \mathrm{CHCl}_{3}\right)$

${ }^{1} \mathbf{H}$ NMR (400 MHz, $\left.\mathbf{C D C l}_{3}\right) \delta 7.77(\mathrm{t}, J=7.5 \mathrm{~Hz}, 3 \mathrm{H}), 7.70(\mathrm{~s}, 1 \mathrm{H}), 7.59(\mathrm{dd}, J=8.6,1.5 \mathrm{~Hz}, 1 \mathrm{H})$, 7.43 (td, $J=7.1,1.5 \mathrm{~Hz}, 2 \mathrm{H}), 6.60(\mathrm{~d}, J=15.8 \mathrm{~Hz}, 1 \mathrm{H}), 6.30(\mathrm{dd}, J=15.8,9.1 \mathrm{~Hz}, 1 \mathrm{H}), 3.73(\mathrm{~s}, 3 \mathrm{H})$, 2.74-2.66(m, 1H), 1.65 (s, 2H), 1.34 (s, 3H), 1.09 (d, $J=6.9 \mathrm{~Hz}, 3 \mathrm{H})$.

${ }^{13}$ C NMR (101 MHz, CDCl 3 ) $\delta$ 177.88, 134.77, 133.64, 132.86, 131.97, 131.07, 128.13, 127.90, 127.66, 126.23, 125.86, 125.70, 123.69, 60.78, 52.23, 46.03, 24.91, 15.81 .

HRMS (ESI) calcd. for $\mathrm{C}_{18} \mathrm{H}_{22} \mathrm{NO}_{2}{ }^{+}(\mathrm{M}+\mathrm{H})^{+}:$: 284.1651, Found: 284.1644

the ee value $\mathrm{was}>99 \%, \mathrm{t}_{\mathrm{r}}($ minor $)=14.895 \mathrm{~min}, \mathrm{t}_{\mathrm{r}}$ (major) $=16.092 \mathrm{~min}($ Chiralcel $\mathrm{IC}, \lambda=220 \mathrm{~nm}$, hexanes $:{ }^{i} \mathrm{PrOH}=95: 5$, flow rate $=1.0 \mathrm{~mL} / \mathrm{min}$ ).

$\mathrm{mV}$

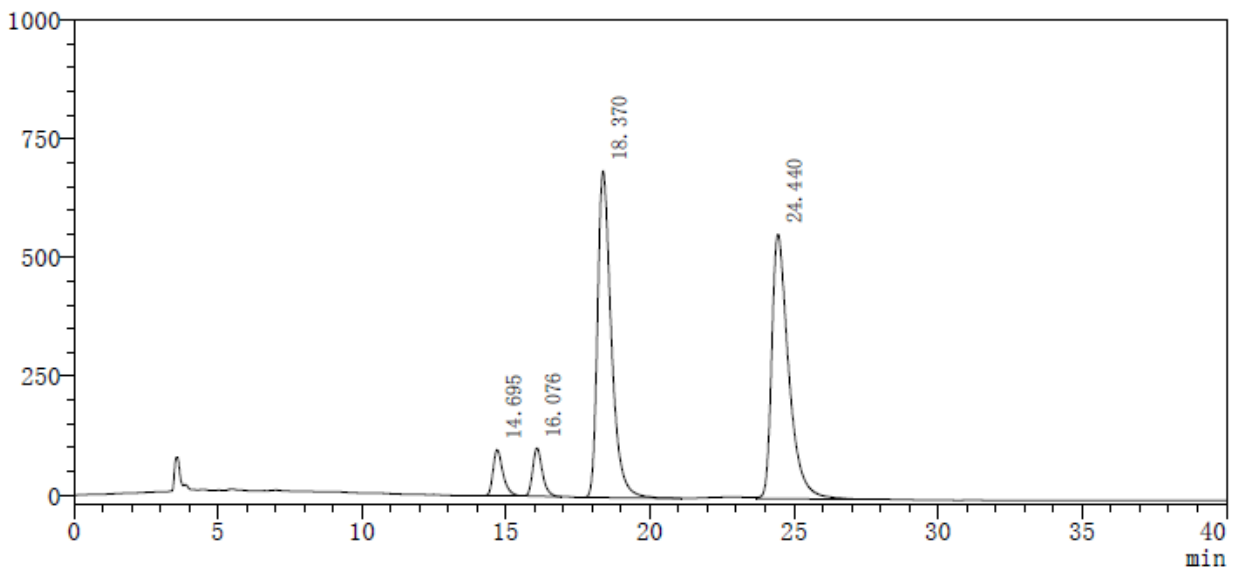

检测器A $220 \mathrm{~nm}$
\begin{tabular}{|c|c|c|c|c|c|}
\hline Peak\# & Time & Area & Height & Height\% & Area\% \\
\hline 1 & 14.695 & 2329077 & 96959 & 6.720 & 4.619 \\
\hline 2 & 16.076 & 2355617 & 101243 & 7.017 & 4.671 \\
\hline 3 & 18.370 & 22900041 & 687547 & 47.653 & 45.413 \\
\hline 4 & 24.440 & 22841178 & 557075 & 38.610 & 45.297 \\
\hline 总计 & & 50425913 & 1442824 & 100.000 & 100.000 \\
\hline
\end{tabular}


$\mathrm{mV}$

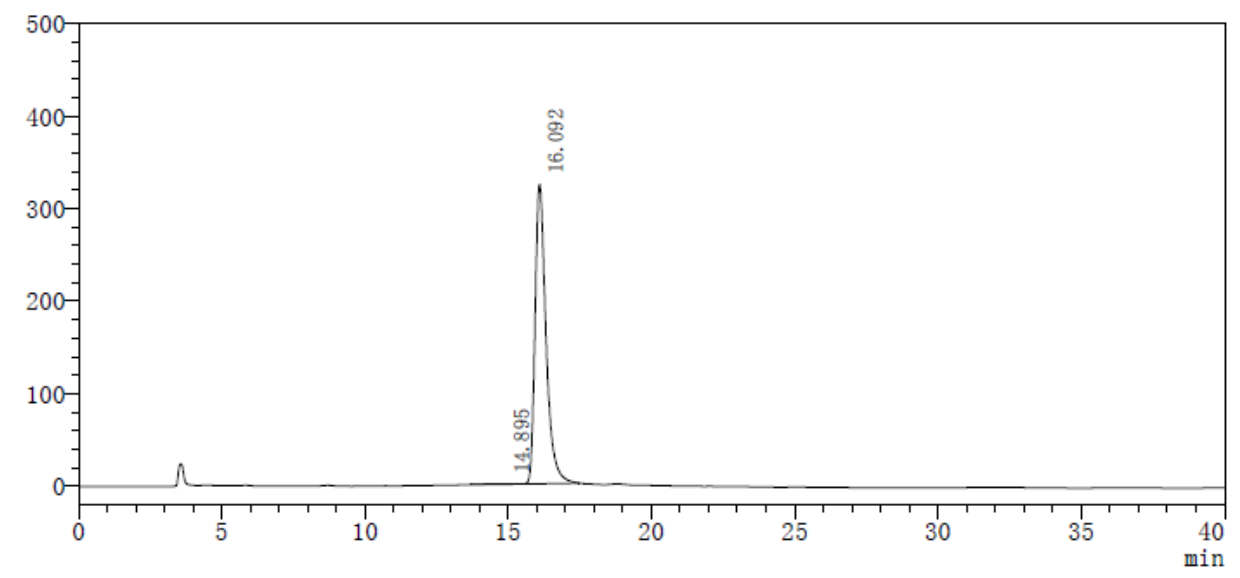

检测器A $220 \mathrm{~nm}$
\begin{tabular}{|c|c|c|c|c|c|}
\hline Peak\# & Time & Area & Height & Height\% & Area\% \\
\hline 1 & 14.895 & 20800 & 313 & 0.097 & 0.243 \\
\hline 2 & 16.092 & 8543185 & 323017 & 99.903 & 99.757 \\
\hline 总计 & & 8563985 & 323330 & 100.000 & 100.000 \\
\hline
\end{tabular}<smiles>CC(=O)[C@](C)(N)[C@@H](C)/C=C/c1ccc2ccccc2c1</smiles>

$(2 R, 3 R)-3 \mathbf{j a}$

\section{(2R,3R,E)-methyl 2-amino-2,3-dimethyl-5-(naphthalen-2-yl)pent-4-enoate}

Prepared according to General Procedure A using Pd-6 and $(R, R p)-\mathbf{L 2}-\mathbf{C u}$, Purification by flash chromatography (PE/EA $=5: 1$ to 1:1) afforded the product as a white solid, $39.5 \mathrm{mg}, 70 \%$ yield, m.p.62-64 ${ }^{\circ} \mathrm{C}, 14: 1 \mathrm{dr},>99 \%$ ee, $\mathrm{R}_{\mathrm{f}}=0.25$ (PE/EA, 1:1).

$[\alpha]^{25}=47.9\left(\mathrm{c} 0.5, \mathrm{CHCl}_{3}\right)$

${ }^{1} \mathbf{H}$ NMR (400 MHz, CDCl $) \delta 7.77(\mathrm{t}, J=8.7 \mathrm{~Hz}, 3 \mathrm{H}), 7.68(\mathrm{~s}, 1 \mathrm{H}), 7.56(\mathrm{dd}, J=8.5,1.6 \mathrm{~Hz}, 1 \mathrm{H})$, $7.52-7.37(\mathrm{~m}, 2 \mathrm{H}), 6.57(\mathrm{~d}, J=15.8 \mathrm{~Hz}, 1 \mathrm{H}), 6.25(\mathrm{dd}, J=15.8,8.5 \mathrm{~Hz}, 1 \mathrm{H}), 3.72(\mathrm{~s}, 3 \mathrm{H}), 2.93-$ $2.55(\mathrm{~m}, 1 \mathrm{H}), 1.67(\mathrm{~s}, 2 \mathrm{H}), 1.35(\mathrm{~s}, 3 \mathrm{H}), 1.16(\mathrm{~d}, J=6.9 \mathrm{~Hz}, 3 \mathrm{H})$.

${ }^{13}$ C NMR (101 MHz, CDCl 3$) \delta 177.50,134.71,133.61,132.87,131.59,131.28,128.14,127.89$, 127.66, 126.24, 125.93, 125.73, 123.60, 61.08, 52.18, 45.79, 23.56, 14.44.

HRMS (ESI) calcd. for $\mathrm{C}_{18} \mathrm{H}_{22} \mathrm{NO}_{2}{ }^{+}(\mathrm{M}+\mathrm{H})^{+}:$284.1651, Found: 284.1642

the ee value $\mathrm{was}>99 \%, \mathrm{t}_{\mathrm{r}}($ minor $)=18.446 \mathrm{~min}, \mathrm{t}_{\mathrm{r}}$ (major) $=24.490 \mathrm{~min}$ (Chiralcel IC, $\lambda=220 \mathrm{~nm}$, hexanes $:{ }^{i} \mathrm{PrOH}=95: 5$, flow rate $\left.=1.0 \mathrm{~mL} / \mathrm{min}\right)$. 
$\mathrm{mV}$

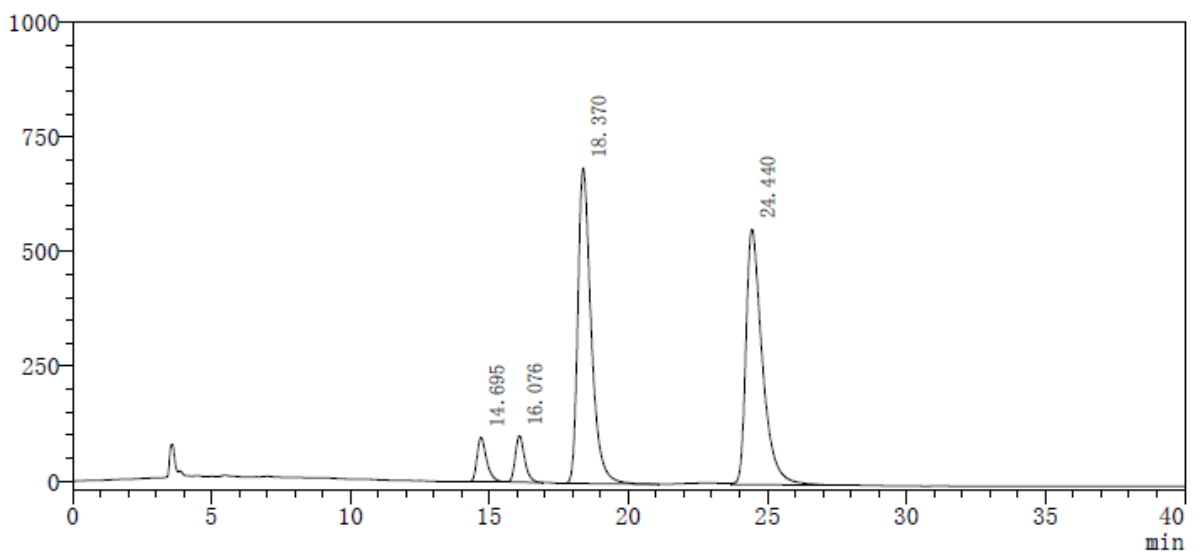

检测器A $220 \mathrm{~nm}$
\begin{tabular}{|c|c|c|c|c|c|}
\hline Peak\# & Time & Area & Height & Height\% & Area\% \\
\hline 1 & 14.695 & 2329077 & 96959 & 6.720 & 4.619 \\
\hline 2 & 16.076 & 2355617 & 101243 & 7.017 & 4.671 \\
\hline 3 & 18.370 & 22900041 & 687547 & 47.653 & 45.413 \\
\hline 4 & 24.440 & 22841178 & 557075 & 38.610 & 45.297 \\
\hline 总计 & & 50425913 & 1442824 & 100.000 & 100.000 \\
\hline
\end{tabular}

$\mathrm{mV}$

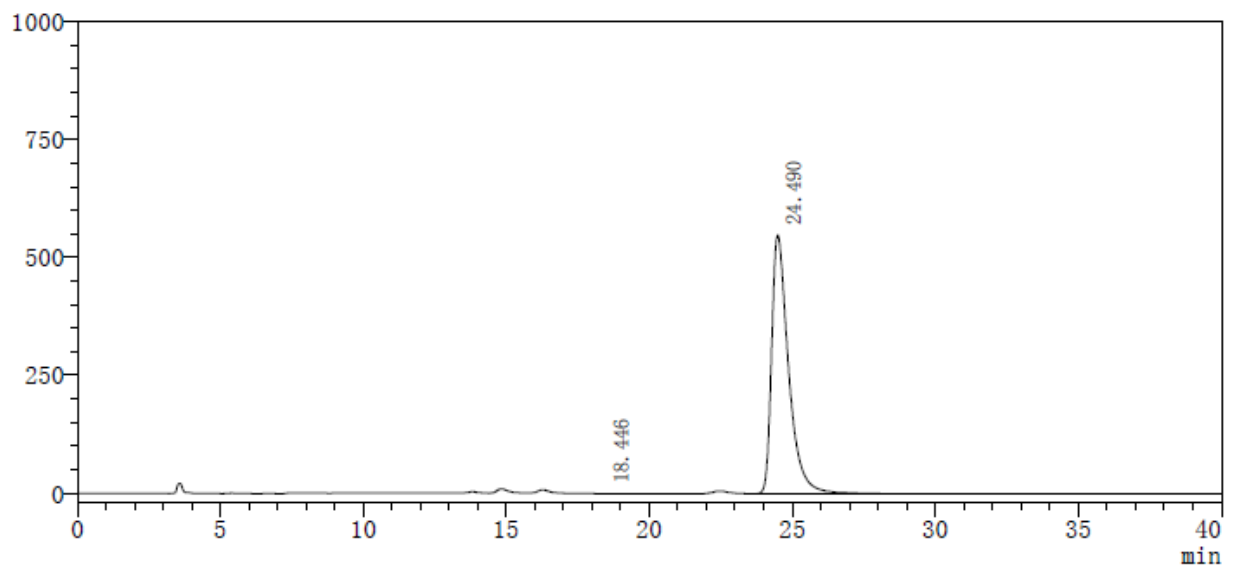

检测器A $220 \mathrm{~nm}$
\begin{tabular}{|c|c|c|c|c|c|}
\hline Peak\# & Time & Area & Height & Height\% & Area\% \\
\hline 1 & 18.446 & 4978 & 58 & 0.011 & 0.022 \\
\hline 2 & 24.490 & 22547708 & 548299 & 99.989 & 99.978 \\
\hline 总计 & & 22552686 & 548357 & 100.000 & 100.000 \\
\hline
\end{tabular}<smiles>CC(=O)C(C)(N)C([Mg])C=Cc1ccco1</smiles>

$(2 S, 3 R)-3 \mathbf{k a}$

(2S,3R,E)-methyl 2-amino-5-(furan-2-yl)-2,3-dimethylpent-4-enoate

Prepared according to General Procedure A using Pd-6 and $(S, S p)$-L2-Cu, Purification by flash chromatography $(\mathrm{PE} / \mathrm{EA}=5: 1$ to $1: 1)$ afforded the product as a pale yellow oil, $31 \mathrm{mg}, 69 \%$ 
yield, $>20: 1 \mathrm{dr},>99 \%$ ee, $\mathrm{R}_{\mathrm{f}}=0.3(\mathrm{PE} / \mathrm{EA}, 1: 1)$.

$[\alpha]^{25} \mathrm{D}=46.2\left(\mathrm{c} 1.0, \mathrm{CHCl}_{3}\right)$

${ }^{1}$ H NMR (400 MHz, CDCl 3 ) $\delta 7.32(\mathrm{~s}, 1 \mathrm{H}), 6.35(\mathrm{dd}, J=3.1,1.8 \mathrm{~Hz}, 1 \mathrm{H}), 6.26(\mathrm{~d}, J=15.9 \mathrm{~Hz}, 1 \mathrm{H})$, $6.18(\mathrm{~d}, J=3.1 \mathrm{~Hz}, 1 \mathrm{H}), 6.11(\mathrm{dd}, J=15.9,9.0 \mathrm{~Hz}, 1 \mathrm{H}), 3.73(\mathrm{~s}, 3 \mathrm{H}), 2.77-2.47(\mathrm{~m}, 1 \mathrm{H}), 1.63(\mathrm{~s}$, $2 \mathrm{H}), 1.31(\mathrm{~s}, 3 \mathrm{H}), 1.03(\mathrm{~d}, J=6.9 \mathrm{~Hz}, 3 \mathrm{H})$.

${ }^{13}$ C NMR (101 MHz, CDCl $) \delta 177.65,152.77,141.57,129.35,120.34,111.16,106.96,60.68,52.16$, 45.60, 24.90, 15.55 .

HRMS (ESI) calcd. for $\mathrm{C}_{12} \mathrm{H}_{18} \mathrm{NO}_{3}{ }^{+}(\mathrm{M}+\mathrm{H})^{+}:$:224.1287, Found: 224.1280

the ee value was $>99 \%, \mathrm{t}_{\mathrm{r}}($ minor $)=26.438 \mathrm{~min}, \mathrm{t}_{\mathrm{r}}($ major $)=31.155 \mathrm{~min}$ (Chiralcel $\mathrm{IC}, \lambda=220 \mathrm{~nm}$, hexanes $:{ }^{i} \mathrm{PrOH}=98: 2$, flow rate $=1.0 \mathrm{~mL} / \mathrm{min}$ ).

$\mathrm{mV}$

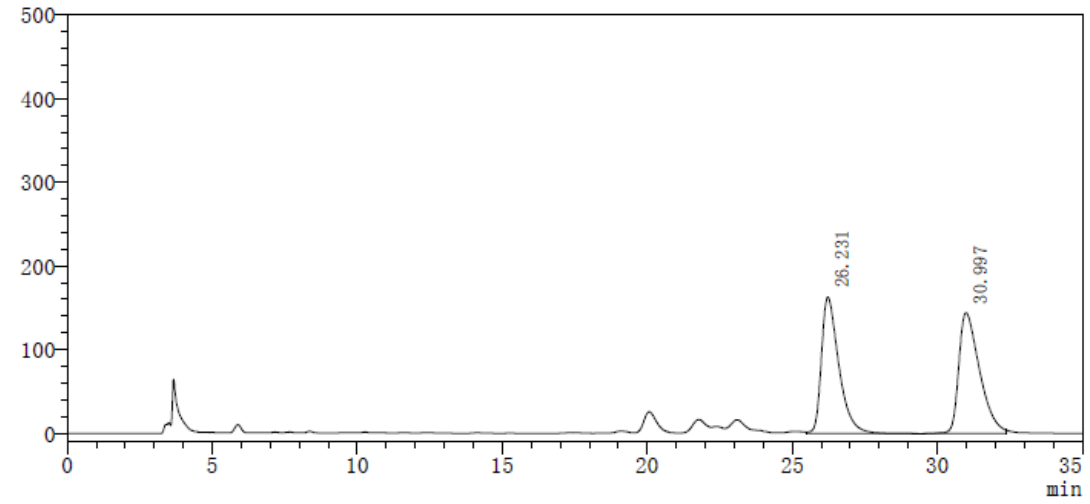

检测器A $220 \mathrm{~nm}$
\begin{tabular}{|c|c|c|c|c|c|}
\hline Peak\# & Time & Area & Height & Height\% & Area\% \\
\hline 1 & 26.231 & 6826862 & 162869 & 53.024 & 48.710 \\
\hline 2 & 30.997 & 7188432 & 144291 & 46.976 & 51.290 \\
\hline 总计 & & 14015294 & 307160 & 100.000 & 100.000 \\
\hline
\end{tabular}

$\mathrm{mV}$

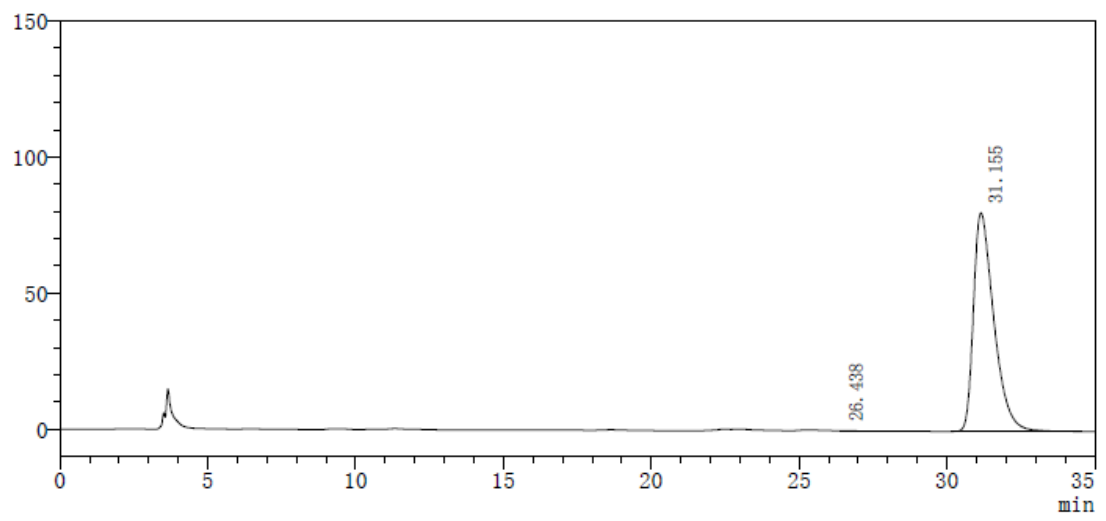

检测器A $220 \mathrm{~nm}$
\begin{tabular}{|c|c|c|c|c|c|}
\hline Peak\# & Time & Area & Height & Height\% & Area\% \\
\hline 1 & 26.438 & 5973 & 120 & 0.149 & 0.154 \\
\hline 2 & 31.155 & 3867000 & 80340 & 99.851 & 99.846 \\
\hline 总计 & & 3872973 & 80460 & 100.000 & 100.000 \\
\hline
\end{tabular}




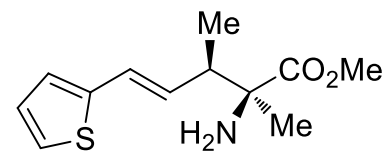

(2S, 3R)-3la

\section{(2S,3R,E)-methyl 2-amino-2,3-dimethyl-5-(thiophen-2-yl)pent-4-enoate}

Prepared according to General Procedure A using Pd-6 and $(S, S p)$-L2-Cu, Purification by flash chromatography $(\mathrm{PE} / \mathrm{EA}=5: 1$ to 1:1) afforded the product as a pale yellow oil, $32 \mathrm{mg}, 67 \%$ yield, >20:1 dr, >99\% ee, $\mathrm{R}_{\mathrm{f}}=0.3(\mathrm{PE} / \mathrm{EA}, 1: 1)$.

$[\alpha]^{25}=87.9\left(\mathrm{c} 0.5, \mathrm{CHCl}_{3}\right)$

${ }^{1} \mathbf{H}$ NMR $\left(400 \mathrm{MHz}, \mathbf{C D C l}_{3}\right) \delta 7.11(\mathrm{~d}, J=4.9 \mathrm{~Hz}, 1 \mathrm{H}), 6.93(\mathrm{dt}, J=9.0,3.3 \mathrm{~Hz}, 2 \mathrm{H}), 6.57(\mathrm{~d}, J=$ $15.7 \mathrm{~Hz}, 1 \mathrm{H}), 6.00(\mathrm{dd}, J=15.7,9.1 \mathrm{~Hz}, 1 \mathrm{H}), 3.73(\mathrm{~s}, 3 \mathrm{H}), 2.66-2.55(\mathrm{~m}, 1 \mathrm{H}), 1.67(\mathrm{~s}, 2 \mathrm{H}), 1.31$ (s, $3 \mathrm{H}), 1.03(\mathrm{~d}, J=6.9 \mathrm{~Hz}, 3 \mathrm{H})$.

${ }^{13}$ C NMR (101 MHz, CDCl 3$) \delta 177.70,142.49,130.34,127.26,124.98,123.71,60.68,52.20,45.78$, $24.88,15.60$

HRMS (ESI) calcd. for $\mathrm{C}_{12} \mathrm{H}_{18} \mathrm{NO}_{2} \mathrm{~S}^{+}(\mathrm{M}+\mathrm{H})^{+}: 240.1058$, Found: 240.1052

the ee value was $>99 \%, \mathrm{t}_{\mathrm{r}}($ minor $)=21.743 \mathrm{~min}, \mathrm{t}_{\mathrm{r}}($ major $)=31.803 \mathrm{~min}($ Chiralcel $\mathrm{IC}, \lambda=220 \mathrm{~nm}$, hexanes $:{ }^{i} \mathrm{PrOH}=98: 2$, flow rate $\left.=1.0 \mathrm{~mL} / \mathrm{min}\right)$.

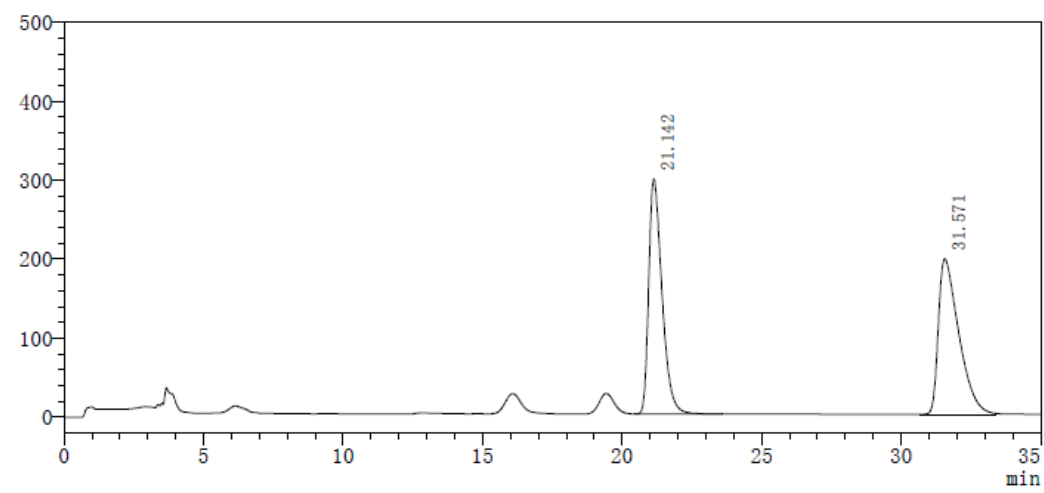

检测器A $220 \mathrm{~nm}$
\begin{tabular}{|c|c|c|c|c|c|}
\hline Peakk & Time & Area & Height & Height $\%$ & Area\% \\
\hline 1 & 21.142 & 9841538 & 297509 & 60.176 & 49.513 \\
\hline 2 & 31.571 & 10035173 & 196891 & 39.824 & 50.487 \\
\hline 总计 & & 19876712 & 494401 & 100.000 & 100.000 \\
\hline
\end{tabular}




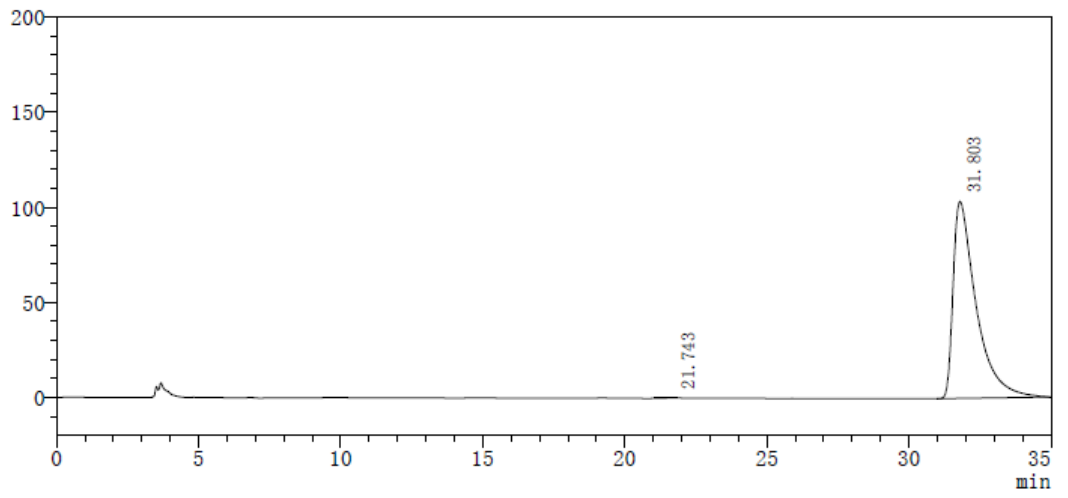

检测器A $220 \mathrm{~nm}$
\begin{tabular}{|c|c|c|c|c|c|}
\hline Peak\# & Time & Area & Height & Height $\%$ & Area\% \\
\hline 1 & 21.743 & 5110 & 191 & 0.184 & 0.087 \\
\hline 2 & 31.803 & 5836285 & 103519 & 99.816 & 99.913 \\
\hline 总计 & & 5841395 & 103709 & 100.000 & 100.000 \\
\hline
\end{tabular}<smiles>COC(=O)C(C)(N)/C=C/c1c[n-]c2ccccc12</smiles>

$(2 S, 3 R)-3 m a$

\section{(2S,3R,E)-methyl 2-amino-2,3-dimethyl-5-(1-tosyl-1H-indol-3-yl)pent-4-enoate}

Prepared according to General Procedure A using Pd-6 and $(S, S p)$-L2-Cu, Purification by flash chromatography $(\mathrm{PE} / \mathrm{EA}=5: 1$ to $1: 2)$ afforded the product as a pale yellow oil, $81 \mathrm{mg}, 96 \%$ yield, $>20: 1 \mathrm{dr},>99 \%$ ee, $\mathrm{R}_{\mathrm{f}}=0.2$ (PE/EA, 1:1).

$[\alpha]^{25} \mathrm{D}=37.5\left(\mathrm{c} 1.0, \mathrm{CHCl}_{3}\right)$

${ }^{1}$ H NMR (400 MHz, CDCl 3 ) $\delta 7.99(\mathrm{~d}, J=8.2 \mathrm{~Hz}, 1 \mathrm{H}), 7.77(\mathrm{~d}, J=8.4 \mathrm{~Hz}, 2 \mathrm{H}), 7.71(\mathrm{~d}, J=7.6 \mathrm{~Hz}$, 1H), $7.56(\mathrm{~s}, 1 \mathrm{H}), 7.36-7.29(\mathrm{~m}, 1 \mathrm{H}), 7.29-7.24(\mathrm{~m}, 1 \mathrm{H}), 7.21(\mathrm{~d}, J=8.1 \mathrm{~Hz}, 2 \mathrm{H}), 6.50(\mathrm{~d}, J=16.1$ $\mathrm{Hz}, 1 \mathrm{H}), 6.22(\mathrm{dd}, J=16.1,9.1 \mathrm{~Hz}, 1 \mathrm{H}), 3.74(\mathrm{~s}, 3 \mathrm{H}), 2.69-2.59(\mathrm{~m}, 1 \mathrm{H}), 2.32(\mathrm{~s}, 3 \mathrm{H}), 1.67(\mathrm{~s}, 2 \mathrm{H})$, $1.33(\mathrm{~s}, 3 \mathrm{H}), 1.07(\mathrm{~d}, J=6.9 \mathrm{~Hz}, 3 \mathrm{H})$.

${ }^{13}$ C NMR (101 MHz, CDCl 3$) \delta 177.81,144.98,135.43,135.17,132.29,129.91,129.13,126.87$, $124.87,123.43,123.30,122.15,120.48,120.38,113.71,60.65,52.22,46.49,24.81,21.57,15.77$. HRMS (ESI) calcd. for $\mathrm{C}_{23} \mathrm{H}_{27} \mathrm{~N}_{2} \mathrm{O}_{4} \mathrm{~S}^{+}(\mathrm{M}+\mathrm{H})^{+}:$427.1692, Found: 427.1679

the ee value was $>99 \%, \mathrm{t}_{\mathrm{r}}($ major $)=10.992 \mathrm{~min}, \mathrm{t}_{\mathrm{r}}($ minor $)=13.088 \mathrm{~min}$ (Chiralcel $\mathrm{IC}, \lambda=220 \mathrm{~nm}$, hexanes $:{ }^{i} \mathrm{PrOH}=80: 20$, flow rate $=1.0 \mathrm{~mL} / \mathrm{min}$ ). 
$\mathrm{mV}$

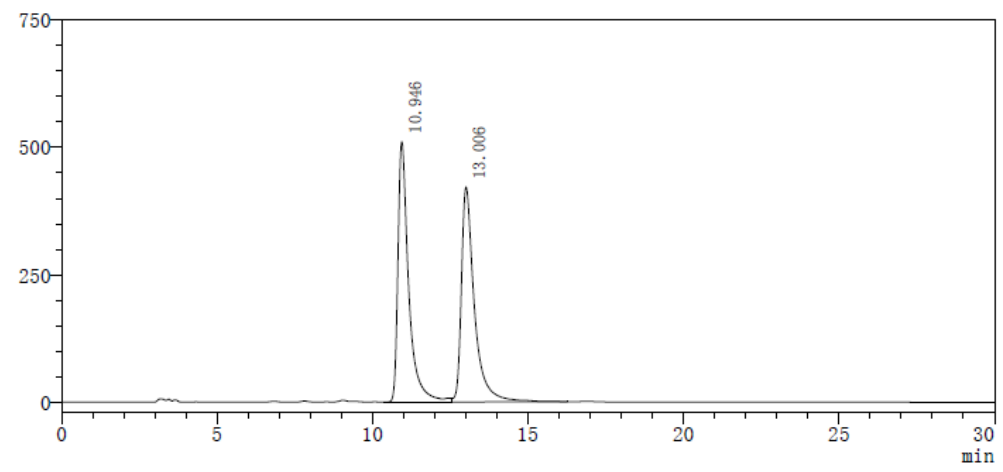

检测器A $220 \mathrm{~nm}$
\begin{tabular}{|c|c|c|c|c|c|}
\hline Peak\# & Time & Area & Height & Height $\%$ & Area \\
\hline 1 & 10.946 & 12287091 & 510825 & 54.808 & 50.107 \\
\hline 2 & 13.006 & 12234677 & 421203 & 45.192 & 49.893 \\
\hline 总计 & & 24521767 & 932028 & 100.000 & 100.000 \\
\hline
\end{tabular}

$\mathrm{mV}$

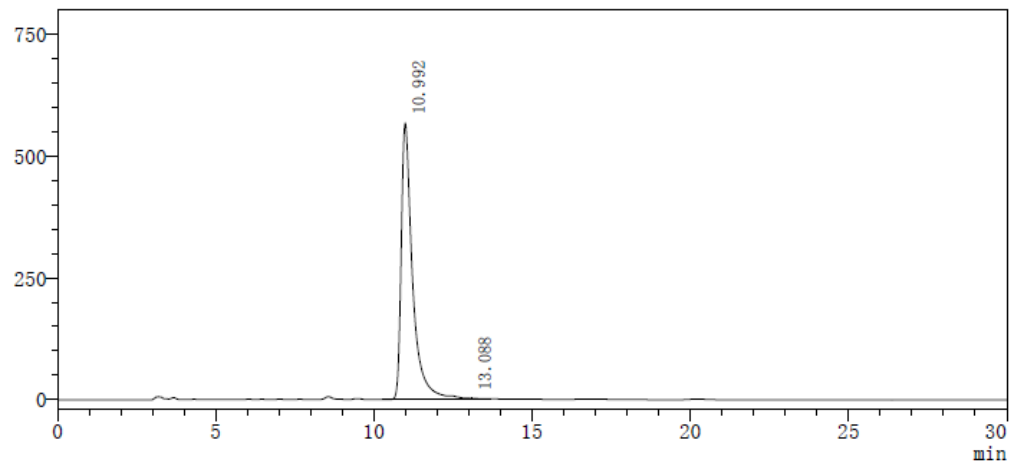

检测器A $220 \mathrm{~nm}$
\begin{tabular}{|c|c|c|c|c|c|}
\hline Peak\# & Time & Area & Height & Height\% & Area\% \\
\hline 1 & 10.992 & 14226262 & 567064 & 99.545 & 99.513 \\
\hline 2 & 13.088 & 69643 & 2592 & 0.455 & 0.487 \\
\hline 总计 & & 14295905 & 569656 & 100.000 & 100.000 \\
\hline
\end{tabular}<smiles>[M]C(/C=C/CCOC(C)=O)C(C)(N)C(C)=O</smiles>

$(2 S, 3 R)-3 n a$

(2S,3R,E)-methyl 7-acetoxy-2-amino-2,3-dimethylhept-4-enoate

Prepared according to General Procedure B using Pd-6 and $(S, S p)$-L2-Cu, Purification by flash chromatography $(\mathrm{PE} / \mathrm{EA}=5: 1$ to $1: 2)$ afforded the product as a pale yellow oil, $22 \mathrm{mg}, 46 \%$ yield, $>20: 1 \mathrm{dr}, 93 \%$ ee, $\mathrm{R}_{\mathrm{f}}=0.2(\mathrm{PE} / \mathrm{EA}, 1: 1)$.

$[\alpha]^{25} \mathrm{D}=48.8\left(\mathrm{c} 0.2, \mathrm{CHCl}_{3}\right)$

${ }^{1}$ H NMR (400 MHz, CDCl $) \delta 5.48-5.43(\mathrm{~m}, 2 \mathrm{H}), 4.06(\mathrm{td}, J=6.7,1.6 \mathrm{~Hz}, 2 \mathrm{H}), 3.70(\mathrm{~s}, 3 \mathrm{H}), 2.56-$ $2.44(\mathrm{~m}, 1 \mathrm{H}), 2.40-2.28(\mathrm{~m}, 2 \mathrm{H}), 2.05(\mathrm{~s}, 3 \mathrm{H}), 1.68(\mathrm{~s}, 2 \mathrm{H}), 1.26(\mathrm{~s}, 3 \mathrm{H}), 1.01(\mathrm{~d}, J=6.9 \mathrm{~Hz}, 3 \mathrm{H})$.

${ }^{13}$ C NMR (101 MHz, $\left.\mathbf{C D C l}_{3}\right) \delta 177.54,171.09,133.71,127.45,63.75,60.68,51.98,44.96,32.01$, 23.36, 20.92, 14.14.

HRMS (ESI) calcd. for $\mathrm{C}_{12} \mathrm{H}_{22} \mathrm{NO}_{4}{ }^{+}(\mathrm{M}+\mathrm{H})^{+}: 244.1549$, Found: 244.1543

the ee value $\mathrm{was} 93 \%, \mathrm{t}_{\mathrm{r}}$ (major) $=23.699 \mathrm{~min}, \mathrm{t}_{\mathrm{r}}($ minor $)=32.204 \mathrm{~min}($ Chiralcel $\mathrm{IE}, \lambda=204 \mathrm{~nm}$, 
hexanes $:{ }^{i} \mathrm{PrOH}=90: 10$, flow rate $\left.=1.0 \mathrm{~mL} / \mathrm{min}\right)$.

$\mathrm{mV}$
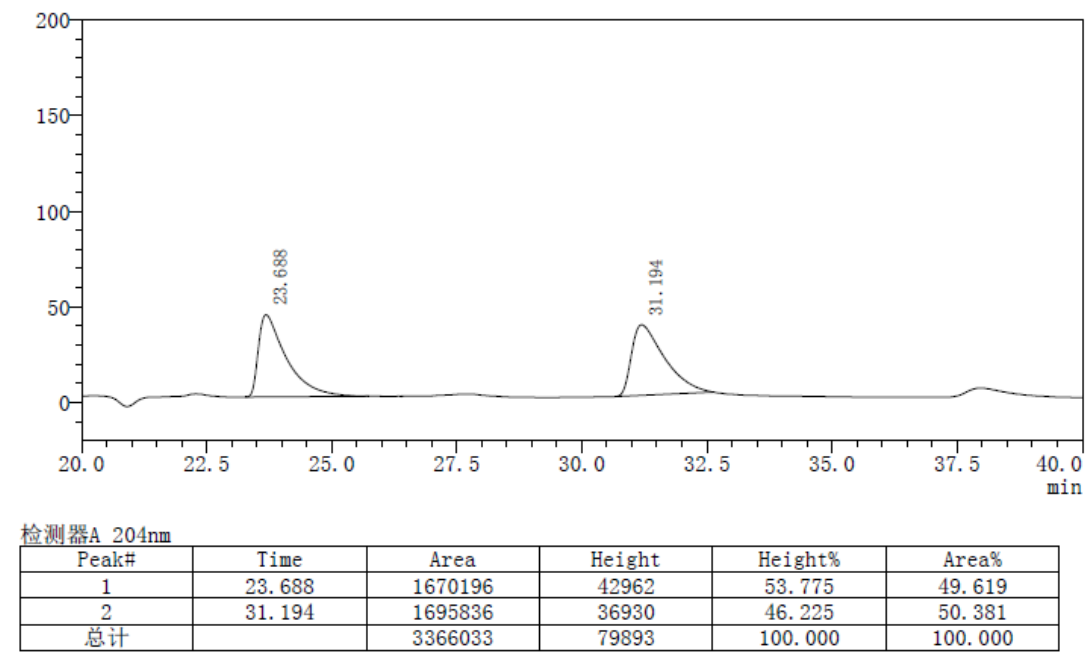

$\mathrm{mV}$

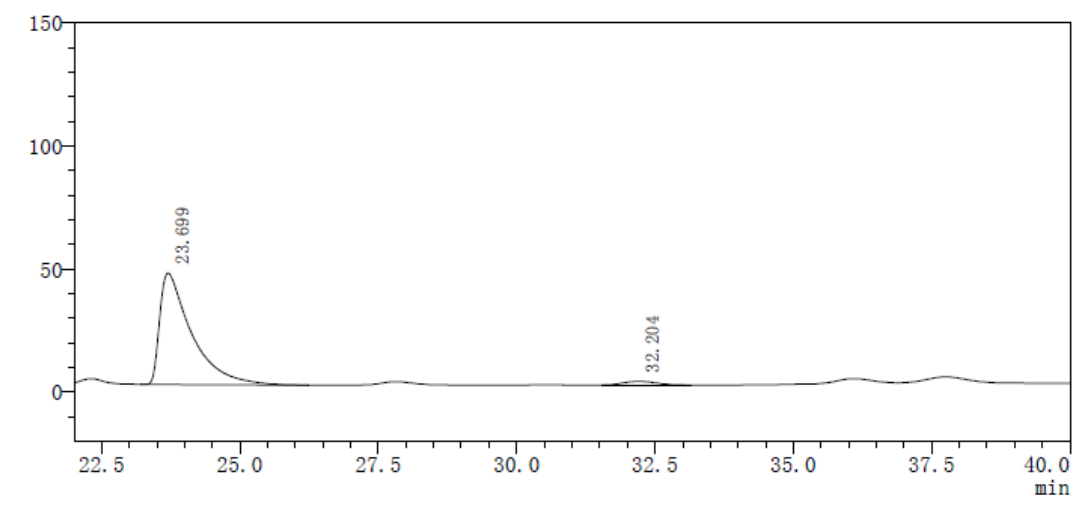

检测器A $204 \mathrm{~nm}$
\begin{tabular}{|c|c|c|c|c|c|}
\hline Peak\# & Time & Area & Height & Height $\%$ & Area\% \\
\hline 1 & 23.699 & 1820559 & 45238 & 96.658 & 96.569 \\
\hline 2 & 32.204 & 64691 & 1564 & 3.342 & 3.431 \\
\hline 总计 & & 1885251 & 46802 & 100.000 & 100.000 \\
\hline
\end{tabular}<smiles>CC[C@@H](/C=C/c1ccccc1)[C@@](C)(N)C(C)=O</smiles>

$(2 S, 3 R)-30 a$

\section{(2S,3R,E)-methyl 2-amino-3-ethyl-2-methyl-5-phenylpent-4-enoate}

Prepared according to General Procedure B using Pd-6 and $(S, S p)$-L2-Cu, Purification by flash chromatography (PE/EA = 5:1 to 1:1) afforded the product as a pale yellow oil, $27.5 \mathrm{mg}, 56 \%$ yield, 5:1 dr, $>99 \%$ ee, $\mathrm{R}_{\mathrm{f}}=0.4$ (PE/EA, 1:1).

$[\alpha]^{25}=56.7\left(\mathrm{c} 0.5, \mathrm{CHCl}_{3}\right)$

${ }^{1} \mathbf{H}$ NMR (400 MHz, CDCl $) \delta 7.39(\mathrm{~d}, J=7.3 \mathrm{~Hz}, 2 \mathrm{H}), 7.30(\mathrm{t}, J=7.5 \mathrm{~Hz}, 2 \mathrm{H}), 7.22(\mathrm{~d}, J=7.3 \mathrm{~Hz}$, $1 \mathrm{H}), 6.44(\mathrm{~d}, J=15.8 \mathrm{~Hz}, 1 \mathrm{H}), 6.02(\mathrm{dd}, J=15.8,9.8 \mathrm{~Hz}, 1 \mathrm{H}), 3.72(\mathrm{~s}, 3 \mathrm{H}), 2.37-2.25(\mathrm{~m}, 1 \mathrm{H}), 1.65(\mathrm{~s}$, $2 \mathrm{H}), 1.50-1.41(\mathrm{~m}, 1 \mathrm{H}), 1.39-1.31(\mathrm{~m}, 1 \mathrm{H}), 1.29(\mathrm{~s}, 3 \mathrm{H}), 0.87(\mathrm{t}, J=7.4 \mathrm{~Hz}, 3 \mathrm{H})$. 
${ }^{13} \mathbf{C}$ NMR (101 MHz, $\left.\mathbf{C D C l}_{3}\right) \delta 178.14,137.30,133.72,129.08,128.52,127.24,126.25,60.93,54.07$, $52.18,25.01,22.64,12.58$.

HRMS (ESI) calcd. for $\mathrm{C}_{15} \mathrm{H}_{22} \mathrm{NO}_{2}{ }^{+}(\mathrm{M}+\mathrm{H})^{+}:$248.1651, Found: 248.1646

the ee value $\mathrm{was}>99 \%, \mathrm{t}_{\mathrm{r}}($ minor $)=14.729 \mathrm{~min}, \mathrm{t}_{\mathrm{r}}($ major $)=21.198 \mathrm{~min}($ Chiralcel $\mathrm{IC}, \lambda=220 \mathrm{~nm}$, hexanes $:{ }^{i} \mathrm{PrOH}=98: 2$, flow rate $=1.0 \mathrm{~mL} / \mathrm{min}$ ).

$\mathrm{mV}$

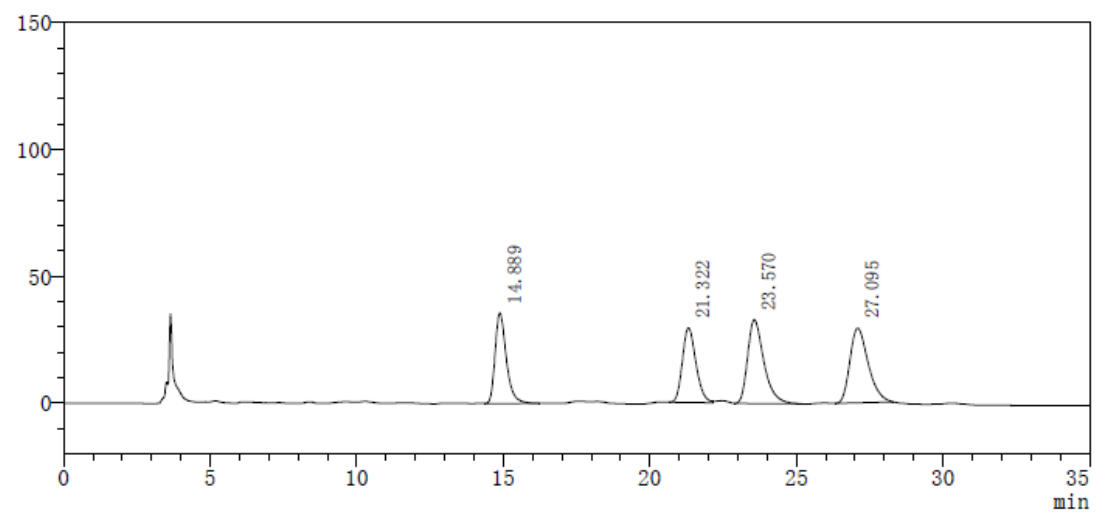

检测器A $220 \mathrm{~nm}$
\begin{tabular}{|c|c|c|c|c|c|}
\hline Peak\# & Time & Area & Height & Height\% & Area\% \\
\hline 1 & 14.889 & 949586 & 35671 & 27.980 & 21.306 \\
\hline 2 & 21.322 & 950479 & 29410 & 23.069 & 21.326 \\
\hline 3 & 23.570 & 1275602 & 32954 & 25.849 & 28.621 \\
\hline 4 & 27.095 & 1281202 & 29452 & 23.102 & 28.747 \\
\hline 总计 & & 4456868 & 127487 & 100.000 & 100.000 \\
\hline
\end{tabular}

$\mathrm{mV}$

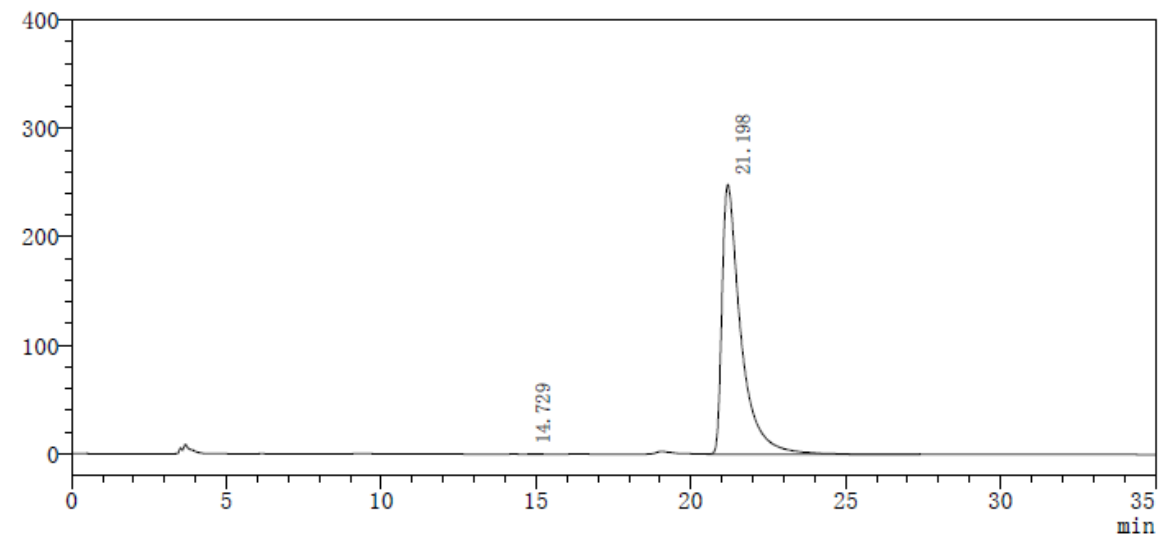

检测器A $220 \mathrm{~nm}$
\begin{tabular}{|c|c|c|c|c|c|}
\hline Peak\# & Time & Area & Height & Height\% & Area\% \\
\hline 1 & 14.729 & 2421 & 105 & 0.042 & 0.023 \\
\hline 2 & 21.198 & 10573106 & 248719 & 99.958 & 99.977 \\
\hline 总计 & & 10575527 & 248824 & 100.000 & 100.000 \\
\hline
\end{tabular}<smiles>CCC(/C=C/c1ccccc1)C(C)(N)C(C)=O</smiles>

$(2 R, 3 R)-30 a$ 
Prepared according to General Procedure B using Pd-6 and $(R, R p)-\mathbf{L 2}-\mathbf{C u}$, Purification by flash chromatography (PE/EA = 5:1 to 1:1) afforded the product as a pale yellow oil, $27 \mathrm{mg}, 56 \%$ yield, 5:1 $\mathrm{dr},>99 \%$ ee, $\mathrm{R}_{\mathrm{f}}=0.35$ (PE/EA, 1:1).

$[\alpha]^{25} \mathrm{D}=10.7\left(\mathrm{c} 0.5, \mathrm{CHCl}_{3}\right)$

${ }^{1} \mathbf{H}$ NMR $\left(400 \mathrm{MHz}, \mathbf{C D C l}_{3}\right) \delta 7.36(\mathrm{~d}, J=7.6 \mathrm{~Hz}, 2 \mathrm{H}), 7.30(\mathrm{t}, J=7.4 \mathrm{~Hz}, 2 \mathrm{H}), 7.22(\mathrm{t}, J=6.9 \mathrm{~Hz}$, $1 \mathrm{H}), 6.41(\mathrm{~d}, J=15.8 \mathrm{~Hz}, 1 \mathrm{H}), 5.97(\mathrm{dd}, J=15.7,9.8 \mathrm{~Hz}, 1 \mathrm{H}), 3.70(\mathrm{~s}, 3 \mathrm{H}), 2.36-2.24(\mathrm{~m}, 1 \mathrm{H}), 1.76$ $(\mathrm{s}, 2 \mathrm{H}), 1.67-1.54(\mathrm{~m}, 1 \mathrm{H}), 1.43-1.35(\mathrm{~m}, 1 \mathrm{H}), 1.33(\mathrm{~s}, 3 \mathrm{H}), 0.88(\mathrm{t}, J=7.2 \mathrm{~Hz}, 3 \mathrm{H})$.

${ }^{13} \mathbf{C}$ NMR (101 MHz, CDCl $) \delta 176.09,136.14,132.49,128.21,127.43,126.23,125.15,60.06,53.53$, $50.99,22.52,20.70,11.50$.

HRMS (ESI) calcd. for $\mathrm{C}_{15} \mathrm{H}_{22} \mathrm{NO}_{2}{ }^{+}(\mathrm{M}+\mathrm{H})^{+}:$:248.1651, Found: 248.1649

the ee value was $>99 \%, \mathrm{t}_{\mathrm{r}}($ major $)=23.681 \mathrm{~min}, \mathrm{t}_{\mathrm{r}}($ minor $)=23.731 \mathrm{~min}($ Chiralcel $\mathrm{IC}, \lambda=220 \mathrm{~nm}$, hexanes $:{ }^{i} \mathrm{PrOH}=98: 2$, flow rate $\left.=1.0 \mathrm{~mL} / \mathrm{min}\right)$.

$\mathrm{mV}$

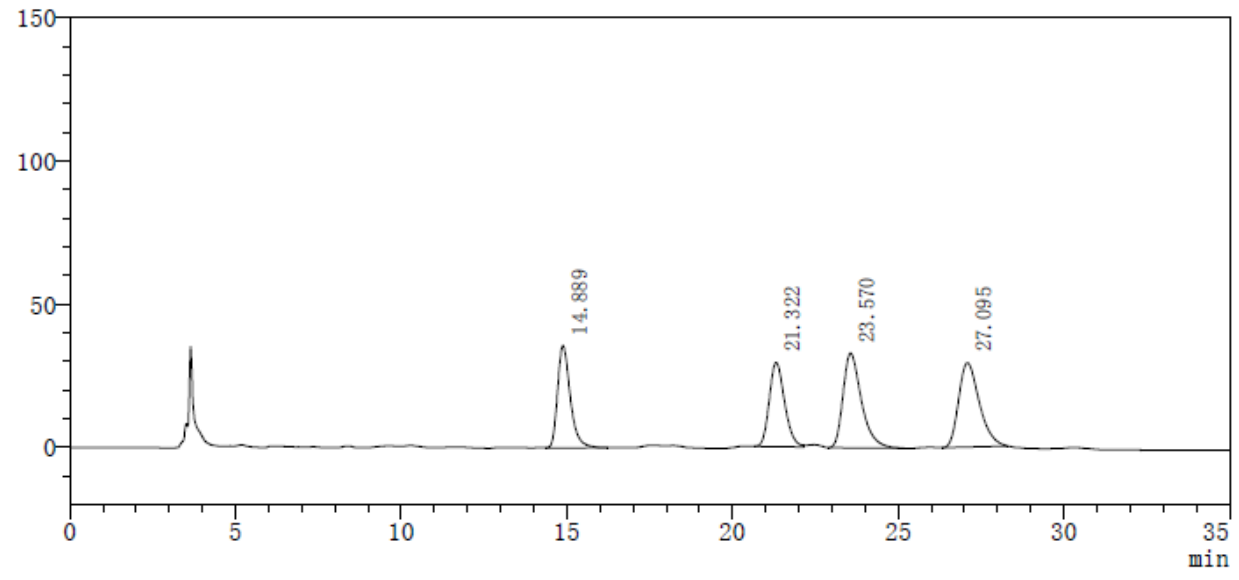

检测器A $220 \mathrm{~nm}$
\begin{tabular}{|c|c|c|c|c|c|}
\hline Peak\# & Time & Area & Height & Height\% & Area\% \\
\hline 1 & 14.889 & 949586 & 35671 & 27.980 & 21.306 \\
\hline 2 & 21.322 & 950479 & 29410 & 23.069 & 21.326 \\
\hline 3 & 23.570 & 1275602 & 32954 & 25.849 & 28.621 \\
\hline 4 & 27.095 & 1281202 & 29452 & 23.102 & 28.747 \\
\hline 总计 & & 4456868 & 127487 & 100.000 & 100.000 \\
\hline
\end{tabular}




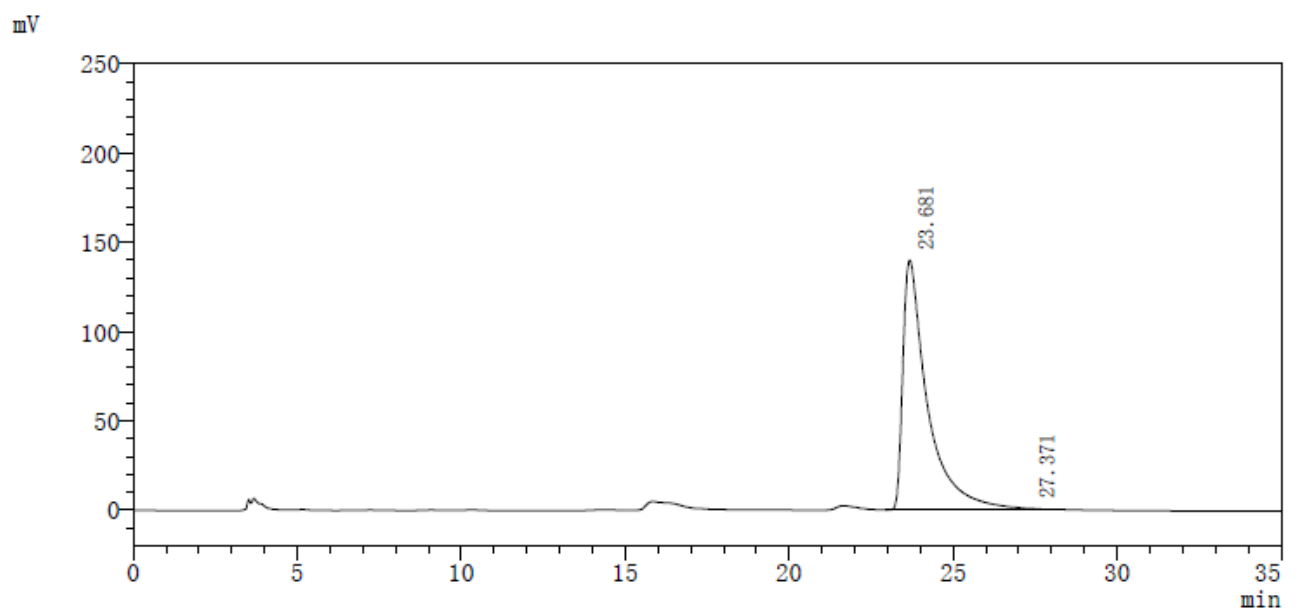

检测器A 220nm
\begin{tabular}{|c|c|c|c|c|c|}
\hline Peak\# & Time & Area & Height & Height\% & Area\% \\
\hline 1 & 23.681 & 7357909 & 139759 & 99.380 & 99.585 \\
\hline 2 & 27.371 & 30676 & 872 & 0.620 & 0.415 \\
\hline 总计 & & 7388585 & 140631 & 100.000 & 100.000 \\
\hline
\end{tabular}<smiles>CCCC(/C=C/c1ccccc1)C(C)(N)C(C)=O</smiles>

$(2 S, 3 R)-3 p a$

\section{(2S,3R)-methyl 2-amino-2-methyl-3-((E)-styryl)hexanoate}

Prepared according to General Procedure B using Pd-6 and $(S, S p)$-L2-Cu, Purification by flash chromatography (PE/EA = 5:1 to 1:1) afforded the product as a pale yellow oil, $24.5 \mathrm{mg}, 47 \%$ yield, $7: 1 \mathrm{dr},>99 \%$ ee, $\mathrm{R}_{\mathrm{f}}=0.4(\mathrm{PE} / \mathrm{EA}, 1: 1)$.

$[\alpha]^{25}=37.6\left(\mathrm{c} 0.2, \mathrm{CHCl}_{3}\right)$

${ }^{1} \mathbf{H}$ NMR (400 MHz, CDCl $) \delta 7.38(\mathrm{~d}, J=7.4 \mathrm{~Hz}, 2 \mathrm{H}), 7.30(\mathrm{t}, J=7.5 \mathrm{~Hz}, 2 \mathrm{H}), 7.21(\mathrm{t}, J=7.3 \mathrm{~Hz}$, $1 \mathrm{H}), 6.42(\mathrm{~d}, J=15.8 \mathrm{~Hz}, 1 \mathrm{H}), 6.04(\mathrm{dd}, J=15.8,9.8 \mathrm{~Hz}, 1 \mathrm{H}), 3.72(\mathrm{~s}, 3 \mathrm{H}), 2.57-2.34(\mathrm{~m}, 1 \mathrm{H}), 1.71$ $(\mathrm{s}, 2 \mathrm{H}), 1.44-1.37(\mathrm{~m}, 1 \mathrm{H}), 1.35-1.32(\mathrm{~m}, 2 \mathrm{H}), 1.29(\mathrm{~s}, 3 \mathrm{H}), 1.23-1.15(\mathrm{~m}, 1 \mathrm{H}), 0.87(\mathrm{t}, J=7.1 \mathrm{~Hz}$, $3 \mathrm{H})$.

${ }^{13}$ C NMR (101 MHz, CDCl $) \delta$ 178.14, 137.29, 133.41, 129.42, 128.51, 127.23, 126.24, 62.06, 52.17, 51.72, 31.86, 24.98, 20.86, 13.95.

HRMS (ESI) calcd. for $\mathrm{C}_{16} \mathrm{H}_{24} \mathrm{NO}_{2}{ }^{+}(\mathrm{M}+\mathrm{H})^{+}:$262.1807, Found: 262.1804

the ee value $\mathrm{was}>99 \%, \mathrm{t}_{\mathrm{r}}($ minor $)=13.028 \mathrm{~min}, \mathrm{t}_{\mathrm{r}}($ major $)=20.264 \mathrm{~min}($ Chiralcel $\mathrm{IC}, \lambda=220 \mathrm{~nm}$, hexanes $:{ }^{i} \mathrm{PrOH}=98: 2$, flow rate $=1.0 \mathrm{~mL} / \mathrm{min}$ ). 
$\mathrm{mV}$

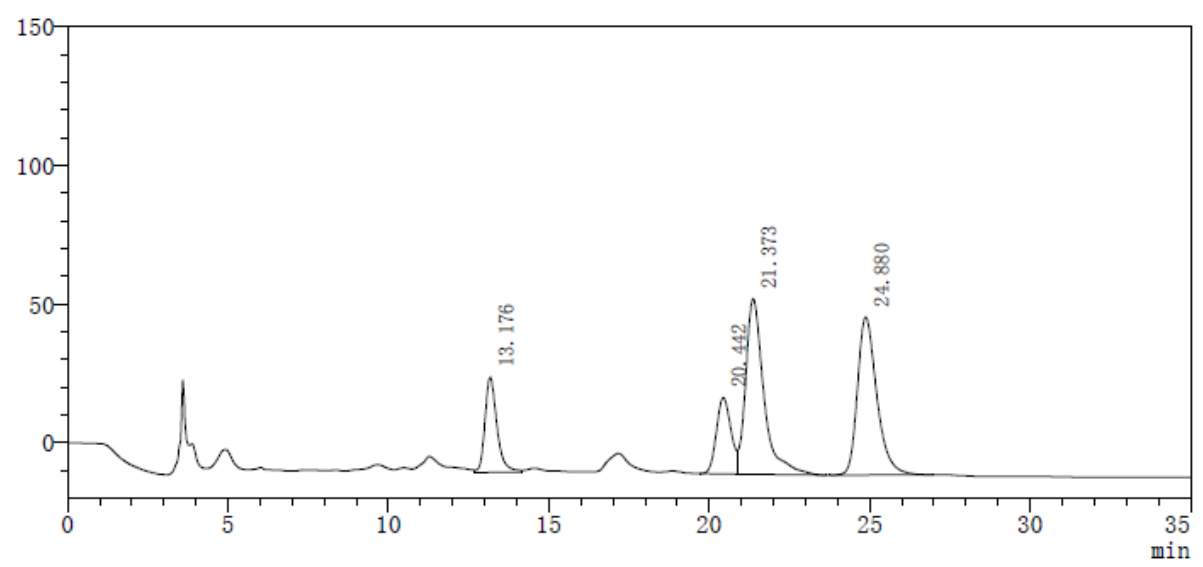

检测器A 220nm
\begin{tabular}{|c|c|c|c|c|c|}
\hline Peak\# & Time & Area & Height & Height\% & Area\% \\
\hline 1 & 13.176 & 891976 & 34189 & 18.793 & 13.379 \\
\hline 2 & 20.442 & 869062 & 27530 & 15.133 & 13.035 \\
\hline 3 & 21.373 & 2512550 & 63266 & 34.776 & 37.685 \\
\hline 4 & 24.880 & 2393606 & 56940 & 31.299 & 35.901 \\
\hline 总计 & & 6667194 & 181926 & 100.000 & 100.000 \\
\hline
\end{tabular}

$\mathrm{mV}$

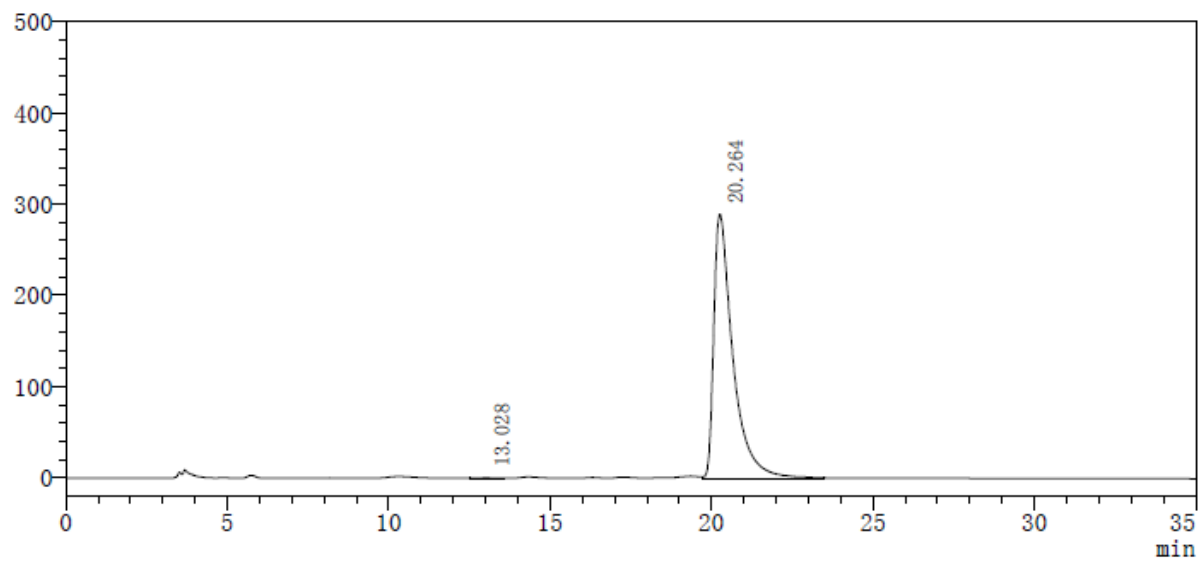

检测器A $220 \mathrm{~nm}$
\begin{tabular}{|c|c|c|c|c|c|}
\hline Peak\# & Time & Area & Height & Height $\%$ & Area\% \\
\hline 1 & 13.028 & 13948 & 569 & 0.196 & 0.117 \\
\hline 2 & 20.264 & 11927788 & 289226 & 99.804 & 99.883 \\
\hline 总计 & & 11941736 & 289795 & 100.000 & 100.000 \\
\hline
\end{tabular}

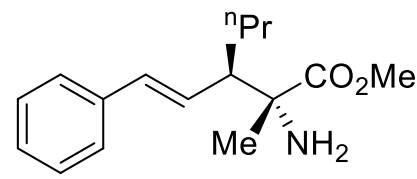

$(2 R, 3 R)-3 p a$

$(2 R, 3 R)$-methyl 2-amino-2-methyl-3-((E)-styryl)hexanoate 
Prepared according to General Procedure B using Pd-6 and $(R, R p)-\mathbf{L 2}-\mathbf{C u}$, Purification by flash chromatography (PE/EA = 5:1 to 1:1) afforded the product as a pale yellow oil, $24.5 \mathrm{mg}, 47 \%$ yield, $7: 1 \mathrm{dr},>99 \%$ ee, $\mathrm{R}_{\mathrm{f}}=0.35$ (PE/EA, 1:1).

$[\alpha]^{25} \mathrm{D}=11.3\left(\mathrm{c} 0.2, \mathrm{CHCl}_{3}\right)$

${ }^{1} \mathbf{H}$ NMR $\left(400 \mathrm{MHz}, \mathbf{C D C l}_{3}\right) \delta 7.35(\mathrm{~d}, J=7.7 \mathrm{~Hz}, 2 \mathrm{H}), 7.30(\mathrm{t}, J=7.5 \mathrm{~Hz}, 2 \mathrm{H}), 7.22(\mathrm{t}, J=7.3 \mathrm{~Hz}, 1 \mathrm{H})$, $6.40(\mathrm{~d}, J=15.7 \mathrm{~Hz}, 1 \mathrm{H}), 5.98(\mathrm{dd}, J=15.8,9.8 \mathrm{~Hz}, 1 \mathrm{H}), 3.70(\mathrm{~d}, J=1.7 \mathrm{~Hz}, 3 \mathrm{H}), 2.41(\mathrm{t}, J=10.5 \mathrm{~Hz}$, 1H), $1.70(\mathrm{~s}, 3 \mathrm{H}), 1.49-1.38(\mathrm{~m}, 2 \mathrm{H}), 1.33(\mathrm{~s}, 3 \mathrm{H}), 1.24-1.13(\mathrm{~m}, 2 \mathrm{H}), 0.90$ (t, $J=6.8 \mathrm{~Hz}, 3 \mathrm{H})$.

${ }^{13} \mathbf{C}$ NMR $\left(\mathbf{1 0 1} \mathbf{~ M H z}, \mathbf{C D C l}_{3}\right) \delta 177.15,137.22,133.25,129.63,128.53,127.32,126.24,61.19,52.35$, 52.08, 31.01, 23.62, 20.97, 13.99.

HRMS (ESI) calcd. for $\mathrm{C}_{16} \mathrm{H}_{24} \mathrm{NO}_{2}{ }^{+}(\mathrm{M}+\mathrm{H})^{+}:$262.1807, Found: 262.1804 the ee value $\mathrm{was}>99 \%, \mathrm{t}_{\mathrm{r}}($ minor $)=21.743 \mathrm{~min}, \mathrm{t}_{\mathrm{r}}$ (major) $=31.803 \mathrm{~min}$ (Chiralcel IC, $\lambda=220 \mathrm{~nm}$, hexanes $:{ }^{i} \mathrm{PrOH}=98: 2$, flow rate $=1.0 \mathrm{~mL} / \mathrm{min}$ ).

$\mathrm{mV}$

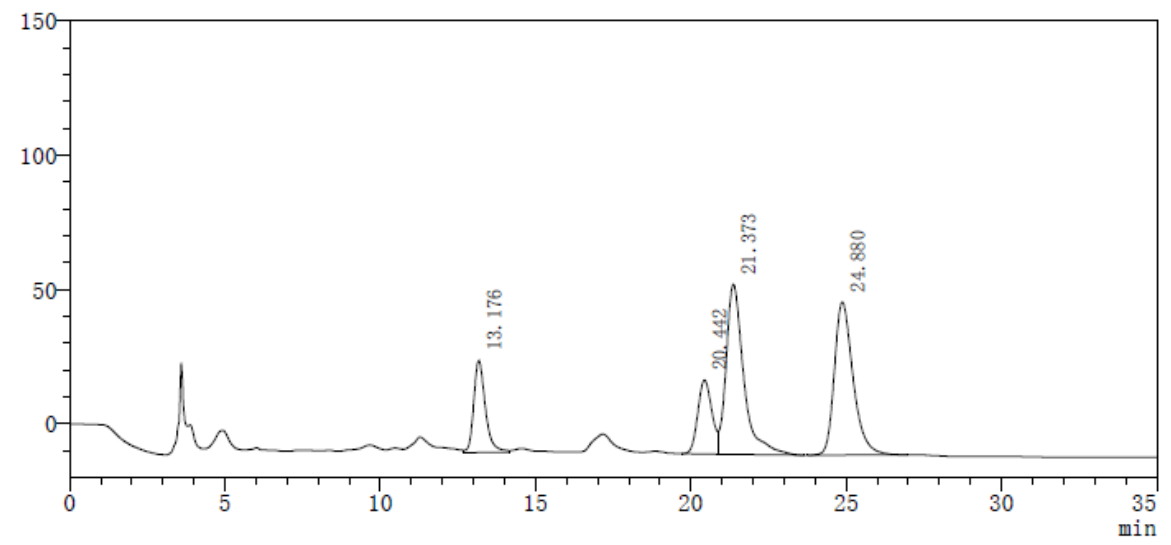

检测器A 220nm
\begin{tabular}{|c|c|c|c|c|c|}
\hline Peak\# & Time & Area & Height & Height\% & Area\% \\
\hline 1 & 13.176 & 891976 & 34189 & 18.793 & 13.379 \\
\hline 2 & 20.442 & 869062 & 27530 & 15.133 & 13.035 \\
\hline 3 & 21.373 & 2512550 & 63266 & 34.776 & 37.685 \\
\hline 4 & 24.880 & 2393606 & 56940 & 31.299 & 35.901 \\
\hline 总计 & & 6667194 & 181926 & 100.000 & 100.000 \\
\hline
\end{tabular}

$\mathrm{nV}$

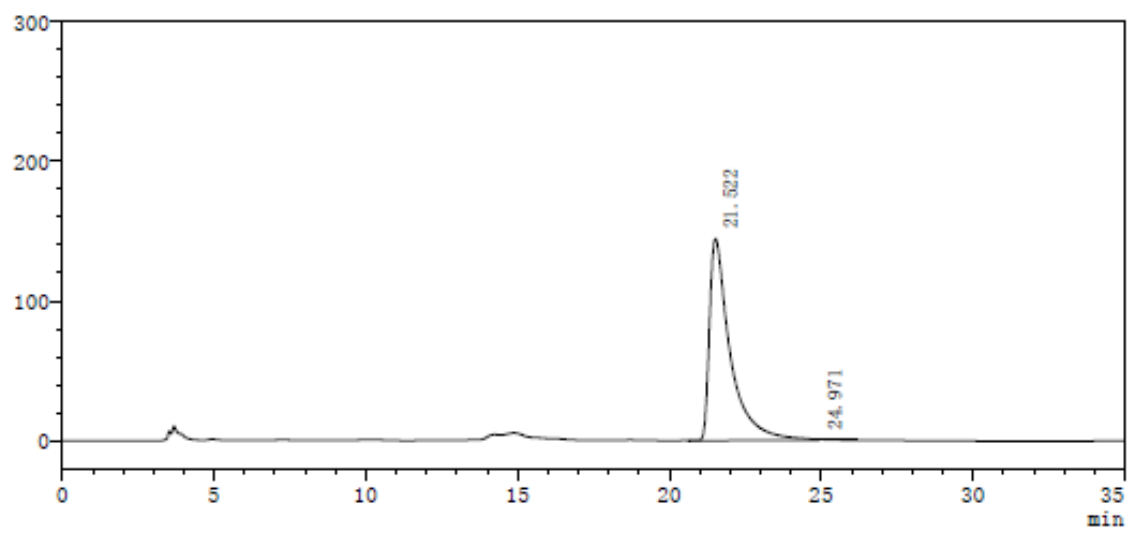

检测器A $220 \mathrm{~nm}$
\begin{tabular}{|c|c|c|c|c|c|}
\hline Peak\# & Time & Area & Height & Height\% & Area\% \\
\hline 1 & 21.522 & 6887574 & 144458 & 99.496 & 99.722 \\
\hline 2 & 24.971 & 19216 & 732 & 0.504 & 0.278 \\
\hline 总计 & & 6906790 & 145191 & 100.000 & 100.000 \\
\hline
\end{tabular}




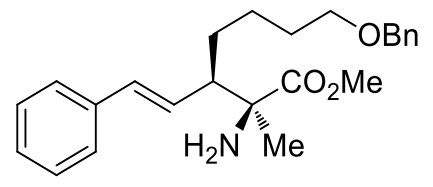

$(2 S, 3 R)-3 q a$

(2S,3R)-methyl 2-amino-7-(benzyloxy)-2-methyl-3-((E)-styryl)heptanoate

Prepared according to General Procedure B using Pd-6 and $(S, S p)$-L2-Cu, Purification by flash chromatography $(\mathrm{PE} / \mathrm{EA}=5: 1$ to $1: 1)$ afforded the product as a pale yellow oil, $34.3 \mathrm{mg}, 45 \%$ yield, $10: 1 \mathrm{dr}, 98 \%$ ee, $\mathrm{R}_{\mathrm{f}}=0.5$ (PE/EA, 1:2).

$[\alpha]^{25} \mathrm{D}=7.7\left(\mathrm{c} 0.2, \mathrm{CHCl}_{3}\right)$

${ }^{1}$ H NMR (400 MHz, CDCl $) \delta 7.38(\mathrm{~d}, J=7.4 \mathrm{~Hz}, 2 \mathrm{H}), 7.33-7.28(\mathrm{~m}, 5 \mathrm{H}), 7.27-7.19(\mathrm{~m}, 3 \mathrm{H})$, $6.43(\mathrm{~d}, J=15.8 \mathrm{~Hz}, 1 \mathrm{H}), 6.03(\mathrm{dd}, J=15.8,9.8 \mathrm{~Hz}, 1 \mathrm{H}), 4.46(\mathrm{~s}, 2 \mathrm{H}), 3.70(\mathrm{~s}, 3 \mathrm{H}), 3.42(\mathrm{td}, J=6.5$, $2.0 \mathrm{~Hz}, 2 \mathrm{H}), 2.47-2.37(\mathrm{~m}, 1 \mathrm{H}), 1.76(\mathrm{~s}, 2 \mathrm{H}), 1.72-1.60(\mathrm{~m}, 2 \mathrm{H}), 1.59-1.50(\mathrm{~m}, 1 \mathrm{H}), 1.47-1.33(\mathrm{~m}$, $3 \mathrm{H}), 1.29(\mathrm{~s}, 3 \mathrm{H})$.

${ }^{13}$ C NMR (101 MHz, CDCl 3 ) $\delta 177.96,138.59,137.23,133.67,129.14,128.53,128.35,127.64$, $127.49,127.29,126.29,72.89,70.24,60.96,52.22,51.93,29.59,29.48,24.94,24.43$.

HRMS (ESI) calcd. for $\mathrm{C}_{24} \mathrm{H}_{32} \mathrm{NO}_{3}{ }^{+}(\mathrm{M}+\mathrm{H})^{+}:$382.2382, Found: 382.2372

the ee value $\mathrm{w}$ as $98 \%, \mathrm{t}_{\mathrm{r}}($ minor $)=8.011 \mathrm{~min}, \mathrm{t}_{\mathrm{r}}($ major $)=8.838 \mathrm{~min}$ (Chiralcel $\mathrm{ID}, \lambda=220 \mathrm{~nm}$, hexanes : ${ }^{i} \mathrm{PrOH}=85: 15$, flow rate $\left.=1.0 \mathrm{~mL} / \mathrm{min}\right)$.

$\mathrm{mV}$

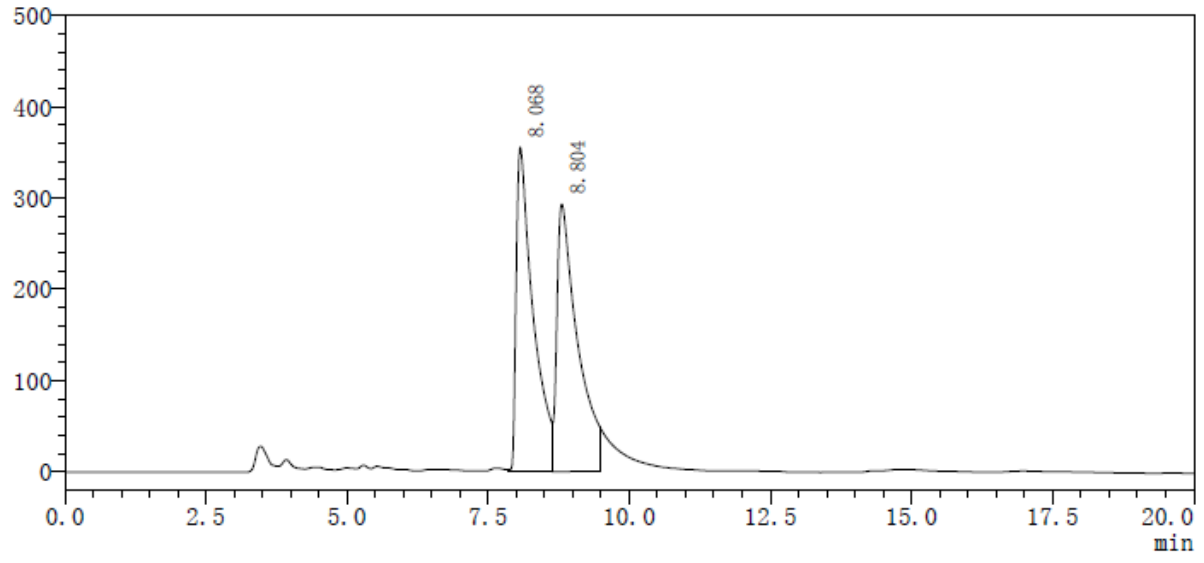

检测器A $220 \mathrm{~nm}$
\begin{tabular}{|c|c|c|c|c|c|}
\hline Peak\# & Time & Area & Height & Height\% & Area\% \\
\hline 1 & 8.068 & 7361419 & 354551 & 54.758 & 49.642 \\
\hline 2 & 8.804 & 7467470 & 292936 & 45.242 & 50.358 \\
\hline 总计 & & 14828889 & 647487 & 100.000 & 100.000 \\
\hline
\end{tabular}




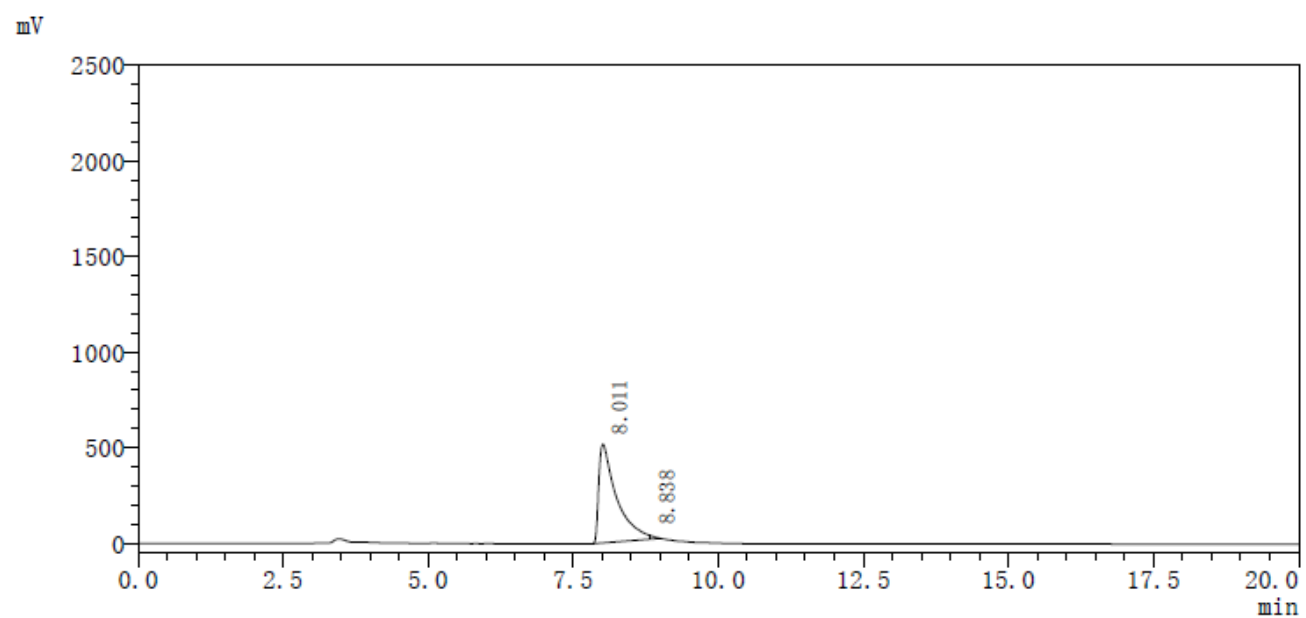

检测器A $220 \mathrm{~nm}$
\begin{tabular}{|c|c|c|c|c|c|}
\hline Peak\# & Time & Area & Height & Height\% & Area\% \\
\hline 1 & 8.011 & 10864731 & 514860 & 96.668 & 99.178 \\
\hline 2 & 8.838 & 90080 & 17748 & 3.332 & 0.822 \\
\hline 总计 & & 10954811 & 532608 & 100.000 & 100.000 \\
\hline
\end{tabular}<smiles>CC(=O)OCCCCC(/C=C/c1ccccc1)C(C)(N)C(=O)OCc1ccccc1</smiles>

(2R,3R)-methyl 2-amino-7-(benzyloxy)-2-methyl-3-((E)-styryl)heptanoate

Prepared according to General Procedure B using Pd-6 and $(R, R p)$-L2-Cu, Purification by flash chromatography (PE/EA = 5:1 to $1: 1)$ afforded the product as a pale yellow oil, $36 \mathrm{mg}, 47 \%$ yield, $5: 1 \mathrm{dr}, 97 \%$ ee, $\mathrm{R}_{\mathrm{f}}=0.4$ (PE/EA, 1:2).

$[\alpha]^{25}{ }_{\mathrm{D}}=-8.2\left(\mathrm{c} 0.2, \mathrm{CHCl}_{3}\right)$

${ }^{1}$ H NMR (400 MHz, $\left.\mathbf{C D C l}_{3}\right) \delta 7.35(\mathrm{~d}, J=7.1 \mathrm{~Hz}, 2 \mathrm{H}), 7.33-7.28(\mathrm{~m}, 5 \mathrm{H}), 7.27-7.20(\mathrm{~m}, 3 \mathrm{H})$, $6.40(\mathrm{~d}, J=15.8 \mathrm{~Hz}, 1 \mathrm{H}), 5.97(\mathrm{dd}, J=15.8,9.8 \mathrm{~Hz}, 1 \mathrm{H}), 4.46(\mathrm{~s}, 2 \mathrm{H}), 3.69(\mathrm{~s}, 3 \mathrm{H}), 3.43(\mathrm{t}, J=6.2$ $\mathrm{Hz}, 2 \mathrm{H}), 2.45-2.36(\mathrm{~m}, 1 \mathrm{H}), 1.68(\mathrm{~s}, 2 \mathrm{H}), 1.65-1.61(\mathrm{~m}, 1 \mathrm{H}), 1.61-1.51(\mathrm{~m}, 2 \mathrm{H}), 1.49-1.42(\mathrm{~m}$, $1 \mathrm{H}), 1.41-1.34(\mathrm{~m}, 2 \mathrm{H}), 1.32(\mathrm{~s}, 3 \mathrm{H})$.

${ }^{13}$ C NMR (101 MHz, CDCl 3 ) $\delta$ 177.54, 138.57, 137.14, 133.44, 129.40, 128.53, 128.33, 127.63, $127.48,127.36,126.27,72.86,70.23,61.19,52.51,52.12,29.65,28.60,24.53,23.64$.

HRMS (ESI) calcd. for $\mathrm{C}_{24} \mathrm{H}_{32} \mathrm{NO}_{3}{ }^{+}(\mathrm{M}+\mathrm{H})^{+}:$382.2382, Found: 382.2374

the ee value was $97 \%, \mathrm{t}_{\mathrm{r}}$ (minor) $=6.388 \mathrm{~min}, \mathrm{t}_{\mathrm{r}}$ (major) $=6.938 \min$ (Chiralcel IA, $\lambda=220 \mathrm{~nm}$, hexanes $:{ }^{i} \mathrm{PrOH}=85: 15$, flow rate $=1.0 \mathrm{~mL} / \mathrm{min}$ ). 
$\mathrm{mV}$

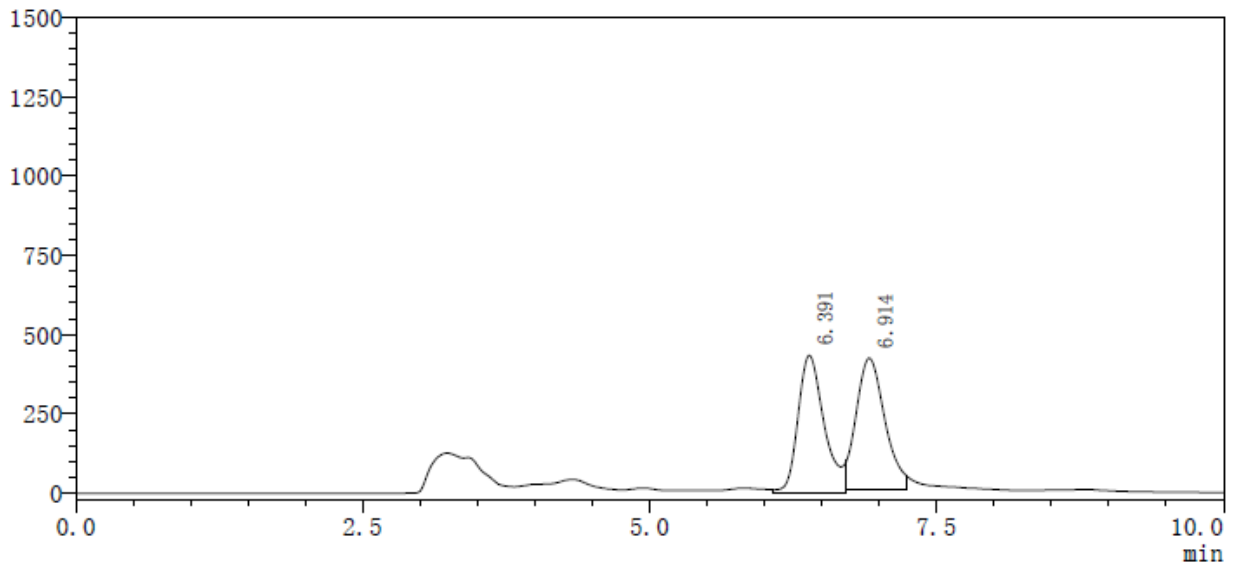

检测器 $\mathrm{A} 204 \mathrm{~nm}$

\begin{tabular}{|c|c|c|c|c|c|}
\hline Peak\# & Time & Area & Height & Height\% & Area\% \\
\hline 1 & 6.391 & 7122785 & 435591 & 51.168 & 49.763 \\
\hline 2 & 6.914 & 7190727 & 415712 & 48.832 & 50.237 \\
\hline 总计 & & 14313512 & 851303 & 100.000 & 100.000 \\
\hline
\end{tabular}

$\mathrm{mV}$

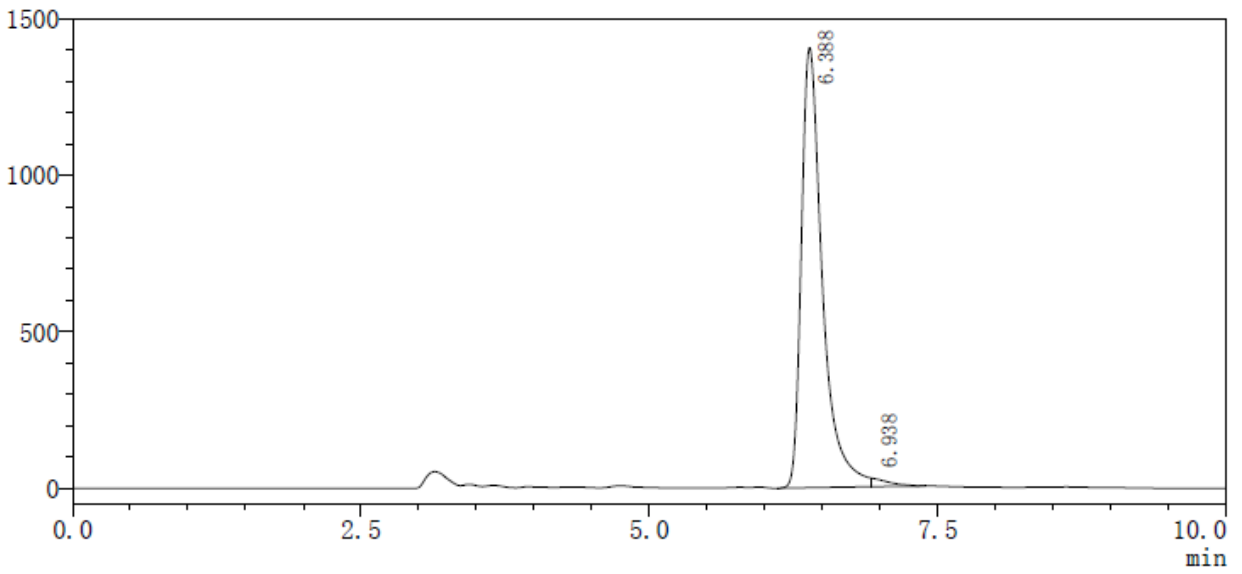

检测器A 204nm
\begin{tabular}{|c|c|c|c|c|c|}
\hline Peak\# & Time & Area & Height & Height\% & Area\% \\
\hline 1 & 6.388 & 17855696 & 1405778 & 98.137 & 98.497 \\
\hline 2 & 6.938 & 272412 & 26693 & 1.863 & 1.503 \\
\hline 总计 & & 18128108 & 1432471 & 100.000 & 100.000 \\
\hline
\end{tabular}<smiles>CC[C@@](N)(C(C)=O)[C@@H](C)/C=C/c1ccccc1</smiles>

$(2 S, 3 R)-3 \mathbf{a b}$

methyl (2S,3R,E)-2-amino-2-ethyl-3-methyl-5-phenylpent-4-enoate

Prepared according to General Procedure B using Pd-6 and $(S, S p)$-L2-Cu, Purification by flash chromatography (PE/EA $=5: 1$ to 1:1) afforded the product as a pale yellow solid, $42 \mathrm{mg}, 85 \%$ yield, m.p. $59-60{ }^{\circ} \mathrm{C},>20: 1 \mathrm{dr},>99 \%$ ee, $\mathrm{R}_{\mathrm{f}}=0.4(\mathrm{PE} / \mathrm{EA}, 1: 1)$.

$[\alpha]^{25}=70.6\left(\mathrm{c} 1.0, \mathrm{CHCl}_{3}\right)$ 
${ }^{1} \mathbf{H}$ NMR $\left(400 \mathrm{MHz}, \mathbf{C D C l}_{3}\right) \delta 7.39-7.34(\mathrm{~m}, 2 \mathrm{H}), 7.29(\mathrm{t}, J=7.5 \mathrm{~Hz}, 2 \mathrm{H}), 7.23-7.16(\mathrm{~m}, 1 \mathrm{H})$, $6.44(\mathrm{~d}, J=15.9 \mathrm{~Hz}, 1 \mathrm{H}), 6.18(\mathrm{dd}, J=15.9,9.2 \mathrm{~Hz}, 1 \mathrm{H}), 3.74(\mathrm{~s}, 3 \mathrm{H}), 2.70(\mathrm{dq}, J=9.2,6.9 \mathrm{~Hz}, 1 \mathrm{H})$, $1.81(\mathrm{dq}, J=14.7,7.5 \mathrm{~Hz}, 1 \mathrm{H}), 1.65-1.48(\mathrm{~m}, 3 \mathrm{H}), 1.03(\mathrm{~d}, J=6.8 \mathrm{~Hz}, 3 \mathrm{H}), 0.83(\mathrm{t}, J=7.5 \mathrm{~Hz}, 3 \mathrm{H})$. ${ }^{13}$ C NMR (101 MHz, CDCl $) \delta$ 177.19, 137.32, 131.65, 130.77, 128.51, 127.24, 126.22, 64.73, 52.08, 45.50, 31.80, 16.07, 8.45.

HRMS (ESI) calcd. for $\mathrm{C}_{15} \mathrm{H}_{22} \mathrm{NO}_{2}{ }^{+}(\mathrm{M}+\mathrm{H})^{+}:$248.1651, Found: 248.1648

the ee value was $>99 \%, \mathrm{t}_{\mathrm{r}}($ minor $)=9.775 \mathrm{~min}, \mathrm{t}_{\mathrm{r}}($ major $)=15.260 \mathrm{~min}($ Chiralcel $\mathrm{IC}, \lambda=220 \mathrm{~nm}$, hexanes $:{ }^{i} \mathrm{PrOH}=98: 2$, flow rate $\left.=1.0 \mathrm{~mL} / \mathrm{min}\right)$.

$\mathrm{mV}$

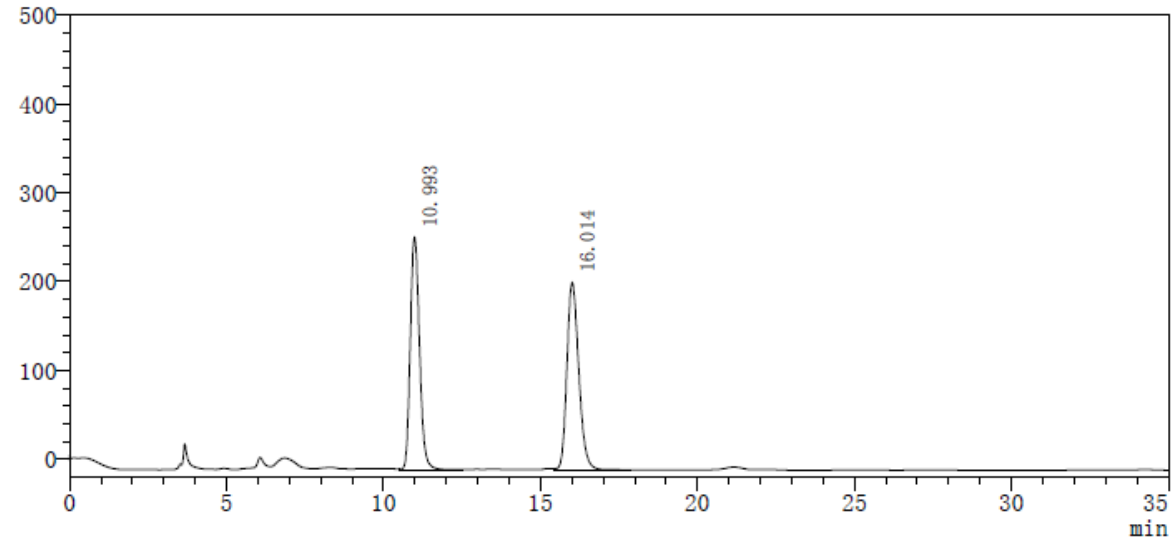

检测器A $220 \mathrm{~nm}$
\begin{tabular}{|c|c|c|c|c|c|}
\hline Peak\# & Time & Area & Height & Height\% & Area\% \\
\hline 1 & 10.993 & 5358905 & 261867 & 55.411 & 49.164 \\
\hline 2 & 16.014 & 5541078 & 210724 & 44.589 & 50.836 \\
\hline 总计 & & 10899982 & 472591 & 100.000 & 100.000 \\
\hline
\end{tabular}

$\mathrm{mV}$

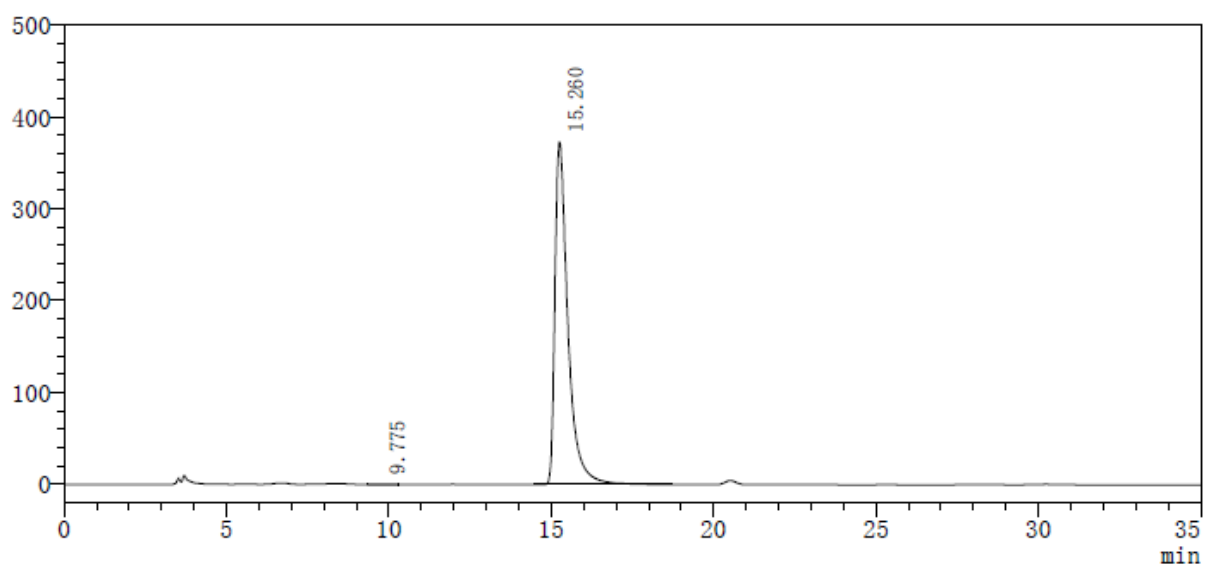

检测器A $220 \mathrm{~nm}$

\begin{tabular}{|c|c|c|c|c|c|}
\hline Peak\# & Time & Area & Height & Height\% & Area\% \\
\hline 1 & 9.775 & 7311 & 231 & 0.062 & 0.070 \\
\hline 2 & 15.260 & 10375216 & 372591 & 99.938 & 99.930 \\
\hline 总计 & & 10382527 & 372822 & 100.000 & 100.000 \\
\hline
\end{tabular}


<smiles>[R16][R16]#N</smiles>

methyl $(2 R, 3 R, E)$-2-amino-2-ethyl-3-methyl-5-phenylpent-4-enoate

Prepared according to General Procedure B using Pd-6 and $(R, R p)-\mathbf{L 2}-\mathbf{C u}$, Purification by flash chromatography $(\mathrm{PE} / \mathrm{EA}=5: 1$ to $1: 1)$ afforded the product as a pale yellow oil, $37 \mathrm{mg}, 76 \%$ yield, $13: 1 \mathrm{dr},>99 \%$ ee, $\mathrm{R}_{\mathrm{f}}=0.35$ (PE/EA, 1:1).

$[\alpha]^{25}{ }_{\mathrm{D}}=30.4\left(\mathrm{c} 0.5, \mathrm{CHCl}_{3}\right)$

${ }^{1} \mathbf{H}$ NMR (400 MHz, $\left.\mathbf{C D C l}_{3}\right) \delta 7.37-7.28(\mathrm{~m}, 4 \mathrm{H}), 7.25-7.15(\mathrm{~m}, 1 \mathrm{H}), 6.39(\mathrm{dd}, J=15.7,2.8 \mathrm{~Hz}$, $1 \mathrm{H}), 6.13(\mathrm{ddd}, J=15.9,8.5,2.9 \mathrm{~Hz}, 1 \mathrm{H}), 3.71(\mathrm{~s}, 3 \mathrm{H}), 2.75-2.66(\mathrm{~m}, 1 \mathrm{H}), 1.92-1.78(\mathrm{~m}, 1 \mathrm{H}), 1.75$ $-1.58(\mathrm{~m}, 3 \mathrm{H}), 1.13(\mathrm{dd}, J=6.9,2.9 \mathrm{~Hz}, 3 \mathrm{H}), 0.87(\mathrm{td}, J=7.5,2.9 \mathrm{~Hz}, 3 \mathrm{H})$.

${ }^{13}$ C NMR (101 MHz, $\left.\mathbf{C D C l}_{3}\right) \delta$ 176.89, 137.36, 131.30, 131.10, 128.50, 127.26, 126.23, 65.04, 51.98, $45.21,30.68,14.02,8.54$.

HRMS (ESI) calcd. for $\mathrm{C}_{15} \mathrm{H}_{22} \mathrm{NO}_{2}{ }^{+}(\mathrm{M}+\mathrm{H})^{+}:$:248.1651, Found: 248.1646

the ee value $\mathrm{was}>99 \%, \mathrm{t}_{\mathrm{r}}$ (major) $=15.709 \mathrm{~min}, \mathrm{t}_{\mathrm{r}}($ minor $)=26.540 \mathrm{~min}$ (Chiralcel $\mathrm{IC}, \lambda=220 \mathrm{~nm}$, hexanes $:{ }^{i} \mathrm{PrOH}=98: 2$, flow rate $=1.0 \mathrm{~mL} / \mathrm{min}$ ).

$\mathrm{mV}$

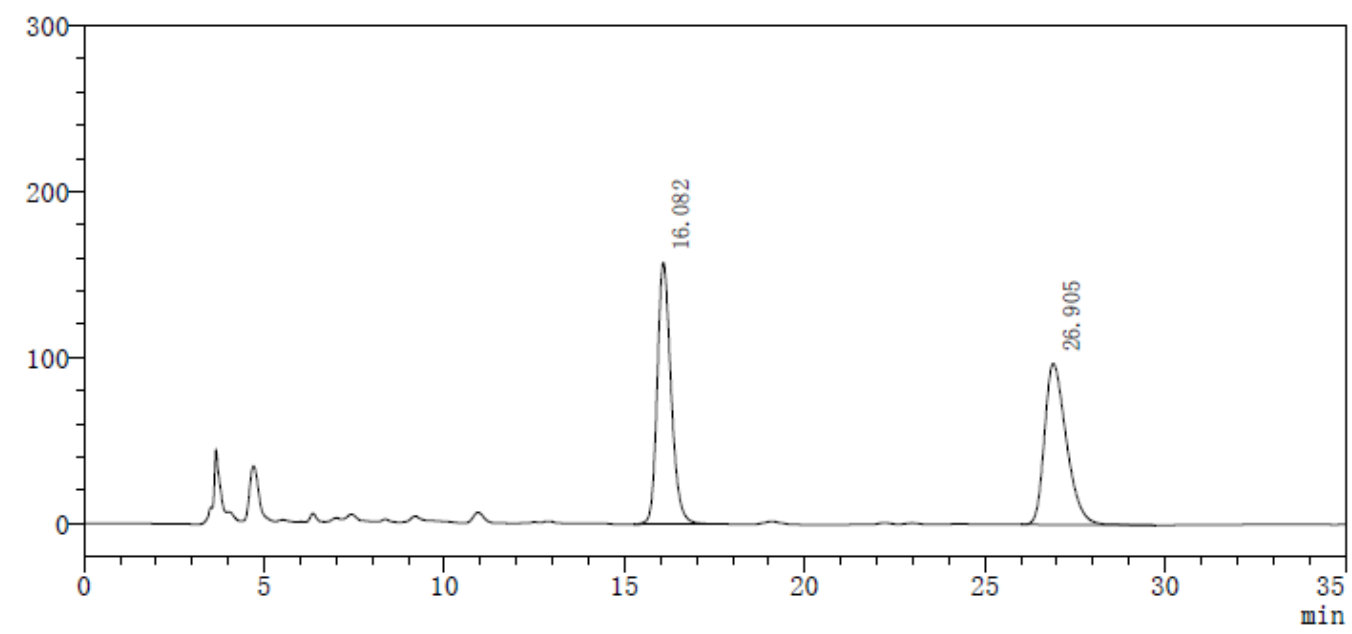

检测器A $220 \mathrm{~nm}$

\begin{tabular}{|c|c|c|c|c|c|}
\hline Peak\# & Time & Area & Height & Height\% & Area\% \\
\hline 1 & 16.082 & 4197758 & 157665 & 61.895 & 50.589 \\
\hline 2 & 26.905 & 4099991 & 97063 & 38.105 & 49.411 \\
\hline 总计 & & 8297749 & 254728 & 100.000 & 100.000 \\
\hline
\end{tabular}


$\mathrm{mV}$

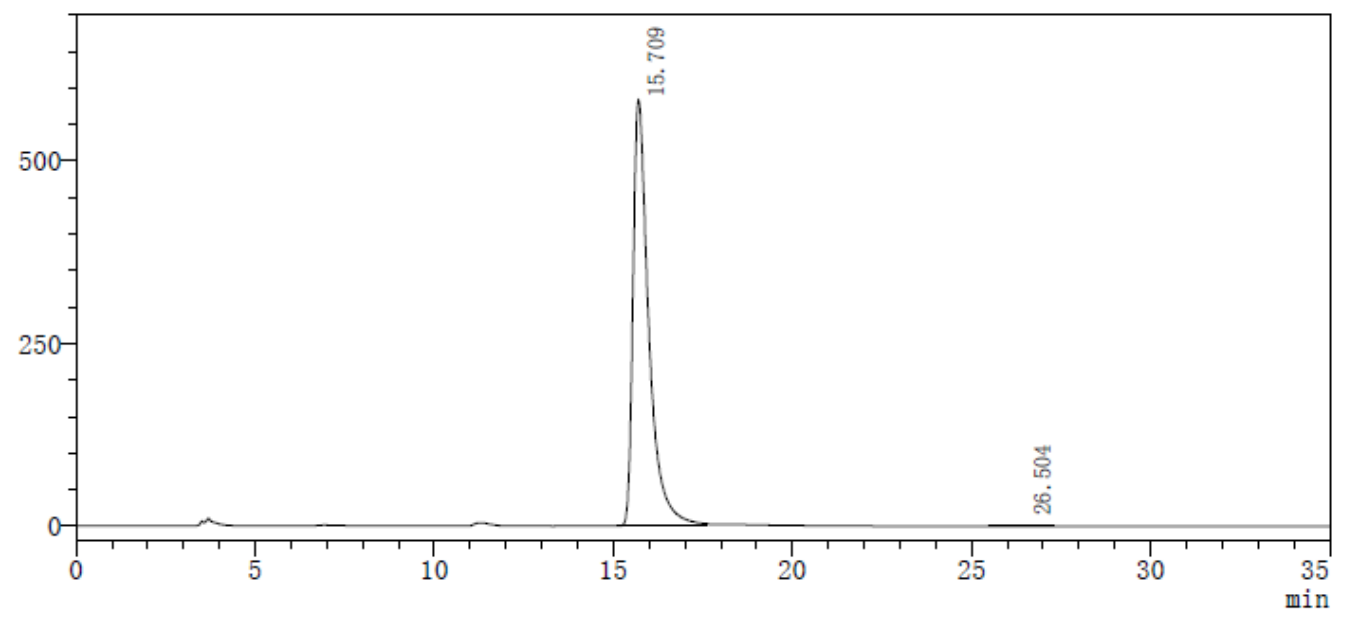

检测器A $220 \mathrm{~nm}$
\begin{tabular}{|c|c|c|c|c|c|}
\hline Peak\# & Time & Area & Height & Height\% & Area\% \\
\hline 1 & 15.709 & 17965259 & 583466 & 99.977 & 99.944 \\
\hline 2 & 26.504 & 10102 & 134 & 0.023 & 0.056 \\
\hline 总计 & & 17975361 & 583600 & 100.000 & 100.000 \\
\hline
\end{tabular}<smiles>CC(=O)C(N)(C(=O)P)C(C)/C=C/c1ccccc1</smiles>

$(2 S, 3 R)-3 a c$

\section{methyl $(2 S, 3 R, E)$-2-amino-3-methyl-5-phenyl-2-propylpent-4-enoate}

Prepared according to General Procedure B using Pd-6 and $(S, S p)$-L2-Cu, Purification by flash chromatography (PE/EA $=5: 1$ to 1:1) afforded the product as a pale yellow solid, $46 \mathrm{mg}, 86 \%$ yield, m.p. $68-69^{\circ} \mathrm{C},>20: 1 \mathrm{dr},>99 \%$ ee, $\mathrm{R}_{\mathrm{f}}=0.35$ (PE/EA, 2:1).

$[\alpha]^{25} \mathrm{D}=78.6\left(\mathrm{c} 1.0, \mathrm{CHCl}_{3}\right)$

${ }^{1}$ H NMR (400 MHz, CDCl $) \delta 7.38(\mathrm{~d}, J=8.3 \mathrm{~Hz}, 2 \mathrm{H}), 7.30(\mathrm{t}, J=7.5 \mathrm{~Hz}, 2 \mathrm{H}), 7.23-7.17(\mathrm{~m}, 1 \mathrm{H})$, $6.44(\mathrm{~d}, J=15.8 \mathrm{~Hz}, 1 \mathrm{H}), 6.18(\mathrm{dd}, J=15.8,9.1 \mathrm{~Hz}, 1 \mathrm{H}), 3.73(\mathrm{~s}, 3 \mathrm{H}), 2.68(\mathrm{dt}, J=13.7,6.9 \mathrm{~Hz}, 1 \mathrm{H})$, $1.73(\mathrm{td}, J=12.9,4.5 \mathrm{~Hz}, 1 \mathrm{H}), 1.58-1.45(\mathrm{~m}, 3 \mathrm{H}), 1.43-1.31(\mathrm{~m}, 1 \mathrm{H}), 1.14-0.99(\mathrm{~m}, 4 \mathrm{H}), 0.88(\mathrm{t}$, $J=7.2 \mathrm{~Hz}, 3 \mathrm{H})$

${ }^{13}$ C NMR (101 MHz, $\left.\mathbf{C D C l}_{3}\right) \delta 177.36,137.35,131.64,130.77,128.52,127.23,126.24,64.29,52.08$, $45.74,41.26,17.53,16.00,14.39$.

HRMS (ESI) calcd. for $\mathrm{C}_{16} \mathrm{H}_{24} \mathrm{NO}_{2}{ }^{+}(\mathrm{M}+\mathrm{H})^{+}:$:262.1807, Found: 262.1807

the ee value $\mathrm{was}>99 \%, \mathrm{t}_{\mathrm{r}}($ minor $)=10.217 \mathrm{~min}, \mathrm{t}_{\mathrm{r}}($ major $)=13.784 \mathrm{~min}($ Chiralcel $\mathrm{IC}, \lambda=220 \mathrm{~nm}$, hexanes $:{ }^{i} \mathrm{PrOH}=98: 2$, flow rate $=1.0 \mathrm{~mL} / \mathrm{min}$ ). 
$\mathrm{mV}$

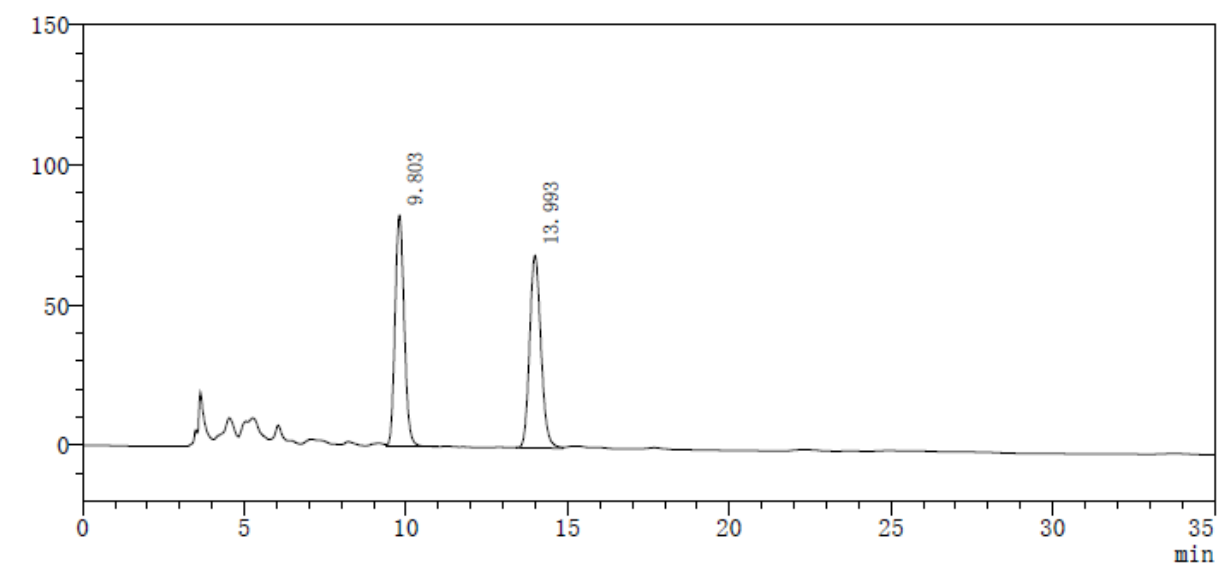

检测器A $220 \mathrm{~nm}$
\begin{tabular}{|c|c|c|c|c|c|}
\hline Peak\# & Time & Area & Height & Height\% & Area\% \\
\hline 1 & 9.803 & 1641269 & 82588 & 54.621 & 49.565 \\
\hline 2 & 13.993 & 1670059 & 68613 & 45.379 & 50.435 \\
\hline 总计 & & 3311328 & 151202 & 100.000 & 100.000 \\
\hline
\end{tabular}

$\mathrm{mV}$

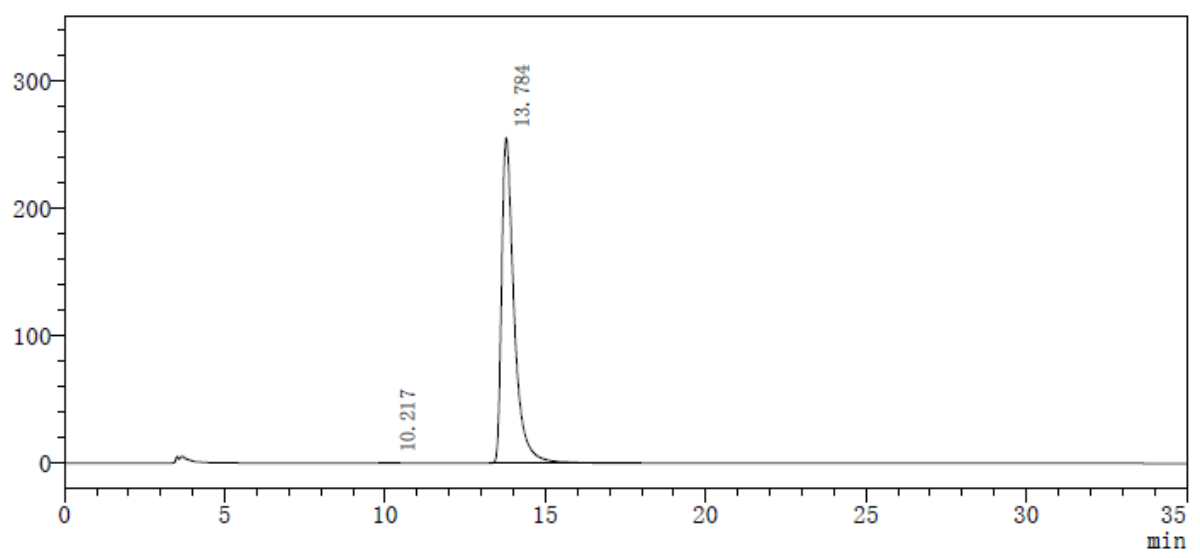

检测器A $220 \mathrm{~nm}$
\begin{tabular}{|c|c|c|c|c|c|}
\hline Peak\# & Time & Area & Height & Height $\%$ & Area\% \\
\hline 1 & 10.217 & 1295 & 60 & 0.024 & 0.019 \\
\hline 2 & 13.784 & 6954143 & 255035 & 99.976 & 99.981 \\
\hline 总计 & & 6955437 & 255096 & 100.000 & 100.000 \\
\hline
\end{tabular}<smiles>COC(=O)C(N)(OC(C)=O)C(C)/C=C/c1ccccc1</smiles>

$(2 S, 3 R)-3 \mathbf{a d}$

methyl ( $S)$-2-amino-2-((R,E)-4-phenylbut-3-en-2-yl)hexanoate

Prepared according to General Procedure B using Pd-6 and $(R, R p)$-L2-Cu, Purification by flash chromatography $(\mathrm{PE} / \mathrm{EA}=10: 1$ to 3:1) afforded the product as a pale yellow oil, $48.4 \mathrm{mg}, 88 \%$ yield, $>20: 1 \mathrm{dr},>99 \%$ ee, $\mathrm{R}_{\mathrm{f}}=0.4$ (PE/EA, 3:1). 
$[\alpha]^{25} \mathrm{D}=77.9\left(\mathrm{c} 1.0, \mathrm{CHCl}_{3}\right)$

${ }^{1}$ H NMR (400 MHz, CDCl $) \delta 7.41-7.35(\mathrm{~m}, 2 \mathrm{H}), 7.30(\mathrm{t}, J=7.6 \mathrm{~Hz}, 2 \mathrm{H}), 7.25-7.17(\mathrm{~m}, 1 \mathrm{H})$, $6.44(\mathrm{~d}, J=15.9 \mathrm{~Hz}, 1 \mathrm{H}), 6.18(\mathrm{dd}, J=15.8,9.2 \mathrm{~Hz}, 1 \mathrm{H}), 3.74(\mathrm{~s}, 3 \mathrm{H}), 2.69(\mathrm{dq}, J=9.2,6.9 \mathrm{~Hz}, 1 \mathrm{H})$, $1.76(\mathrm{ddd}, J=13.6,11.8,4.5 \mathrm{~Hz}, 1 \mathrm{H}), 1.60(\mathrm{~s}, 2 \mathrm{H}), 1.55-1.45(\mathrm{~m}, 1 \mathrm{H}), 1.29(\mathrm{dq}, J=18.8,7.5 \mathrm{~Hz}$, $3 \mathrm{H}), 1.04-1.09(\mathrm{~m}, 4 \mathrm{H}), 0.87(\mathrm{t}, J=7.2 \mathrm{~Hz}, 3 \mathrm{H})$.

${ }^{13} \mathbf{C}$ NMR (101 MHz, $\left.\mathbf{C D C l}_{3}\right) \delta 177.37,137.35,131.66,130.75,128.52,127.24,126.24,64.26,52.10$, 45.72, 38.75, 26.44, 23.00, 16.03, 14.04 .

HRMS (ESI) calcd. for $\mathrm{C}_{17} \mathrm{H}_{26} \mathrm{NO}_{2}{ }^{+}(\mathrm{M}+\mathrm{H})^{+}:$276.1964, Found: 276.1961

the ee value was $>99 \%, \mathrm{t}_{\mathrm{r}}($ minor $)=9.878 \mathrm{~min}, \mathrm{t}_{\mathrm{r}}($ major $)=12.238 \mathrm{~min}$ (Chiralcel $\mathrm{IC}, \lambda=220 \mathrm{~nm}$, hexanes $:{ }^{i} \mathrm{PrOH}=98: 2$, flow rate $\left.=1.0 \mathrm{~mL} / \mathrm{min}\right)$.

$\mathrm{mV}$

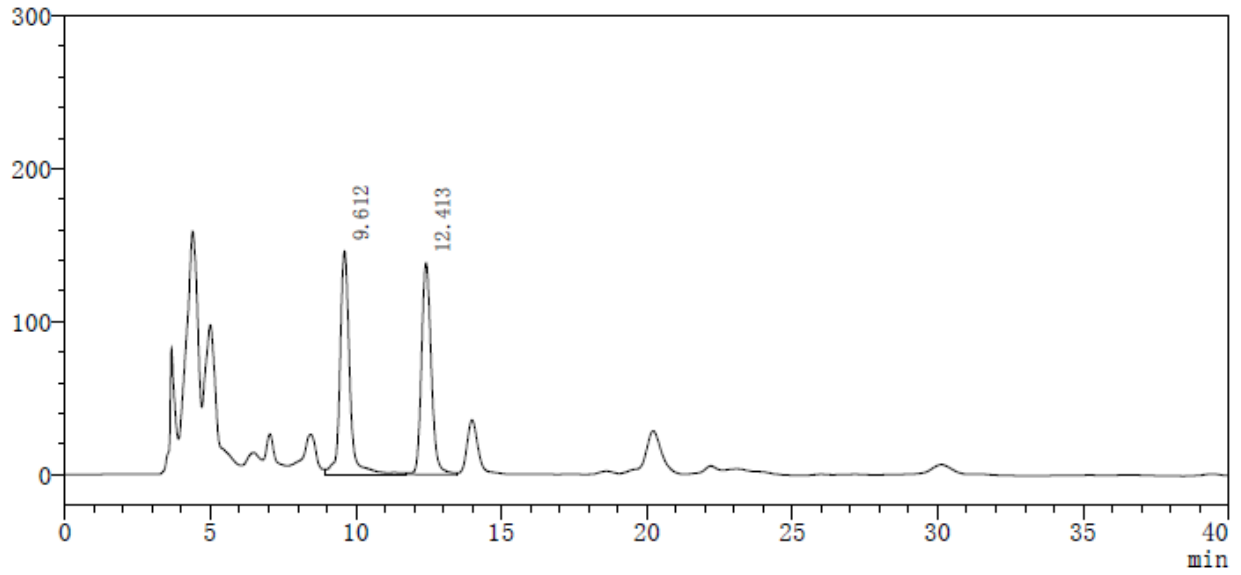

检测器A $220 \mathrm{~nm}$
\begin{tabular}{|c|c|c|c|c|c|}
\hline Peak\# & Time & Area & Height & Height $\%$ & Area\% \\
\hline 1 & 9.612 & 3387854 & 146157 & 51.358 & 51.103 \\
\hline 2 & 12.413 & 3241634 & 138426 & 48.642 & 48.897 \\
\hline 总计 & & 6629488 & 284583 & 100.000 & 100.000 \\
\hline
\end{tabular}

$\mathrm{mV}$

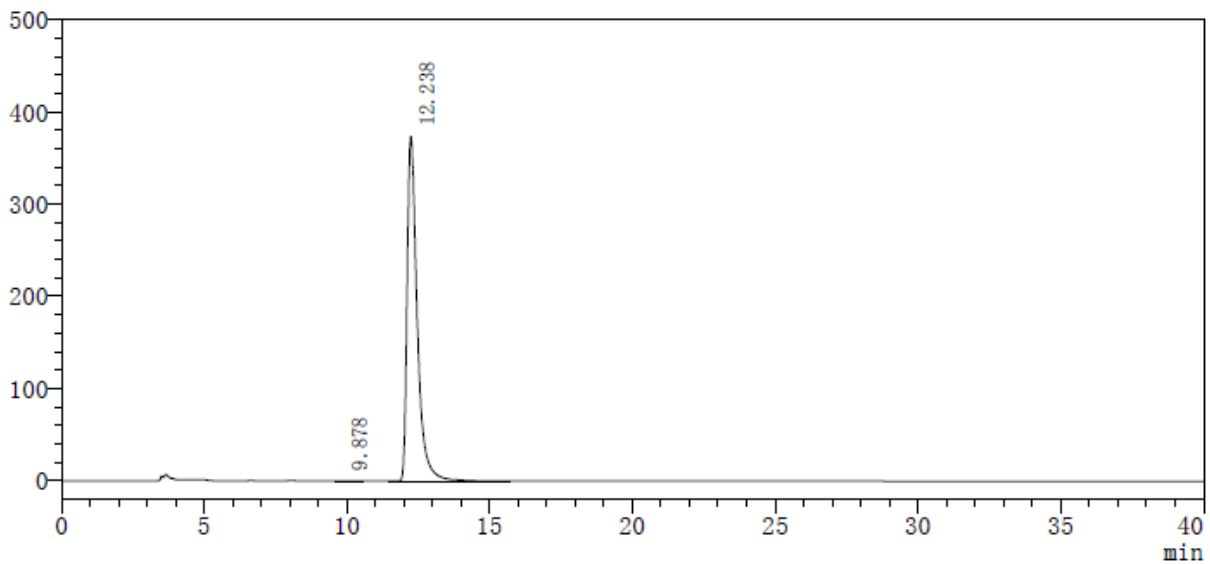

\begin{tabular}{l} 
检测器A $220 \mathrm{~nm}$ \\
\hline Peak\#
\end{tabular}


<smiles>COC(=O)[C@](N)(CCc1ccccc1)C(C)/C=C/c1ccccc1</smiles>

$(2 S, 3 R)-3 \mathbf{a e}$

methyl (2S,3R,E)-2-amino-3-methyl-2-phenethyl-5-phenylpent-4-enoate

Prepared according to General Procedure B using Pd-6 and $(S, S p)$-L2-Cu, Purification by flash chromatography (PE/EA $=5: 1$ to 1:1) afforded the product as a pale yellow solid, $60 \mathrm{mg}, 95 \%$ yield, m.p. $55-57^{\circ} \mathrm{C},>20: 1 \mathrm{dr},>99 \%$ ee, $\mathrm{R}_{\mathrm{f}}=0.4$ (PE/EA, 1:1).

$[\alpha]^{25}=98.4\left(\mathrm{c} 1.0, \mathrm{CHCl}_{3}\right)$

${ }^{1}$ H NMR (400 MHz, $\left.\mathbf{C D C l}_{3}\right) \delta 7.37-7.33(\mathrm{~m}, 2 \mathrm{H}), 7.28(\mathrm{t}, J=7.1 \mathrm{~Hz}, 2 \mathrm{H}), 7.27-7.22(\mathrm{~m}, 2 \mathrm{H})$,

$7.22-7.19(\mathrm{~m}, 1 \mathrm{H}), 7.19-7.15(\mathrm{~m}, 1 \mathrm{H}), 7.16-7.13(\mathrm{~m}, 2 \mathrm{H}), 6.44(\mathrm{~d}, J=15.8 \mathrm{~Hz}, 1 \mathrm{H}), 6.17(\mathrm{dd}, J=$ 15.9, $9.1 \mathrm{~Hz}, 1 \mathrm{H}), 3.75(\mathrm{~s}, 3 \mathrm{H}), 2.79-2.64(\mathrm{~m}, 2 \mathrm{H}), 2.40(\mathrm{td}, J=12.7,4.7 \mathrm{~Hz}, 1 \mathrm{H}), 2.15-2.06(\mathrm{~m}$, $1 \mathrm{H}), 1.85$ (ddd, $J=13.6,12.0,5.2 \mathrm{~Hz}, 1 \mathrm{H}), 1.63(\mathrm{~s}, 2 \mathrm{H}), 1.05(\mathrm{~d}, J=6.9 \mathrm{~Hz}, 3 \mathrm{H})$.

${ }^{13}$ C NMR (101 MHz, CDCl $) \delta 176.95,141.63,137.21,131.97,130.32,128.56,128.46,127.35$, $126.30,126.01,64.30,52.24,45.85,40.93,30.84,15.98$.

HRMS (ESI) calcd. for $\mathrm{C}_{21} \mathrm{H}_{26} \mathrm{NO}_{2}{ }^{+}(\mathrm{M}+\mathrm{H})^{+}:$324.1964, Found: 324.1956

the ee value $\mathrm{was}>99 \%, \mathrm{t}_{\mathrm{r}}($ minor $)=17.946 \mathrm{~min}, \mathrm{t}_{\mathrm{r}}($ major $)=41.947 \mathrm{~min}$ (Chiralcel $\mathrm{IC}, \lambda=220 \mathrm{~nm}$, hexanes $:{ }^{i} \mathrm{PrOH}=98: 2$, flow rate $=0.8 \mathrm{~mL} / \mathrm{min}$ ).

$\mathrm{mV}$

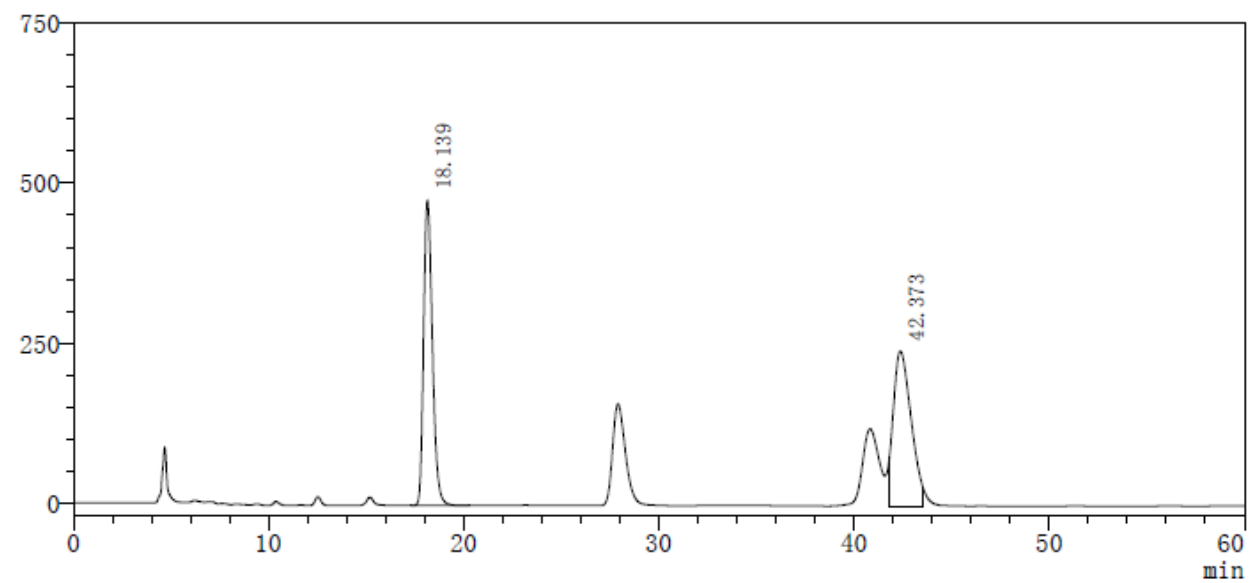

检测器A $220 \mathrm{~nm}$
\begin{tabular}{|c|c|c|c|c|c|}
\hline Peak\# & Time & Area & Height & Height\% & Area\% \\
\hline 1 & 18.139 & 14771683 & 476433 & 66.290 & 49.348 \\
\hline 2 & 42.373 & 15162197 & 242277 & 33.710 & 50.652 \\
\hline 总计 & & 29933880 & 718711 & 100.000 & 100.000 \\
\hline
\end{tabular}


$\mathrm{mV}$

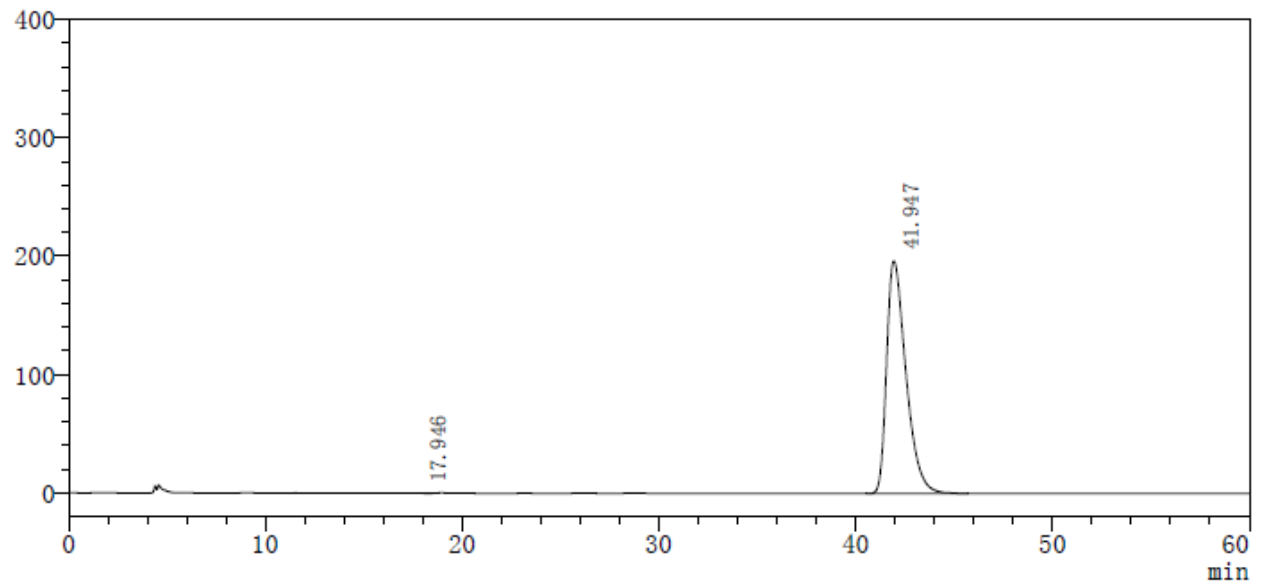

检测器A $220 \mathrm{~nm}$
\begin{tabular}{|c|c|c|c|c|c|}
\hline Peak\# & Time & Area & Height & Height\% & Area\% \\
\hline 1 & 17.946 & 1522 & 78 & 0.040 & 0.011 \\
\hline 2 & 41.947 & 13385564 & 196633 & 99.960 & 99.989 \\
\hline 总计 & & 13387085 & 196711 & 100.000 & 100.000 \\
\hline
\end{tabular}<smiles>CC(=O)C(N)(Br)C(C)/C=C/c1ccccc1</smiles>

$(2 S, 3 R)-3$ af

methyl $(2 S, 3 R, E)$-2-amino-2-benzyl-3-methyl-5-phenylpent-4-enoate

Prepared according to General Procedure B using Pd-6 and $(S, S p)$-L2-Cu, Purification by flash chromatography (PE/EA $=5: 1$ to $1: 1)$ afforded the product as a pale yellow solid, $28.5 \mathrm{mg}, 46 \%$ yield, m.p. $72-73{ }^{\circ} \mathrm{C},>20: 1 \mathrm{dr}, 98 \%$ ee, $\mathrm{R}_{\mathrm{f}}=0.4(\mathrm{PE} / \mathrm{EA}, 1: 1)$.

$[\alpha]^{25} \mathrm{D}=62.9\left(\mathrm{c} 0.5, \mathrm{CHCl}_{3}\right)$

${ }^{1} \mathbf{H}$ NMR $\left(400 \mathrm{MHz}, \mathbf{C D C l}_{3}\right) \delta 7.45-7.39(\mathrm{~m}, 2 \mathrm{H}), 7.32(\mathrm{t}, J=7.5 \mathrm{~Hz}, 2 \mathrm{H}), 7.28-7.20(\mathrm{~m}, 4 \mathrm{H})$,

$7.13-7.07(\mathrm{~m}, 2 \mathrm{H}), 6.53(\mathrm{~d}, J=15.9 \mathrm{~Hz}, 1 \mathrm{H}), 6.28(\mathrm{dd}, J=15.9,9.3 \mathrm{~Hz}, 1 \mathrm{H}), 3.71(\mathrm{~s}, 3 \mathrm{H}), 3.19(\mathrm{~d}, J$

$=13.3 \mathrm{~Hz}, 1 \mathrm{H}), 2.85(\mathrm{dq}, J=9.4,6.9 \mathrm{~Hz}, 1 \mathrm{H}), 2.78(\mathrm{~d}, J=13.3 \mathrm{~Hz}, 1 \mathrm{H}), 1.55(\mathrm{~s}, 2 \mathrm{H}), 1.08(\mathrm{~d}, J=6.9$ $\mathrm{Hz}, 3 \mathrm{H})$.

${ }^{13}$ C NMR (101 MHz, CDCl $) \delta 176.72,137.28,136.66,132.15,130.68,129.82,128.57,128.45$, 127.36, 126.95, 126.33, 65.36, 51.97, 46.04, 44.68, 16.39 .

HRMS (ESI) calcd. for $\mathrm{C}_{20} \mathrm{H}_{24} \mathrm{NO}_{2}{ }^{+}(\mathrm{M}+\mathrm{H})^{+}: 310.1807$, Found: 310.1803

the ee value $\mathrm{was} 98 \%, \mathrm{t}_{\mathrm{r}}($ major $)=11.042 \mathrm{~min}, \mathrm{t}_{\mathrm{r}}($ minor $)=17.939 \min ($ Chiralcel $\mathrm{IC}, \lambda=220 \mathrm{~nm}$, hexanes $:{ }^{i} \mathrm{PrOH}=98: 2$, flow rate $\left.=1.0 \mathrm{~mL} / \mathrm{min}\right)$. 
$\mathrm{mV}$

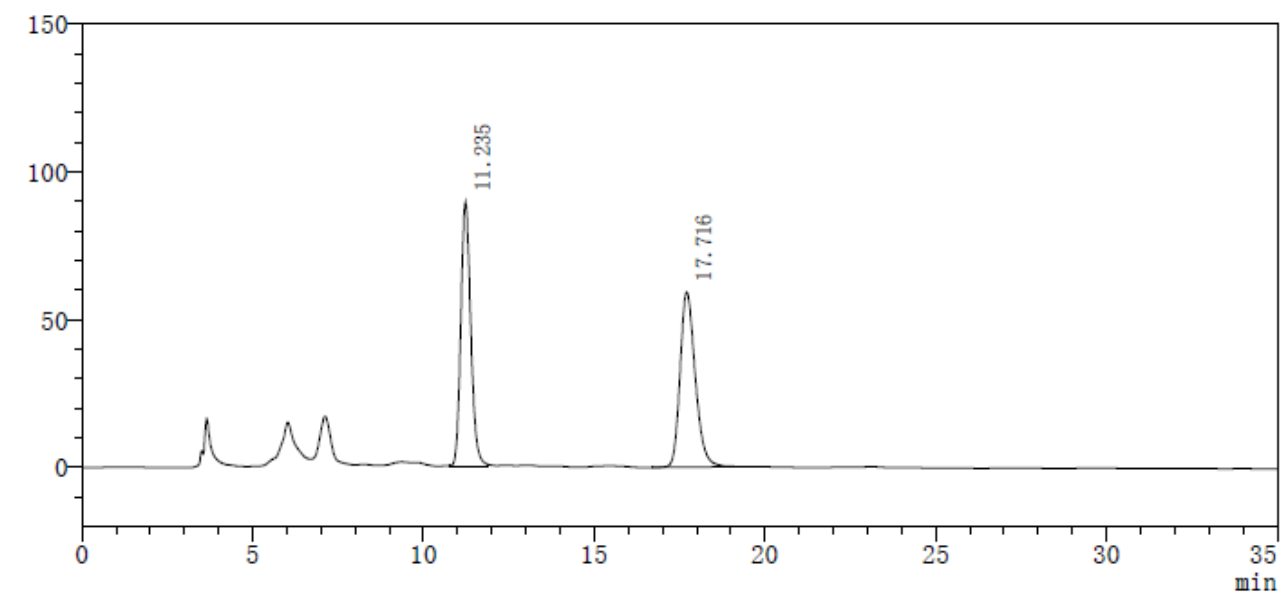

\begin{tabular}{|c|c|c|c|c|c|}
\hline Peak\# & Time & Area & Height & Height\% & Area $\%$ \\
\hline 1 & 11.235 & 1836707 & 89784 & 60.258 & 50.085 \\
\hline 2 & 17.716 & 1830498 & 59215 & 39.742 & 49.915 \\
\hline 总计 & & 3667205 & 149000 & 100.000 & 100.000 \\
\hline
\end{tabular}

$\mathrm{mV}$

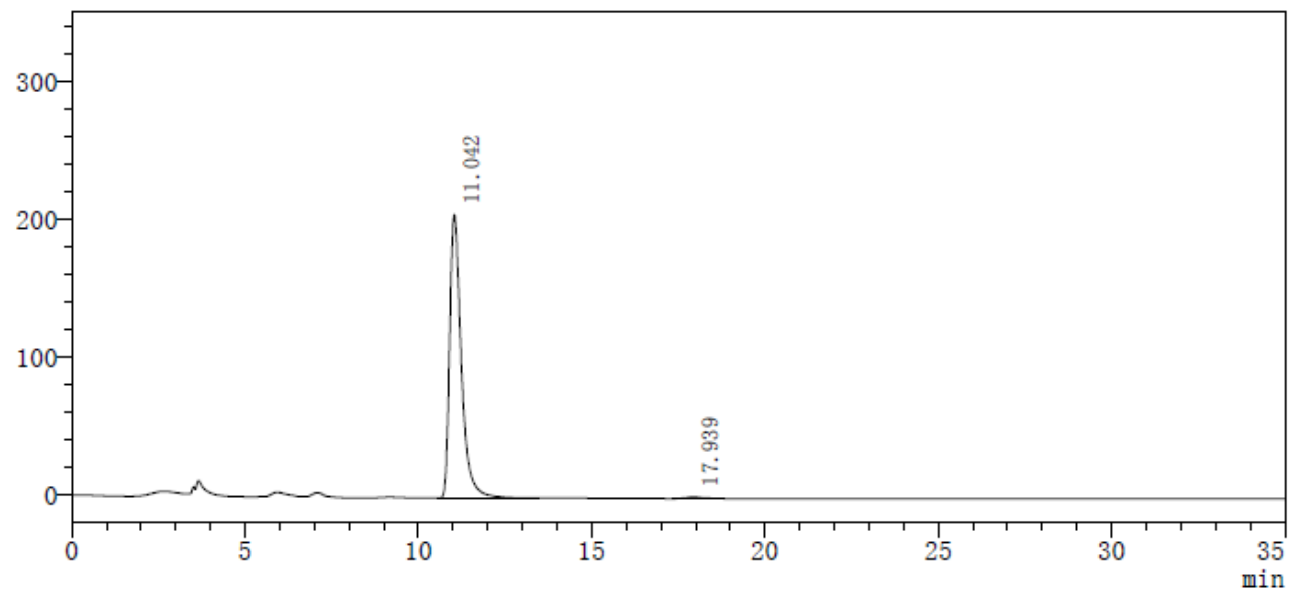

检测器A $220 \mathrm{~nm}$

\begin{tabular}{|c|c|c|c|c|c|}
\hline Peak\# & Time & Area & Height & Height\% & Area\% \\
\hline 1 & 11.042 & 4802471 & 205240 & 99.447 & 99.108 \\
\hline 2 & 17.939 & 43202 & 1141 & 0.553 & 0.892 \\
\hline 总计 & & 4845673 & 206381 & 100.000 & 100.000 \\
\hline
\end{tabular}<smiles>COCC[C@](N)(C(=O)OC)C(C)/C=C/c1ccccc1</smiles>

$(2 S, 3 R)-3 a g$

\section{dimethyl (S)-2-amino-2-((R,E)-4-phenylbut-3-en-2-yl)succinate}

Prepared according to General Procedure B using Pd-6 and $(S, S p)$-L2-Cu, Purification by flash chromatography (PE/EA $=5: 1$ to $1: 2)$ afforded the product as a pale yellow solid, $58 \mathrm{mg}, 92 \%$ yield, 
m.p. $54-56^{\circ} \mathrm{C},>20: 1 \mathrm{dr},>99 \%$ ee, $\mathrm{R}_{\mathrm{f}}=0.4(\mathrm{PE} / \mathrm{EA}, 1: 2)$.

$[\alpha]^{25} \mathrm{D}=47.1\left(\mathrm{c} 1.0, \mathrm{CHCl}_{3}\right)$

${ }^{1} \mathbf{H}$ NMR $\left(400 \mathrm{MHz}, \mathbf{C D C l}_{3}\right) \delta 7.40-7.35(\mathrm{~m}, 2 \mathrm{H}), 7.30(\mathrm{t}, J=7.5 \mathrm{~Hz}, 2 \mathrm{H}), 7.24-7.18(\mathrm{~m}, 1 \mathrm{H})$, $6.45(\mathrm{~d}, J=15.8 \mathrm{~Hz}, 1 \mathrm{H}), 6.13(\mathrm{dd}, J=15.8,9.4 \mathrm{~Hz}, 1 \mathrm{H}), 3.74(\mathrm{~s}, 3 \mathrm{H}), 3.65(\mathrm{~s}, 3 \mathrm{H}), 2.97$ (d, $J=16.7$ $\mathrm{Hz}, 1 \mathrm{H}), 2.65-2.51(\mathrm{~m}, 2 \mathrm{H}), 2.04(\mathrm{~s}, 2 \mathrm{H}), 1.07(\mathrm{~d}, J=6.9 \mathrm{~Hz}, 3 \mathrm{H})$.

${ }^{13} \mathbf{C}$ NMR (101 MHz, $\left.\mathbf{C D C l}_{3}\right) \delta 175.29,171.12,135.89,131.30,128.65,127.52,126.47,125.25$, $60.99,51.33,50.72,44.83,41.36,14.41$.

HRMS (ESI) calcd. for $\mathrm{C}_{16} \mathrm{H}_{22} \mathrm{NO}_{4}{ }^{+}(\mathrm{M}+\mathrm{H})^{+}:$292.1549, Found: 292.1545

the ee value was $>99 \%, \mathrm{t}_{\mathrm{r}}($ minor $)=17.309 \mathrm{~min}, \mathrm{t}_{\mathrm{r}}($ major $)=25.751 \mathrm{~min}($ Chiralcel $\mathrm{IC}, \lambda=220 \mathrm{~nm}$, hexanes : ${ }^{i} \mathrm{PrOH}=90: 10$, flow rate $=1.0 \mathrm{~mL} / \mathrm{min}$ ).

$\mathrm{mV}$

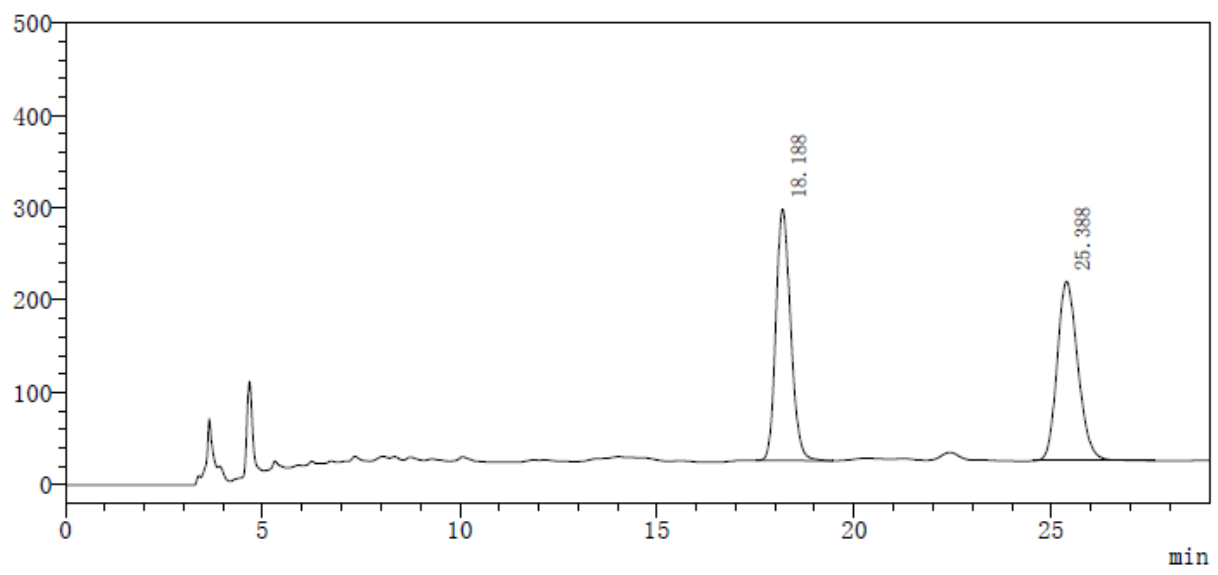

检测器A $220 \mathrm{~nm}$
\begin{tabular}{|c|c|c|c|c|c|}
\hline Peak\# & Time & Area & Height & Height $\%$ & Area\% \\
\hline 1 & 18.188 & 7085351 & 272002 & 58.353 & 49.873 \\
\hline 2 & 25.388 & 7121395 & 194131 & 41.647 & 50.127 \\
\hline 总计 & & 14206745 & 466132 & 100.000 & 100.000 \\
\hline
\end{tabular}

$\mathrm{mV}$

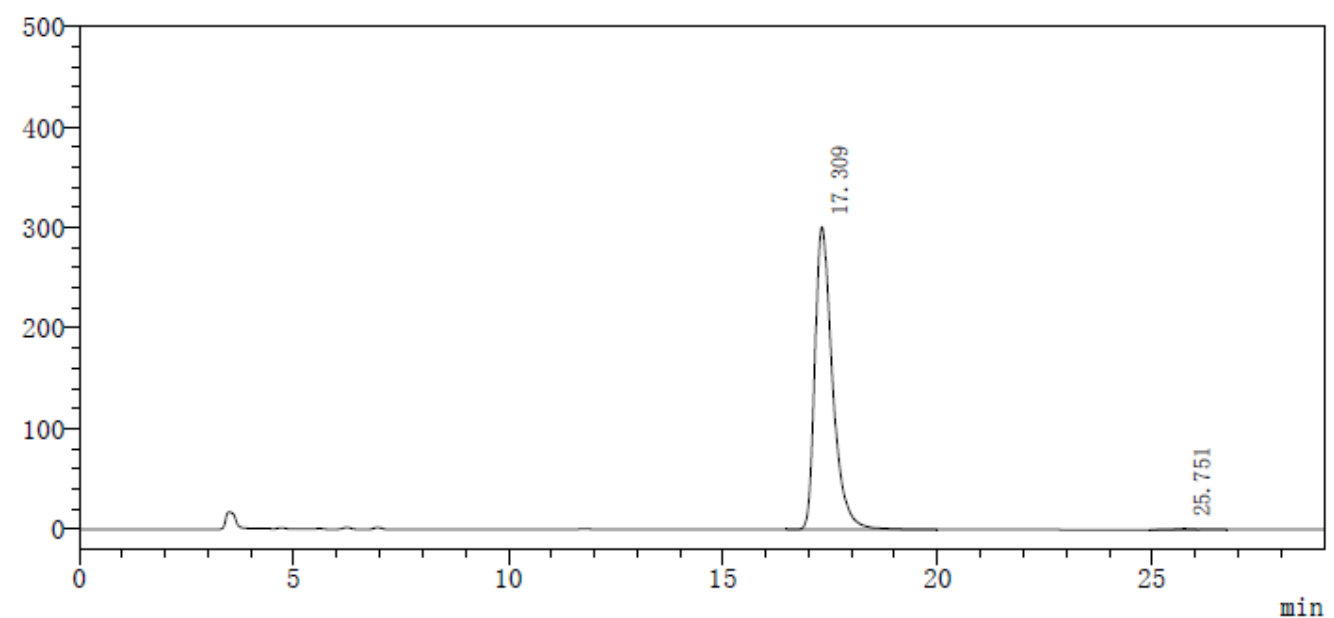

检测器A $220 \mathrm{~nm}$

\begin{tabular}{|c|c|c|c|c|c|}
\hline Peak\# & Time & Area & Height & Height\% & Area\% \\
\hline 1 & 17.309 & 8579021 & 300542 & 99.668 & 99.519 \\
\hline 2 & 25.751 & 41435 & 1001 & 0.332 & 0.481 \\
\hline 总计 & & 8620456 & 301543 & 100.000 & 100.000 \\
\hline
\end{tabular}


<smiles>CC(=O)NCCC(N)(CCNC=O)C(C)C=Cc1ccccc1</smiles>

$(2 S, 3 R)-3 a h$

methyl $(2 S, 3 R, E)-2-a m i n o-2-(2-(((b e n z y l o x y)$ carbonyl)amino)ethyl)-3-methyl-5-phenylpent-4-eno ate

Prepared according to General Procedure B using Pd-6 and $(S, S p)$-L2-Cu, Purification by flash chromatography $(\mathrm{PE} / \mathrm{EA}=5: 1$ to $1: 3)$ afforded the product as a pale yellow oil, $70.5 \mathrm{mg}, 89 \%$ yield, $>20: 1 \mathrm{dr},>99 \%$ ee, $\mathrm{R}_{\mathrm{f}}=0.3(\mathrm{PE} / \mathrm{EA}, 1: 2)$.

$[\alpha]^{25} \mathrm{D}=59.9\left(\mathrm{c} 1.0, \mathrm{CHCl}_{3}\right)$

${ }^{1} \mathbf{H}$ NMR (400 MHz, $\left.\mathbf{C D C l}_{3}\right) \delta 7.44-7.26(\mathrm{~m}, 9 \mathrm{H}), 7.25-7.16(\mathrm{~m}, 1 \mathrm{H}), 6.44(\mathrm{~d}, J=15.8 \mathrm{~Hz}, 1 \mathrm{H}), 6.09$ (dd, $J=15.8,9.0 \mathrm{~Hz}, 1 \mathrm{H}), 5.54(\mathrm{t}, J=5.8 \mathrm{~Hz}, 1 \mathrm{H}), 5.06(\mathrm{~s}, 2 \mathrm{H}), 3.70(\mathrm{~s}, 3 \mathrm{H}), 3.32(\mathrm{dt}, J=13.8,6.9 \mathrm{~Hz}$, 1H), $3.19(\mathrm{dt}, J=13.6,6.2 \mathrm{~Hz}, 1 \mathrm{H}), 2.66(\mathrm{p}, J=7.3 \mathrm{~Hz}, 1 \mathrm{H}), 2.07$ (tt, $J=11.2,6.2 \mathrm{~Hz}, 1 \mathrm{H}), 1.81-1.68(\mathrm{~m}$, $3 \mathrm{H}), 1.00(\mathrm{~d}, J=6.9 \mathrm{~Hz}, 3 \mathrm{H})$.

${ }^{13}$ C NMR (101 MHz, $\left.\mathbf{C D C l}_{3}\right) \delta 176.38,156.31,136.95,136.63,132.55,129.59,128.61,128.50,128.16$, 128.07, 127.54, 126.28, 66.58, 63.39, 52.33, 45.95, 37.98, 37.43, 15.35 .

HRMS (ESI) calcd. for $\mathrm{C}_{23} \mathrm{H}_{29} \mathrm{~N}_{2} \mathrm{O}_{4}{ }^{+}(\mathrm{M}+\mathrm{H})^{+}:$397.2127, Found: 397.2117

the ee value $\mathrm{was}>99 \%, \mathrm{t}_{\mathrm{r}}($ minor $)=29.671 \mathrm{~min}, \mathrm{t}_{\mathrm{r}}($ major $)=47.815 \mathrm{~min}$ (Chiralcel $\mathrm{IC}, \lambda=220 \mathrm{~nm}$, hexanes $:{ }^{i} \mathrm{PrOH}=85: 15$, flow rate $\left.=1.0 \mathrm{~mL} / \mathrm{min}\right)$.

$\mathrm{mV}$

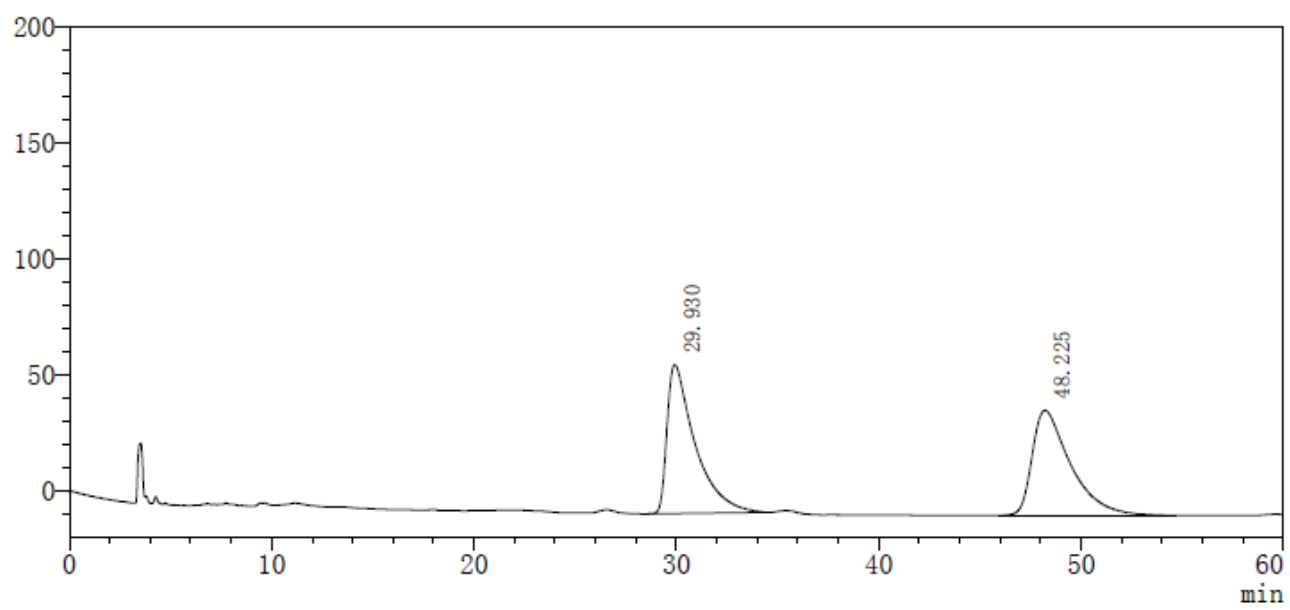

检测器A $220 \mathrm{~nm}$
\begin{tabular}{|c|c|c|c|c|c|}
\hline Peak\# & Time & Area & Height & Height\% & Area\% \\
\hline 1 & 29.930 & 6120226 & 64108 & 58.628 & 50.758 \\
\hline 2 & 48.225 & 5937400 & 45238 & 41.372 & 49.242 \\
\hline 总计 & & 12057626 & 109346 & 100.000 & 100.000 \\
\hline
\end{tabular}




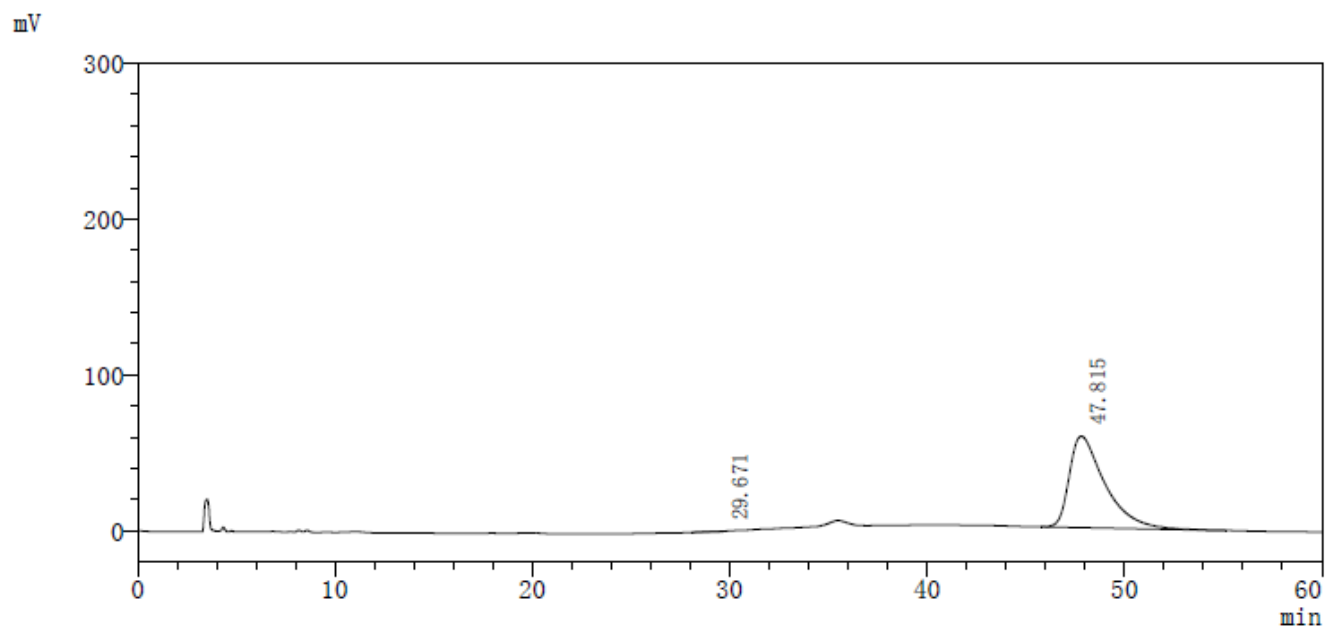

检测器A 220nm
\begin{tabular}{|c|c|c|c|c|c|}
\hline Peak\# & Time & Area & Height & Height $\%$ & Area\% \\
\hline 1 & 29.671 & 8875 & 144 & 0.245 & 0.120 \\
\hline 2 & 47.815 & 7363133 & 58719 & 99.755 & 99.880 \\
\hline 总计 & & 7372008 & 58864 & 100.000 & 100.000 \\
\hline
\end{tabular}<smiles>COC(=O)C(N)(CCSC)C(C)/C=C/c1ccccc1</smiles>

methyl (2S,3R,E)-2-amino-3-methyl-2-(2-(methylthio)ethyl)-5-phenylpent-4-enoate

Prepared according to General Procedure B using Pd-6 and $(S, S p)$-L2-Cu, Purification by flash chromatography (PE/EA $=5: 1$ to $1: 1)$ afforded the product as a pale yellow oil, $55.7 \mathrm{mg}, 95 \%$ yield, $>20: 1 \mathrm{dr},>99 \%$ ee, $\mathrm{R}_{\mathrm{f}}=0.3$ (PE/EA, 1:1).

$[\alpha]^{25} \mathrm{D}=82.3\left(\mathrm{c} 1.0, \mathrm{CHCl}_{3}\right)$

${ }^{1} \mathbf{H}$ NMR $\left(400 \mathrm{MHz}, \mathbf{C D C l}_{3}\right) \delta 7.37(\mathrm{~d}, J=7.6 \mathrm{~Hz}, 2 \mathrm{H}), 7.30(\mathrm{t}, J=7.5 \mathrm{~Hz}, 2 \mathrm{H}), 7.21(\mathrm{t}, J=7.3 \mathrm{~Hz}$, $1 \mathrm{H}), 6.46(\mathrm{~d}, J=15.9 \mathrm{~Hz}, 1 \mathrm{H}), 6.14(\mathrm{dd}, J=15.9,9.1 \mathrm{~Hz}, 1 \mathrm{H}), 3.75(\mathrm{~s}, 3 \mathrm{H}), 2.76-2.62(\mathrm{~m}, 1 \mathrm{H}), 2.55$ $(\mathrm{td}, J=11.8,4.9 \mathrm{~Hz}, 1 \mathrm{H}), 2.33(\mathrm{td}, J=11.8,5.1 \mathrm{~Hz}, 1 \mathrm{H}), 2.15-2.02(\mathrm{~m}, 4 \mathrm{H}), 1.84(\mathrm{ddd}, J=13.8$, $11.0,5.0 \mathrm{~Hz}, 1 \mathrm{H}), 1.60(\mathrm{~s}, 2 \mathrm{H}), 1.03(\mathrm{~d}, J=6.8 \mathrm{~Hz}, 3 \mathrm{H})$.

${ }^{13}$ C NMR (101 MHz, $\left.\mathbf{C D C l}_{3}\right) \delta$ 176.42, 137.09, 132.20, 129.99, 128.56, 127.41, 126.27, 64.03, 52.27, 45.82, 38.30, 29.17, 15.76, 15.66.

HRMS (ESI) calcd. for $\mathrm{C}_{16} \mathrm{H}_{24} \mathrm{NO}_{2} \mathrm{~S}^{+}(\mathrm{M}+\mathrm{H})^{+}: 294.1528$, Found: 294.1525

the ee value $\mathrm{was}>99 \%, \mathrm{t}_{\mathrm{r}}($ minor $)=21.471 \mathrm{~min}, \mathrm{t}_{\mathrm{r}}$ (major) $=37.340 \mathrm{~min}$ (Chiralcel $\mathrm{IC}, \lambda=220 \mathrm{~nm}$, hexanes $:{ }^{i} \mathrm{PrOH}=98: 2$, flow rate $\left.=1.0 \mathrm{~mL} / \mathrm{min}\right)$. 
$\mathrm{mV}$
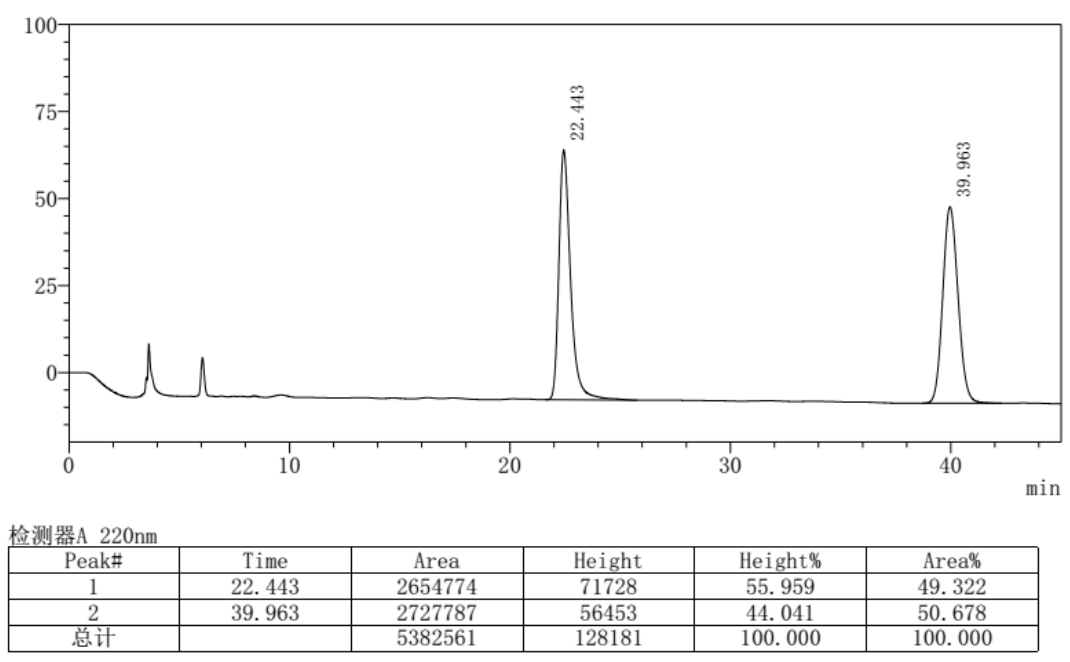

$\mathrm{mV}$

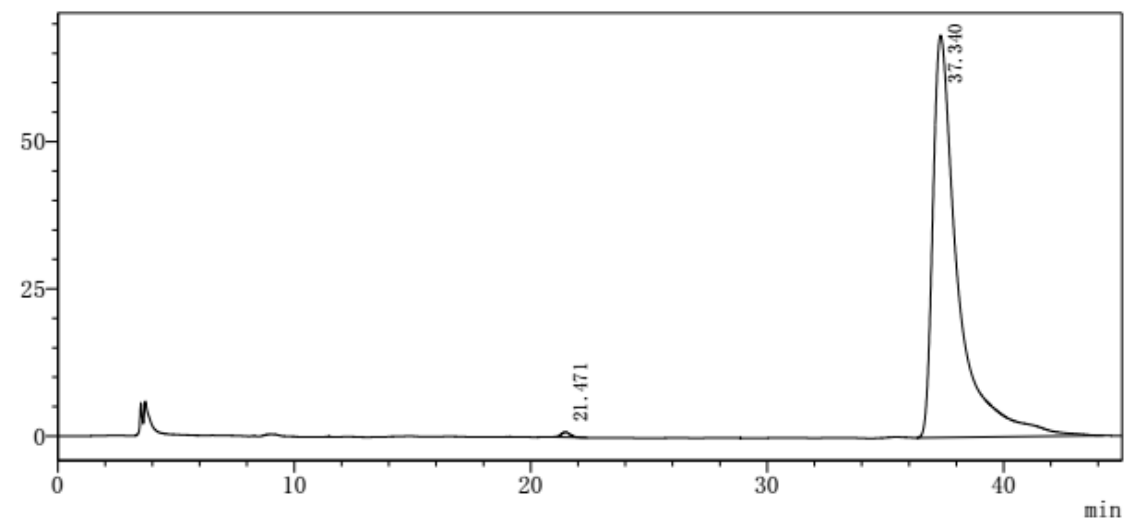

检测器A $220 \mathrm{~nm}$
\begin{tabular}{|c|c|c|c|c|c|}
\hline Peak\# & Time & Area & Height & Height\% & Area\% \\
\hline 1 & 21.471 & 22805 & 870 & 1.258 & 0.451 \\
\hline 2 & 37.340 & 5032214 & 68281 & 98.742 & 99.549 \\
\hline 总计 & & 5055019 & 69151 & 100.000 & 100.000 \\
\hline
\end{tabular}<smiles>C=CC[C@](N)(C(C)=O)C(C)/C=C/c1ccccc1</smiles>

$(2 S, 3 R)-3$ aj

\section{methyl (2S,3R,E)-2-allyl-2-amino-3-methyl-5-phenylpent-4-enoate}

Prepared according to General Procedure B using Pd-6 and $(S, S p)$-L2-Cu, Purification by flash chromatography $(\mathrm{PE} / \mathrm{EA}=5: 1$ to $1: 1)$ afforded the product as a pale yellow oil, $31 \mathrm{mg}, 59 \%$ yield, >20:1 dr, >99\% ee, $\mathrm{R}_{\mathrm{f}}=0.4(\mathrm{PE} / \mathrm{EA}, 1: 1)$.

$[\alpha]^{25} \mathrm{D}=87.8\left(\mathrm{c} 0.5, \mathrm{CHCl}_{3}\right)$

${ }^{1}$ H NMR (400 MHz, CDCl $) \delta 7.42-7.36(\mathrm{~m}, 2 \mathrm{H}), 7.30(\mathrm{t}, J=7.6 \mathrm{~Hz}, 2 \mathrm{H}), 7.24-7.19(\mathrm{~m}, 1 \mathrm{H})$, $6.46(\mathrm{~d}, J=15.9 \mathrm{~Hz}, 1 \mathrm{H}), 6.19(\mathrm{dd}, J=15.9,9.3 \mathrm{~Hz}, 1 \mathrm{H}), 5.72-5.54(\mathrm{~m}, 1 \mathrm{H}), 5.16-5.07(\mathrm{~m}, 2 \mathrm{H})$, $3.75(\mathrm{~s}, 3 \mathrm{H}), 2.70(\mathrm{dq}, J=9.3,6.9 \mathrm{~Hz}, 1 \mathrm{H}), 2.57$ (ddt, $J=13.7,6.3,1.3 \mathrm{~Hz}, 1 \mathrm{H}), 2.25(\mathrm{dd}, J=13.6$, $8.6 \mathrm{~Hz}, 1 \mathrm{H}), 1.62(\mathrm{~s}, 2 \mathrm{H}), 1.04(\mathrm{~d}, J=6.9 \mathrm{~Hz}, 3 \mathrm{H})$. 
${ }^{13}$ C NMR (101 MHz, CDCl $\left.\mathbf{3}\right) \delta 176.93,137.24,132.95,131.86,130.53,128.54,127.31,126.27$, 119.59, 63.90, 52.17, 45.57, 43.15, 16.05 .

HRMS (ESI) calcd. for $\mathrm{C}_{16} \mathrm{H}_{22} \mathrm{NO}_{2}{ }^{+}(\mathrm{M}+\mathrm{H})^{+}:$260.1651, Found: 260.1649

the ee value $\mathrm{was}>99 \%, \mathrm{t}_{\mathrm{r}}($ minor $)=9.718 \mathrm{~min}, \mathrm{t}_{\mathrm{r}}$ (major) $=11.925 \mathrm{~min}$ (Chiralcel $\mathrm{IC}, \lambda=220 \mathrm{~nm}$, hexanes $:{ }^{i} \mathrm{PrOH}=98: 2$, flow rate $=1.0 \mathrm{~mL} / \mathrm{min}$ ).

$\mathrm{mV}$

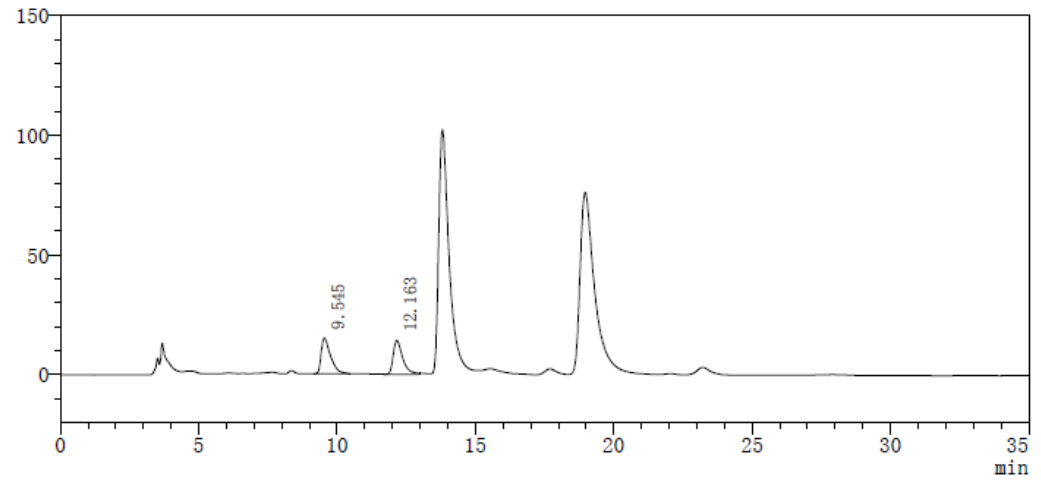

检测器A $220 \mathrm{~nm}$
\begin{tabular}{|c|c|c|c|c|c|}
\hline Peak\# & Time & Area & Height & Height\% & Area\% \\
\hline 1 & 9.545 & 352119 & 14990 & 51.318 & 50.495 \\
\hline 2 & 12.163 & 345222 & 14220 & 48.682 & 49.505 \\
\hline 总计 & & 697341 & 29211 & 100.000 & 100.000 \\
\hline
\end{tabular}

$\mathrm{mV}$

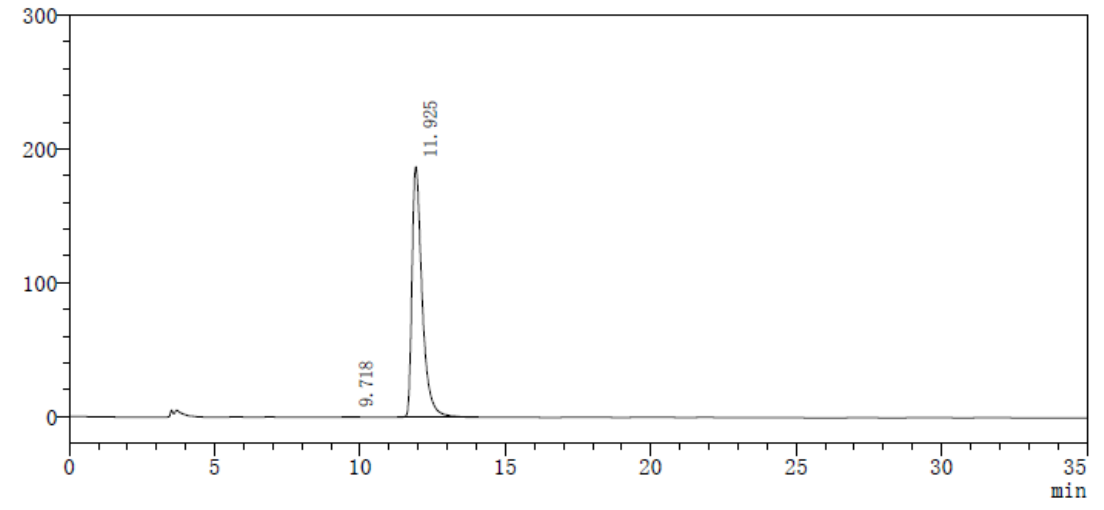

检测器A $220 \mathrm{~nm}$
\begin{tabular}{|c|c|c|c|c|c|}
\hline Peak\# & Time & Area & Height & Height\% & Area\% \\
\hline 1 & 9.718 & 1239 & 73 & 0.039 & 0.027 \\
\hline 2 & 11.925 & 4542063 & 187244 & 99.961 & 99.973 \\
\hline 总计 & & 4543302 & 187317 & 100.000 & 100.000 \\
\hline
\end{tabular}

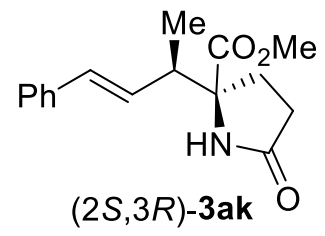

methyl $(S)$-5-oxo-2-((R,E)-4-phenylbut-3-en-2-yl)pyrrolidine-2-carboxylate

Prepared according to General Procedure B using Pd-6 and $(S, S p)$-L2-Cu, Purification by flash chromatography (PE/EA = 5:1 to 1:3) afforded the product as a pale yellow solid, $42 \mathrm{mg}, 76 \%$ yield, 
m.p. $117-118{ }^{\circ} \mathrm{C},>20: 1 \mathrm{dr},>99 \%$ ee, $\mathrm{R}_{\mathrm{f}}=0.4(\mathrm{PE} / \mathrm{EA}, 1: 2)$.

$[\alpha]^{25} \mathrm{D}=56.1\left(\mathrm{c} 1.0, \mathrm{CHCl}_{3}\right)$

${ }^{1}$ H NMR (400 MHz, CDCl $)$ ) $8.38-7.28(\mathrm{~m}, 4 \mathrm{H}), 7.26-7.19(\mathrm{~m}, 1 \mathrm{H}), 7.15(\mathrm{~s}, 1 \mathrm{H}), 6.53(\mathrm{~d}, J=$ $15.8 \mathrm{~Hz}, 1 \mathrm{H}), 6.01(\mathrm{dd}, J=15.8,9.4 \mathrm{~Hz}, 1 \mathrm{H}), 3.78(\mathrm{~s}, 3 \mathrm{H}), 2.81(\mathrm{dq}, J=9.5,6.9 \mathrm{~Hz}, 1 \mathrm{H}), 2.45-2.35$ $(\mathrm{m}, 1 \mathrm{H}), 2.34-2.29(\mathrm{~m}, 2 \mathrm{H}), 2.24(\mathrm{dt}, J=14.5,6.0 \mathrm{~Hz}, 1 \mathrm{H}), 1.10(\mathrm{~d}, J=6.9 \mathrm{~Hz}, 3 \mathrm{H})$.

${ }^{13} \mathbf{C}$ NMR (101 MHz, $\left.\mathbf{C D C l}_{3}\right) \delta$ 176.65, 172.84, 135.64, 132.14, 127.61, 127.24, 126.72, 125.31, $67.86,51.62,44.43,28.85,27.74,14.82$.

HRMS (ESI) calcd. for $\mathrm{C}_{16} \mathrm{H}_{20} \mathrm{NO}_{3}{ }^{+}(\mathrm{M}+\mathrm{H})^{+}: 274.1443$, Found: 274.1436

the ee value $\mathrm{was}>99 \%, \mathrm{t}_{\mathrm{r}}$ (major) $=49.085 \mathrm{~min}, \mathrm{t}_{\mathrm{r}}$ (minor) $=56.569 \mathrm{~min}$ (Chiralc el $\mathrm{IC}, \lambda=220 \mathrm{~nm}$, hexanes : ${ }^{i} \mathrm{PrOH}=85: 15$, flow rate $=1.0 \mathrm{~mL} / \mathrm{min}$ ).

$\mathrm{mV}$

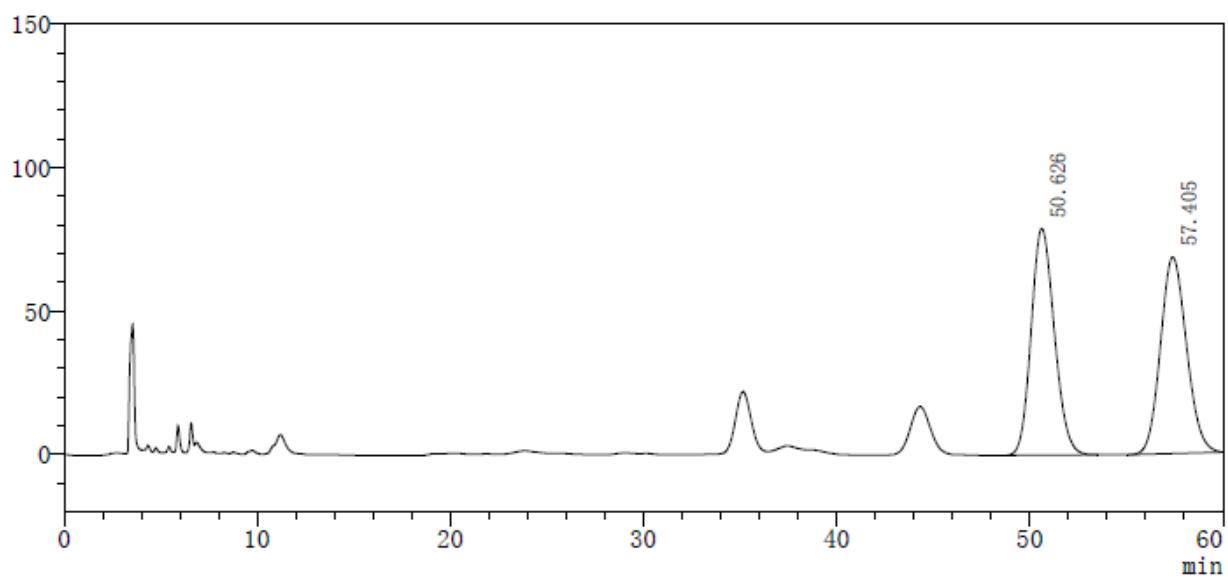

检测器A 220nm
\begin{tabular}{|c|c|c|c|c|c|}
\hline Peak\# & Time & Area & Height & Height\% & Area\% \\
\hline 1 & 50.626 & 6619617 & 79013 & 53.586 & 50.694 \\
\hline 2 & 57.405 & 6438309 & 68438 & 46.414 & 49.306 \\
\hline 总计 & & 13057926 & 147451 & 100.000 & 100.000 \\
\hline
\end{tabular}

$\mathrm{mV}$

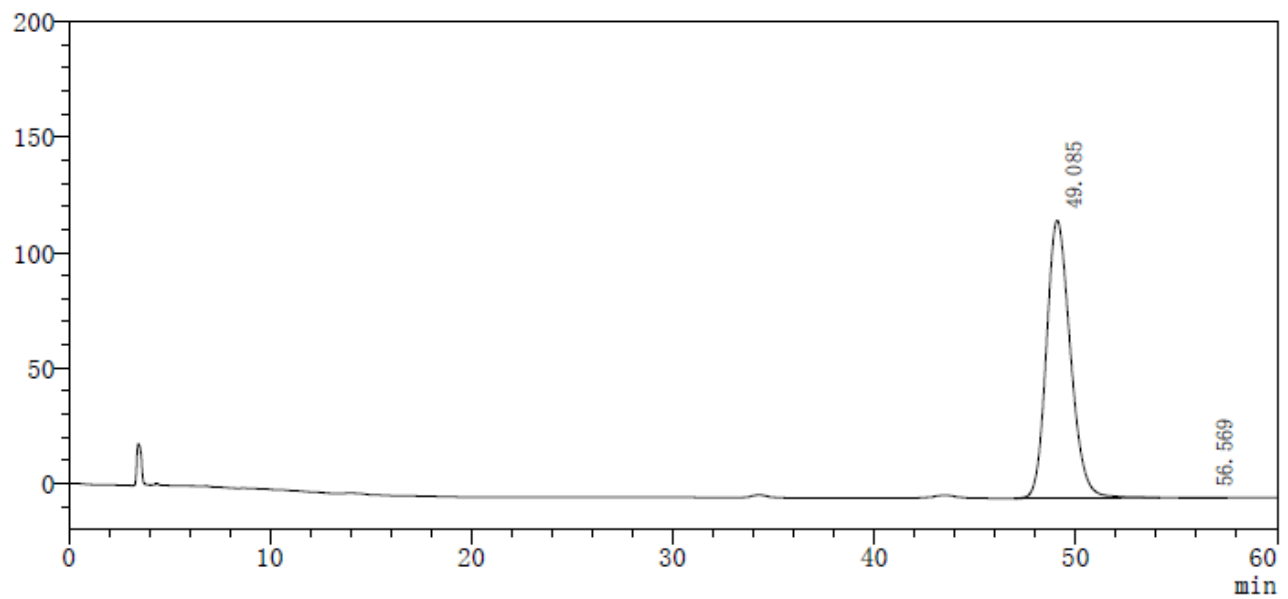

检测器A $220 \mathrm{~nm}$
\begin{tabular}{|c|c|c|c|c|c|}
\hline Peak\# & Time & Area & Height & Height $\%$ & Area\% \\
\hline 1 & 49.085 & 9943508 & 120373 & 99.890 & 99.848 \\
\hline 2 & 56.569 & 15135 & 133 & 0.110 & 0.152 \\
\hline 总计 & & 9958643 & 120506 & 100.000 & 100.000 \\
\hline
\end{tabular}


<smiles>[Y6]C(/C=C/c1ccccc1)C1(N)CCOC1=O</smiles>

$(2 S, 3 R)-3$ al

\section{(R)-3-amino-3-((R,E)-4-phenylbut-3-en-2-yl)dihydrofuran-2(3H)-one}

Prepared according to General Procedure B using Pd-6 and $(S, S p)$-L2-Cu, Purification by flash chromatography $(\mathrm{PE} / \mathrm{EA}=5: 1$ to $1: 3)$ afforded the product as a pale yellow oil, $31 \mathrm{mg}, 67 \%$ yield, $>20: 1 \mathrm{dr},>99 \%$ ee, $\mathrm{R}_{\mathrm{f}}=0.3$ (PE/EA, 1:2).

$[\alpha]^{25} \mathrm{D}=57.7\left(\mathrm{c} 1.0, \mathrm{CHCl}_{3}\right)$

${ }^{1}$ H NMR (400 MHz, $\left.\mathbf{C D C l}_{3}\right) \delta 7.41-7.36(\mathrm{~m}, 2 \mathrm{H}), 7.35$ - $7.29(\mathrm{~m}, 2 \mathrm{H}), 7.26-7.21(\mathrm{~m}, 1 \mathrm{H}), 6.54(\mathrm{~d}$, $J=15.8 \mathrm{~Hz}, 1 \mathrm{H}), 6.14(\mathrm{dd}, J=15.8,8.6 \mathrm{~Hz}, 1 \mathrm{H}), 4.37(\mathrm{td}, J=8.6,6.4 \mathrm{~Hz}, 1 \mathrm{H}), 4.24(\mathrm{td}, J=8.7,5.7$ $\mathrm{Hz}, 1 \mathrm{H}), 2.74-2.60(\mathrm{~m}, 1 \mathrm{H}), 2.45(\mathrm{ddd}, J=13.2,8.3,6.4 \mathrm{~Hz}, 1 \mathrm{H}), 2.00(\mathrm{ddd}, J=13.7,8.3,5.7 \mathrm{~Hz}$, $1 \mathrm{H}), 1.63(\mathrm{~s}, 2 \mathrm{H}), 1.17(\mathrm{~d}, J=6.7 \mathrm{~Hz}, 3 \mathrm{H})$.

${ }^{13}$ C NMR (101 MHz, $\left.\mathbf{C D C l}_{3}\right) \delta$ 179.77, 136.74, 133.02, 128.92, 128.65, 127.71, 126.31, 65.31, 60.71, 45.01, 32.33, 15.57 .

HRMS (ESI) calcd. for $\mathrm{C}_{14} \mathrm{H}_{18} \mathrm{NO}_{2}{ }^{+}(\mathrm{M}+\mathrm{H})^{+}: 232.1338$, Found: 232.1335

the ee value $\mathrm{was}>99 \%, \mathrm{t}_{\mathrm{r}}($ minor $)=25.385 \mathrm{~min}, \mathrm{t}_{\mathrm{r}}($ major $)=34.215 \mathrm{~min}$ (Chiralcel $\mathrm{IC}, \lambda=220 \mathrm{~nm}$, hexanes $:{ }^{i} \mathrm{PrOH}=85: 15$, flow rate $\left.=1.0 \mathrm{~mL} / \mathrm{min}\right)$.

$\mathrm{mV}$

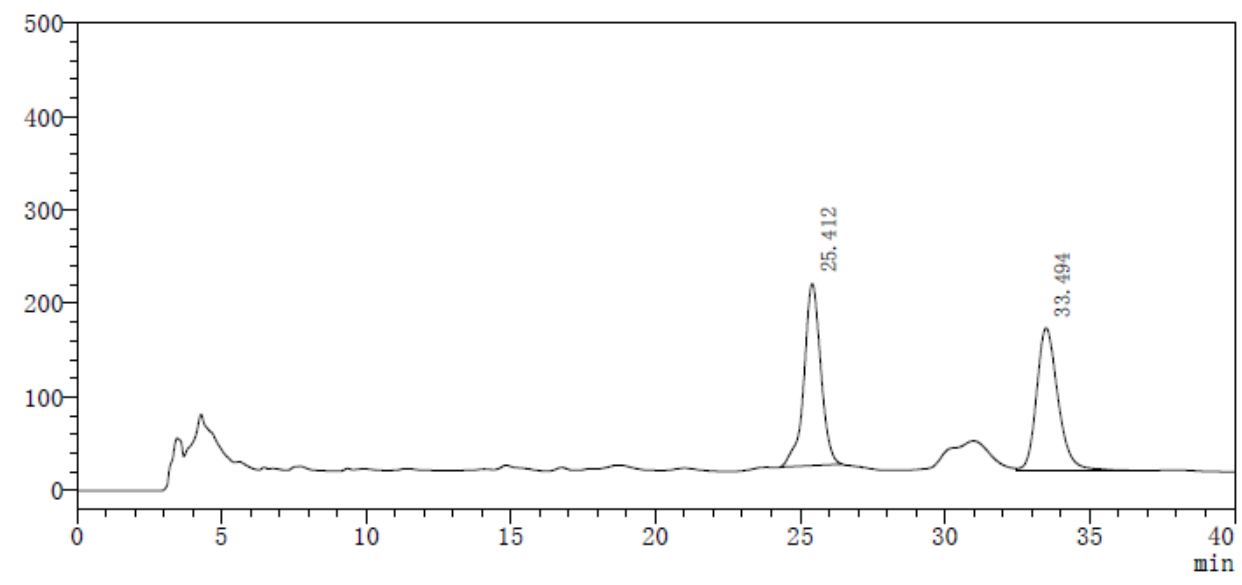

检测器A 220 nm
\begin{tabular}{|c|c|c|c|c|c|}
\hline Peak\# & Time & Area & Height & Height\% & Area\% \\
\hline 1 & 25.412 & 7867502 & 194439 & 56.044 & 49.972 \\
\hline 2 & 33.494 & 7876453 & 152503 & 43.956 & 50.028 \\
\hline 总计 & & 15743955 & 346942 & 100.000 & 100.000 \\
\hline
\end{tabular}


$\mathrm{mV}$

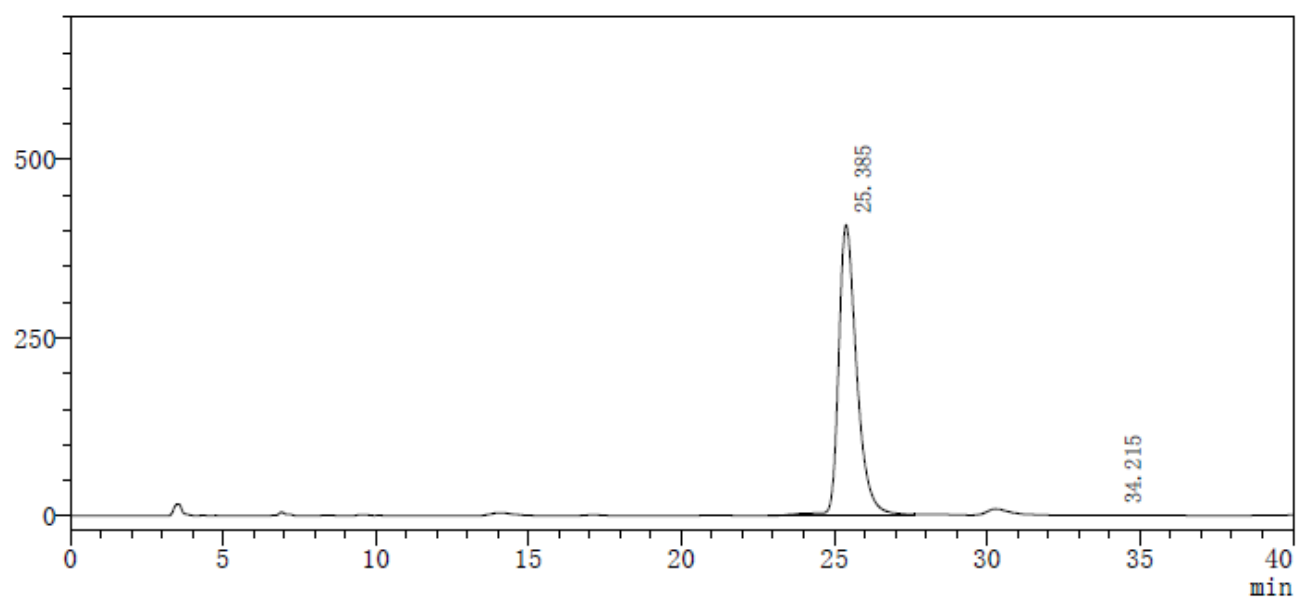

检测器A $220 \mathrm{~nm}$
\begin{tabular}{|c|c|c|c|c|c|}
\hline Peak\# & Time & Area & Height & Height\% & Area\% \\
\hline 1 & 25.385 & 16818373 & 407375 & 99.940 & 99.937 \\
\hline 2 & 34.215 & 10621 & 246 & 0.060 & 0.063 \\
\hline 总计 & & 16828994 & 407621 & 100.000 & 100.000 \\
\hline
\end{tabular}<smiles>COC1(C(C)/C=C/c2ccccc2)CCC(c2ccccc2)=N1</smiles>

methyl $(S)$-5-phenyl-2-((R,E)-4-phenylbut-3-en-2-yl)-3,4-dihydro-2H-pyrrole-2-carboxylate

Prepared according to General Procedure B using Pd-6 and $(S, S p)-\mathbf{L 2}-\mathbf{C u}$, Purification by flash chromatography $(\mathrm{PE} / \mathrm{EA}=30: 1$ to $15: 1)$ afforded the product as a pale yellow oil, $60 \mathrm{mg}, 90 \%$ yield, >20:1 dr, >99\% ee, $\mathrm{R}_{\mathrm{f}}=0.3$ (PE/EA, 15:1).

$[\alpha]^{25} \mathrm{D}=-49.7\left(\mathrm{c} 1.0, \mathrm{CHCl}_{3}\right)$

${ }^{1} \mathbf{H}$ NMR $\left(400 \mathrm{MHz}, \mathbf{C D C l}_{3}\right) \delta 8.00-7.86(\mathrm{~m}, 2 \mathrm{H}), 7.49-7.37(\mathrm{~m}, 3 \mathrm{H}), 7.31-7.24(\mathrm{~m}, 4 \mathrm{H}), 7.21-$ $7.15(\mathrm{~m}, 1 \mathrm{H}), 6.49(\mathrm{~d}, J=15.8 \mathrm{~Hz}, 1 \mathrm{H}), 5.96(\mathrm{dd}, J=15.8,8.9 \mathrm{~Hz}, 1 \mathrm{H}), 3.75(\mathrm{~s}, 3 \mathrm{H}), 3.34-3.21(\mathrm{~m}$, 1H), $3.11-2.97$ (m, 1H), 2.88 (ddd, $J=17.3,10.0,5.6 \mathrm{~Hz}, 1 \mathrm{H}), 2.41$ (ddd, $J=13.5,10.3,5.6 \mathrm{~Hz}, 1 \mathrm{H}$ ), 2.14 (ddd, $J=13.5,10.1,6.1 \mathrm{~Hz}, 1 \mathrm{H}), 1.18(\mathrm{~d}, J=6.8 \mathrm{~Hz}, 3 \mathrm{H})$.

${ }^{13}$ C NMR (101 MHz, CDCl $) \delta 174.98,174.80,137.35,134.05,131.67,130.94,130.42,128.55$, $128.47,128.13,127.32,126.24,87.14,52.43,44.89,35.85,28.65,15.70$.

HRMS (ESI) calcd. for $\mathrm{C}_{22} \mathrm{H}_{24} \mathrm{NO}_{2}{ }^{+}(\mathrm{M}+\mathrm{H})^{+}: 334.1807$, Found: 334.1800

the ee value was $>99 \%, \mathrm{t}_{\mathrm{r}}($ minor $)=12.429 \mathrm{~min}, \mathrm{t}_{\mathrm{r}}($ major $)=17.027 \mathrm{~min}($ Chiralcel $\mathrm{IC}, \lambda=220 \mathrm{~nm}$, hexanes $:{ }^{i} \mathrm{PrOH}=98: 2$, flow rate $\left.=1.0 \mathrm{~mL} / \mathrm{min}\right)$. 
$\mathrm{mV}$

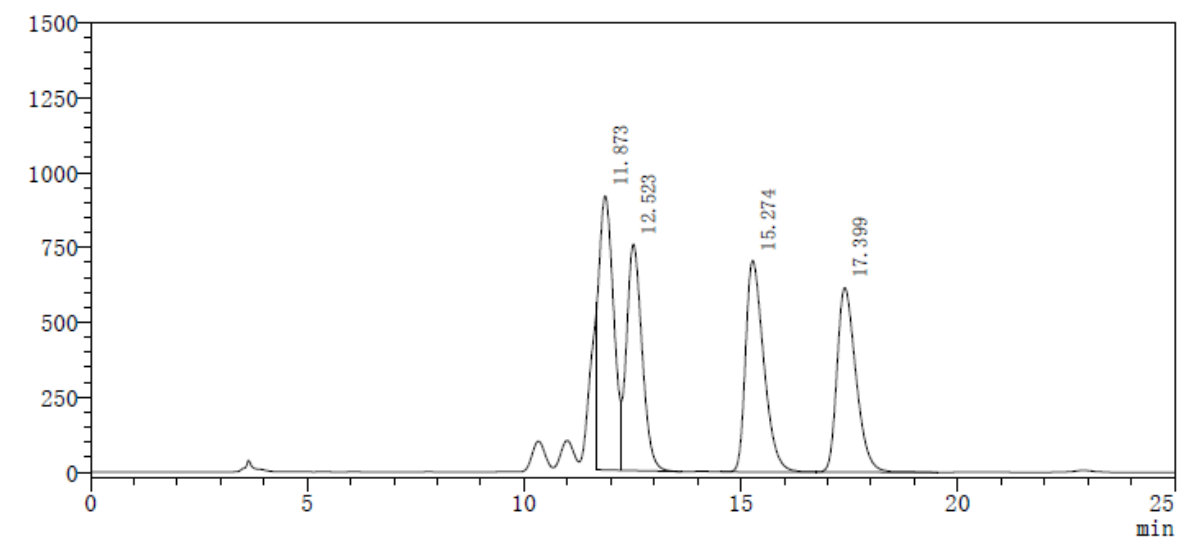

检测器A 220nm
\begin{tabular}{|c|c|c|c|c|c|}
\hline Peak\# & Time & Area & Height & Height\% & Area\% \\
\hline 1 & 11.873 & 21204054 & 915866 & 30.589 & 26.639 \\
\hline 2 & 12.523 & 18940623 & 755935 & 25.248 & 23.795 \\
\hline 3 & 15.274 & 20648268 & 706281 & 23.589 & 25.941 \\
\hline 4 & 17.399 & 18804905 & 615985 & 20.574 & 23.625 \\
\hline 总计 & & 79597850 & 2994067 & 100.000 & 100.000 \\
\hline
\end{tabular}

$\mathrm{mV}$

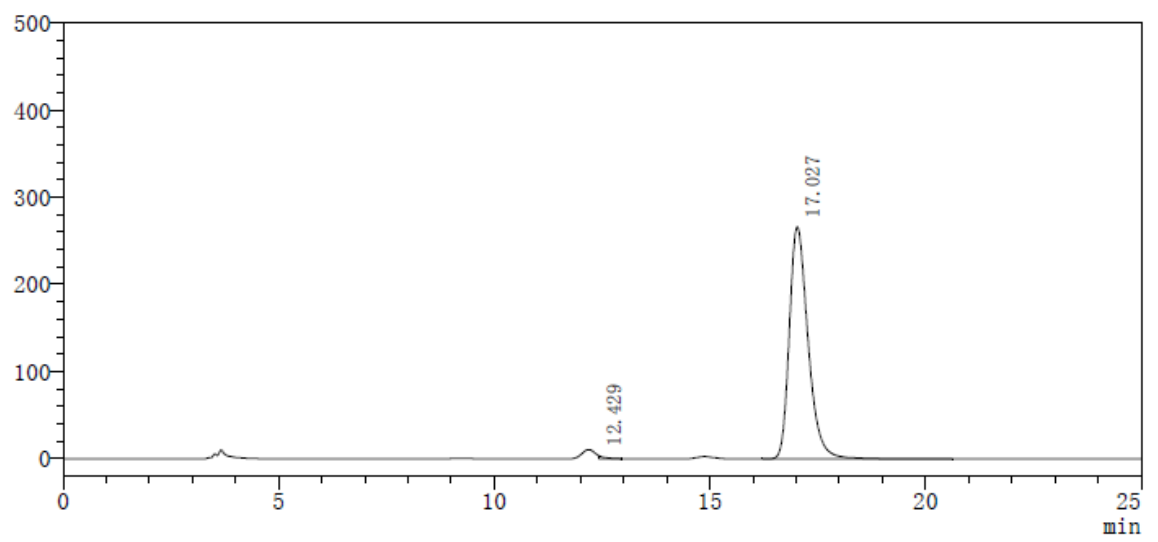

检测器A 220nm
\begin{tabular}{|c|c|c|c|c|c|}
\hline Peak\# & Time & Area & Height & Height\% & Area\% \\
\hline 1 & 12.429 & 32084 & 3303 & 1.226 & 0.398 \\
\hline 2 & 17.027 & 8022217 & 266073 & 98.774 & 99.602 \\
\hline 总计 & & 8054300 & 269376 & 100.000 & 100.000 \\
\hline
\end{tabular}<smiles>COC1(C(C)/C=C/c2ccccc2)CC(c2ccccc2)=NC1(C)OCCO</smiles>

(R)-methyl 5-phenyl-2-((R,E)-4-phenylbut-3-en-2-yl)-3,4-dihydro-2H-pyrrole-2-carboxylate

Prepared according to General Procedure B using Pd-6 and $(S, S p)$-L2-Cu, Purification by flash chromatography (PE/EA = 30:1 to 15:1) afforded the product as a pale yellow oil, $62 \mathrm{mg}, 93 \%$ yield, $13: 1 \mathrm{dr},>99 \%$ ee, $\mathrm{R}_{\mathrm{f}}=0.3$ (PE/EA, 15:1). $[\alpha]^{25} \mathrm{D}=107.2\left(\mathrm{c} 1.0, \mathrm{CHCl}_{3}\right)$

${ }^{1}$ H NMR (400 MHz, CDCl $\left.)_{3}\right) \delta 7.89-7.83(\mathrm{~m}, 2 \mathrm{H}), 7.46-7.36(\mathrm{~m}, 3 \mathrm{H}), 7.31-7.21(\mathrm{~m}, 4 \mathrm{H}), 7.16$ (t, $J=7.1 \mathrm{~Hz}, 1 \mathrm{H}), 6.44(\mathrm{~d}, J=16.0 \mathrm{~Hz}, 1 \mathrm{H}), 6.22(\mathrm{dd}, J=16.0,7.5 \mathrm{~Hz}, 1 \mathrm{H}), 3.74(\mathrm{~s}, 3 \mathrm{H}), 3.25(\mathrm{p}, J=$ 
$6.9 \mathrm{~Hz}, 1 \mathrm{H}), 3.11-2.97(\mathrm{~m}, 1 \mathrm{H}), 2.93-2.81(\mathrm{~m}, 1 \mathrm{H}), 2.57-2.45(\mathrm{~m}, 1 \mathrm{H}), 2.09-1.95(\mathrm{~m}, 1 \mathrm{H}), 1.13(\mathrm{~d}$, $J=6.8 \mathrm{~Hz}, 3 \mathrm{H})$.

${ }^{13}$ C NMR (101 MHz, CDCl 3$) \delta 174.53,174.35,137.52,134.09,131.36,130.86,130.58,128.48$, $128.43,128.08,127.16,126.24,87.83,52.41,43.98,35.94,27.52,15.01$.

HRMS (ESI) calcd. for $\mathrm{C}_{22} \mathrm{H}_{24} \mathrm{NO}_{2}{ }^{+}(\mathrm{M}+\mathrm{H})^{+}: 334.1807$, Found: 334.1797

the ee value $\mathrm{was}>99 \%, \mathrm{t}_{\mathrm{r}}($ major $)=11.864 \mathrm{~min}, \mathrm{t}_{\mathrm{r}}($ minor $)=15.496 \mathrm{~min}$ (Chiralcel $\mathrm{IC}, \lambda=220 \mathrm{~nm}$, hexanes $:{ }^{i} \mathrm{PrOH}=98: 2$, flow rate $=1.0 \mathrm{~mL} / \mathrm{min}$ ).

$\mathrm{mV}$

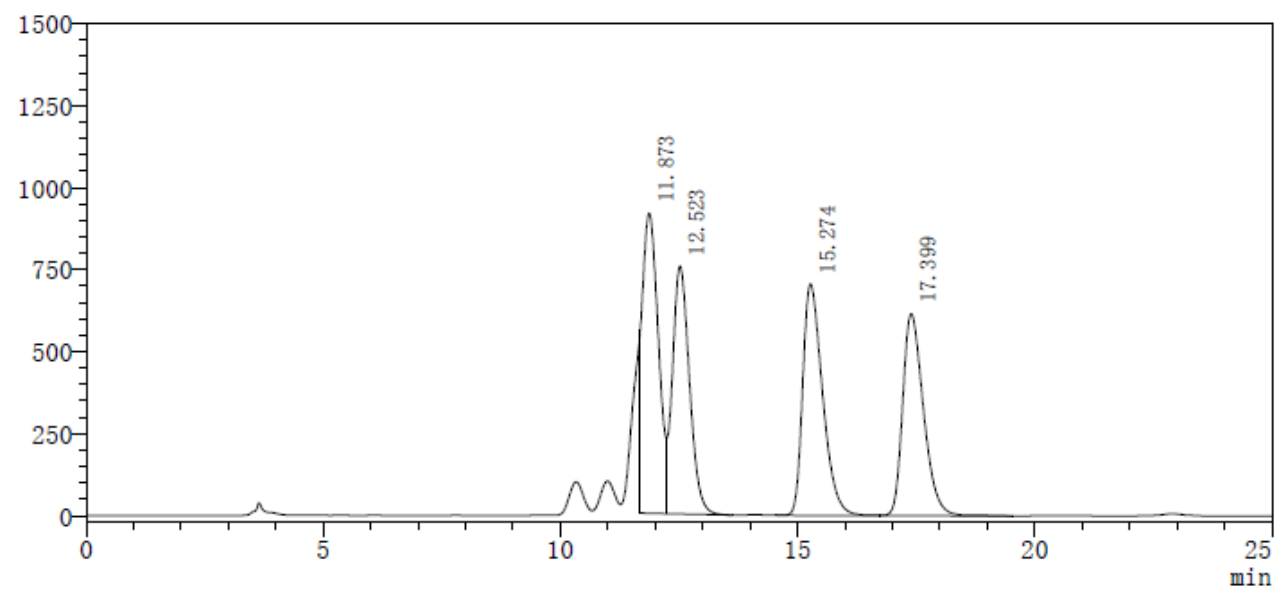

检测器A $220 \mathrm{~nm}$
\begin{tabular}{|c|c|c|c|c|c|}
\hline Peak\# & Time & Area & Height & Height\% & Area\% \\
\hline 1 & 11.873 & 21204054 & 915866 & 30.589 & 26.639 \\
\hline 2 & 12.523 & 18940623 & 755935 & 25.248 & 23.795 \\
\hline 3 & 15.274 & 20648268 & 706281 & 23.589 & 25.941 \\
\hline 4 & 17.399 & 18804905 & 615985 & 20.574 & 23.625 \\
\hline 总计 & & 79597850 & 2994067 & 100.000 & 100.000 \\
\hline
\end{tabular}

$\mathrm{mV}$

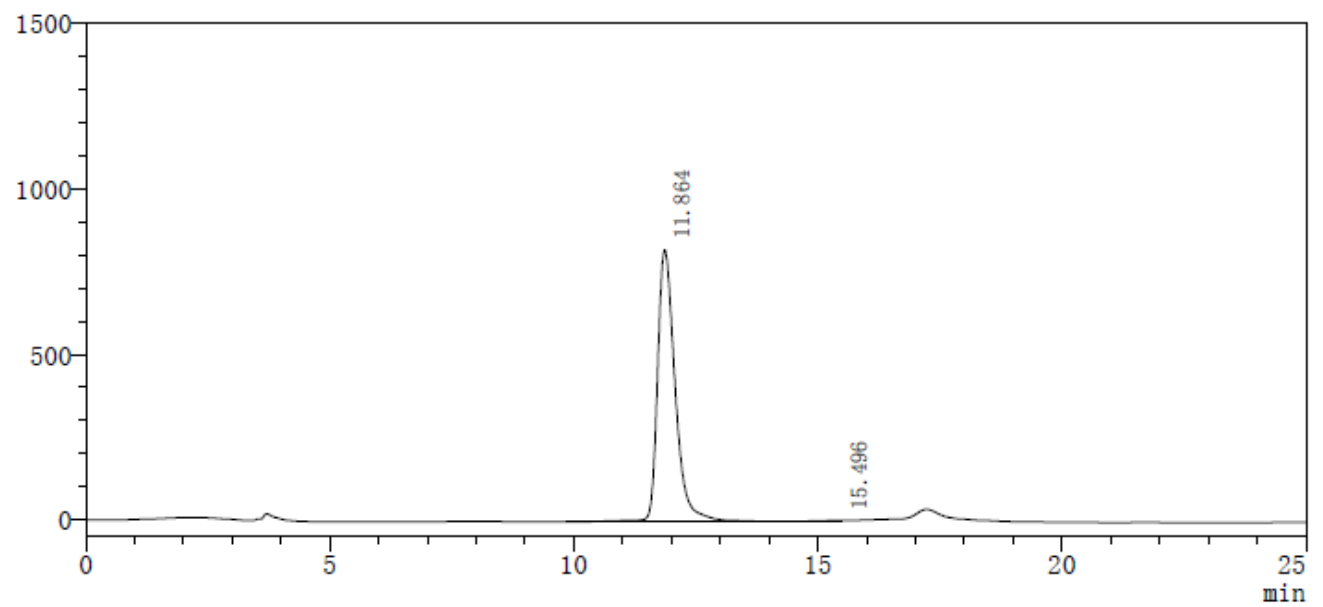

检测器A $220 \mathrm{~nm}$
\begin{tabular}{|c|c|c|c|c|c|}
\hline Peak\# & Time & Area & Height & Height\% & Area\% \\
\hline 1 & 11.864 & 20857975 & 821276 & 99.650 & 99.617 \\
\hline 2 & 15.496 & 80283 & 2886 & 0.350 & 0.383 \\
\hline 总计 & & 20938259 & 824162 & 100.000 & 100.000 \\
\hline
\end{tabular}


<smiles>CCOC(=O)C(C)C(C)C=Cc1ccccc1</smiles>

$(2 S, 3 R)-3$ an

\section{ethyl $(2 S, 3 R, E)$-2-amino-2,3-dimethyl-5-phenylpent-4-enoate}

Prepared according to General Procedure B using Pd-6 and $(S, S p)$-L2-Cu, Purification by flash chromatography $(\mathrm{PE} / \mathrm{EA}=5: 1$ to 1:1) afforded the product as a pale yellow oil, $38 \mathrm{mg}, 77 \%$ yield, >20:1 dr, >99\% ee, $\mathrm{R}_{\mathrm{f}}=0.4(\mathrm{PE} / \mathrm{EA}, 1: 1)$.

$[\alpha]^{25} \mathrm{D}=63.2\left(\mathrm{c} 1.0, \mathrm{CHCl}_{3}\right)$

${ }^{1}$ H NMR (400 MHz, $\left.\mathbf{C D C l}_{3}\right) \delta 7.44-7.35(\mathrm{~m}, 2 \mathrm{H}), 7.30(\mathrm{dd}, J=8.5,6.8 \mathrm{~Hz}, 2 \mathrm{H}), 7.24-7.16(\mathrm{~m}$, $1 \mathrm{H}), 6.45(\mathrm{~d}, J=15.9 \mathrm{~Hz}, 1 \mathrm{H}), 6.18(\mathrm{dd}, J=15.8,9.1 \mathrm{~Hz}, 1 \mathrm{H}), 4.19(\mathrm{q}, J=7.1 \mathrm{~Hz}, 2 \mathrm{H}), 2.65$ (dq, $J=$ 9.2, 6.9 Hz, 1H), $1.62(\mathrm{~s}, 2 \mathrm{H}), 1.31-1.25(\mathrm{~m}, 6 \mathrm{H}), 1.06(\mathrm{~d}, J=6.9 \mathrm{~Hz}, 3 \mathrm{H})$.

${ }^{13} \mathbf{C}$ NMR (101 MHz, $\left.\mathbf{C D C l}_{3}\right) \delta 177.31,137.33,131.76,130.72,128.52,127.24,126.22,61.05,60.48$, $45.84,24.86,15.69,14.31$.

HRMS (ESI) calcd. for $\mathrm{C}_{15} \mathrm{H}_{22} \mathrm{NO}_{2}{ }^{+}(\mathrm{M}+\mathrm{H})^{+}:$:248.1651, Found: 248.1647

the ee value $\mathrm{was}>99 \%, \mathrm{t}_{\mathrm{r}}($ minor $)=16.542 \mathrm{~min}, \mathrm{t}_{\mathrm{r}}($ major $)=20.991 \mathrm{~min}($ Chiralcel $\mathrm{IC}, \lambda=220 \mathrm{~nm}$, hexanes $:{ }^{i} \mathrm{PrOH}=98: 2$, flow rate $\left.=1.0 \mathrm{~mL} / \mathrm{min}\right)$.

$\mathrm{mV}$

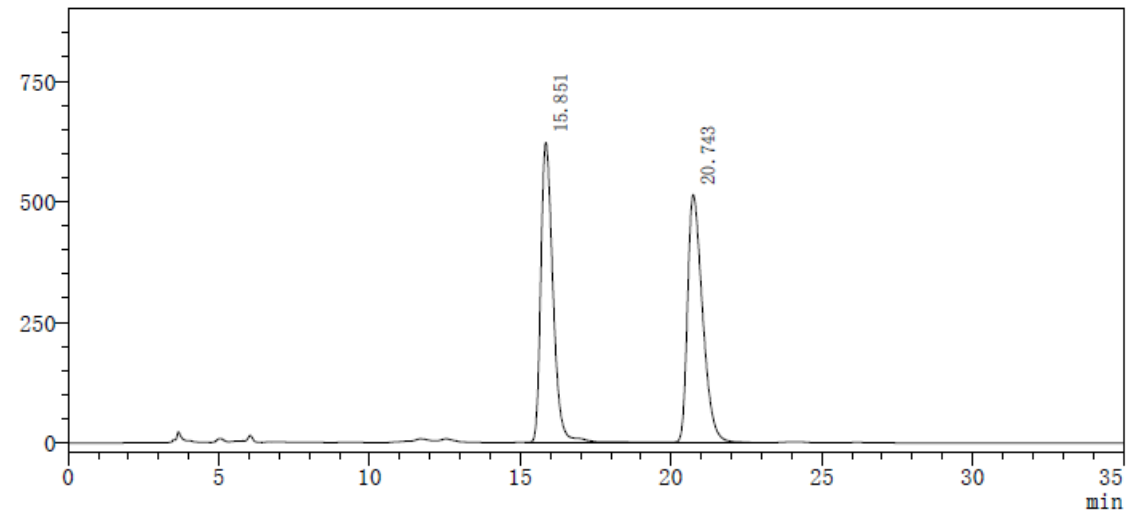

检测器A $220 \mathrm{~nm}$
\begin{tabular}{|c|c|c|c|c|c|}
\hline Peak\# & Time & Area & Height & Height\% & Area\% \\
\hline 1 & 15.851 & 17529041 & 622024 & 54.766 & 49.601 \\
\hline 2 & 20.743 & 17811051 & 513769 & 45.234 & 50.399 \\
\hline 总计 & & 35340092 & 1135793 & 100.000 & 100.000 \\
\hline
\end{tabular}


$\mathrm{mV}$

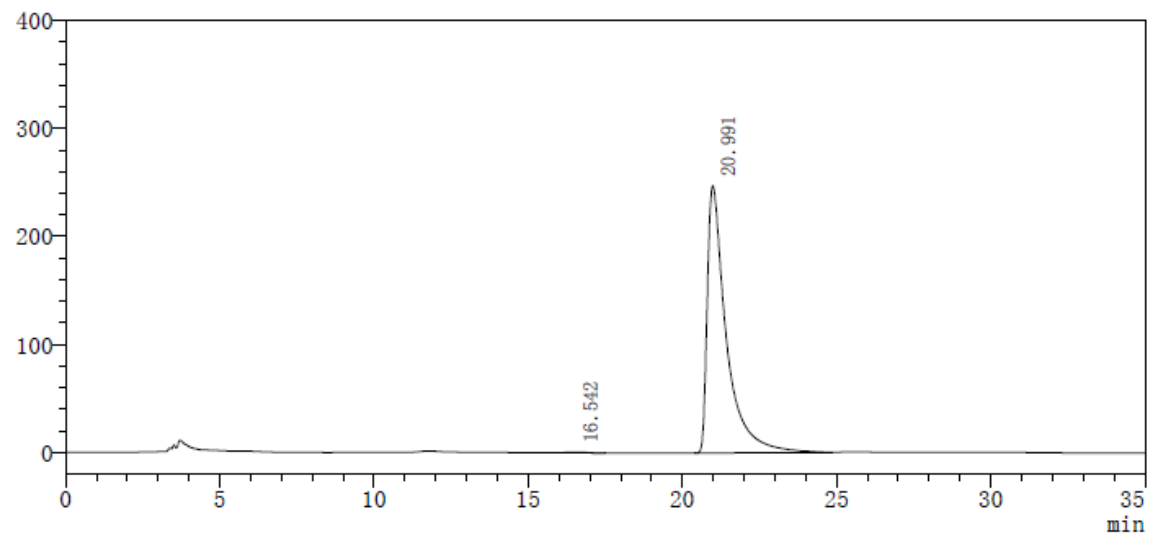

检测器A $220 \mathrm{~nm}$
\begin{tabular}{|c|c|c|c|c|c|}
\hline Peak\# & Time & Area & Height & Height\% & Area\% \\
\hline 1 & 16.542 & 27585 & 690 & 0.278 & 0.257 \\
\hline 2 & 20.991 & 10691749 & 247428 & 99.722 & 99.743 \\
\hline 总计 & & 10719334 & 248118 & 100.000 & 100.000 \\
\hline
\end{tabular}<smiles>CC(=O)C(C)(N)C(C)C=Cc1ccccc1</smiles>

$(2 S, 3 R)-3$ ao

isopropyl $(2 S, 3 R, E)$-2-amino-2,3-dimethyl-5-phenylpent-4-enoate

Prepared according to General Procedure B using Pd-6 and $(S, S p)$-L2-Cu, Purification by flash chromatography (PE/EA $=5: 1$ to $1: 1$ ) afforded the product as a pale yellow oil, $44.2 \mathrm{mg}, 85 \%$ yield, $>20: 1 \mathrm{dr},>99 \%$ ee, $\mathrm{R}_{\mathrm{f}}=0.5$ (PE/EA, 1:1).

$[\alpha]^{25} \mathrm{D}=66.9\left(\mathrm{c} 1.0, \mathrm{CHCl}_{3}\right)$

${ }^{1} \mathbf{H}$ NMR (400 MHz, $\left.\mathbf{C D C l}_{3}\right) \delta 7.40-7.34(\mathrm{~m}, 2 \mathrm{H}), 7.29(\mathrm{dd}, J=8.5,6.8 \mathrm{~Hz}, 2 \mathrm{H}), 7.24-7.18(\mathrm{~m}$, $1 \mathrm{H}), 6.45(\mathrm{~d}, J=15.8 \mathrm{~Hz}, 1 \mathrm{H}), 6.19(\mathrm{dd}, J=15.9,9.1 \mathrm{~Hz}, 1 \mathrm{H}), 5.04$ (hept, $J=6.2 \mathrm{~Hz}, 1 \mathrm{H}), 2.65$ (dq, $J$ $=9.3,6.9 \mathrm{~Hz}, 1 \mathrm{H}), 1.61(\mathrm{~s}, 2 \mathrm{H}), 1.29(\mathrm{~s}, 3 \mathrm{H}), 1.26(\mathrm{dd}, J=6.3,4.0 \mathrm{~Hz}, 6 \mathrm{H}), 1.06(\mathrm{~d}, J=6.9 \mathrm{~Hz}, 3 \mathrm{H})$. ${ }^{13}$ C NMR (101 MHz, $\left.\mathbf{C D C l}_{3}\right) \delta$ 176.79, 137.36, 131.70, 130.81, 128.51, 127.21, 126.21, 68.45, 60.32, 45.74, 24.87, 21.86, 21.80, 15.65 .

HRMS (ESI) calcd. for $\mathrm{C}_{16} \mathrm{H}_{24} \mathrm{NO}_{2}{ }^{+}(\mathrm{M}+\mathrm{H})^{+}:$262.1807, Found: 262.1804

the ee value $\mathrm{was}>99 \%, \mathrm{t}_{\mathrm{r}}($ minor $)=13.604 \mathrm{~min}, \mathrm{t}_{\mathrm{r}}($ major $)=17.724 \mathrm{~min}($ Chiralcel $\mathrm{IC}, \lambda=220 \mathrm{~nm}$, hexanes $:{ }^{i} \mathrm{PrOH}=98: 2$, flow rate $=1.0 \mathrm{~mL} / \mathrm{min}$ ). 
$\mathrm{mV}$

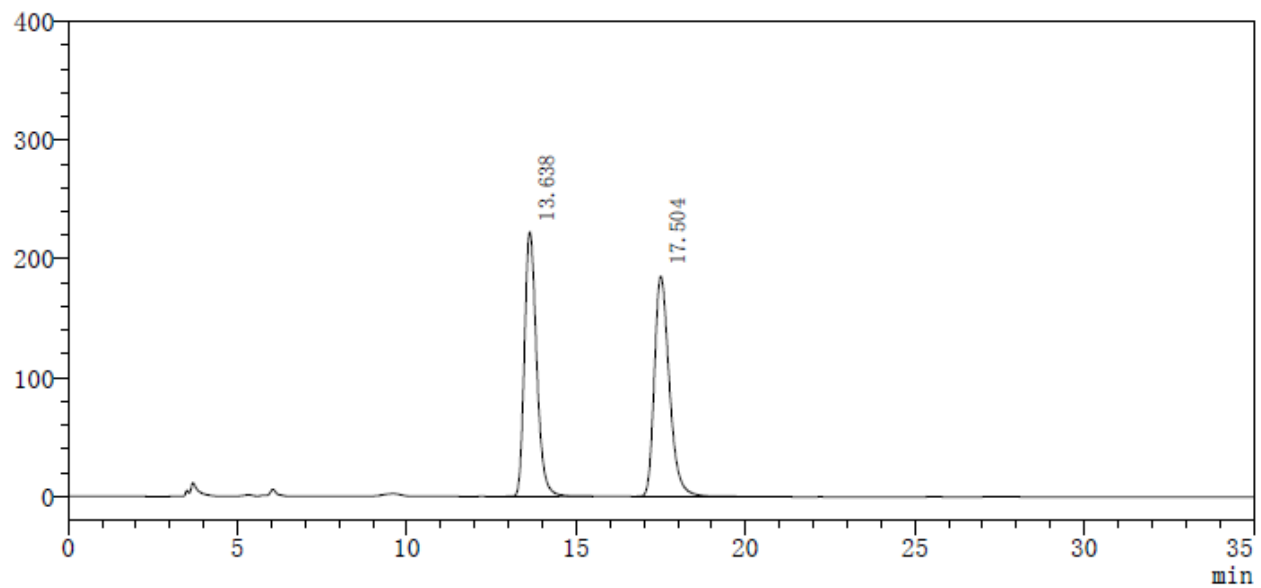

检测器A $220 \mathrm{~nm}$
\begin{tabular}{|c|c|c|c|c|c|}
\hline Peak\# & Time & Area & Height & Height\% & Area\% \\
\hline 1 & 13.638 & 5584079 & 222909 & 54.571 & 49.370 \\
\hline 2 & 17.504 & 5726606 & 185566 & 45.429 & 50.630 \\
\hline 总计 & & 11310685 & 408475 & 100.000 & 100.000 \\
\hline
\end{tabular}

$\mathrm{mV}$

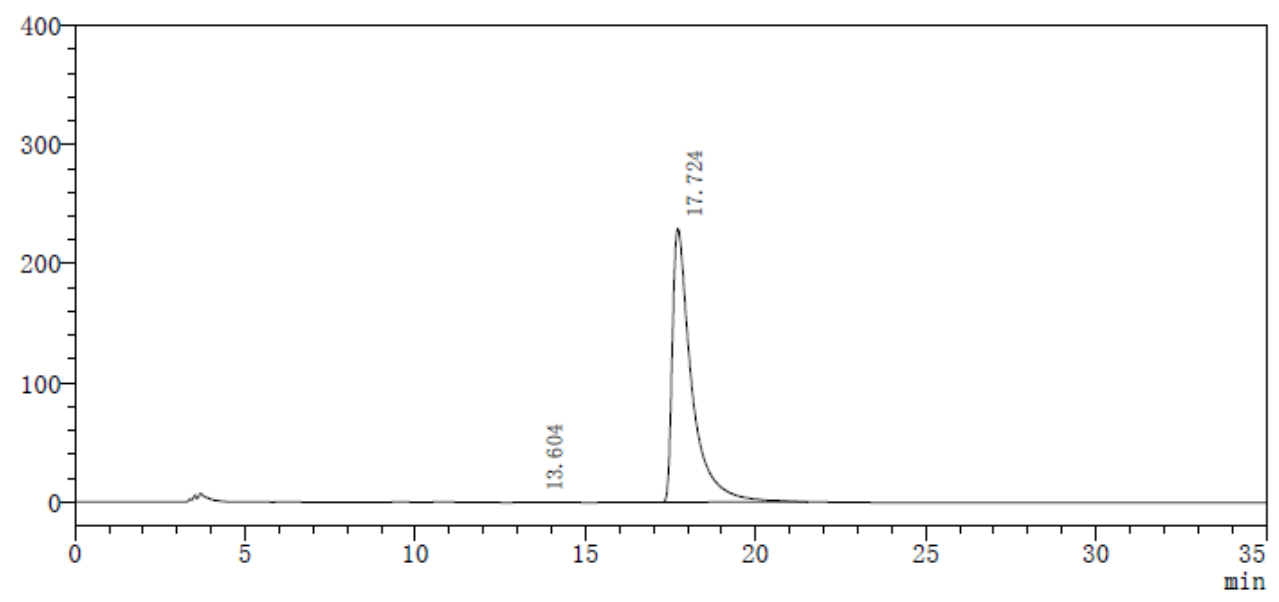

检测器A $220 \mathrm{~nm}$
\begin{tabular}{|c|c|c|c|c|c|}
\hline Peak\# & Time & Area & Height & Height\% & Area $\%$ \\
\hline 1 & 13.604 & 5779 & 137 & 0.059 & 0.064 \\
\hline 2 & 17.724 & 9032232 & 229664 & 99.941 & 99.936 \\
\hline 总计 & & 9038012 & 229801 & 100.000 & 100.000 \\
\hline
\end{tabular}

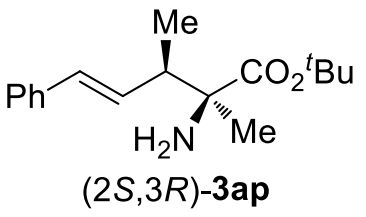

\section{tert-butyl $(2 S, 3 R, E)$-2-amino-2,3-dime thyl-5-phenylpent-4-enoate}

Prepared according to General Procedure B using Pd-6 and $(S, S p)$-L2-Cu, Purification by flash chromatography $(\mathrm{PE} / \mathrm{EA}=10: 1$ to $5: 1)$ afforded the product as a pale yellow solid, $52.5 \mathrm{mg}$, 95\% 
yield, m.p. $60-61{ }^{\circ} \mathrm{C},>20: 1 \mathrm{dr},>99 \%$ ee, $\mathrm{R}_{\mathrm{f}}=0.3$ (PE/EA, 5:1).

$[\alpha]^{25} \mathrm{D}=64.7\left(\mathrm{c} 1.0, \mathrm{CHCl}_{3}\right)$

${ }^{1}$ H NMR (400 MHz, CDCl $) \delta 7.40-7.36(\mathrm{~m}, 2 \mathrm{H}), 7.30(\mathrm{dd}, J=8.5,6.8 \mathrm{~Hz}, 2 \mathrm{H}), 7.24-7.16(\mathrm{~m}$, $1 \mathrm{H}), 6.45(\mathrm{~d}, J=15.9 \mathrm{~Hz}, 1 \mathrm{H}), 6.20(\mathrm{dd}, J=15.9,9.1 \mathrm{~Hz}, 1 \mathrm{H}), 2.62(\mathrm{dq}, J=9.2,6.9 \mathrm{~Hz}, 1 \mathrm{H}), 1.63-$ $1.57(\mathrm{~m}, 2 \mathrm{H}), 1.48(\mathrm{~s}, 9 \mathrm{H}), 1.27$ (s, 3H), 1.07 (d, $J=6.9 \mathrm{~Hz}, 3 \mathrm{H})$.

${ }^{13}$ C NMR (101 MHz, $\left.\mathbf{C D C l}_{3}\right) \delta$ 176.52, 137.41, 131.56, 131.04, 128.51, 127.17, 126.19, 81.00, 60.58, 45.73, 28.02, 25.04, 15.66.

HRMS (ESI) calcd. for $\mathrm{C}_{17} \mathrm{H}_{25} \mathrm{NO}_{2}{ }^{+}(\mathrm{M}+\mathrm{H})^{+}:$276.1964, Found: 276.1954

the ee value was $>99 \%, \mathrm{t}_{\mathrm{r}}($ minor $)=10.363 \mathrm{~min}, \mathrm{t}_{\mathrm{r}}($ major $)=12.258 \mathrm{~min}($ Chiralcel $\mathrm{IC}, \lambda=220 \mathrm{~nm}$, hexanes $:{ }^{i} \mathrm{PrOH}=98: 2$, flow rate $=1.0 \mathrm{~mL} / \mathrm{min}$ ).

$\mathrm{mV}$

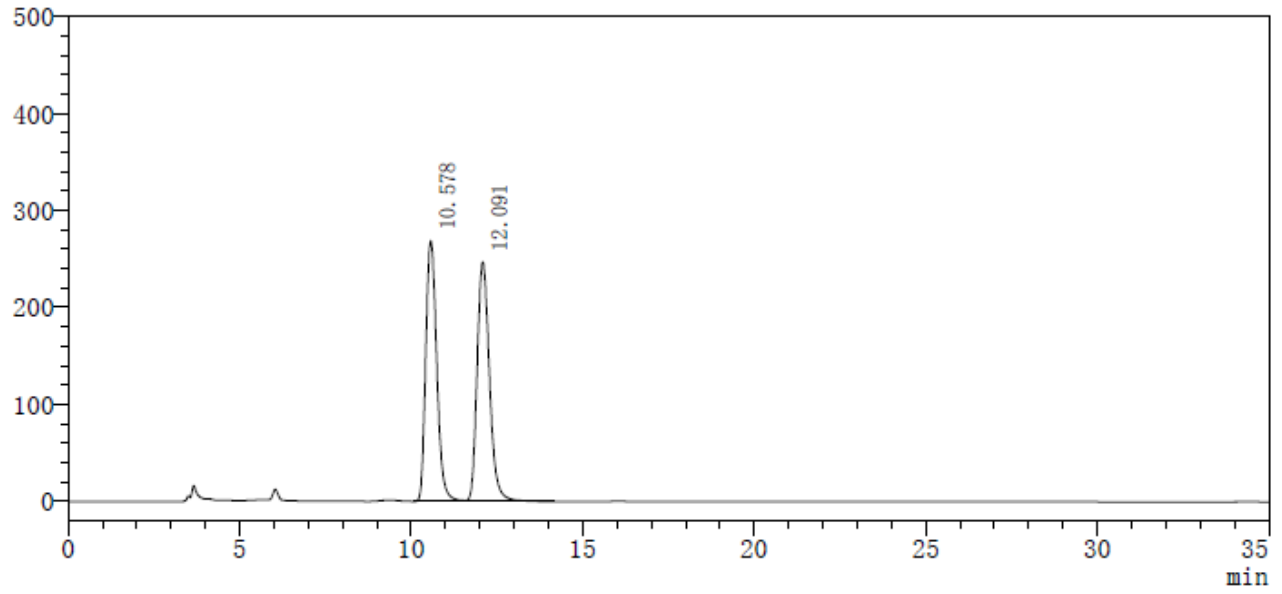

检测器A 220nm
\begin{tabular}{|c|c|c|c|c|c|}
\hline Peak\# & Time & Area & Height & Height\% & Area\% \\
\hline 1 & 10.578 & 5987949 & 268430 & 52.121 & 49.271 \\
\hline 2 & 12.091 & 6165146 & 246584 & 47.879 & 50.729 \\
\hline 总计 & & 12153096 & 515014 & 100.000 & 100.000 \\
\hline
\end{tabular}




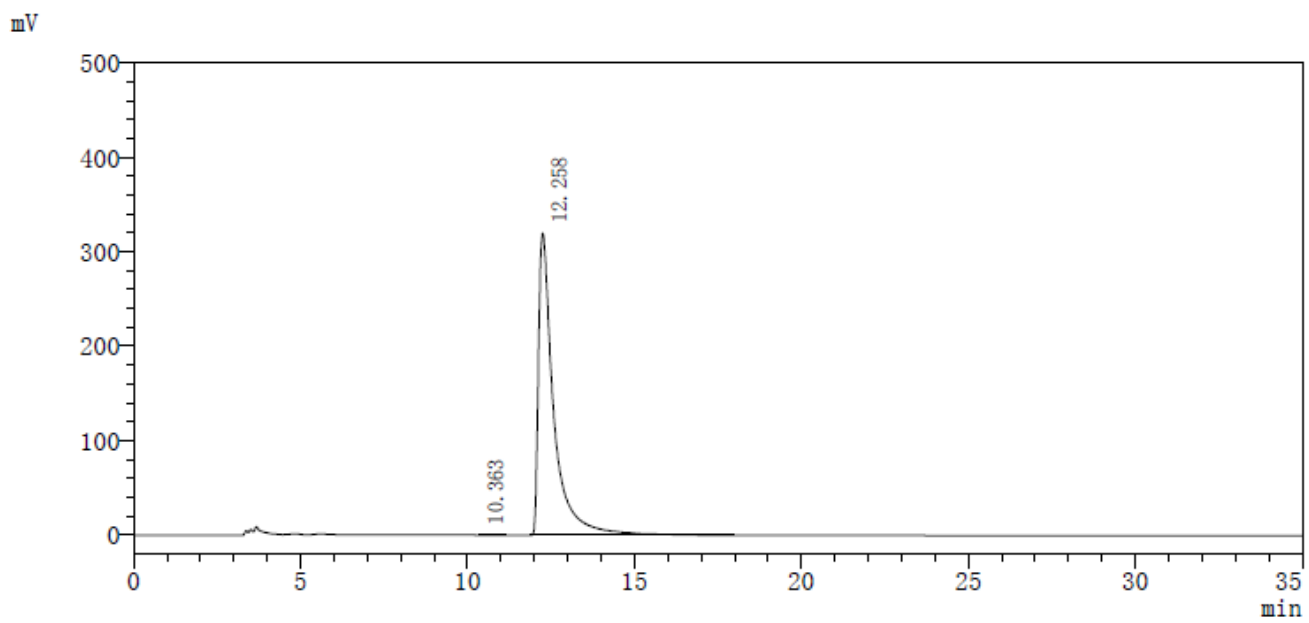

检测器A 220nm
\begin{tabular}{|c|c|c|c|c|c|}
\hline Peak\# & Time & Area & Height & Height\% & Area\% \\
\hline 1 & 10.363 & 3957 & 193 & 0.060 & 0.039 \\
\hline 2 & 12.258 & 10246498 & 319492 & 99.940 & 99.961 \\
\hline 总计 & & 10250456 & 319685 & 100.000 & 100.000 \\
\hline
\end{tabular}<smiles>CC(=O)C(N=C(c1ccccc1)c1ccccc1)C(C)/C=C/c1ccccc1</smiles>

methyl (2S,3R,E)-2-((diphenylmethylene)amino)-3-methyl-5-phenylpent-4-enoate

Prepared according to General Procedure B using Pd-6 and $(S, S p)-\mathbf{L 2}-\mathbf{C u}$, Purification by flash chromatography $(\mathrm{PE} / \mathrm{EA}=50: 1$ to 10:1) afforded the product as a pale yellow oil, $65 \mathrm{mg}, 85 \%$ yield, $>20: 1 \mathrm{dr},>99 \%$ ee, $\mathrm{R}_{\mathrm{f}}=0.4(\mathrm{PE} / \mathrm{EA}, 10: 1)$.

$[\alpha]^{25} \mathrm{D}=-78.1\left(\mathrm{c} 1.0, \mathrm{CHCl}_{3}\right)$

${ }^{1}$ H NMR (400 MHz, $\left.\mathbf{C D C l}_{3}\right) \delta 7.70-7.64(\mathrm{~m}, 2 \mathrm{H}), 7.45-7.37(\mathrm{~m}, 4 \mathrm{H}), 7.36-7.31(\mathrm{~m}, 2 \mathrm{H}), 7.30$ $7.22(\mathrm{~m}, 4 \mathrm{H}), 7.18(\mathrm{ddd}, J=8.5,5.3,2.5 \mathrm{~Hz}, 1 \mathrm{H}), 7.13-7.08(\mathrm{~m}, 2 \mathrm{H}), 6.39(\mathrm{dd}, J=15.9,1.1 \mathrm{~Hz}, 1 \mathrm{H})$, $5.99(\mathrm{dd}, J=15.9,8.2 \mathrm{~Hz}, 1 \mathrm{H}), 4.08(\mathrm{~d}, J=6.6 \mathrm{~Hz}, 1 \mathrm{H}), 3.68(\mathrm{~s}, 3 \mathrm{H}), 3.13(\mathrm{dtd}, J=7.9,6.6,1.1 \mathrm{~Hz}$, $1 \mathrm{H}), 1.18(\mathrm{~d}, J=6.8 \mathrm{~Hz}, 3 \mathrm{H})$.

${ }^{13}$ C NMR (101 MHz, CDCl 3$) \delta 172.05,171.15,139.66,137.43,136.45,132.12,130.46,130.36$, $128.98,128.71,128.52,128.50,128.13,128.03,127.18,126.18,70.63,52.00,41.57,16.41$. HRMS (ESI) calcd. for $\mathrm{C}_{26} \mathrm{H}_{26} \mathrm{NO}_{2}{ }^{+}(\mathrm{M}+\mathrm{H})^{+}: 384.1964$, Found: 384.1960

the ee value $\mathrm{was}>99 \%, \mathrm{t}_{\mathrm{r}}($ minor $)=15.386 \mathrm{~min}, \mathrm{t}_{\mathrm{r}}$ (major) $=21.322 \mathrm{~min}$ (Chiralcel $\mathrm{IC}, \lambda=220 \mathrm{~nm}$, hexanes $:{ }^{i} \mathrm{PrOH}=98: 2$, flow rate $=1.0 \mathrm{~mL} / \mathrm{min}$ ). 
$\mathrm{mV}$

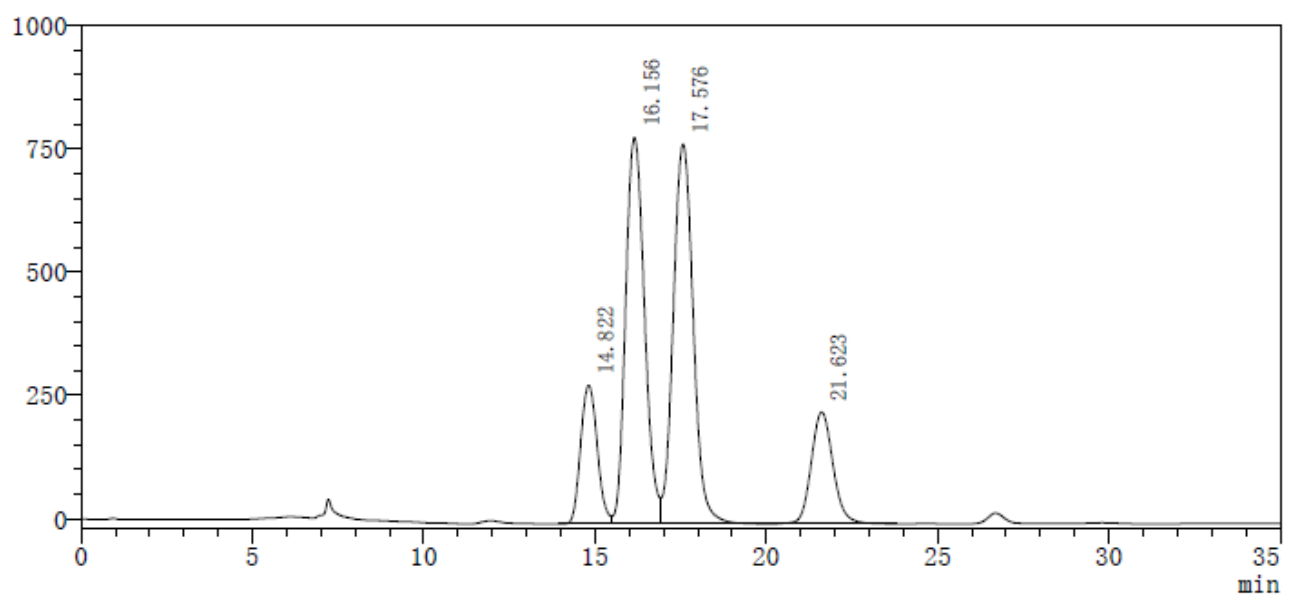

检测器 $\mathrm{A} 220 \mathrm{~nm}$

\begin{tabular}{|c|c|c|c|c|c|}
\hline Peak\# & Time & Area & Height & Height\% & Area\% \\
\hline 1 & 14.822 & 9508740 & 280980 & 13.635 & 11.780 \\
\hline 2 & 16.156 & 29995011 & 783266 & 38.009 & 37.161 \\
\hline 3 & 17.576 & 31177409 & 769847 & 37.358 & 38.626 \\
\hline 4 & 21.623 & 10035817 & 226652 & 10.999 & 12.433 \\
\hline 总计 & & 80716977 & 2060744 & 100.000 & 100.000 \\
\hline
\end{tabular}

$\mathrm{mV}$

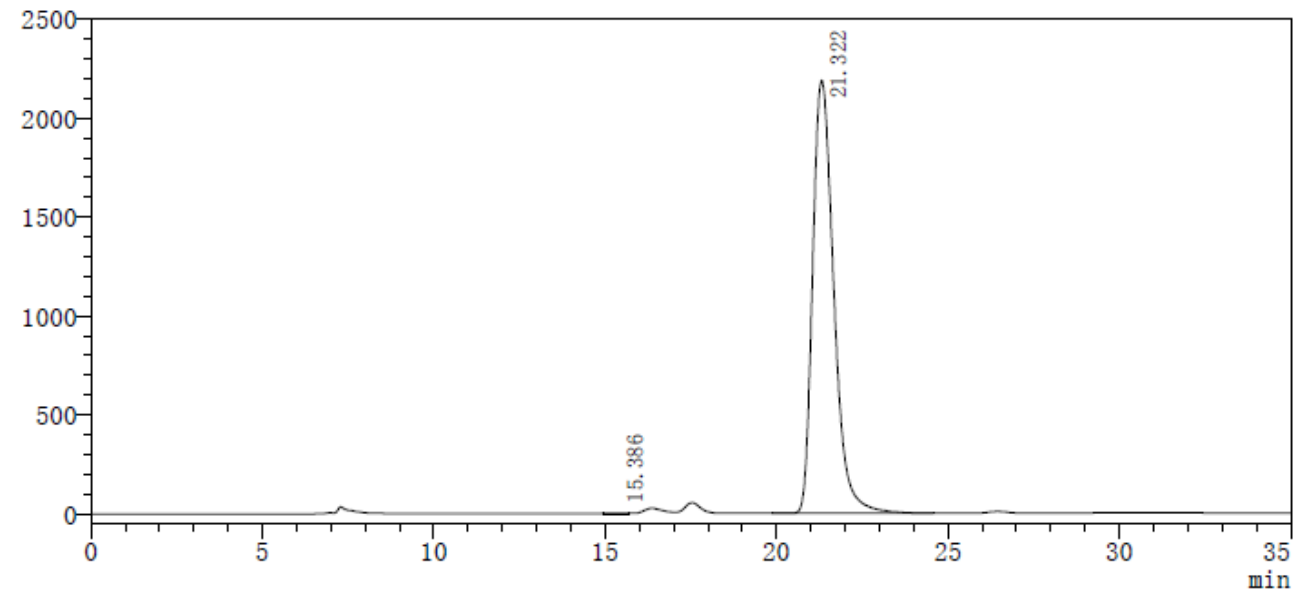

检测器A $220 \mathrm{~nm}$
\begin{tabular}{|c|c|c|c|c|c|}
\hline Peak\# & Time & Area & Height & Height\% & Area\% \\
\hline 1 & 15.386 & 55148 & 1420 & 0.065 & 0.059 \\
\hline 2 & 21.322 & 93681605 & 2187477 & 99.935 & 99.941 \\
\hline 总计 & & 93736753 & 2188898 & 100.000 & 100.000 \\
\hline
\end{tabular}<smiles>CC(=O)C(C(=NC(=[18O])c1ccccc1)c1ccccc1)C(C)C=Cc1ccccc1</smiles>

methyl $(2 R, 3 R, E)-2-(($ diphenylmethylene)amino)-3-methyl-5-phenylpent-4-enoate 
Prepared according to General Procedure B using Pd-6 and $(R, R p)-\mathbf{L 2}-\mathbf{C u}$, Purification by flash chromatography (PE/EA $=50: 1$ to 10:1) afforded the product as a pale yellow solid, $65.3 \mathrm{mg}, 85 \%$ yield, m.p. $82-83{ }^{\circ} \mathrm{C},>20: 1 \mathrm{dr},>99 \%$ ee, $\mathrm{R}_{\mathrm{f}}=0.4$ (PE/EA, 10:1).

$[\alpha]^{25} \mathrm{D}=68.0\left(\mathrm{c} 1.0, \mathrm{CHCl}_{3}\right)$

${ }^{1} \mathbf{H}$ NMR (400 MHz, $\left.\mathbf{C D C l}_{3}\right) \delta 7.68-7.61(\mathrm{~m}, 2 \mathrm{H}), 7.45-7.41(\mathrm{~m}, 3 \mathrm{H}), 7.39-7.36(\mathrm{~m}, 1 \mathrm{H}), 7.36-$ $7.25(\mathrm{~m}, 6 \mathrm{H}), 7.22-7.16(\mathrm{~m}, 1 \mathrm{H}), 7.12-7.07(\mathrm{~m}, 2 \mathrm{H}), 6.43(\mathrm{~d}, J=15.9 \mathrm{~Hz}, 1 \mathrm{H}), 6.20(\mathrm{dd}, J=15.9$, $8.5 \mathrm{~Hz}, 1 \mathrm{H}), 4.08(\mathrm{~d}, J=5.9 \mathrm{~Hz}, 1 \mathrm{H}), 3.71(\mathrm{~s}, 3 \mathrm{H}), 3.14-3.01(\mathrm{~m}, 1 \mathrm{H}), 1.06(\mathrm{~d}, J=6.9 \mathrm{~Hz}, 3 \mathrm{H})$.

${ }^{13}$ C NMR (101 MHz, CDCl 3$) \delta 171.04,169.89,138.63,136.50,135.45,130.96,129.58,129.29$, $127.86,127.61,127.45,127.39,126.99,126.91,126.00,125.16,69.84,50.95,40.62,16.57$. HRMS (ESI) calcd. for $\mathrm{C}_{26} \mathrm{H}_{26} \mathrm{NO}_{2}{ }^{+}(\mathrm{M}+\mathrm{H})^{+}: 384.1964$, Found: 384.1960

the ee value $\mathrm{was}>99 \%, \mathrm{t}_{\mathrm{r}}($ minor $)=16.160 \mathrm{~min}, \mathrm{t}_{\mathrm{r}}($ major $)=17.588 \mathrm{~min}$ (Chiralcel IC, $\lambda=220 \mathrm{~nm}$, hexanes $:{ }^{i} \mathrm{PrOH}=98: 2$, flow rate $=1.0 \mathrm{~mL} / \mathrm{min}$ ).

$\mathrm{mV}$

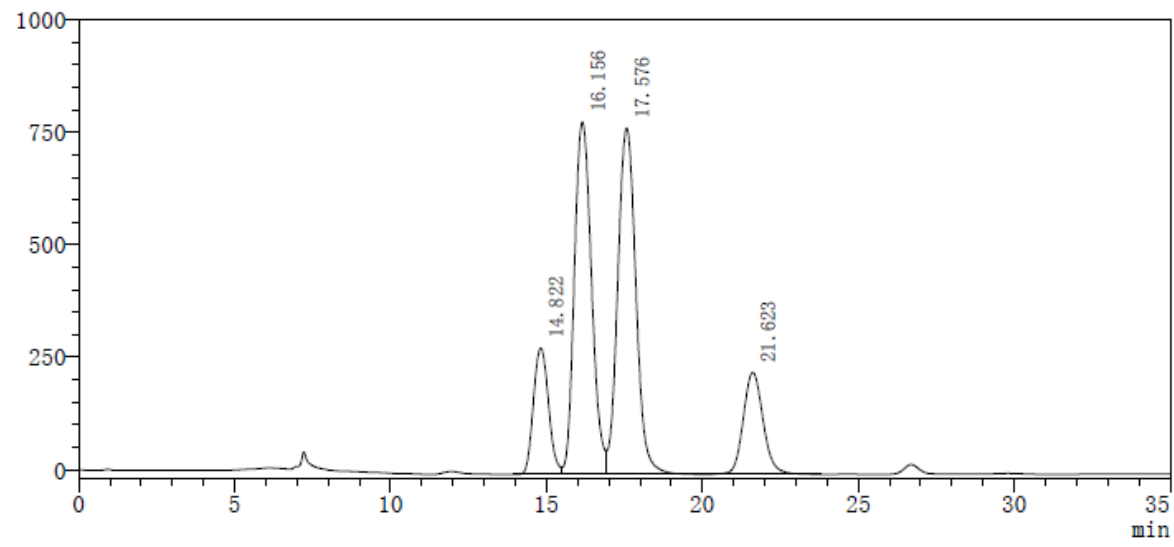

检测器A 220nm
\begin{tabular}{|c|c|c|c|c|c|}
\hline Peak\# & Time & Area & Height & Height\% & Area\% \\
\hline 1 & 14.822 & 9508740 & 280980 & 13.635 & 11.780 \\
\hline 2 & 16.156 & 29995011 & 783266 & 38.009 & 37.161 \\
\hline 3 & 17.576 & 31177409 & 769847 & 37.358 & 38.626 \\
\hline 4 & 21.623 & 10035817 & 226652 & 10.999 & 12.433 \\
\hline 总计 & & 80716977 & 2060744 & 100.000 & 100.000 \\
\hline
\end{tabular}


$\mathrm{mV}$

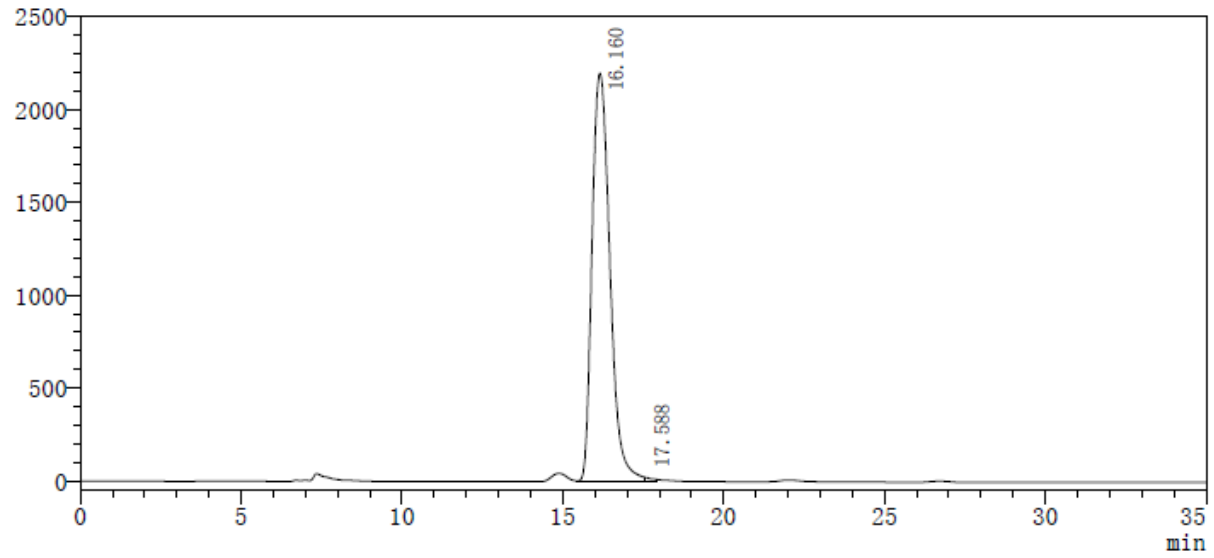

检测器A $220 \mathrm{~nm}$
\begin{tabular}{|c|c|c|c|c|c|}
\hline Peak\# & Time & Area & Height & Height $\%$ & Area\% \\
\hline 1 & 16.160 & 85106580 & 2198910 & 98.990 & 99.587 \\
\hline 2 & 17.588 & 352768 & 22427 & 1.010 & 0.413 \\
\hline 总计 & & 85459348 & 2221337 & 100.000 & 100.000 \\
\hline
\end{tabular}

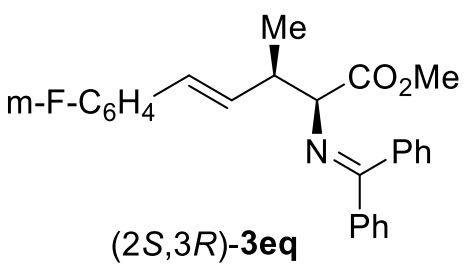

methyl (2S,3R,E)-2-((diphenylmethylene)amino)-5-(3-fluorophenyl)-3-methylpent-4-enoate

Prepared according to General Procedure B using Pd-6 and $(S, S p)$-L2-Cu, Purification by flash chromatography ( $\mathrm{PE} / \mathrm{EA}=50: 1$ to $10: 1)$ afforded the product as a pale yellow solid, $57 \mathrm{mg}, 92 \%$ yield, m.p. $79-81{ }^{\circ} \mathrm{C},>20: 1 \mathrm{dr},>99 \%$ ee, $\mathrm{R}_{\mathrm{f}}=0.3$ (PE/EA, 10:1).

$[\alpha]^{25} \mathrm{D}=-76.5\left(\mathrm{c} 1.0, \mathrm{CHCl}_{3}\right)$

${ }^{1}$ H NMR (400 MHz, CDCl 3$) \delta 7.70-7.63(\mathrm{~m}, 2 \mathrm{H}), 7.47-7.37(\mathrm{~m}, 4 \mathrm{H}), 7.37-7.30(\mathrm{~m}, 2 \mathrm{H}), 7.21$ $(\mathrm{td}, \mathrm{J}=8.0,6.0 \mathrm{~Hz}, 1 \mathrm{H}), 7.12-7.08(\mathrm{~m}, 2 \mathrm{H}), 7.03(\mathrm{dt}, \mathrm{J}=7.7,1.3 \mathrm{~Hz}, 1 \mathrm{H}), 6.97(\mathrm{dt}, \mathrm{J}=10.3,2.1 \mathrm{~Hz}$, $1 \mathrm{H}), 6.87$ (tdd, J = 8.4, 2.6, $1.0 \mathrm{~Hz}, 1 \mathrm{H}), 6.35(\mathrm{~d}, \mathrm{~J}=15.9 \mathrm{~Hz}, 1 \mathrm{H}), 6.00(\mathrm{dd}, \mathrm{J}=15.9,8.1 \mathrm{~Hz}, 1 \mathrm{H})$, $4.08(\mathrm{~d}, \mathrm{~J}=6.5 \mathrm{~Hz}, 1 \mathrm{H}), 3.69(\mathrm{~s}, 3 \mathrm{H}), 3.12(\mathrm{dtd}, \mathrm{J}=7.9,6.7,1.1 \mathrm{~Hz}, 1 \mathrm{H}), 1.18(\mathrm{~d}, \mathrm{~J}=6.8 \mathrm{~Hz}, 3 \mathrm{H})$.

${ }^{13}$ C NMR (101 MHz, CDCl $) \delta 171.93,171.24,163.10(\mathrm{~d}, J=245.0 \mathrm{~Hz}), 139.81(\mathrm{~d}, J=7.6 \mathrm{~Hz})$, 139.58, 136.38, 133.63, 130.50, 129.90 (d, $J=8.4 \mathrm{~Hz}), 129.31$ (d, $J=2.6 \mathrm{~Hz}), 128.96,128.76,128.54$, 128.14, 127.99, 122.08 (d, $J=2.7 \mathrm{~Hz}), 113.94$ (d, $J=21.5 \mathrm{~Hz}), 112.54$ (d, $J=21.7 \mathrm{~Hz}), 70.41,52.01$, $41.45,16.25$.

${ }^{19}$ F NMR (376 MHz, $\left.\mathbf{C D C l}_{3}\right) \delta-118.75$

HRMS (ESI) calcd. for $\mathrm{C}_{16} \mathrm{H}_{24} \mathrm{FNO}_{2}{ }^{+}(\mathrm{M}+\mathrm{H})^{+}:$: 402.1864, Found: 402.1865

the ee value $\mathrm{w}$ as $>99 \%, \mathrm{t}_{\mathrm{r}}($ minor $)=14.288 \mathrm{~min}, \mathrm{t}_{\mathrm{r}}($ major $)=20.877 \mathrm{~min}$ (Chiralcel $\mathrm{IC}, \lambda=220 \mathrm{~nm}$, hexanes : ${ }^{i} \mathrm{PrOH}=98: 2$, flow rate $=1.0 \mathrm{~mL} / \mathrm{min}$ ). 
$\mathrm{mV}$

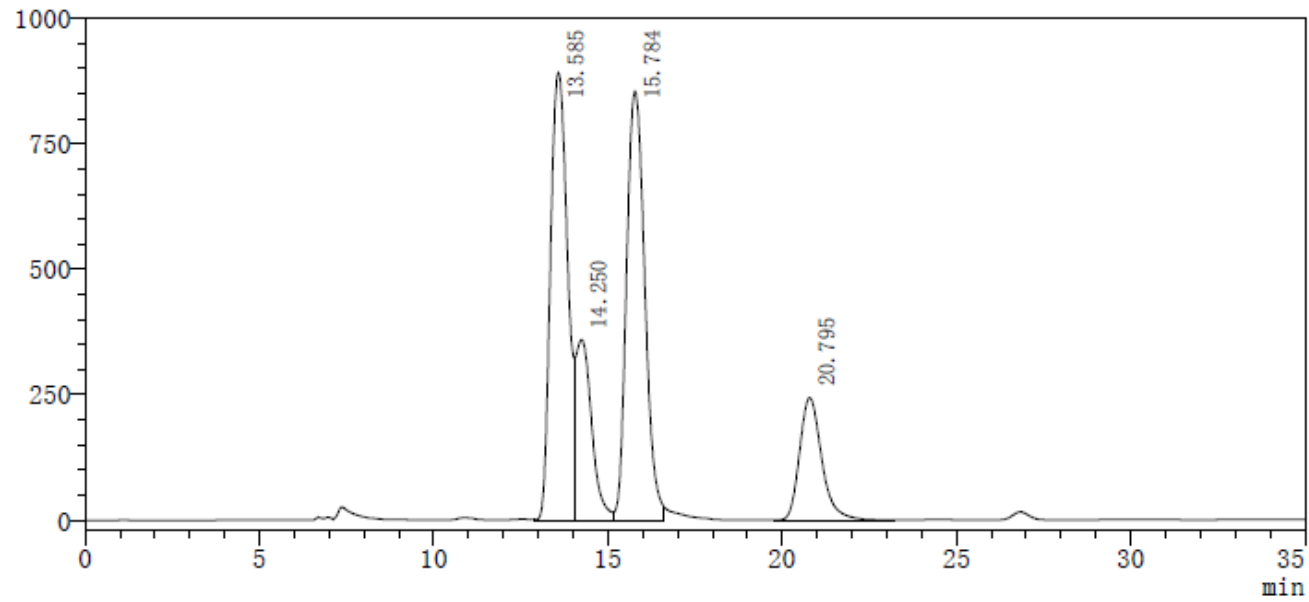

检测器A $220 \mathrm{~nm}$
\begin{tabular}{|c|c|c|c|c|c|}
\hline Peak\# & Time & Area & Height & Height\% & Area\% \\
\hline 1 & 13.585 & 30531771 & 891210 & 37.941 & 36.369 \\
\hline 2 & 14.250 & 11449447 & 359350 & 15.298 & 13.638 \\
\hline 3 & 15.784 & 31203046 & 854347 & 36.372 & 37.169 \\
\hline 4 & 20.795 & 10765643 & 244023 & 10.389 & 12.824 \\
\hline 总计 & 83949907 & 2348930 & 100.000 & 100.000 \\
\hline
\end{tabular}

$\mathrm{mV}$

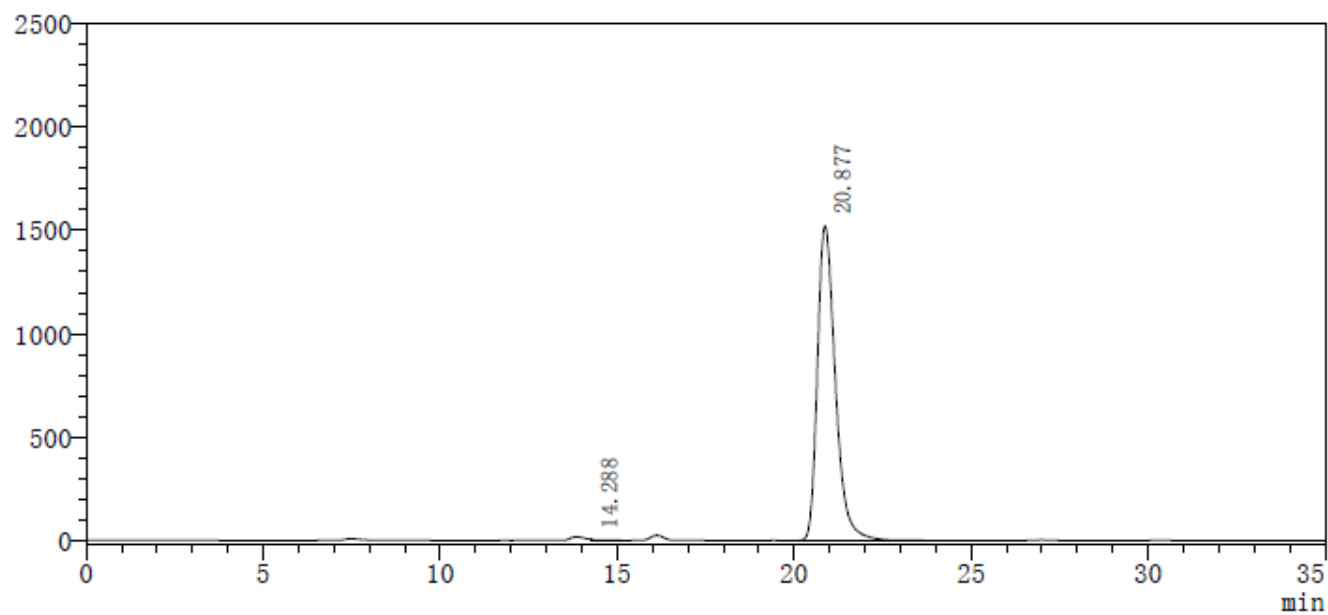

检测器A $220 \mathrm{~nm}$
\begin{tabular}{|c|c|c|c|c|c|}
\hline Peak\# & Time & Area & Height & Height\% & Area\% \\
\hline 1 & 14.288 & 74912 & 4697 & 0.307 & 0.139 \\
\hline 2 & 20.877 & 53755071 & 1522762 & 99.693 & 99.861 \\
\hline 总计 & & 53829983 & 1527459 & 100.000 & 100.000 \\
\hline
\end{tabular}

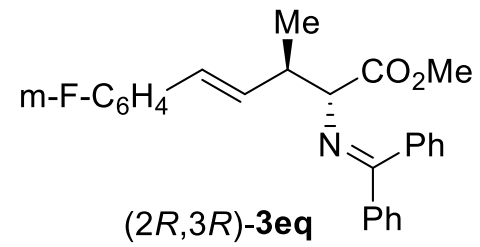

methyl (2R,3R,E)-2-((diphenylmethylene)amino)-5-(3-fluorophenyl)-3-methylpent-4-enoate 
Prepared according to General Procedure B using Pd-6 and $(R, R p)-\mathbf{L 2}-\mathbf{C u}$, Purification by flash chromatography $(\mathrm{PE} / \mathrm{EA}=50: 1$ to 10:1) afforded the product as a pale yellow oil, $58.3 \mathrm{mg}, 94 \%$ yield, $>20: 1 \mathrm{dr},>99 \%$ ee, $\mathrm{R}_{\mathrm{f}}=0.3$ (PE/EA, 10:1).

$[\alpha]^{25} \mathrm{D}=62.1\left(\mathrm{c} 1.0, \mathrm{CHCl}_{3}\right)$

${ }^{1}$ H NMR (400 MHz, $\left.\mathbf{C D C l}_{3}\right) \delta 7.64(\mathrm{dt}, J=7.1,1.6 \mathrm{~Hz}, 2 \mathrm{H}), 7.47-7.41(\mathrm{~m}, 3 \mathrm{H}), 7.38(\mathrm{dd}, J=6.8$, $1.7 \mathrm{~Hz}, 1 \mathrm{H}), 7.32(\mathrm{ddd}, J=8.5,6.4,1.6 \mathrm{~Hz}, 2 \mathrm{H}), 7.27-7.19(\mathrm{~m}, 1 \mathrm{H}), 7.12-7.07$ (m, 3H), $7.03(\mathrm{dd}, J$ $=10.4,2.0 \mathrm{~Hz}, 1 \mathrm{H}), 6.88(\mathrm{tdd}, J=8.4,2.6,1.1 \mathrm{~Hz}, 1 \mathrm{H}), 6.40(\mathrm{~d}, J=15.9 \mathrm{~Hz}, 1 \mathrm{H}), 6.24(\mathrm{ddd}, J=16.0$, $8.4,1.7 \mathrm{~Hz}, 1 \mathrm{H}), 4.08(\mathrm{dd}, J=5.8,1.7 \mathrm{~Hz}, 1 \mathrm{H}), 3.71(\mathrm{~d}, J=1.7 \mathrm{~Hz}, 3 \mathrm{H}), 3.12-3.00(\mathrm{~m}, 1 \mathrm{H}), 1.05(\mathrm{dd}$, $J=6.9,1.8 \mathrm{~Hz}, 3 \mathrm{H})$.

${ }^{13}$ C NMR (101 MHz, $\left.\mathbf{C D C l}_{3}\right) \delta 172.01,171.15,163.12(\mathrm{~d}, J=244.6 \mathrm{~Hz}), 139.96(\mathrm{~d}, J=7.5 \mathrm{~Hz})$, 139.63, 136.46, 133.53, 130.46, 129.89 (d, $J=8.6 \mathrm{~Hz}), 129.65$ (d, $J=2.6 \mathrm{~Hz}), 128.93,128.77,128.58$, 128.11, 127.96, $122.16(\mathrm{~d}, J=2.9 \mathrm{~Hz}), 113.87(\mathrm{~d}, J=21.5 \mathrm{~Hz}), 112.62(\mathrm{~d}, J=21.7 \mathrm{~Hz}), 70.74,52.08$, 41.60, 17.59.

${ }^{19}$ F NMR (376 MHz, $\left.\mathrm{CDCl}_{3}\right) \delta-118.84$

HRMS (ESI) calcd. for $\mathrm{C}_{16} \mathrm{H}_{24} \mathrm{FNO}_{2}{ }^{+}(\mathrm{M}+\mathrm{H})^{+}:$402.1864, Found: 402.1863

the ee value $\mathrm{was}>99 \%, \mathrm{t}_{\mathrm{r}}($ minor $)=13.558 \mathrm{~min}, \mathrm{t}_{\mathrm{r}}$ (major) $=16.243 \mathrm{~min}$ (Chiralcel $\mathrm{IC}, \lambda=220 \mathrm{~nm}$, hexanes $:{ }^{i} \mathrm{PrOH}=98: 2$, flow rate $=1.0 \mathrm{~mL} / \mathrm{min}$ ). 
$\mathrm{mV}$

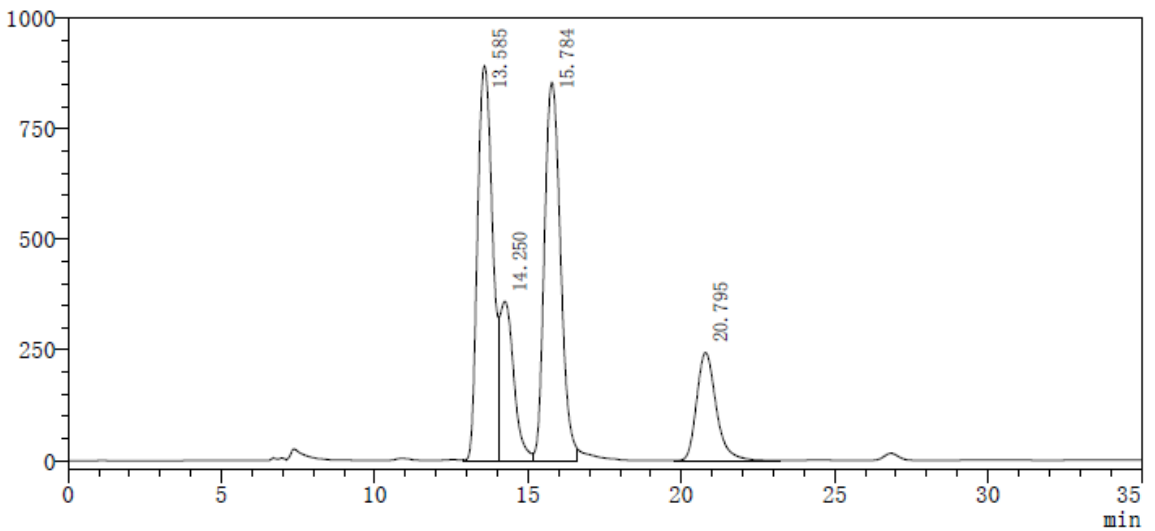

检测器A $220 \mathrm{~nm}$
\begin{tabular}{|c|c|c|c|c|c|}
\hline Peak\# & Time & Area & Height & Height\% & Area\% \\
\hline 1 & 13.585 & 30531771 & 891210 & 37.941 & 36.369 \\
\hline 2 & 14.250 & 11449447 & 359350 & 15.298 & 13.638 \\
\hline 3 & 15.784 & 31203046 & 854347 & 36.372 & 37.169 \\
\hline 4 & 20.795 & 10765643 & 244023 & 10.389 & 12.824 \\
\hline 总计 & & 83949907 & 2348930 & 100.000 & 100.000 \\
\hline
\end{tabular}

$\mathrm{mV}$

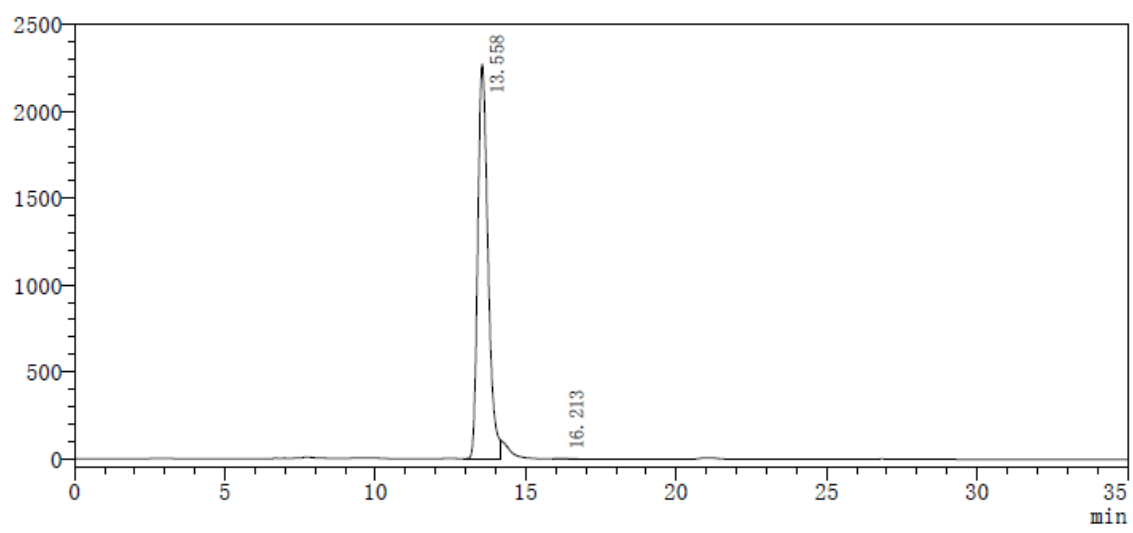

检测器A $220 \mathrm{~nm}$
\begin{tabular}{|c|c|c|c|c|c|}
\hline Peak\# & Time & Area & Height & Height\% & Area\% \\
\hline 1 & 13.558 & 53678498 & 2268910 & 99.988 & 99.986 \\
\hline 2 & 16.213 & 7646 & 281 & 0.012 & 0.014 \\
\hline 总计 & & 53686144 & 2269191 & 100.000 & 100.000 \\
\hline
\end{tabular}

\section{References}

1. a) Preuß, T.; Saak, W.; Doye, S. Chem. - Eur. J. 2013, 19, 3833-3837. b) Adamson, N. J.; Hull, E.; Malcolmson, S. J. J. Am. Chem. Soc. 2017, 139, 7180-7183.

2. a) Huo, X.; He, R.; Fu, J.; Zhang, J.; Yang, G.; Zhang, W. J. Am. Chem. Soc. 2017, 139, 9819-9822. b) Liu, P.; Huo, X.; Li, B.; He, R.; Zhang, J.; Xie, F.; Zhang, W. Org. Lett. 2018, 20, 6564-6568. c) Guerrero-Corella, A.; Esterban, F.; Iniesta, M.; Martín-Somer, A.; Parra, M.; Díaz-Tendero, S.; Fraile, A.; Alemán, J. Angew. Chem., Int. Ed. 2018, 57, 5350- 5354.

3. Adamson, N. J.; Wilbur, K. C. E.; Malcolmson, S. J. J. Am. Chem. Soc. 2018, 140, 2761-2764. 


\section{Copies of NMR Spectra}<smiles>COC(=O)C(C)(N)C(C)C=Cc1ccccc1</smiles>

$(2 S, 3 R)-3 \mathbf{a a}$

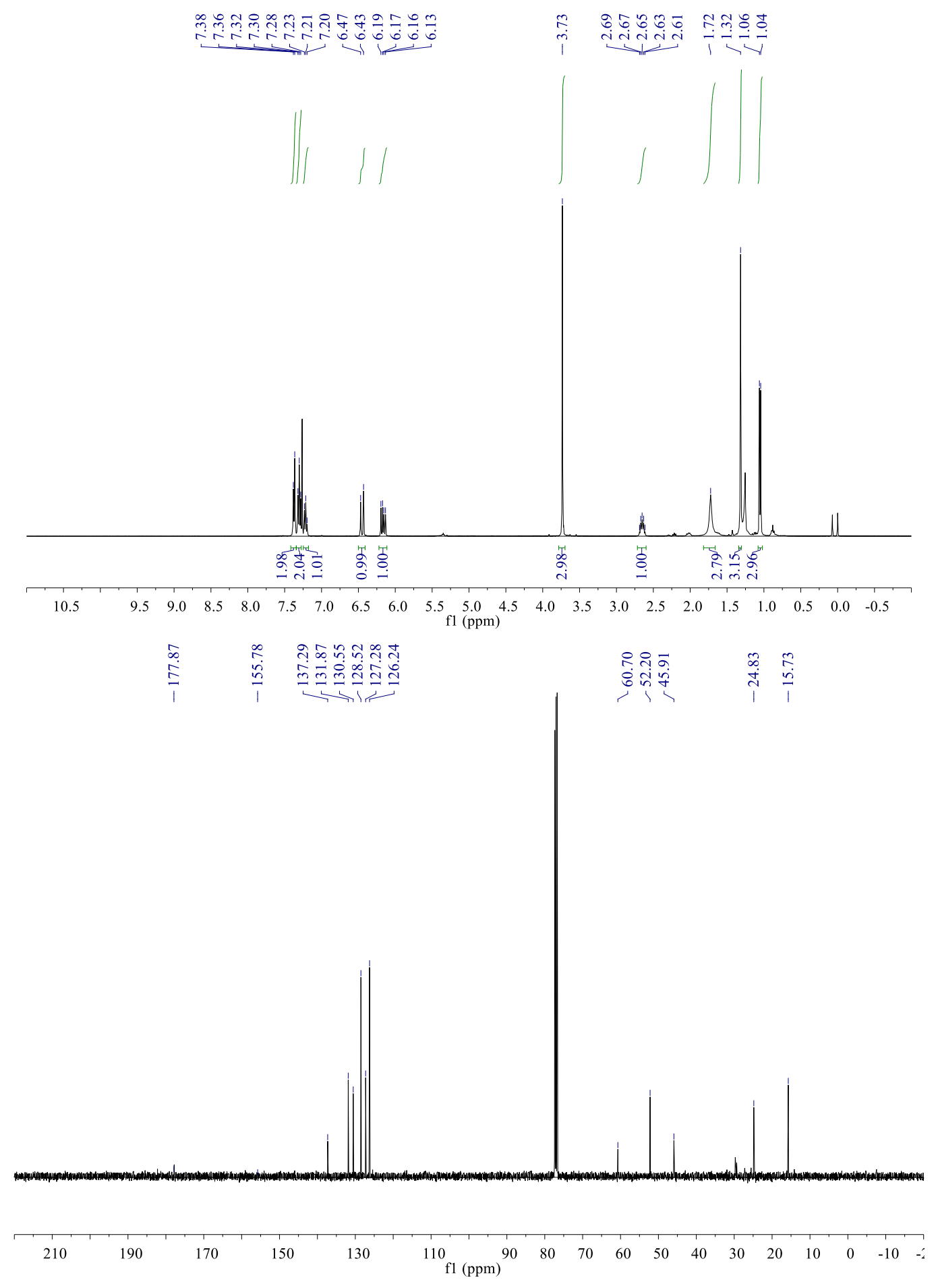


<smiles>COC(=O)C(C)(N)C(C)C=Cc1ccccc1</smiles>

(2R,3R)-3aa
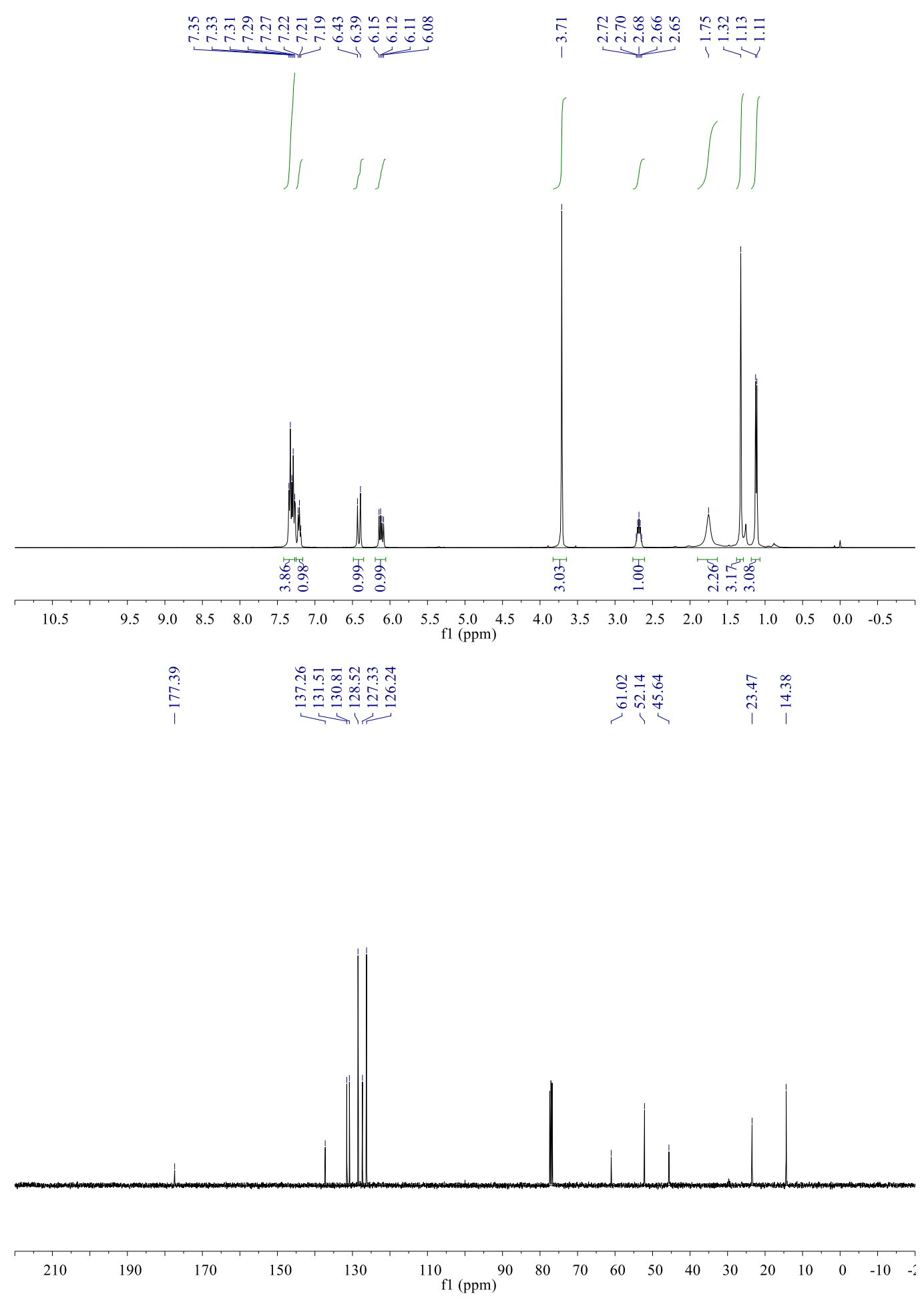
<smiles>COC(=O)C(C)C(N)C(C)C=Cc1ccc(C)cc1</smiles>

\section{$(2 S, 3 R)-3 \mathbf{b a}$}
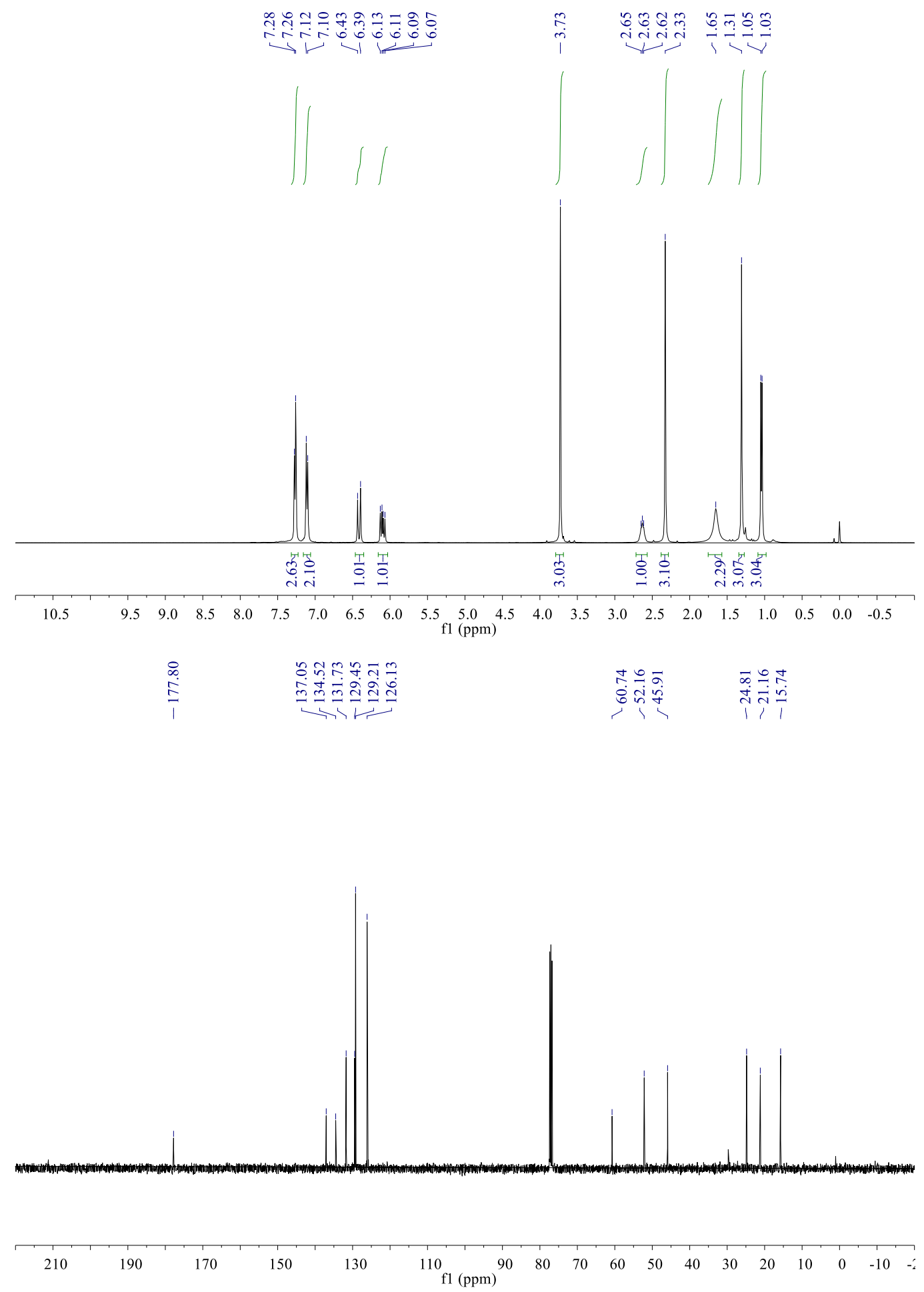
<smiles>COC(=O)C(C)C(C)C=Cc1cccc(C)c1</smiles>
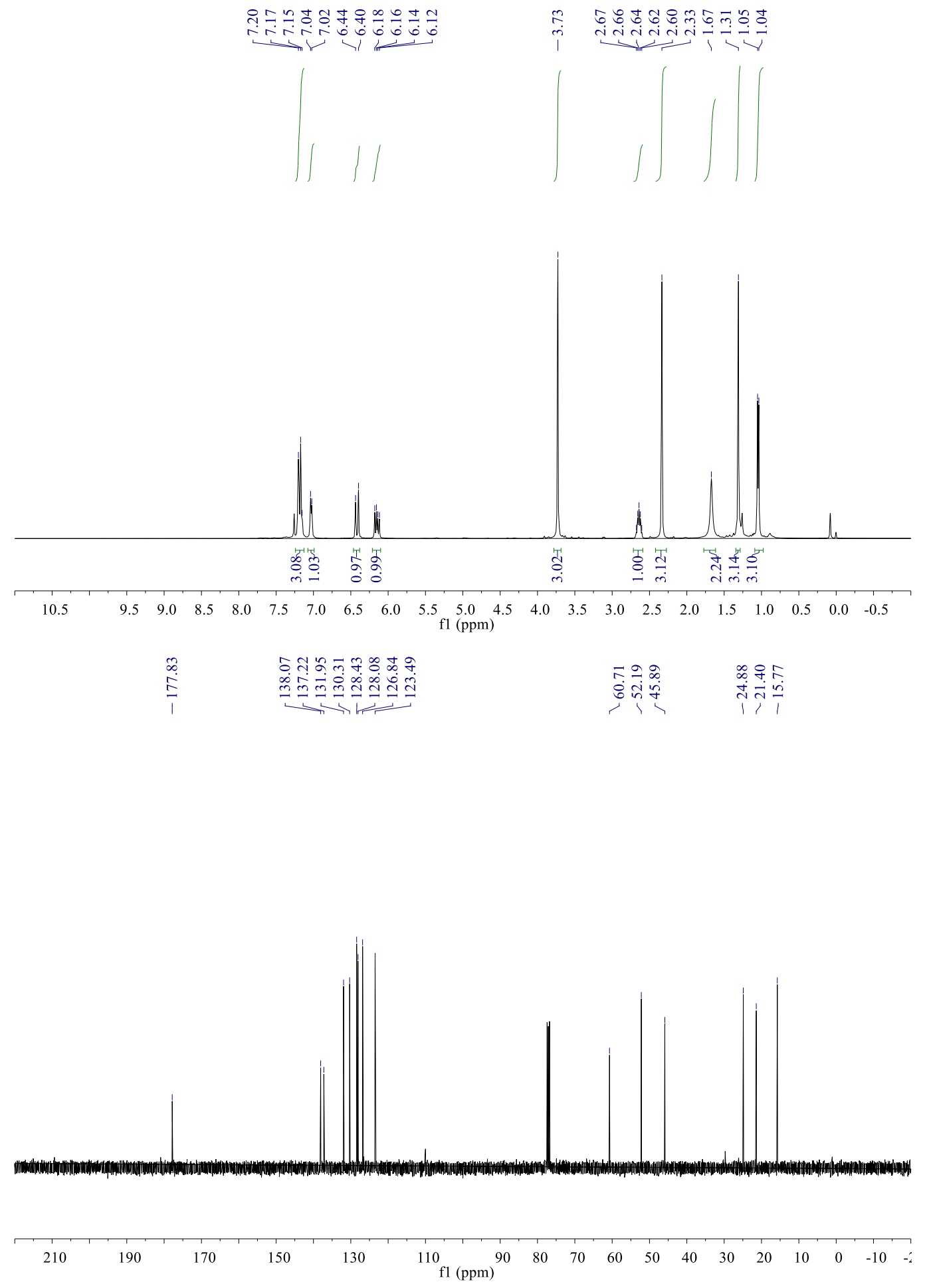

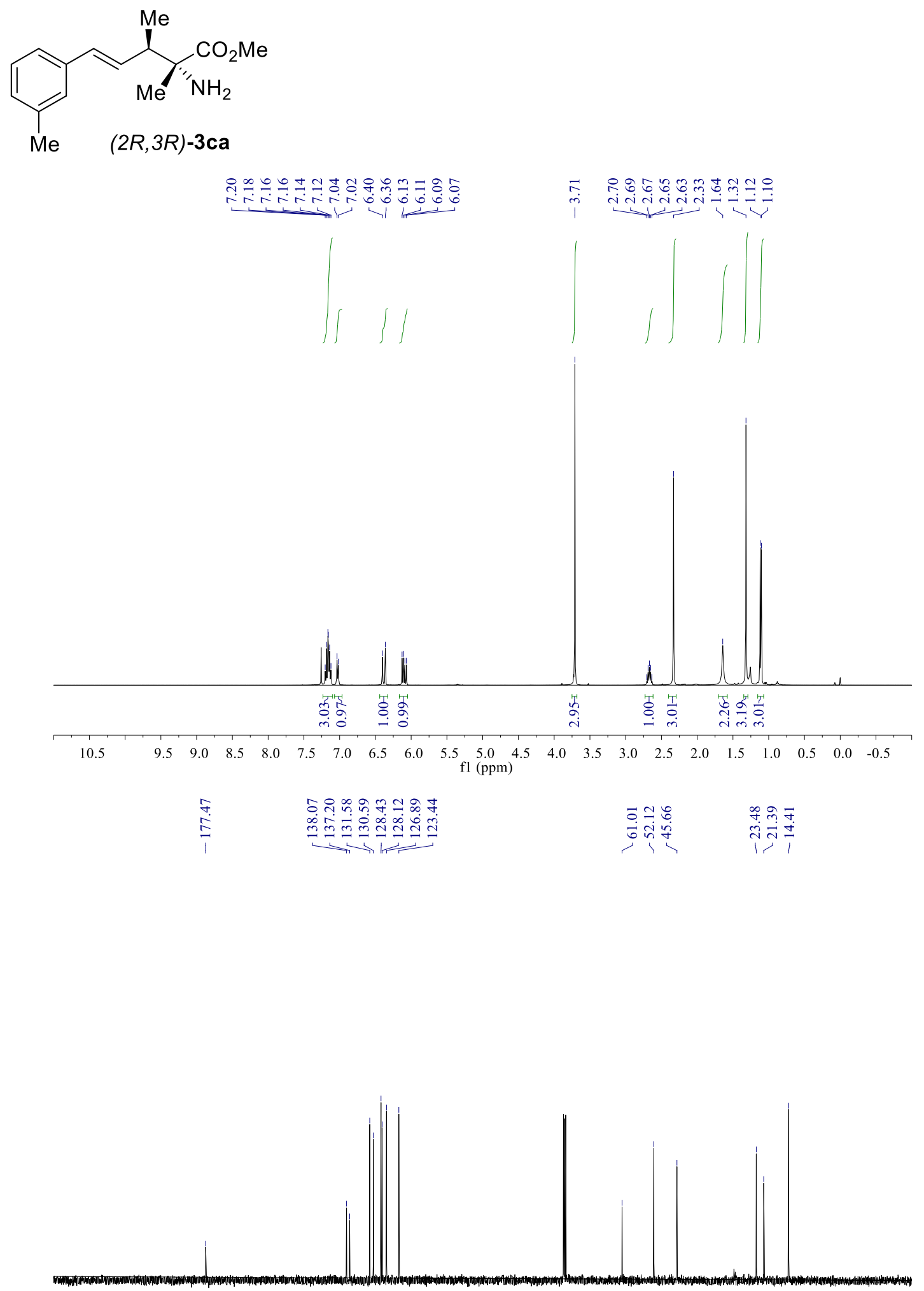

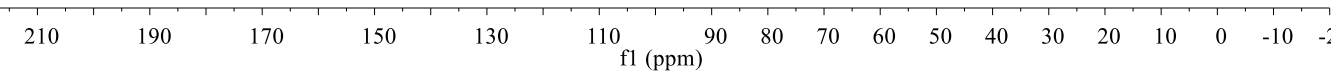


<smiles>COC(=O)[C@](C)(N)[C@H](C)/C=C/c1ccccc1F</smiles>

(2S, 3R)-3da
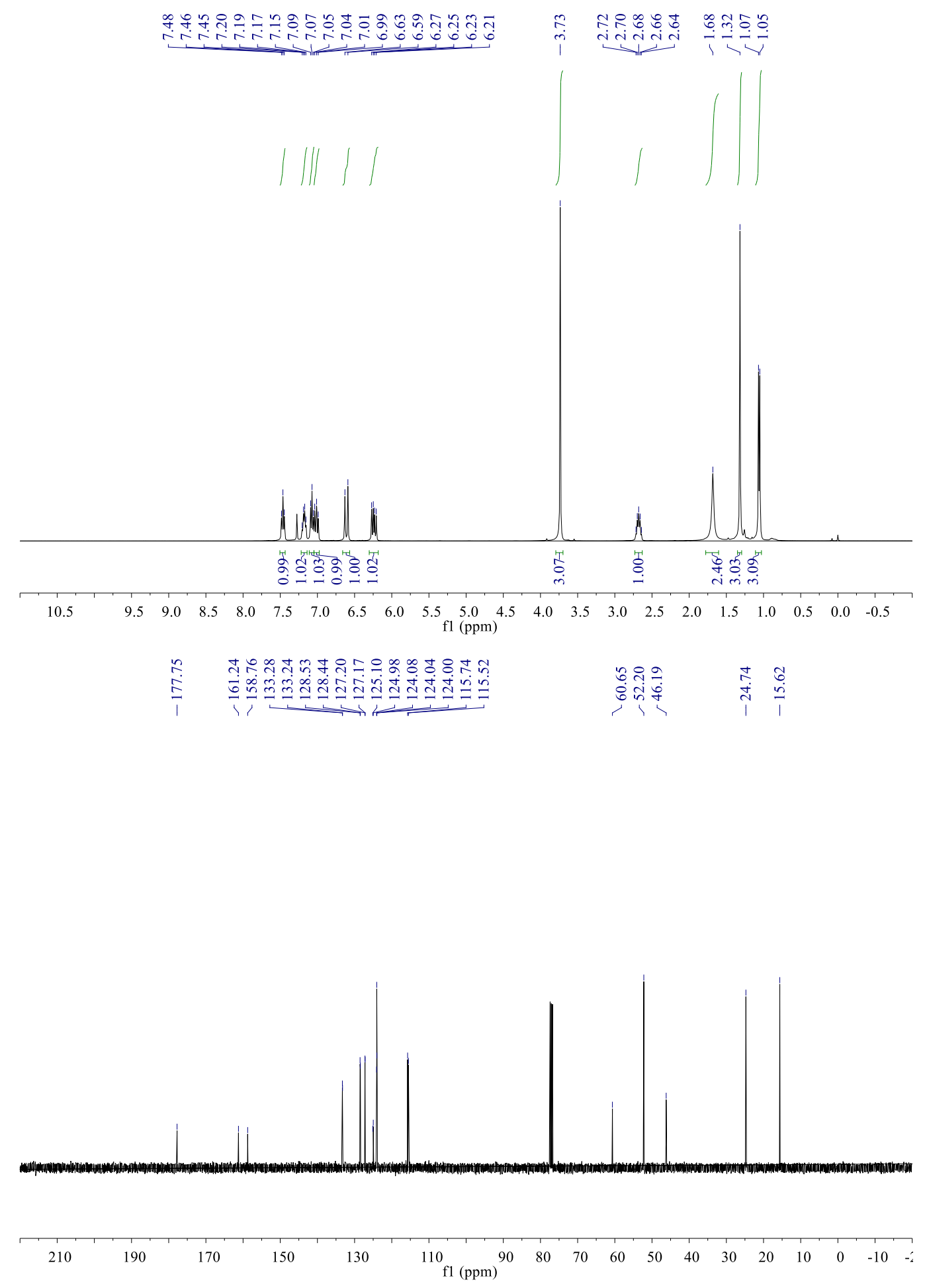


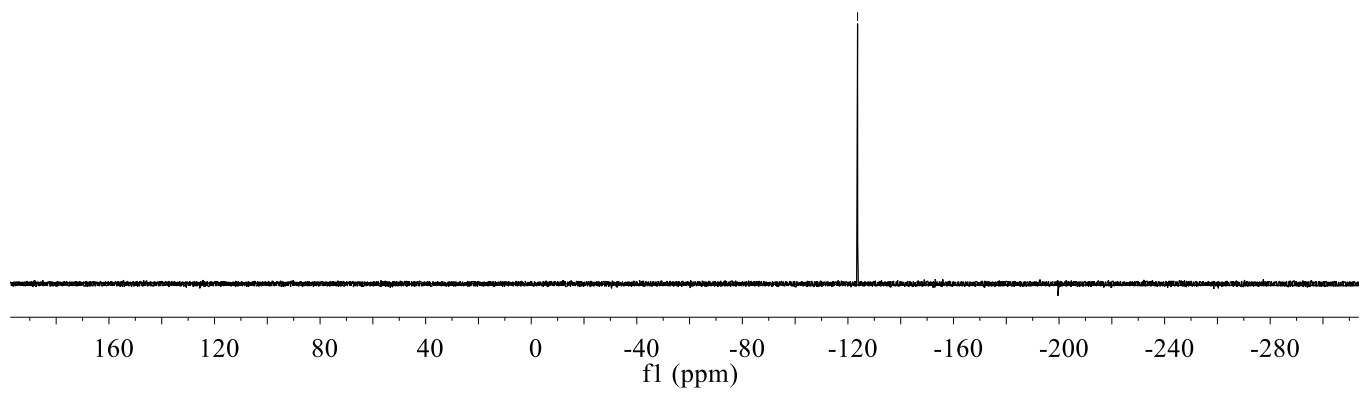<smiles>COC(=O)[C@](C)(N)[C@@H](C)/C=C/c1ccccc1F</smiles>

\section{(2R,3R)-3da}

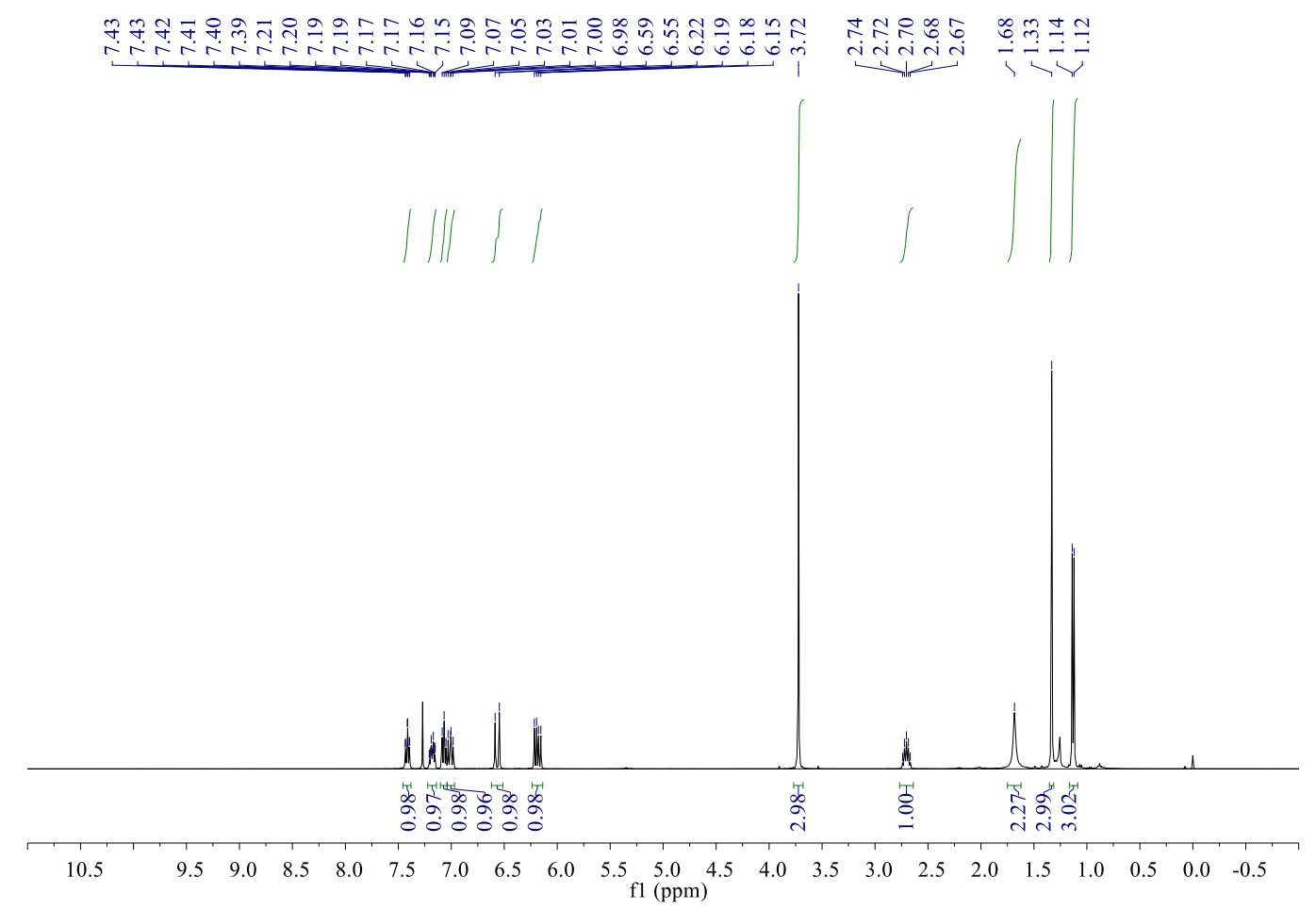



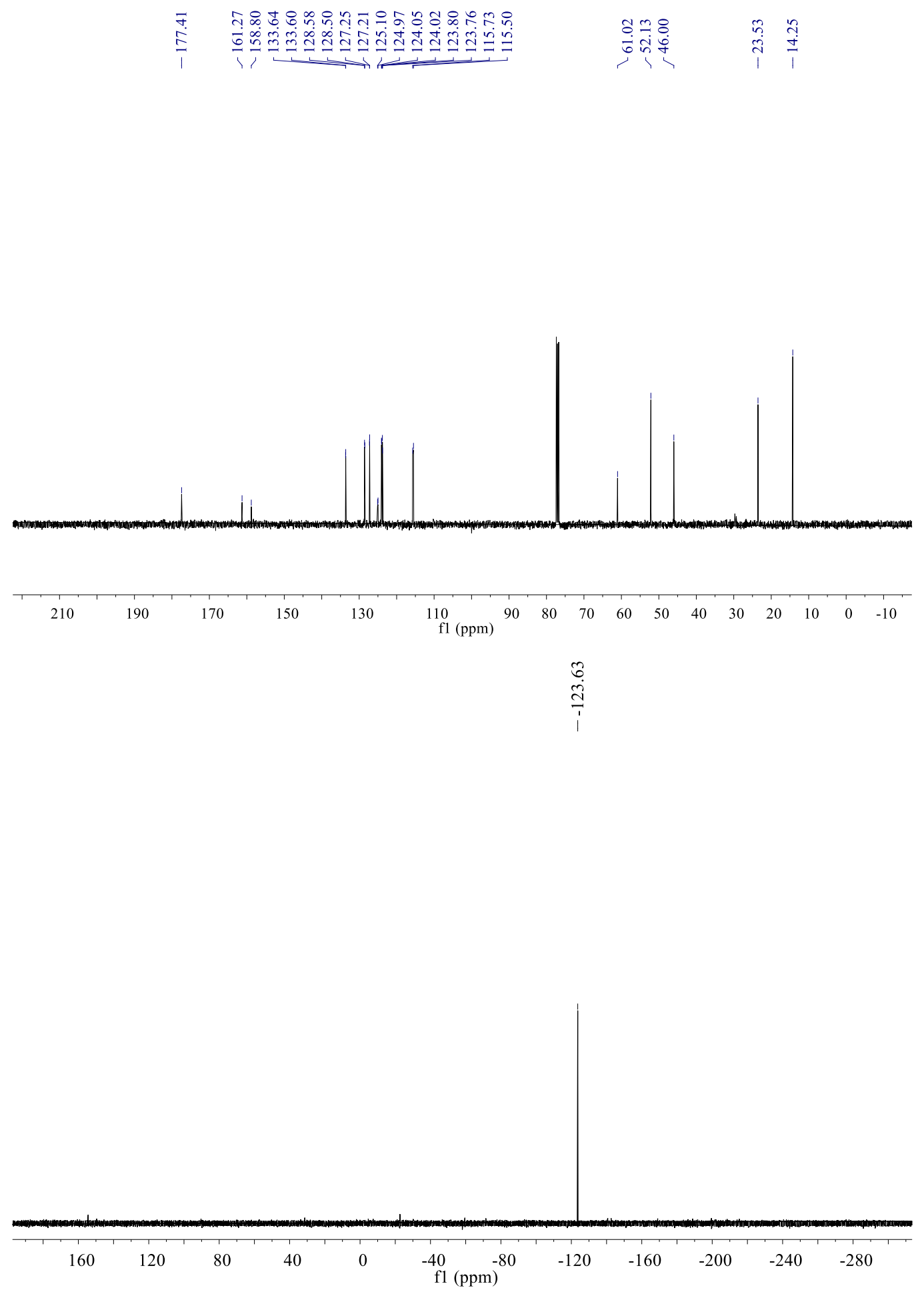
<smiles>COC(=O)C(C)C(C)C(C)C=Cc1cccc(F)c1</smiles>
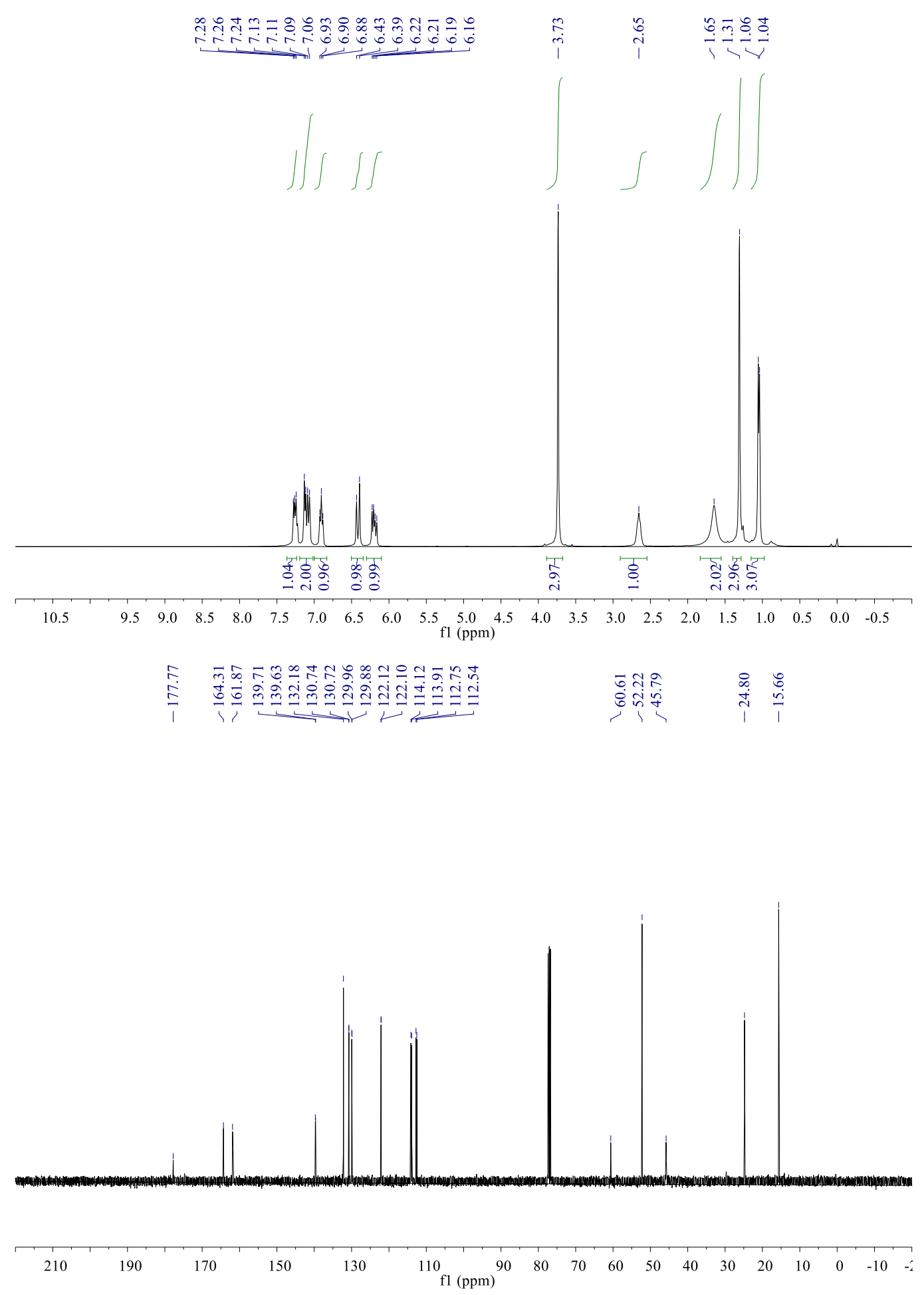


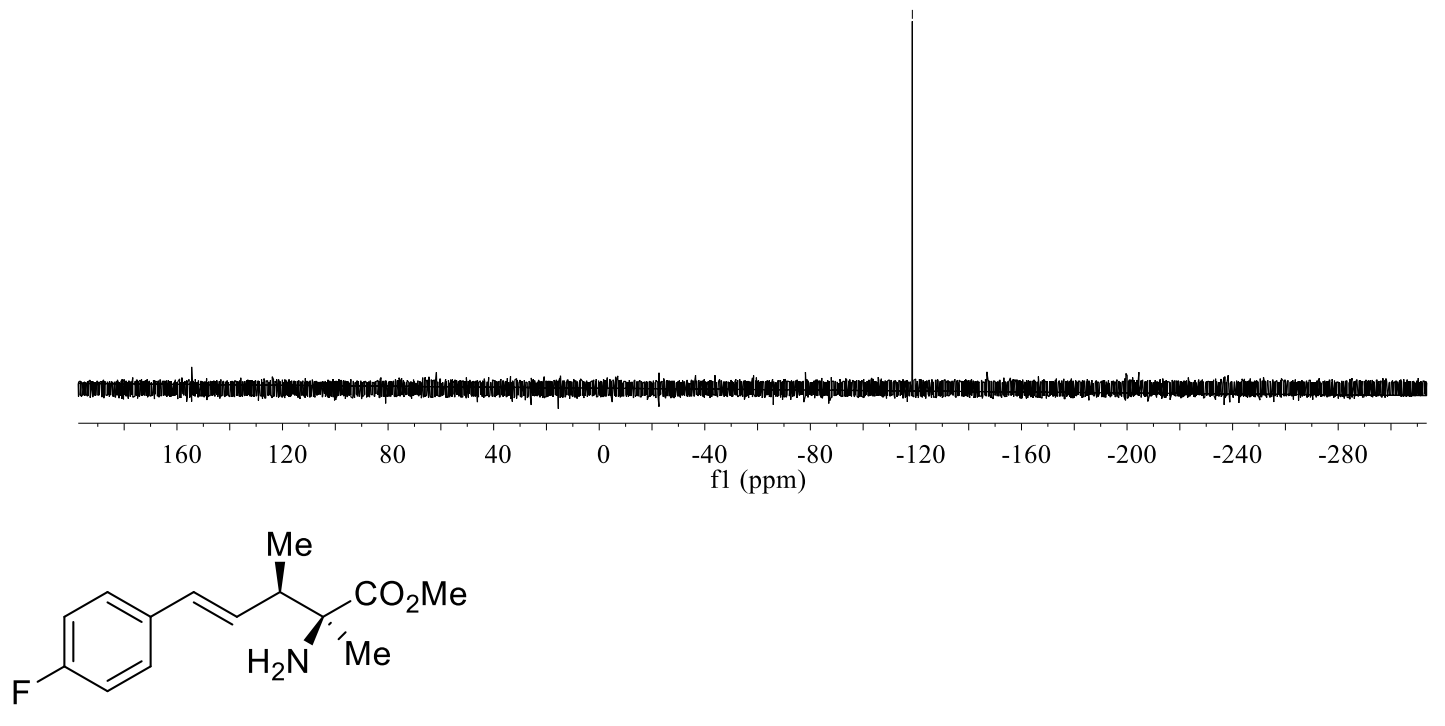

(2S, 3R)-3fa

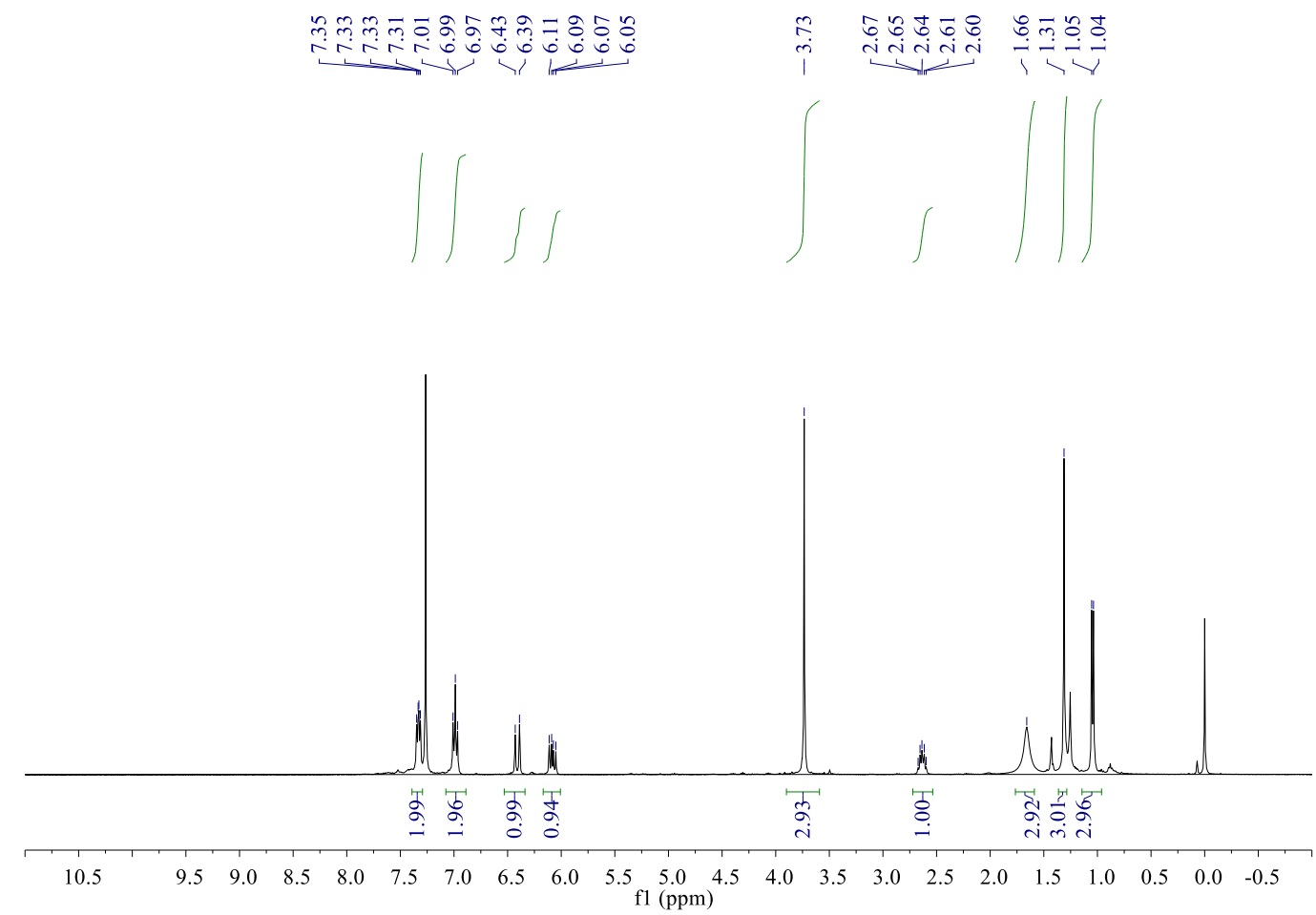




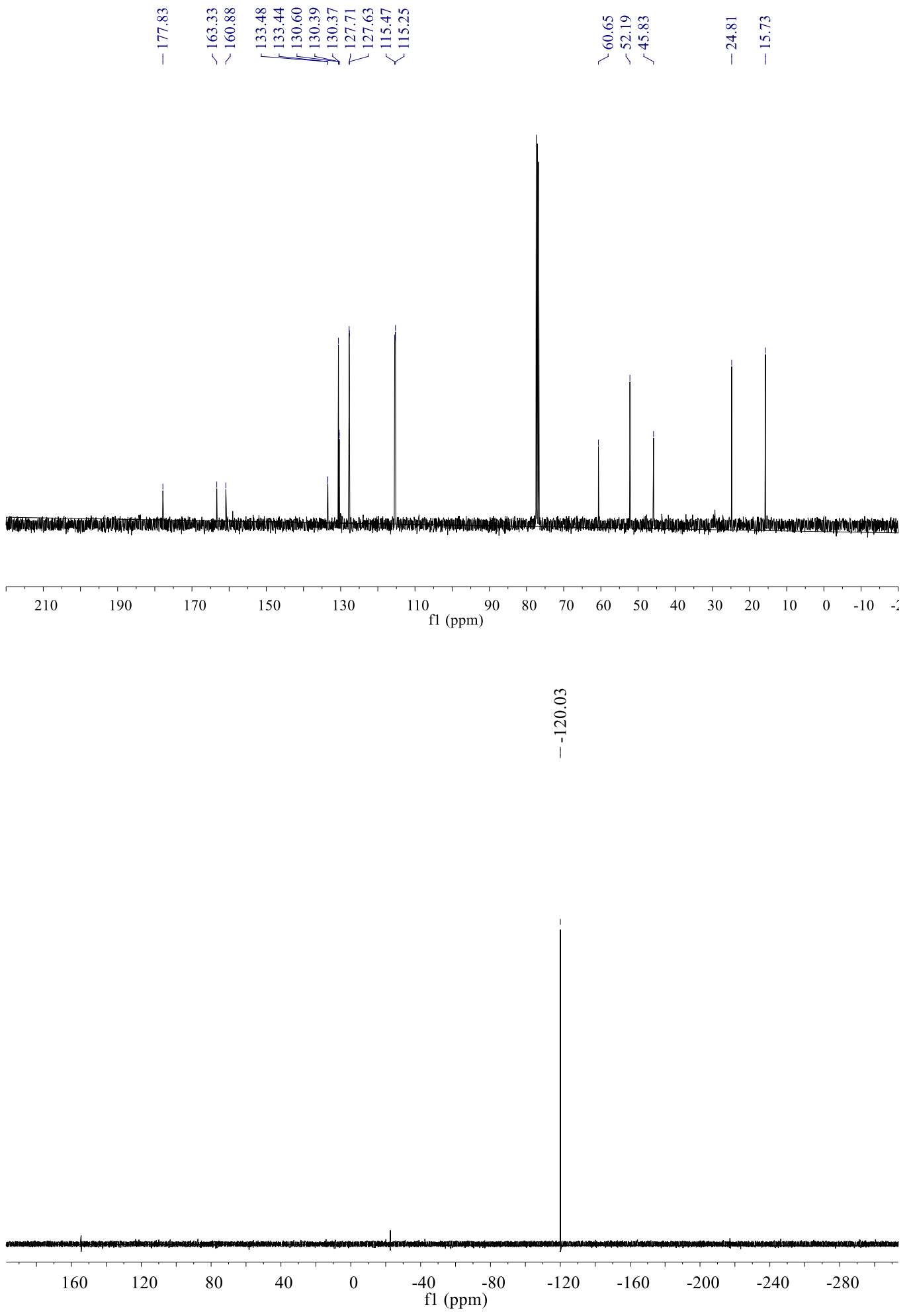


<smiles>COC(=O)[C@](C)(N)[C@@H](C)/C=C/c1ccc(F)cc1</smiles>
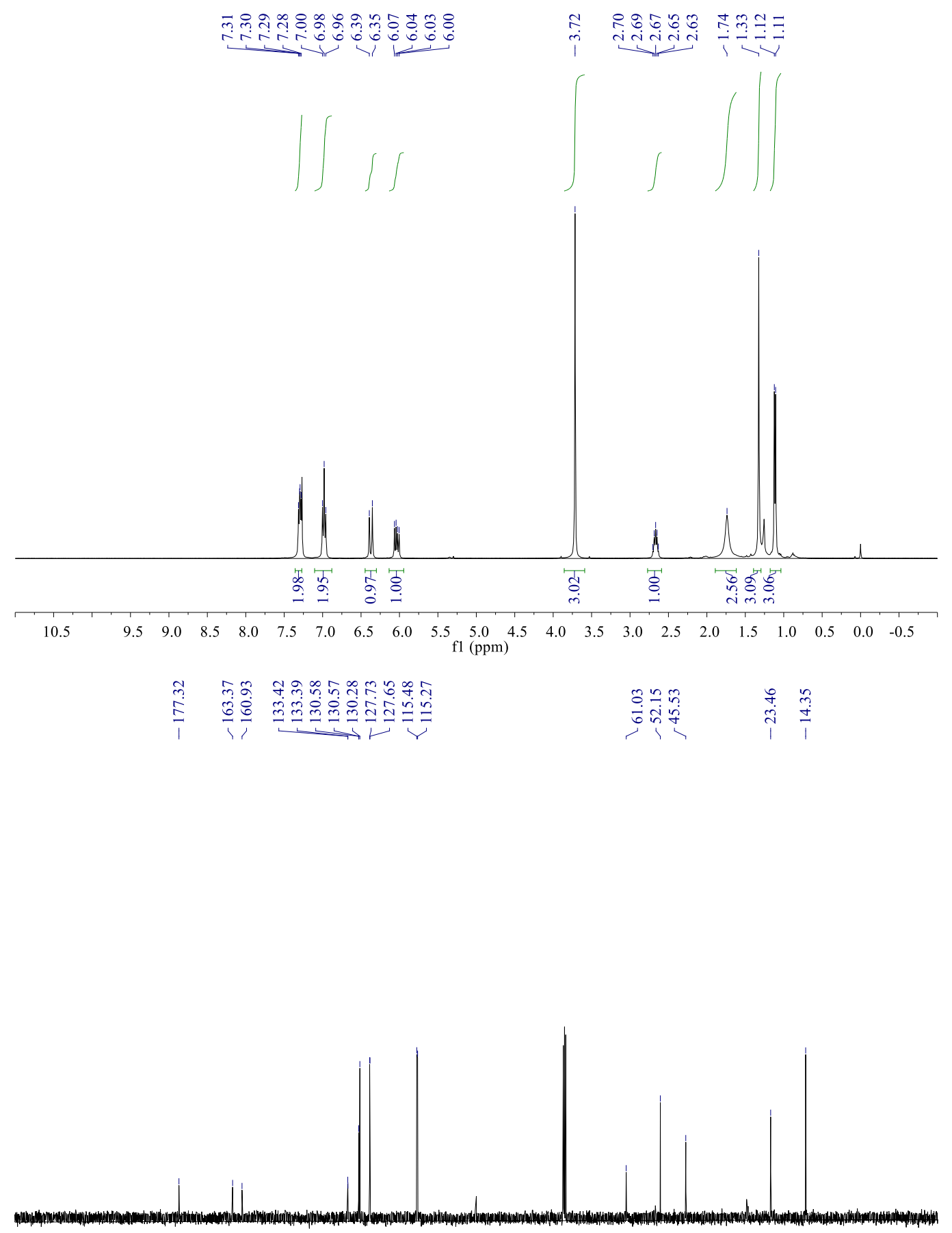

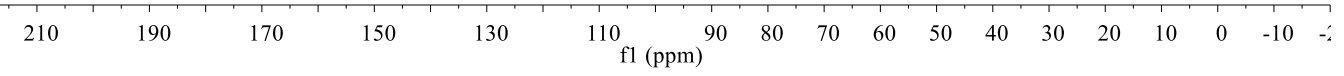




\section{$\frac{a}{a}$}

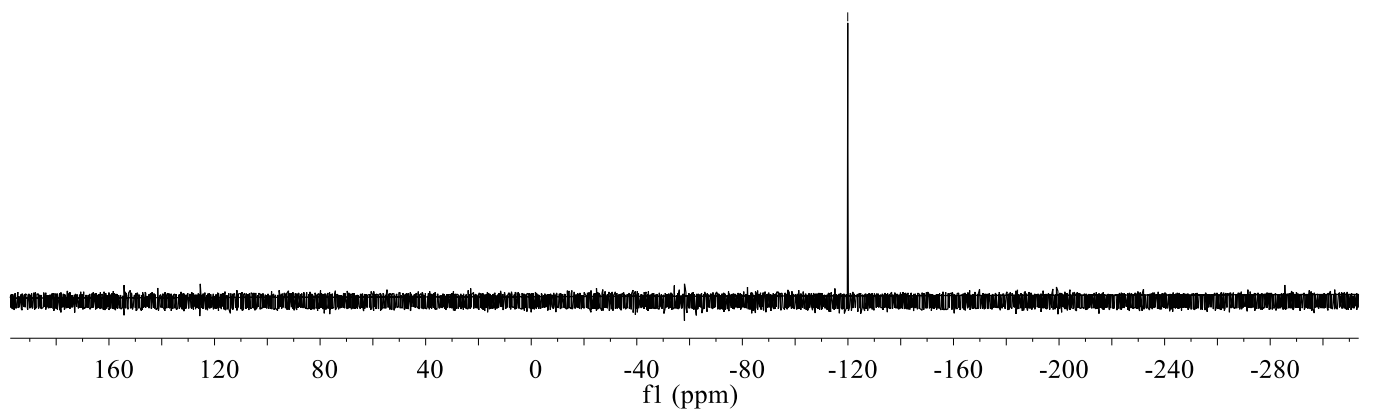

(c)

(2S, 3R)-3ga
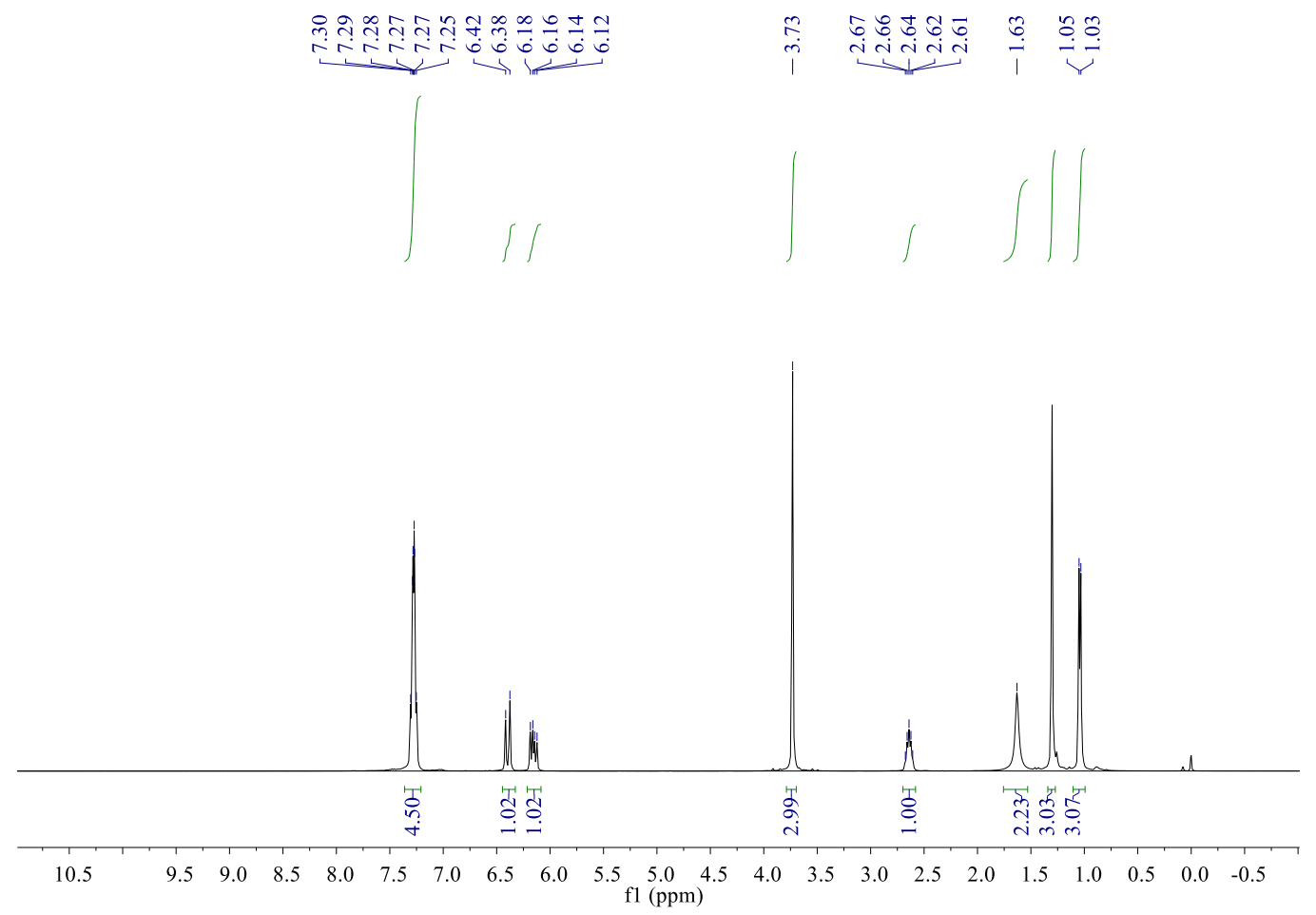

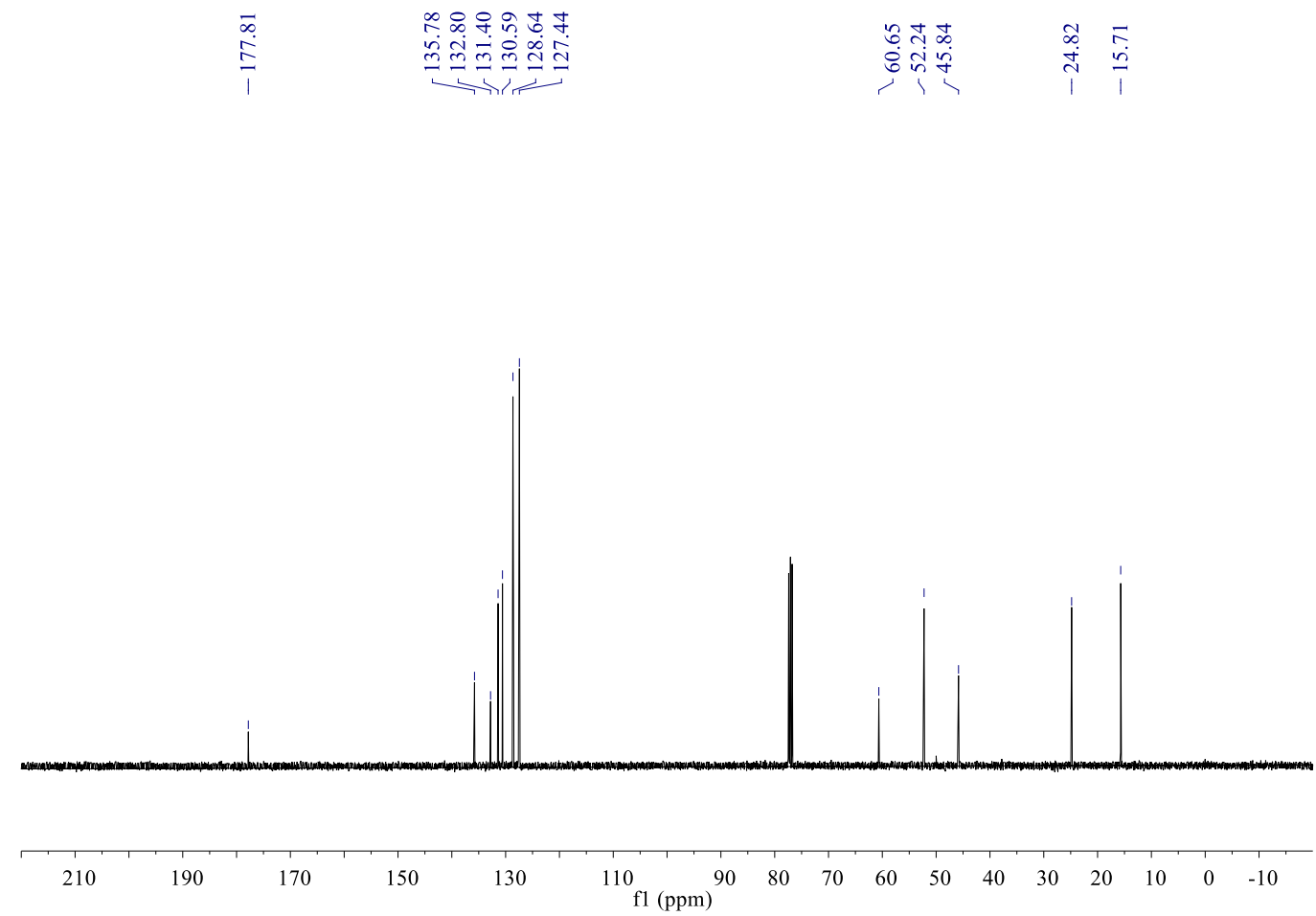<smiles>COC(=O)[C@H](N)[C@H](C)/C=C/c1ccc(C(F)(F)F)cc1</smiles>

\section{(2S, 3R)-3ha}

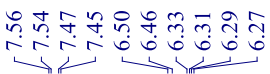

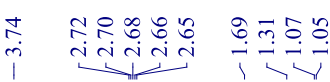
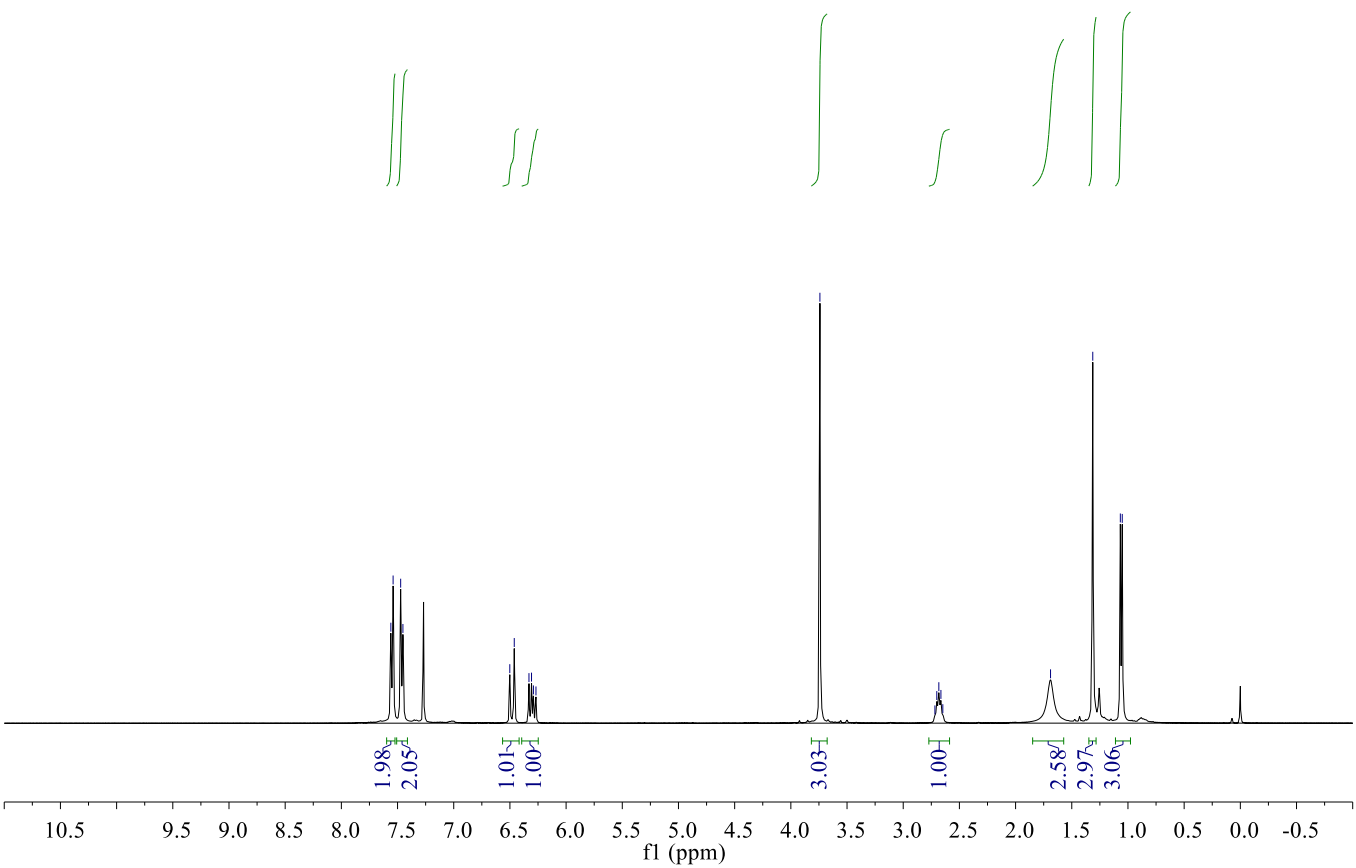

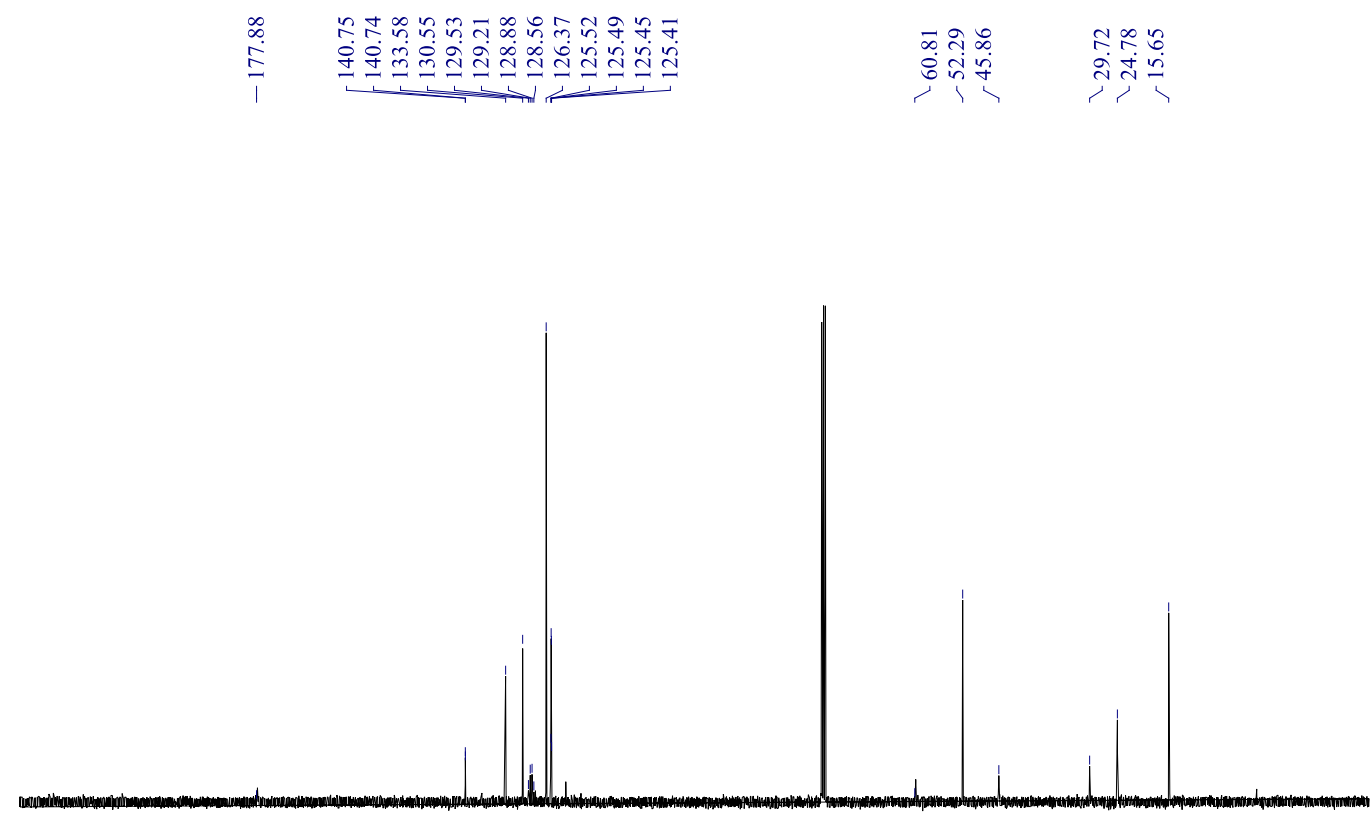

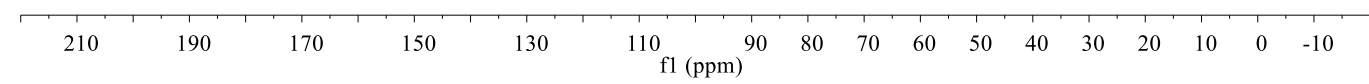

p9

$\frac{2}{8}$

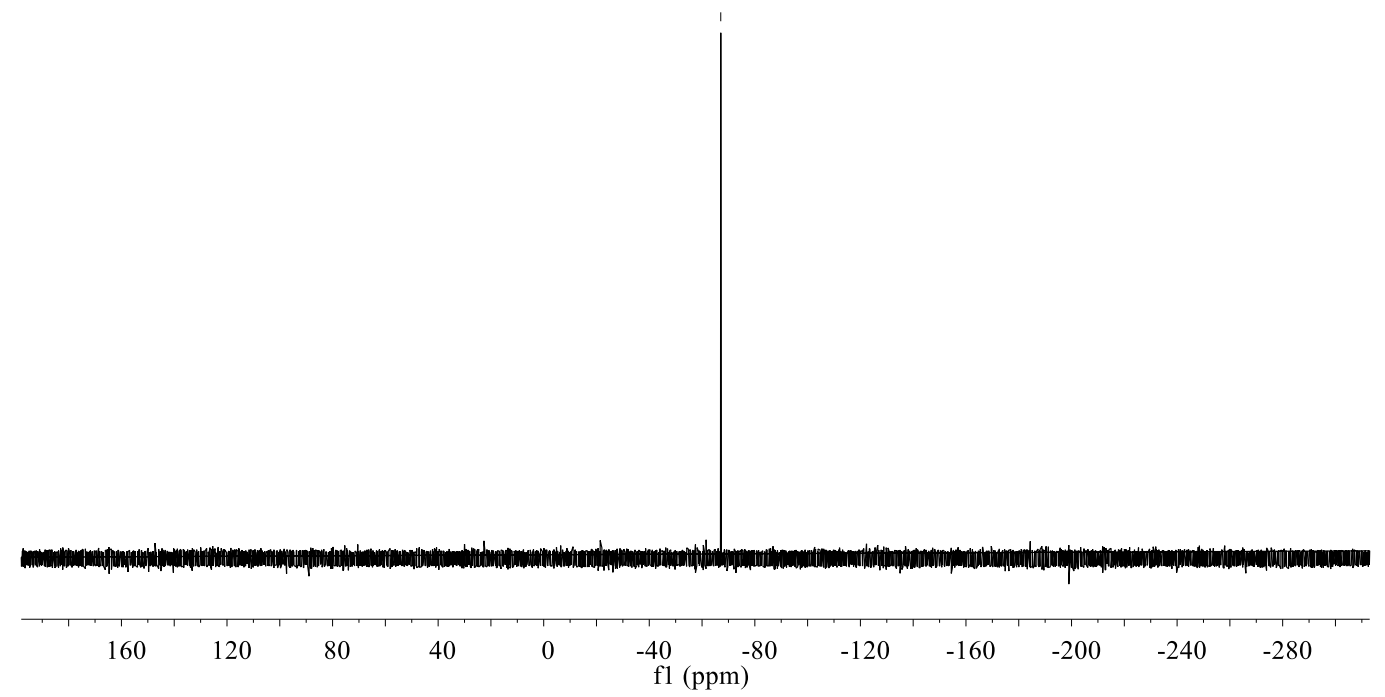


<smiles>[M]C(/C=C/c1ccc(OC)cc1)C(C)(N)C(C)=O</smiles>

$(2 S, 3 R)-3 \mathbf{i a}$
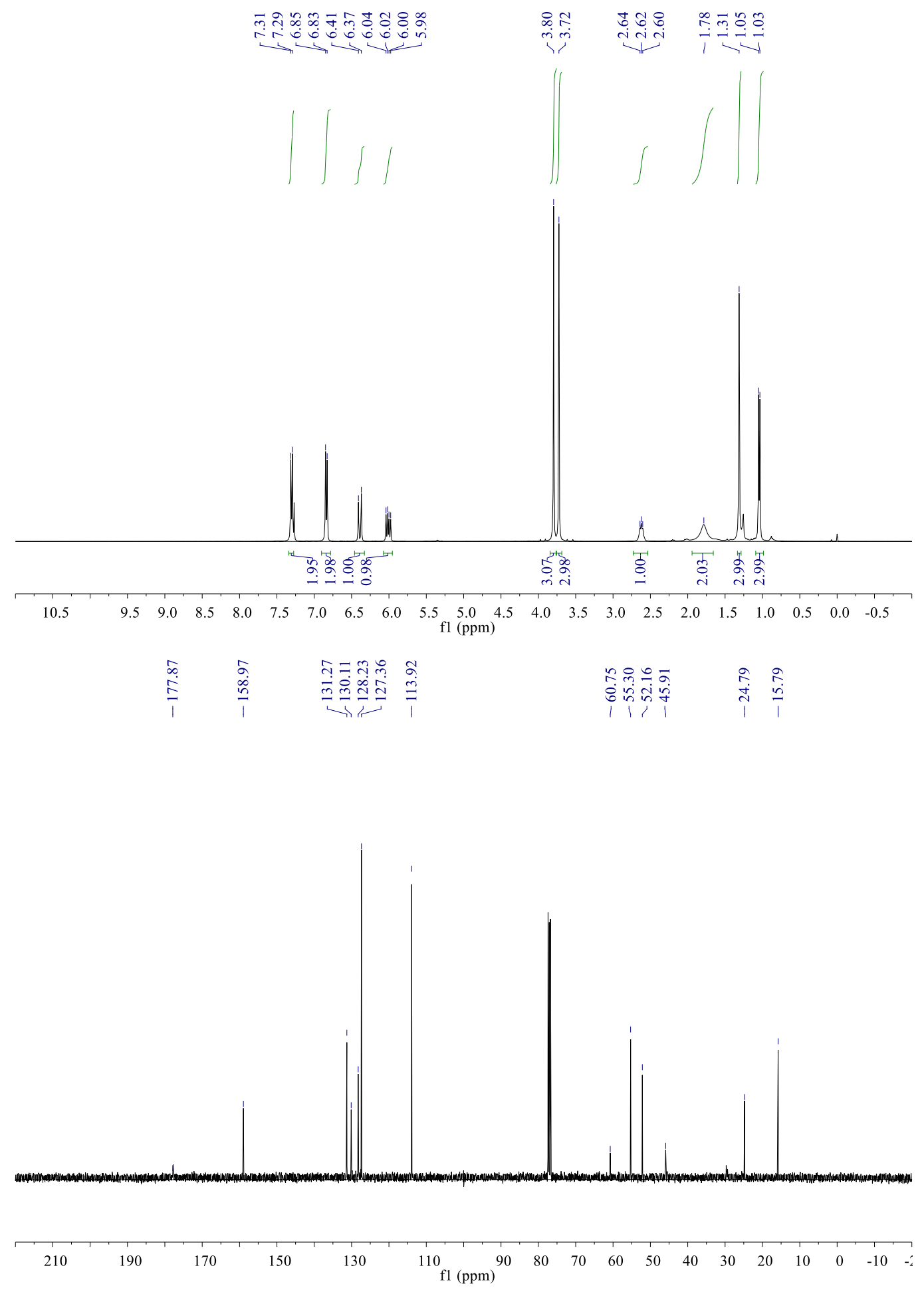

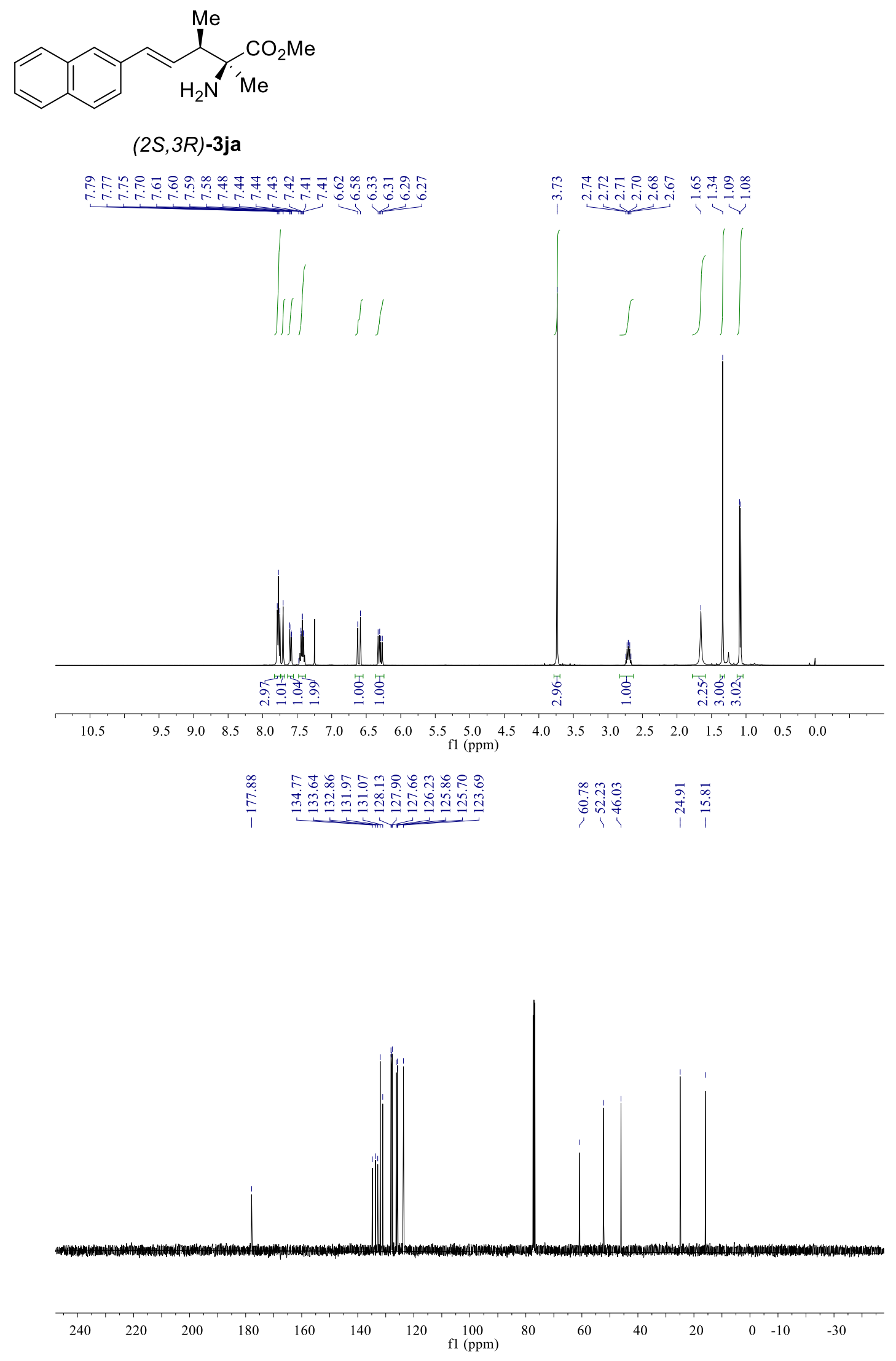

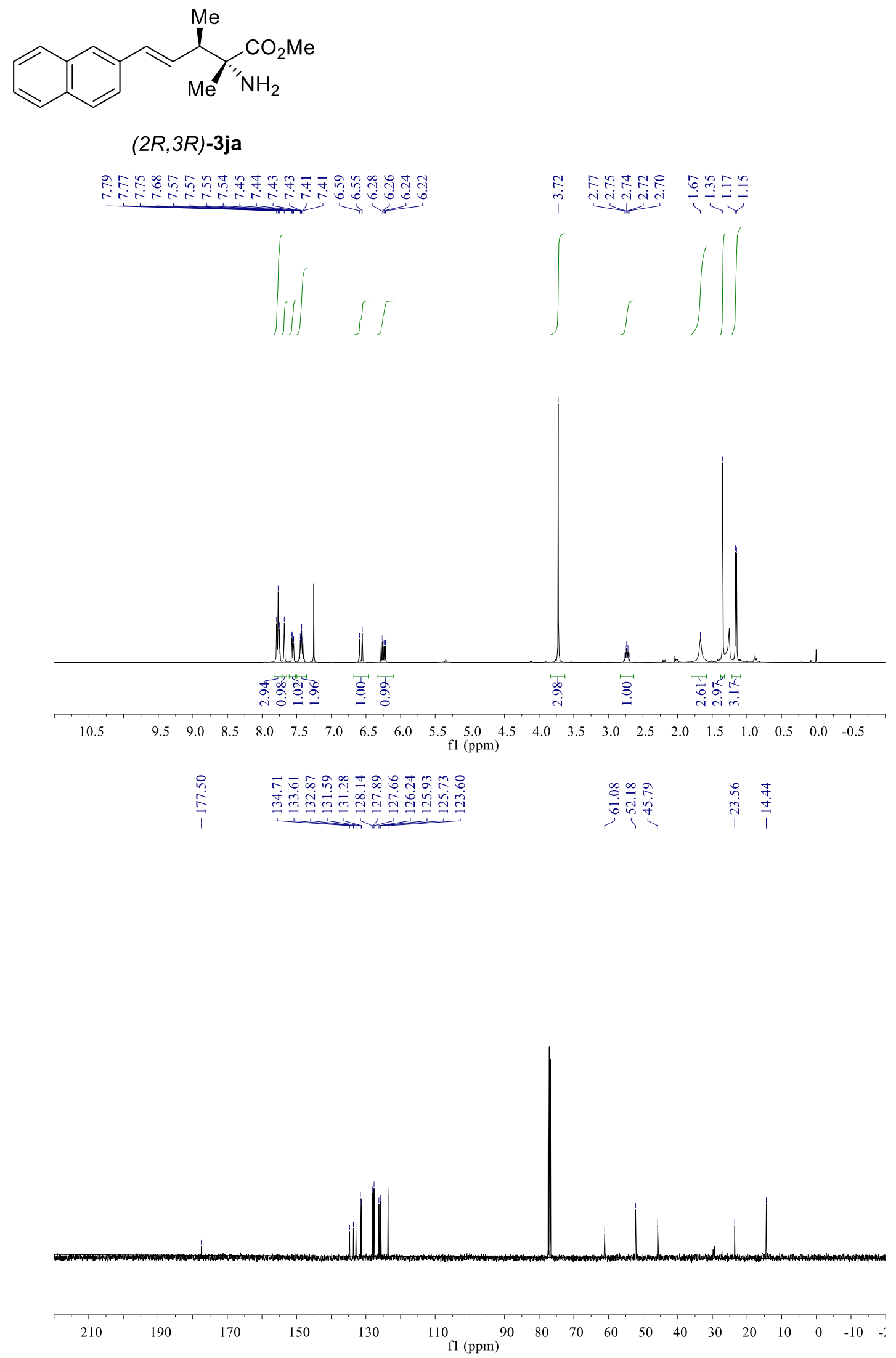


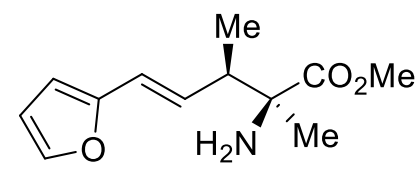

(2S, 3R)-3ka
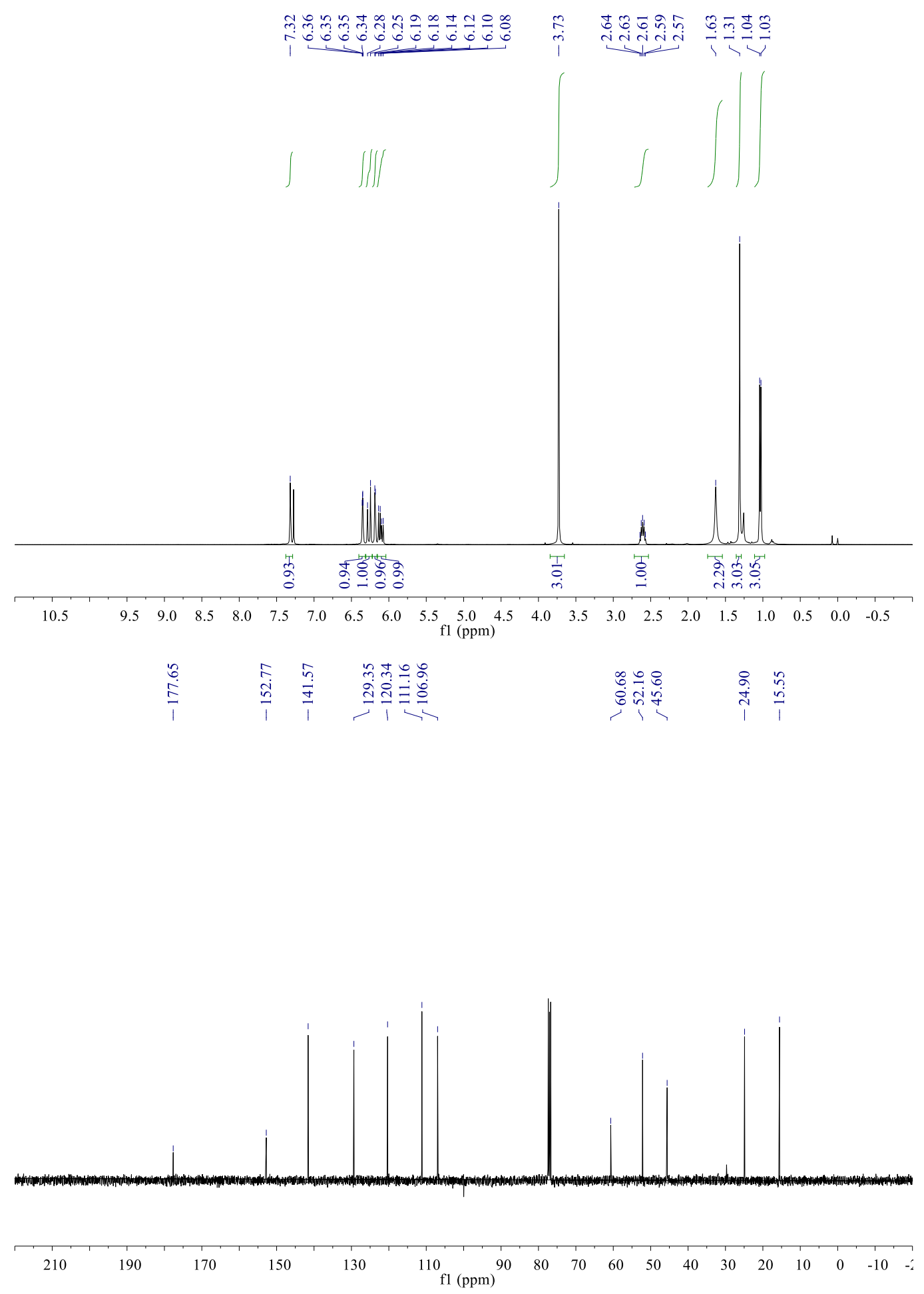


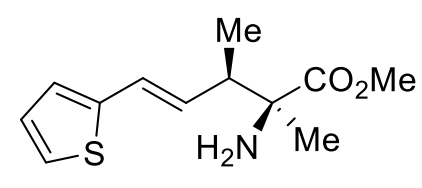

(2S, 3R)-3la
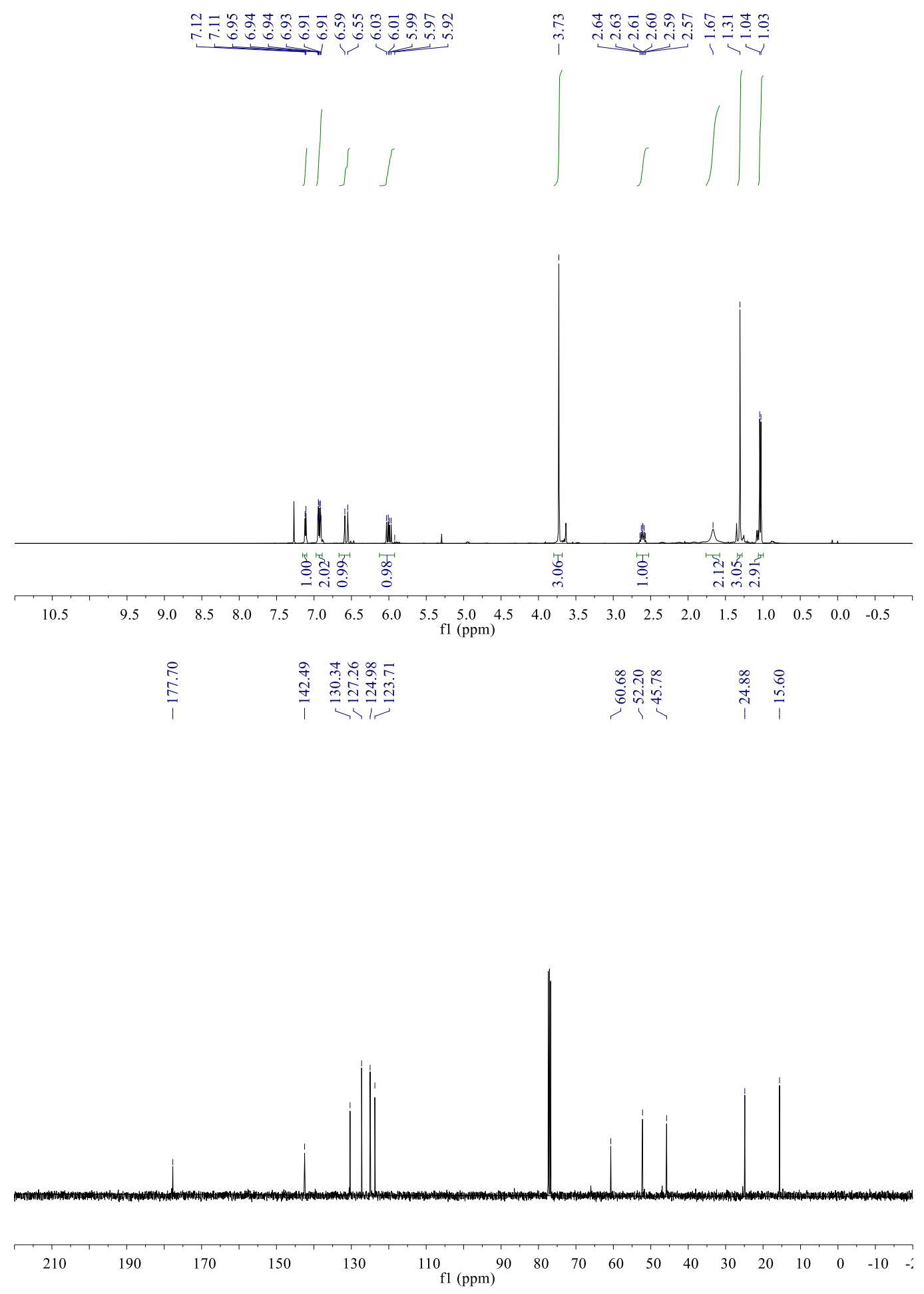

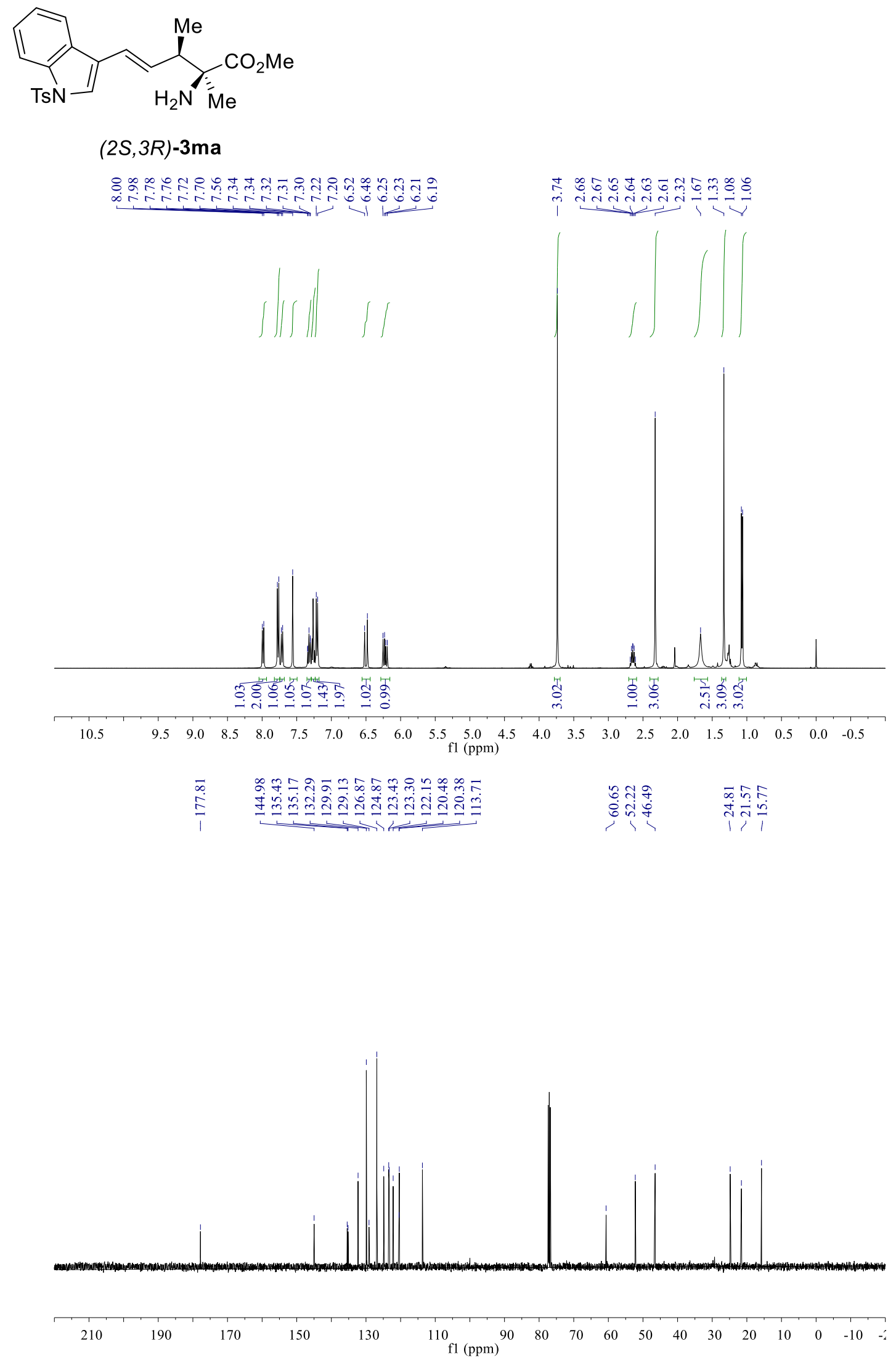
<smiles>COC(=O)C(C)(N)C(C)C=CCCO[Co]</smiles>

(2S, 3R)-3na
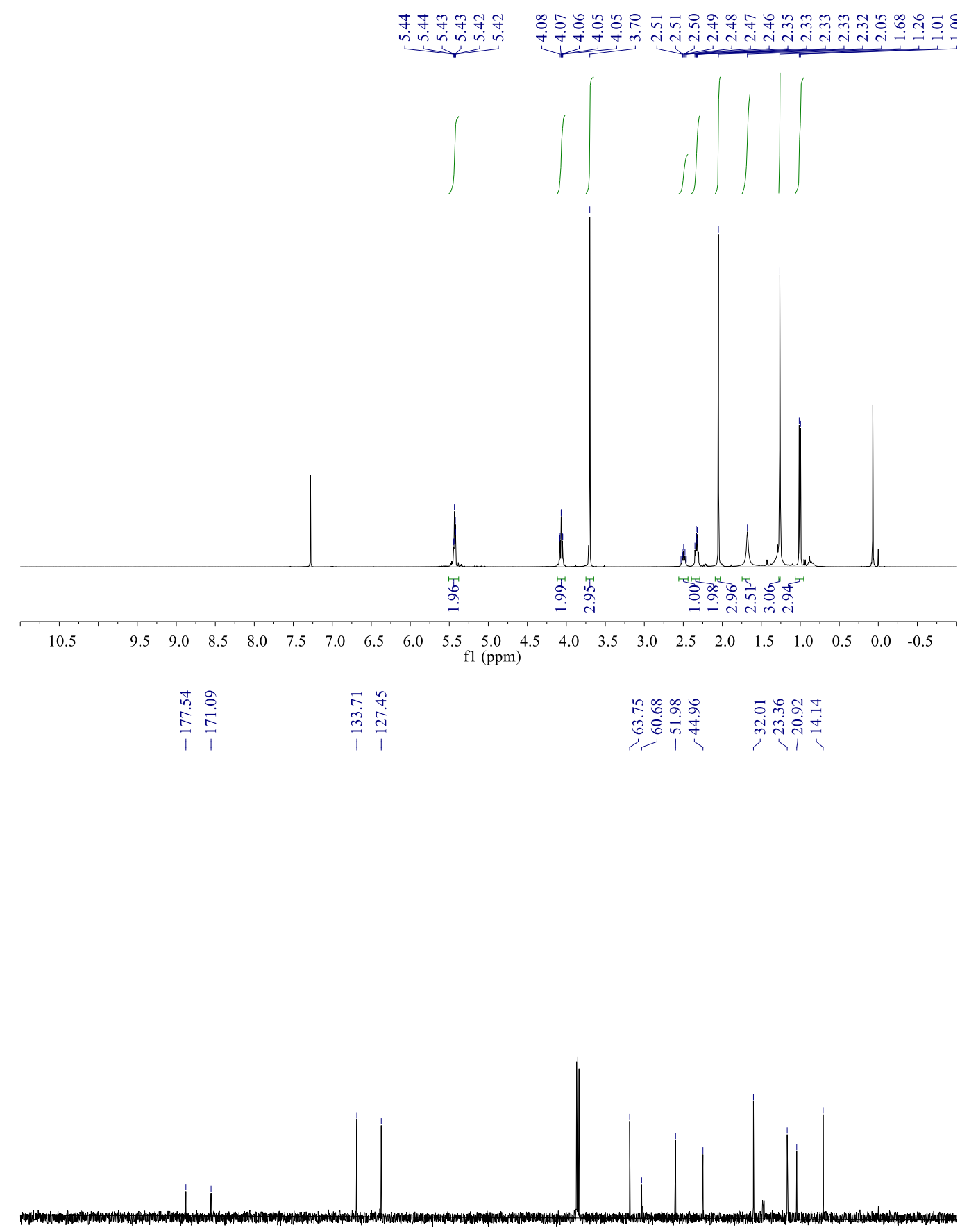

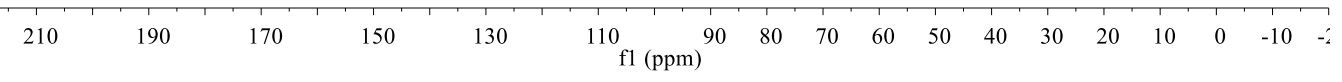


$\overbrace{\mathrm{H}_{2} \mathrm{~N}}^{\mathrm{Ct}} \mathrm{CO}_{\mathrm{Me}}^{\mathrm{Me}}$

(2S, 3R)-3oa
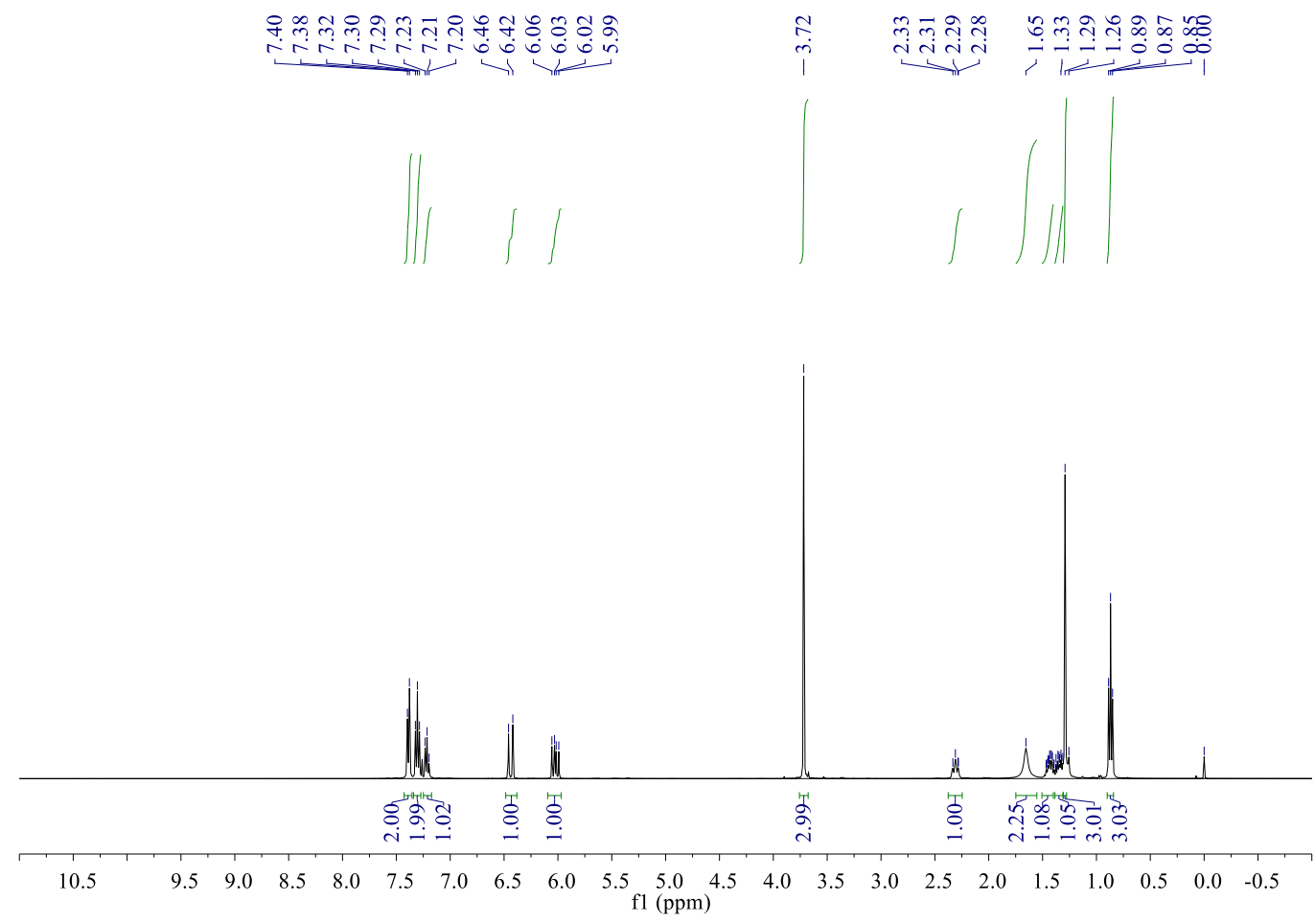

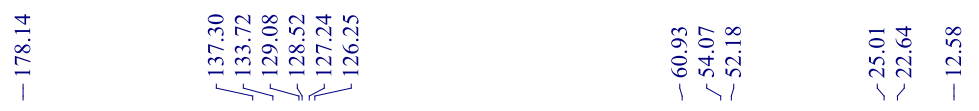
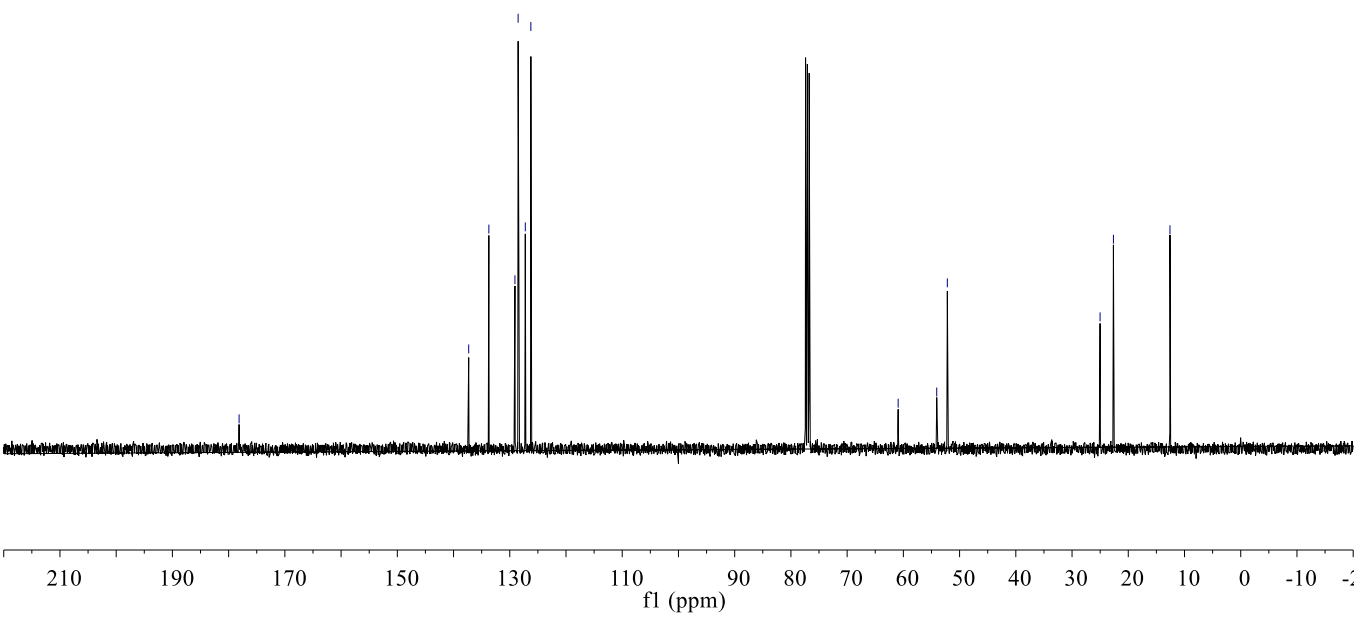
<smiles>CC[C@H](/C=C/c1ccccc1)[C@@](C)(N)C(C)=O</smiles>

\section{(2R,3R)-3oa}
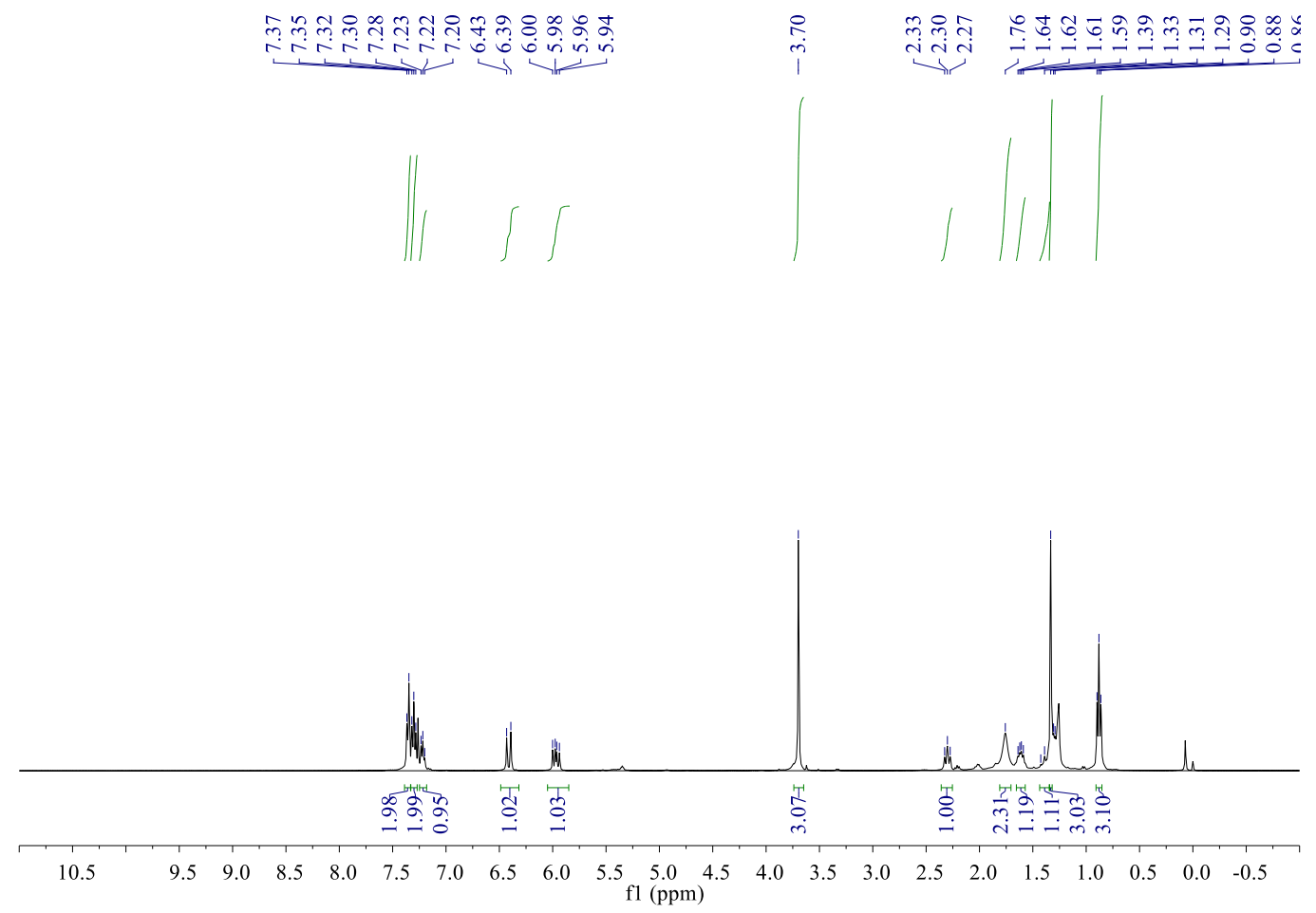

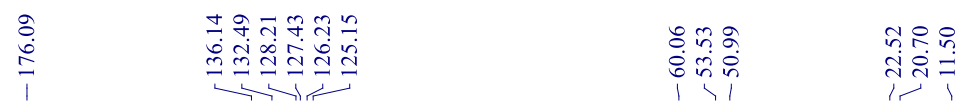
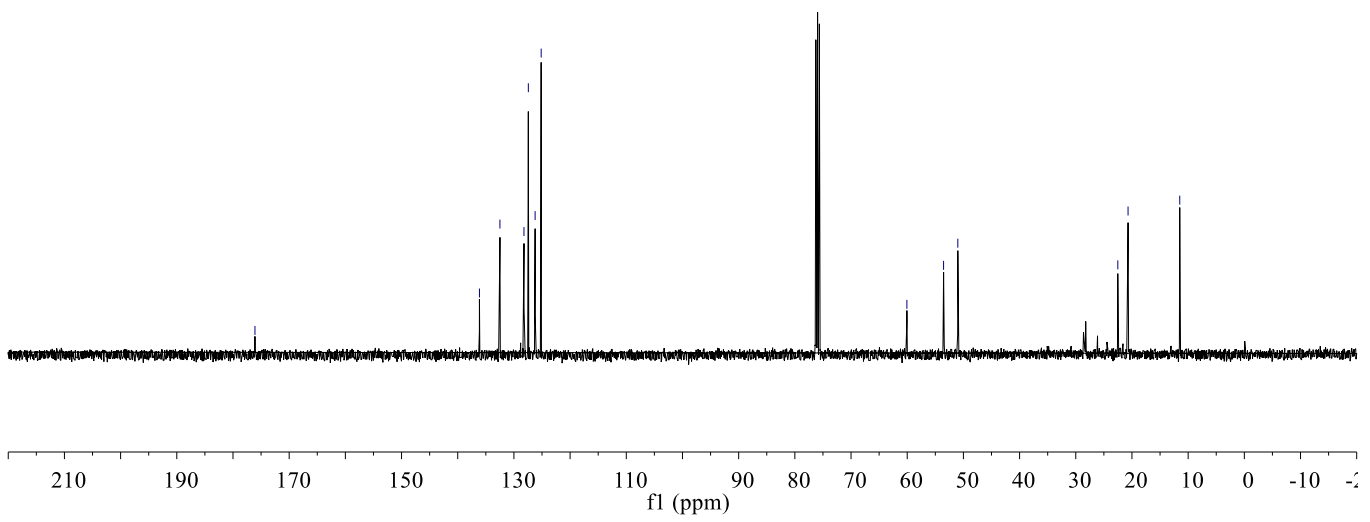
<smiles>CCC(C)C(/C=C/c1ccccc1)C(C)(N)C(C)=O</smiles>

(2S, 3R)-3pa
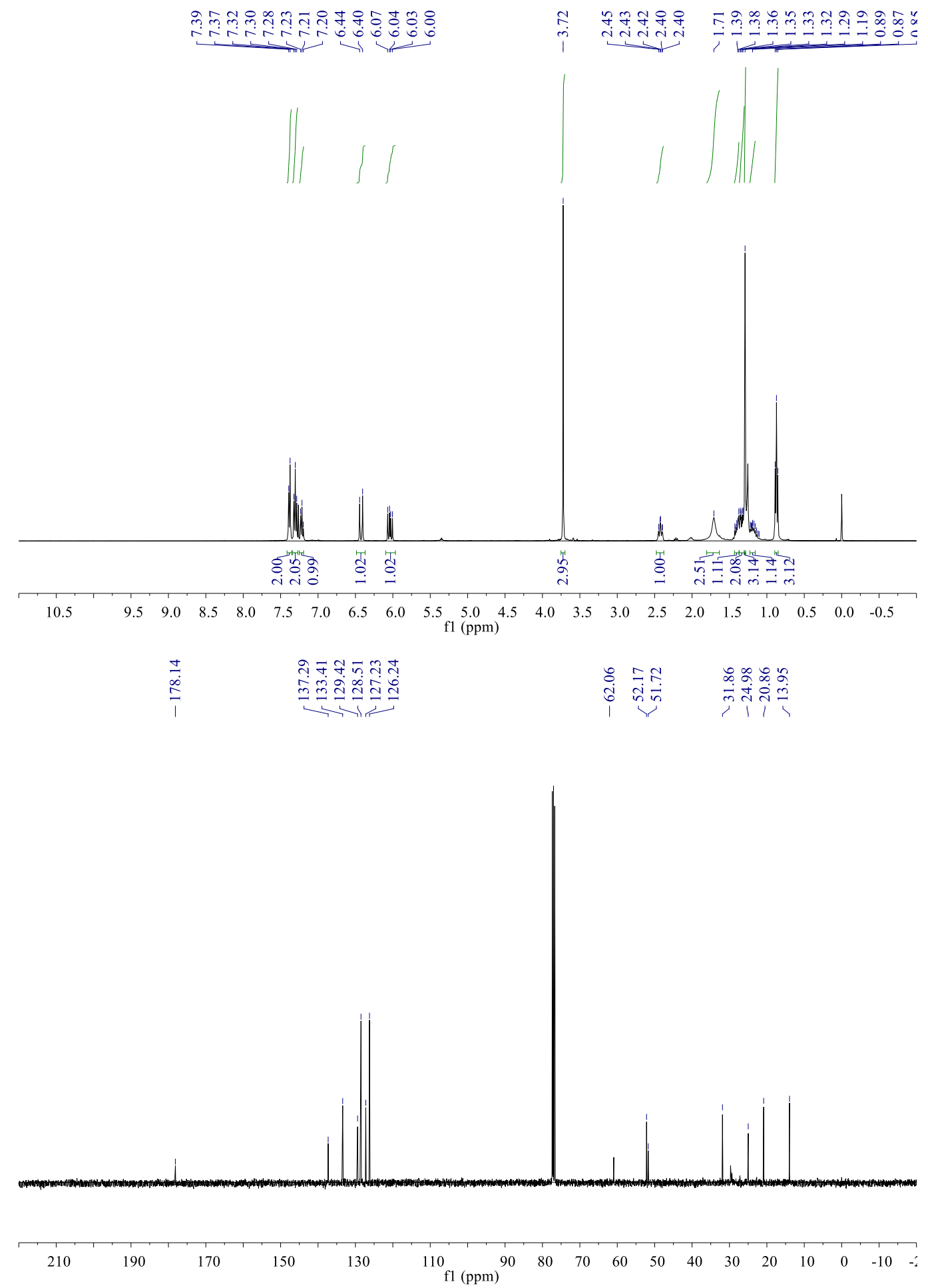


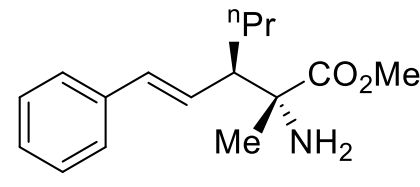

(2R,3R)-3pa inj

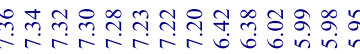

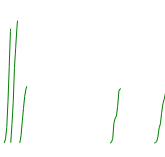

\section{1}

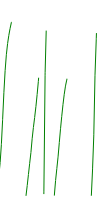

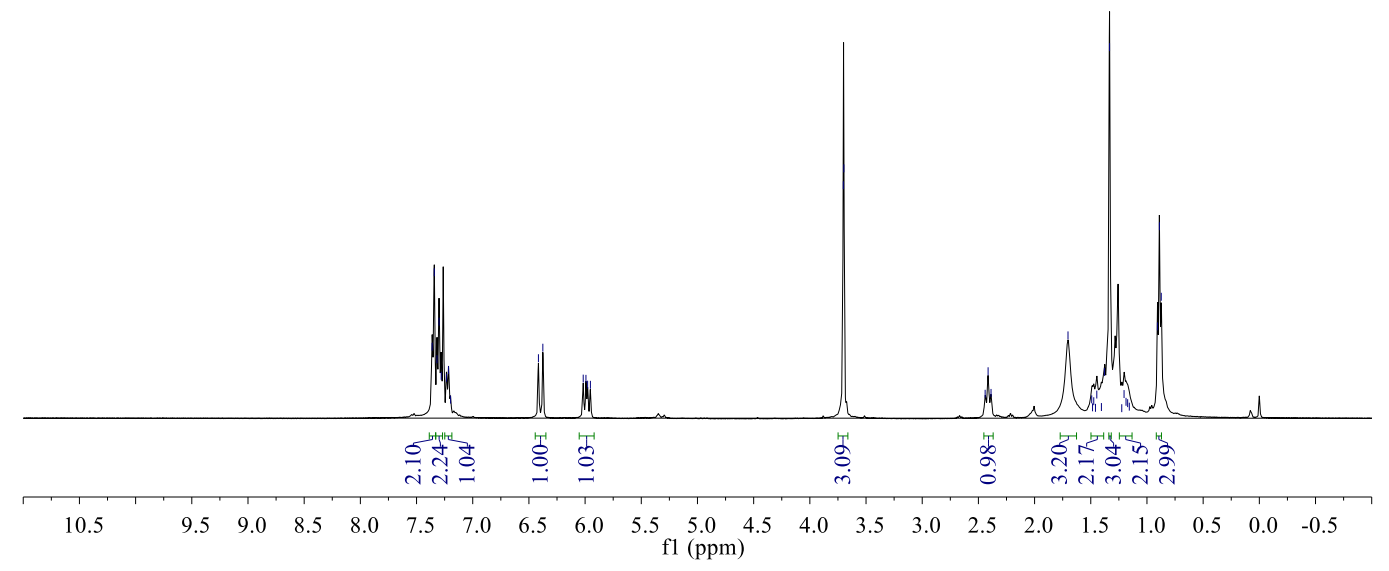

\begin{tabular}{|c|c|}
\hline 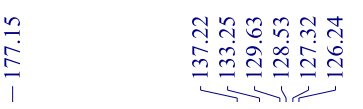 & 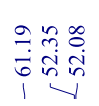 \\
\hline
\end{tabular}
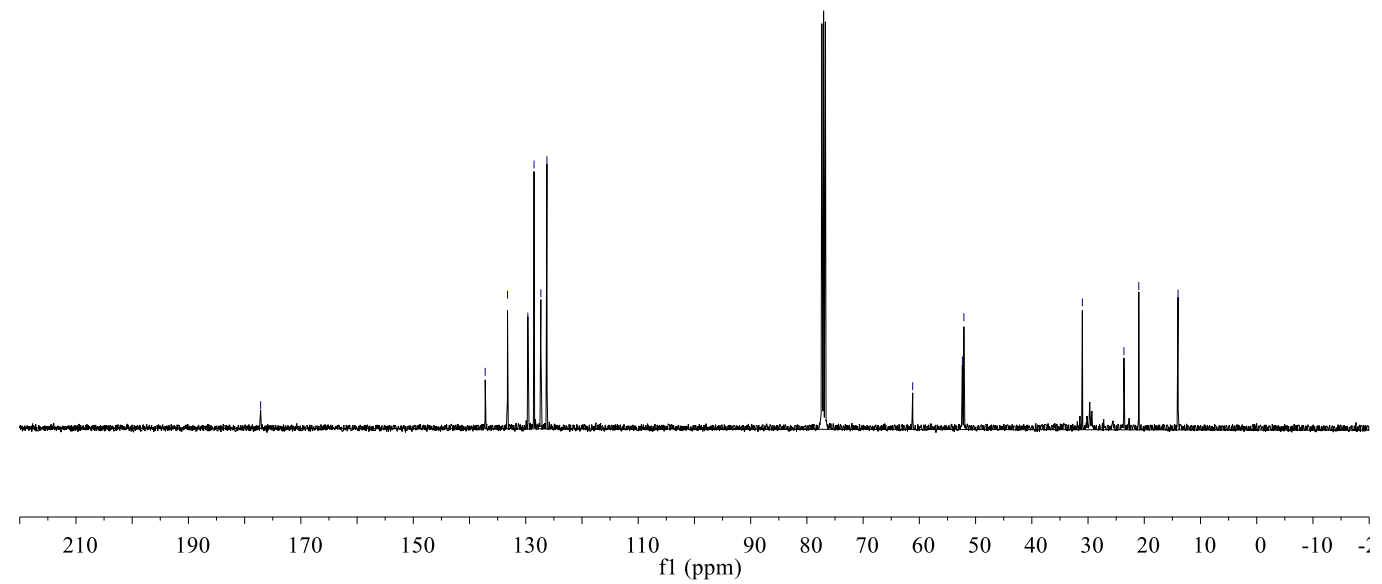
<smiles>COC(=O)C(C)(N)C(/C=C/c1ccccc1)CCCCOCc1ccccc1</smiles>

(2S, 3R)-3qa

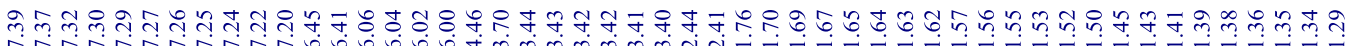
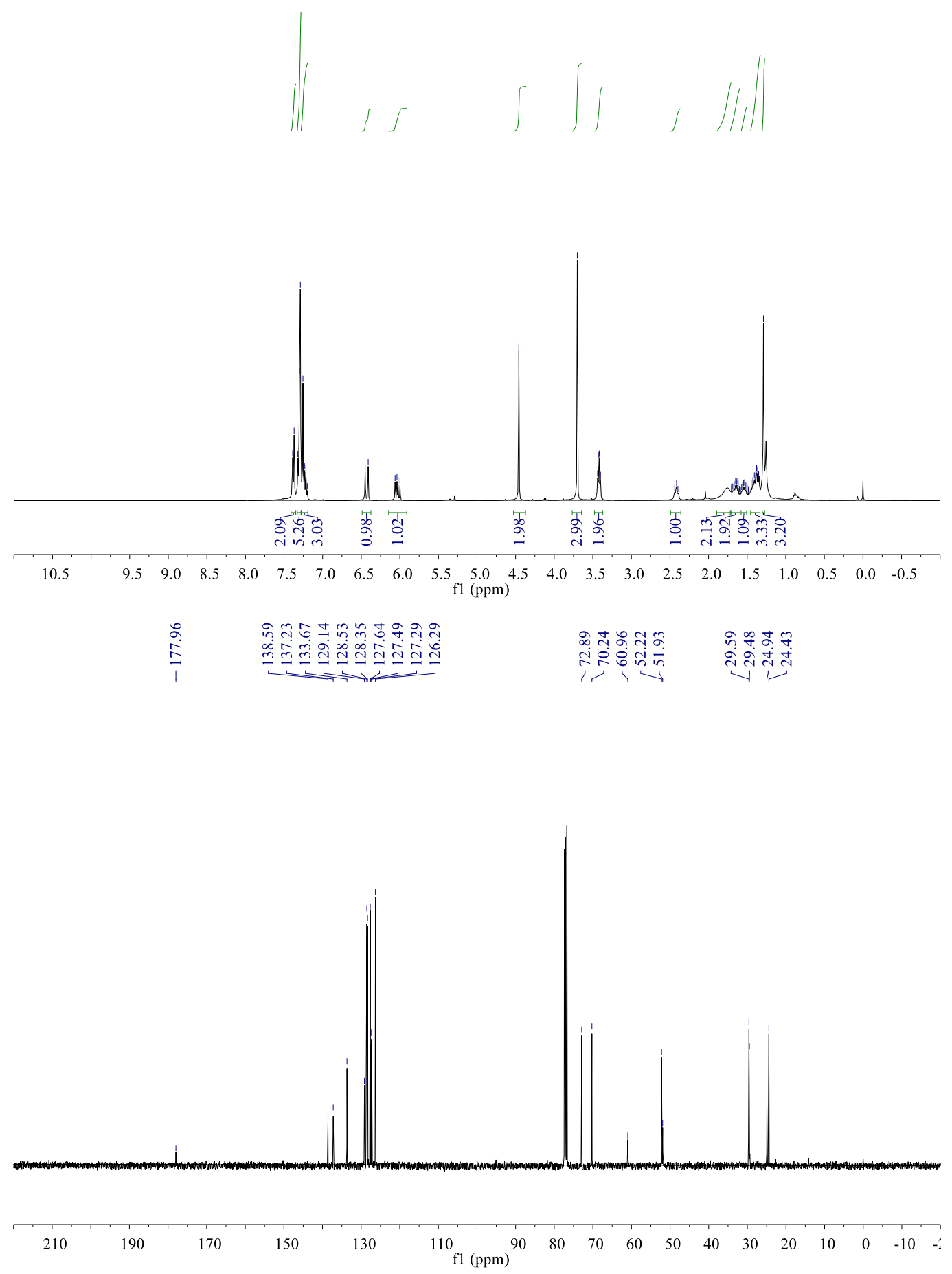


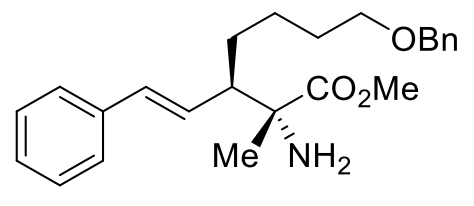

\section{$(2 R, 3 R)-3 q a$}

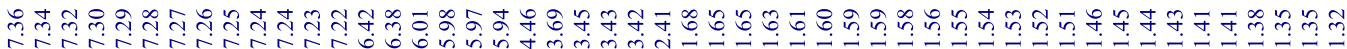
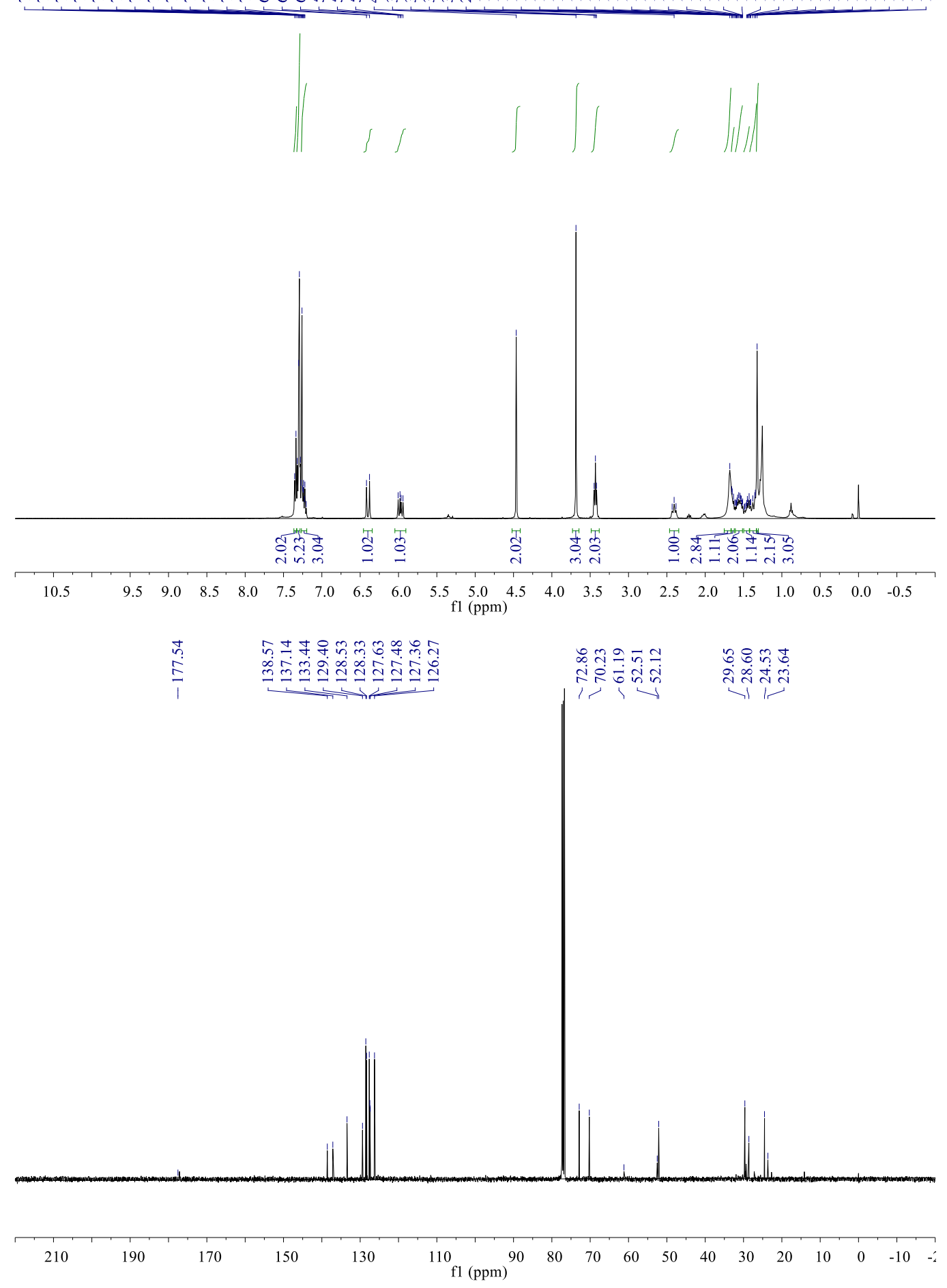
<smiles>CCC(N)(C(C)=O)C(C)/C=C/c1ccccc1</smiles>

$(2 S, 3 R)-3 a b$

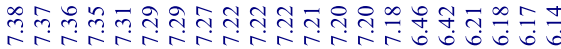

I

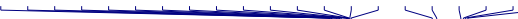

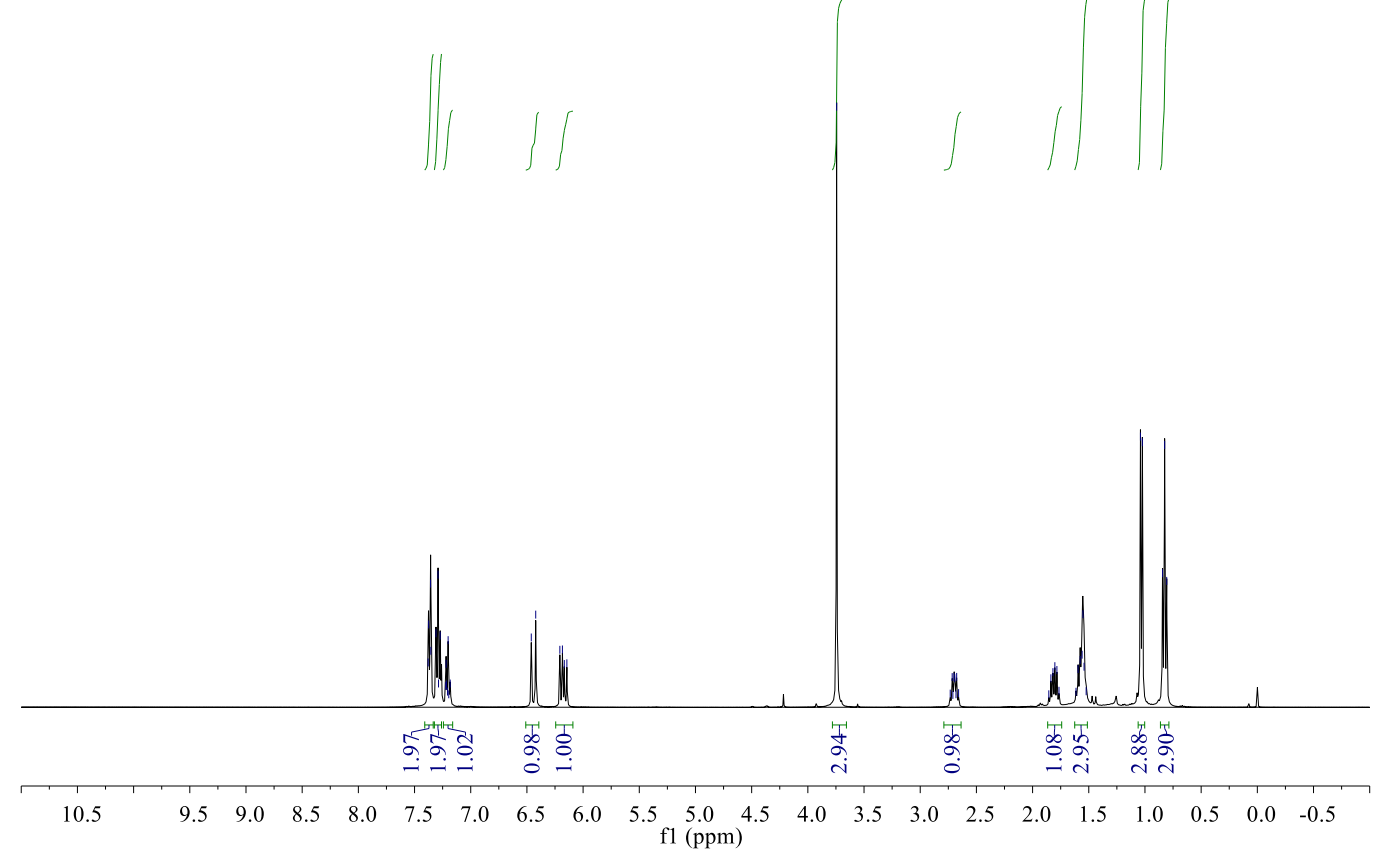
U

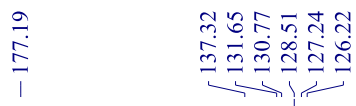

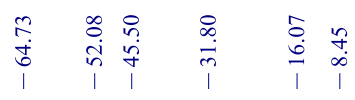
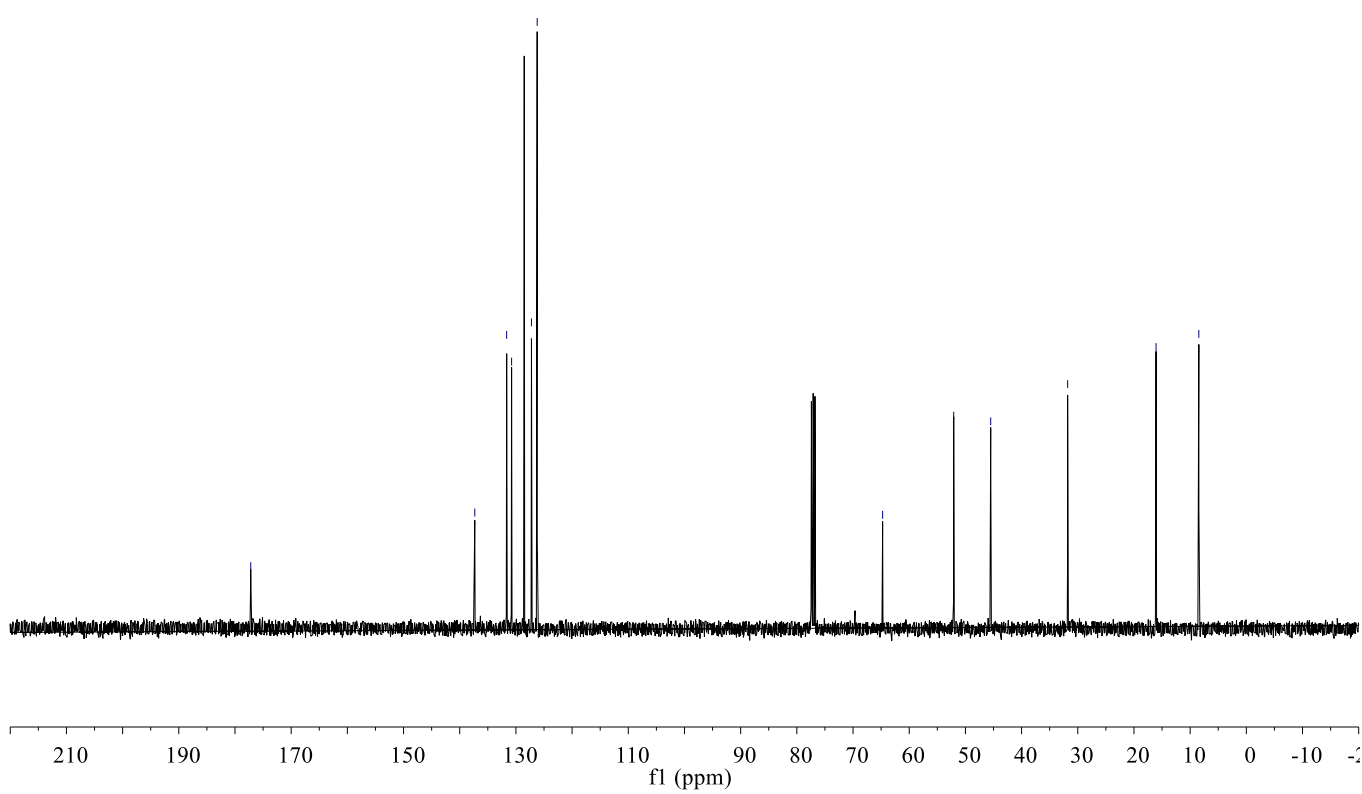
<smiles>CCC(N)(C(C)=O)C(C)/C=C/c1ccccc1</smiles>

$(2 R, 3 R)-3 \mathbf{a b}$

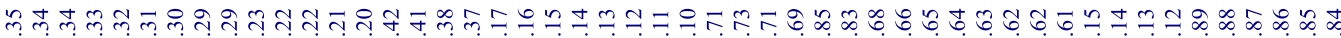

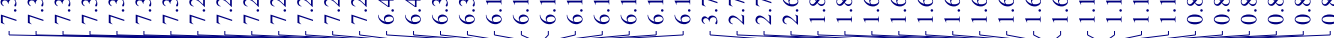
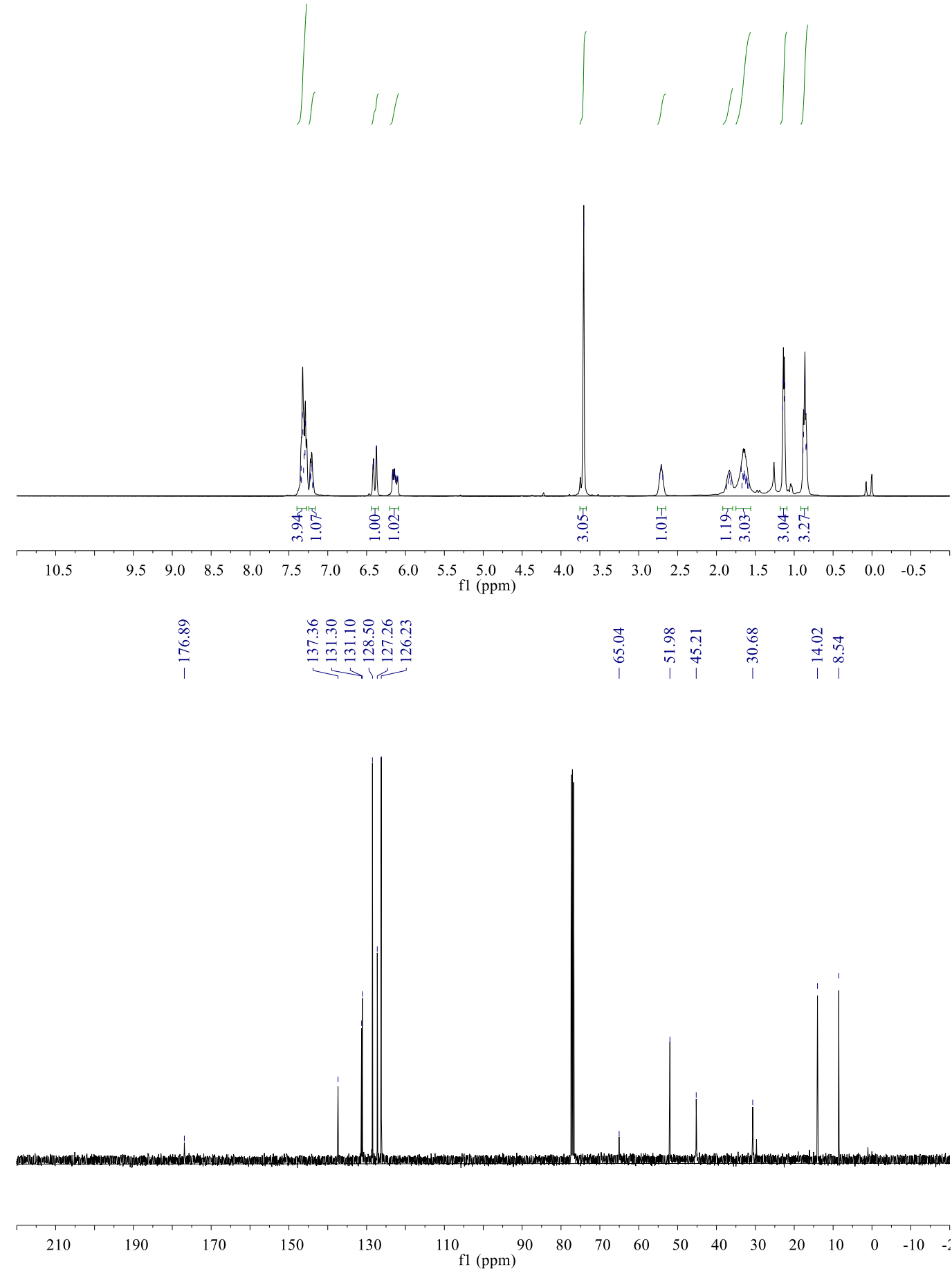
<smiles>COC(=O)[C@](N)(C(C)C)C(C)/C=C/c1ccccc1</smiles>

$(2 S, 3 R)-3 a c$

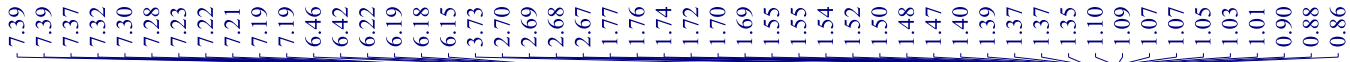
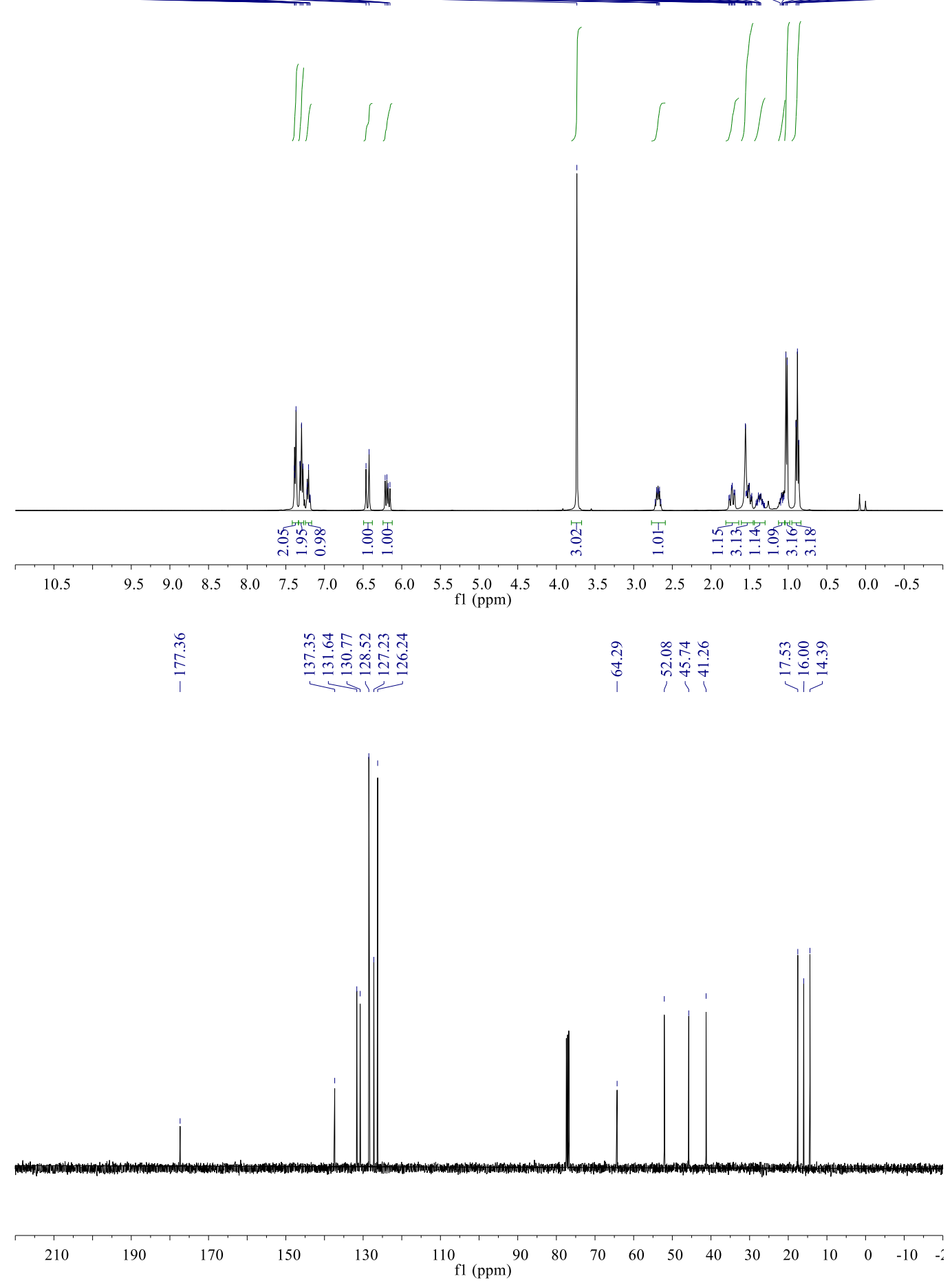


$$
\overbrace{(2 S, 3 R)-3 \mathbf{a d}}^{\mathrm{Me}} \overbrace{\mathrm{H}_{2} \mathrm{~N}-\mathrm{Bu}}^{\mathrm{CO}_{2} \mathrm{Me}}
$$

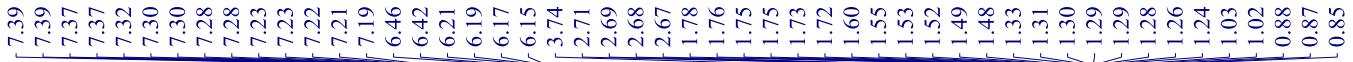
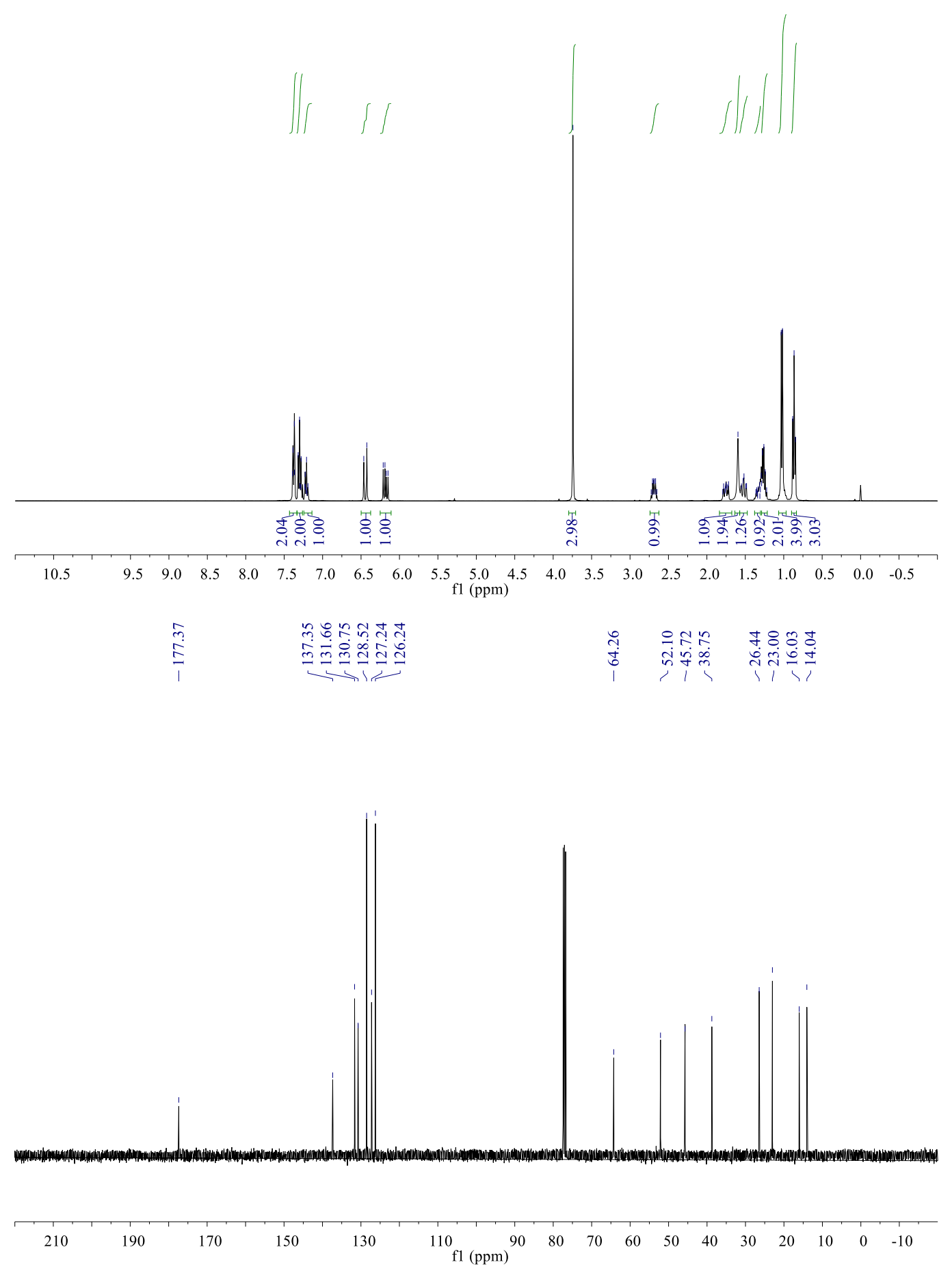
<smiles>COC(=O)[C@](N)(CCc1ccccc1)[C@H](C)/C=C/c1ccccc1</smiles>

$(2 S, 3 R)-3 \mathbf{a e}$

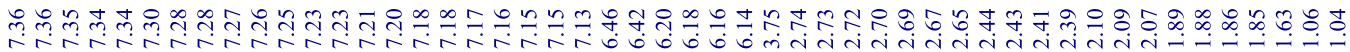

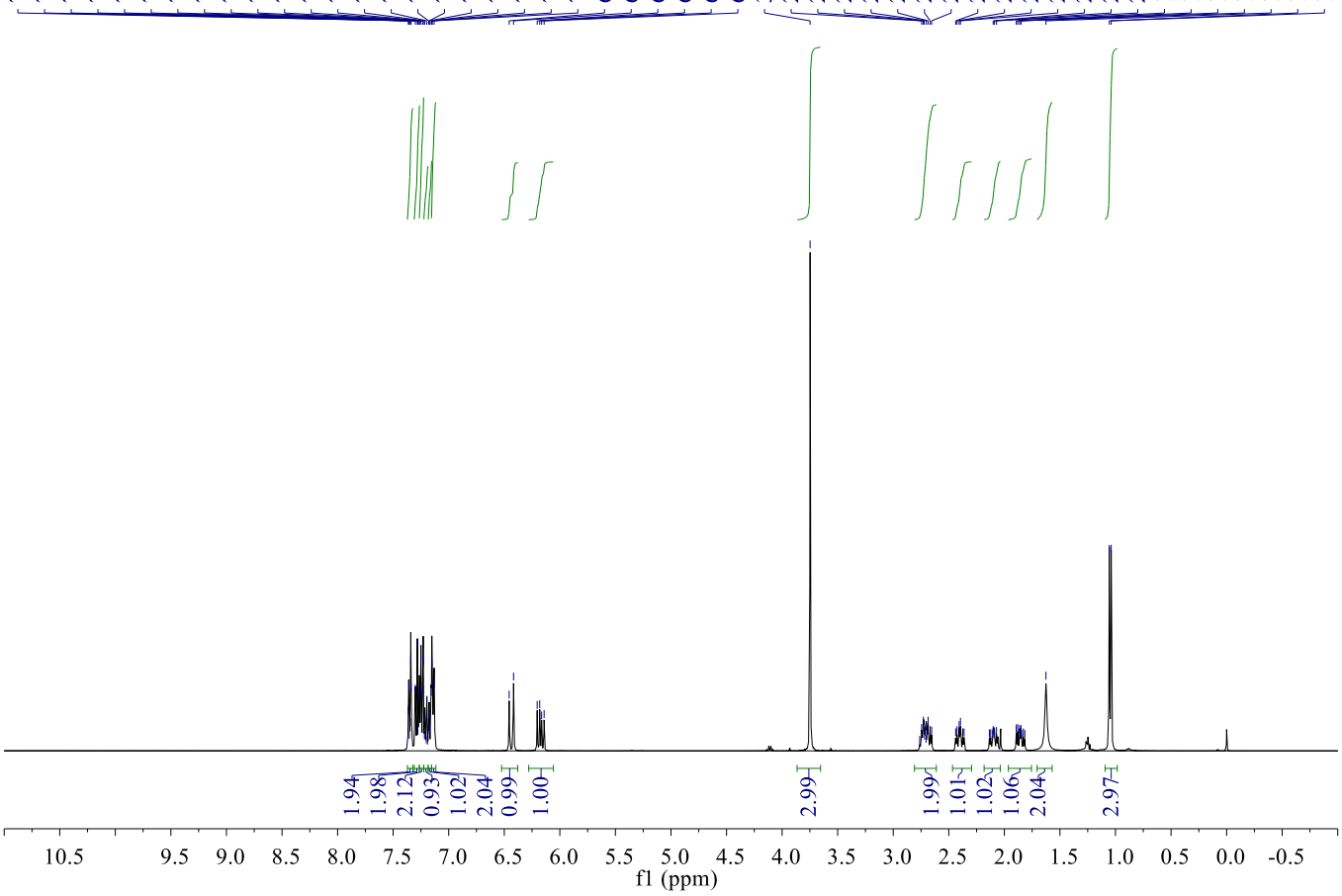

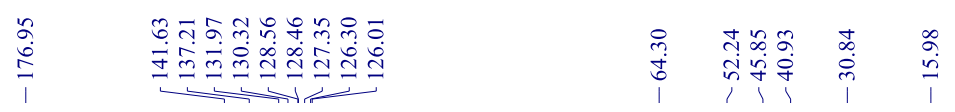
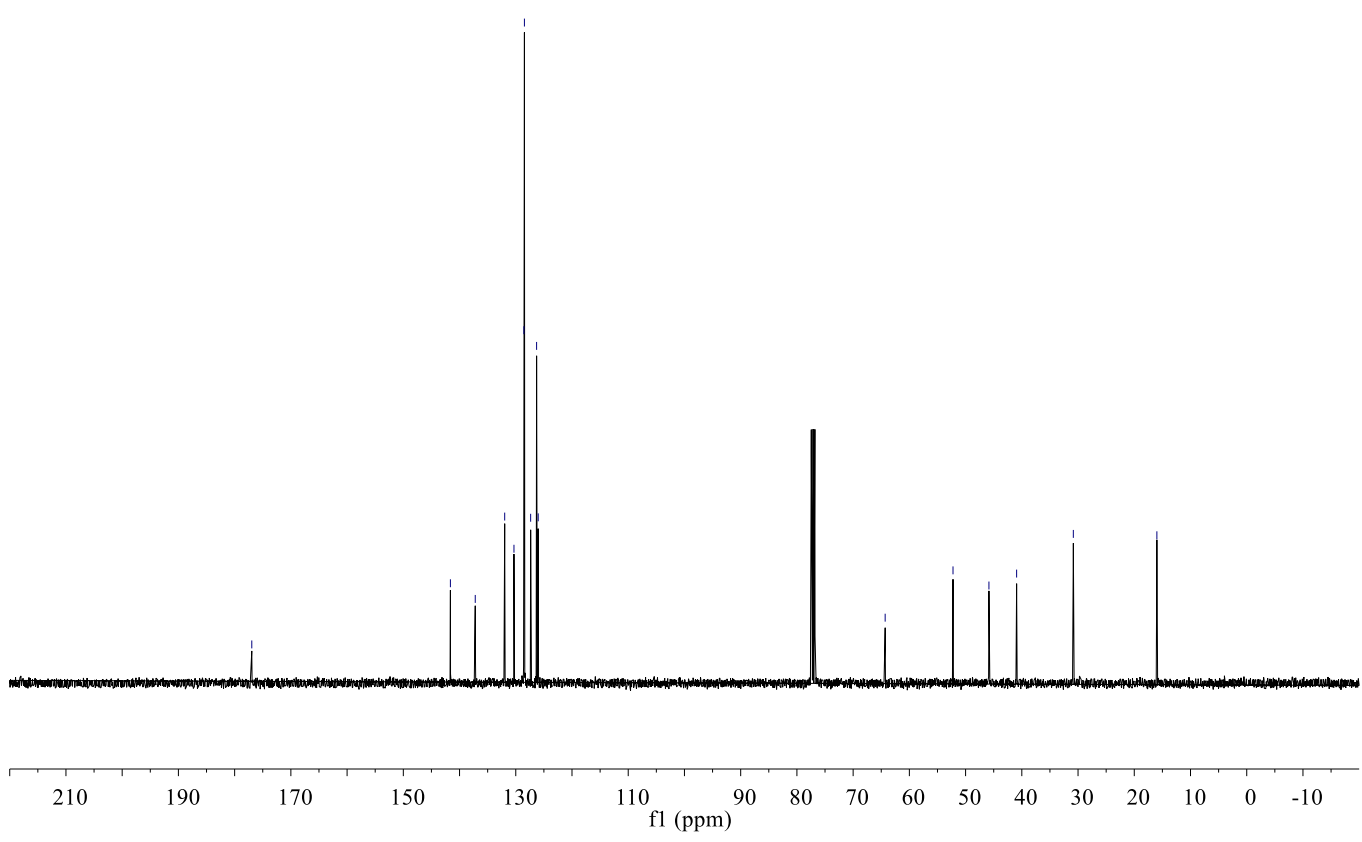

S107 


$$
\overbrace{(2 S, 3 R)-3 \text { af }}^{M e}
$$

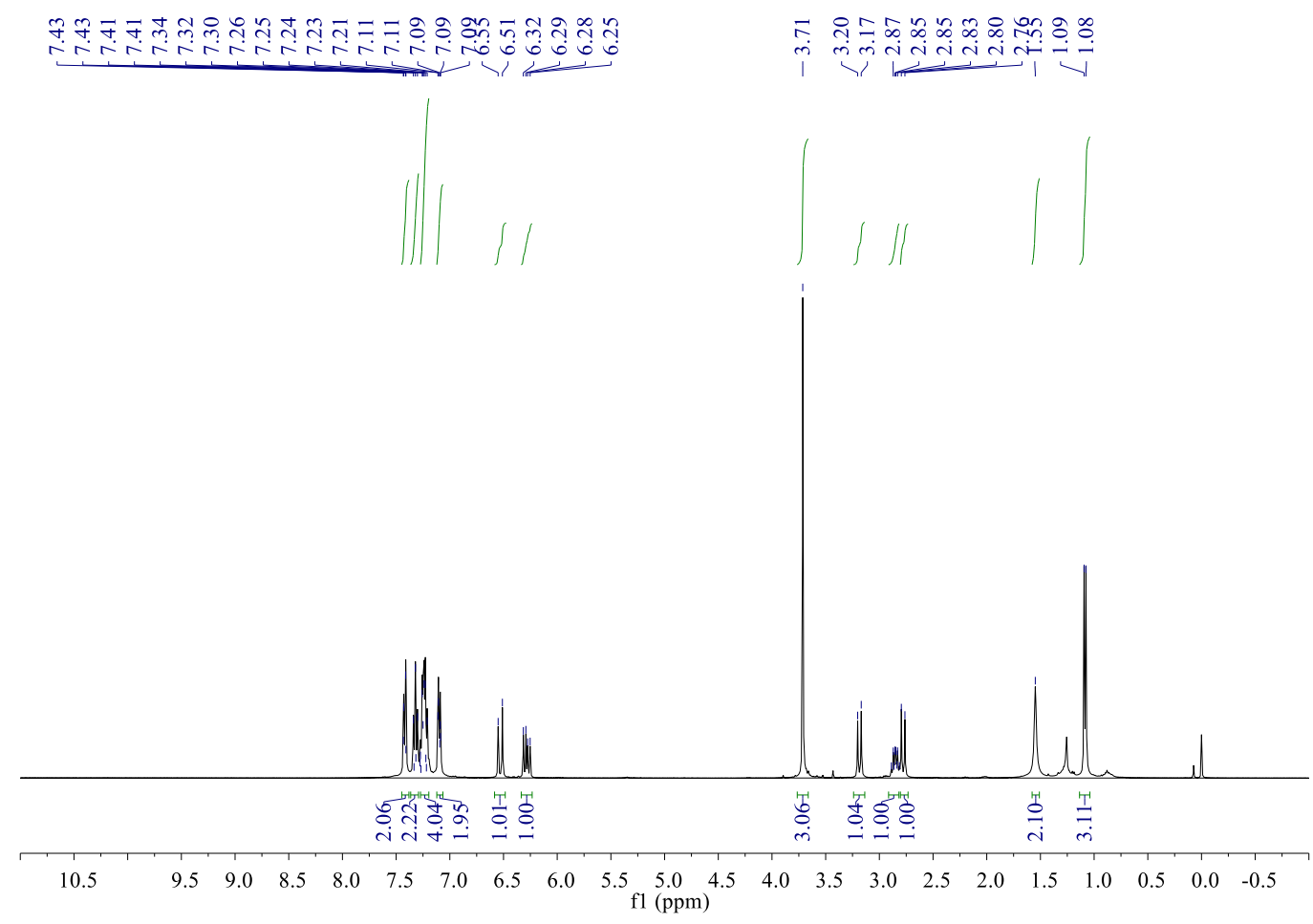

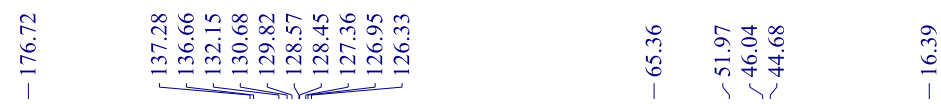
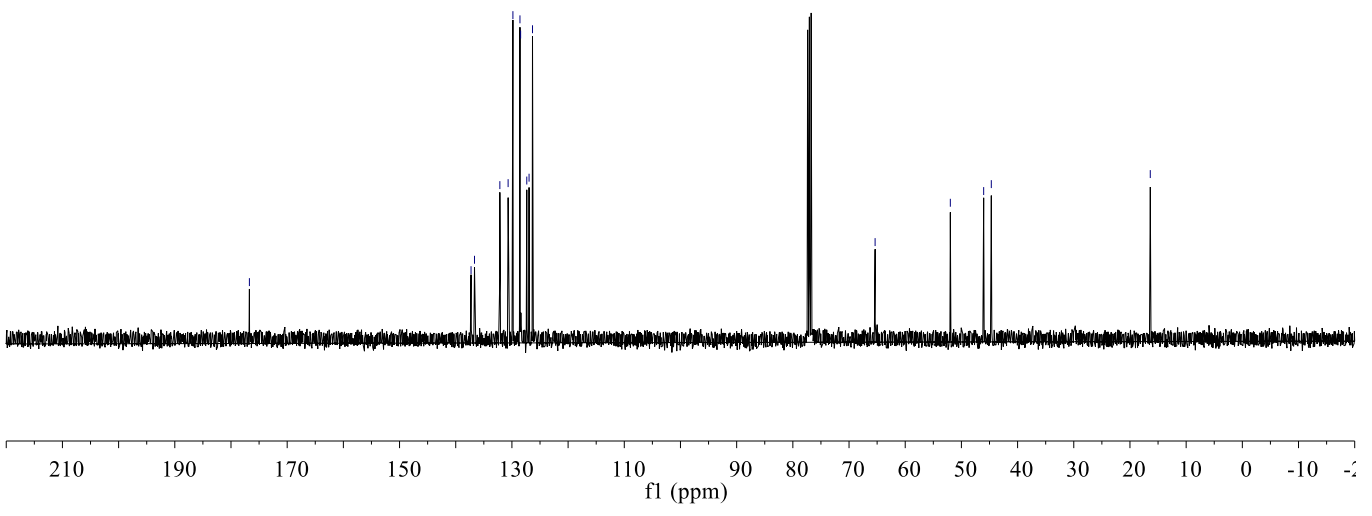


$$
\overbrace{\mathrm{H}_{2} \mathrm{~N}}^{\mathrm{Me}} \mathrm{CO}_{2 \mathrm{CO}} \mathrm{Me}
$$

(2S,3R)-3ag
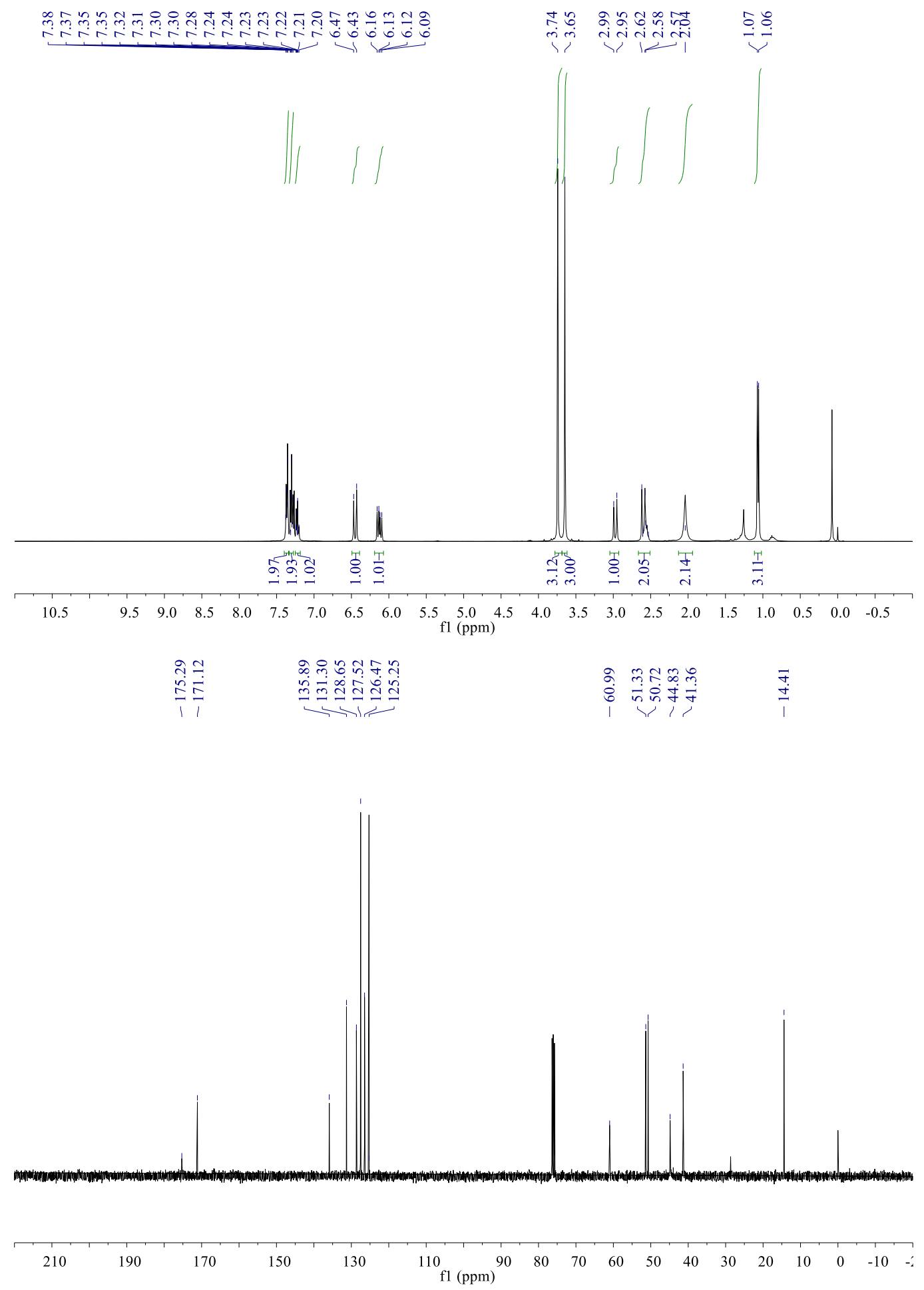


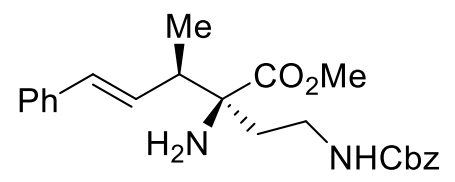

(2S,3R)-3ah
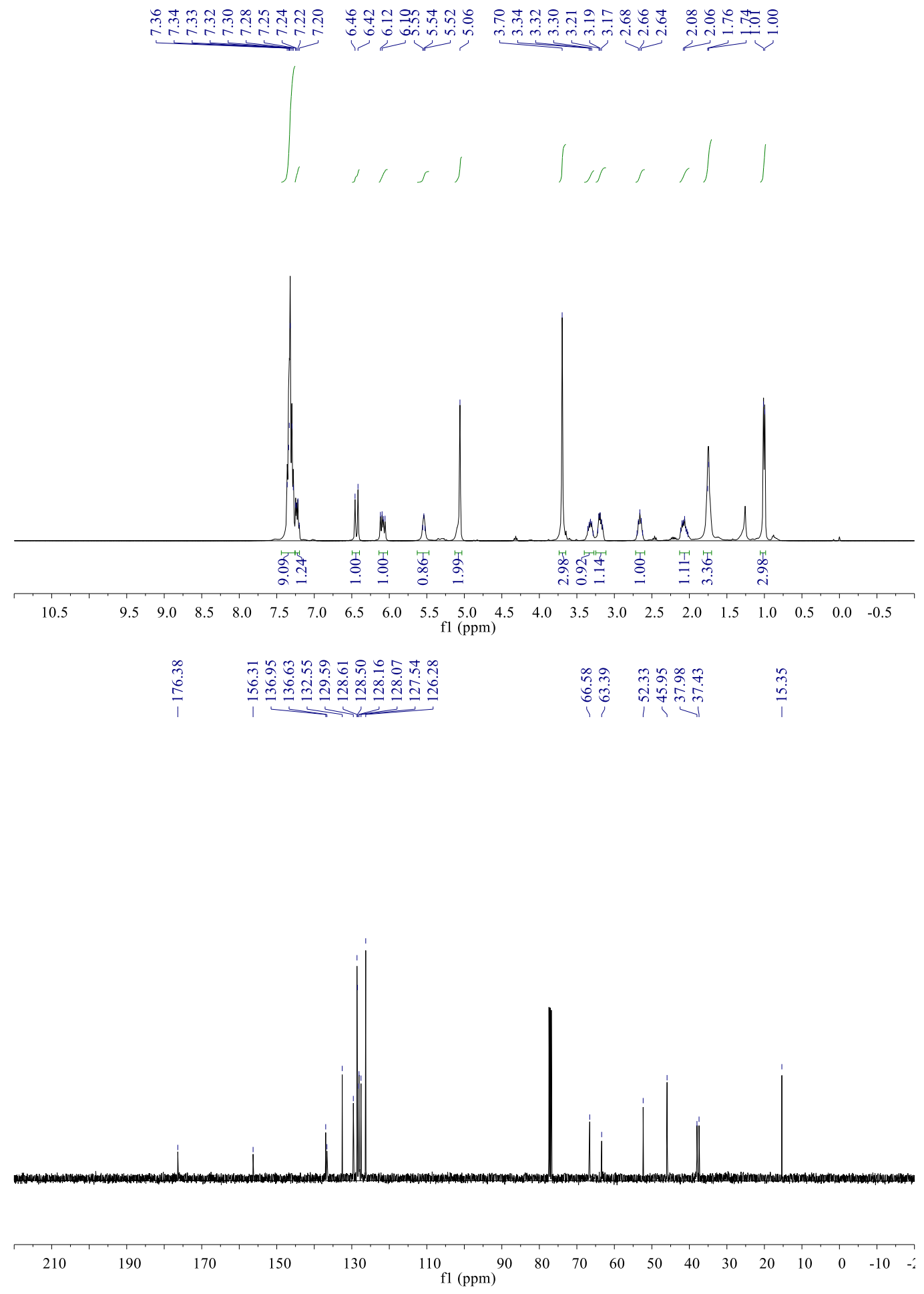
<smiles>COC(=O)[C@](N)(/C=C/c1ccccc1)[C@@H](C)/C=C/[Mg]</smiles>

象央 每
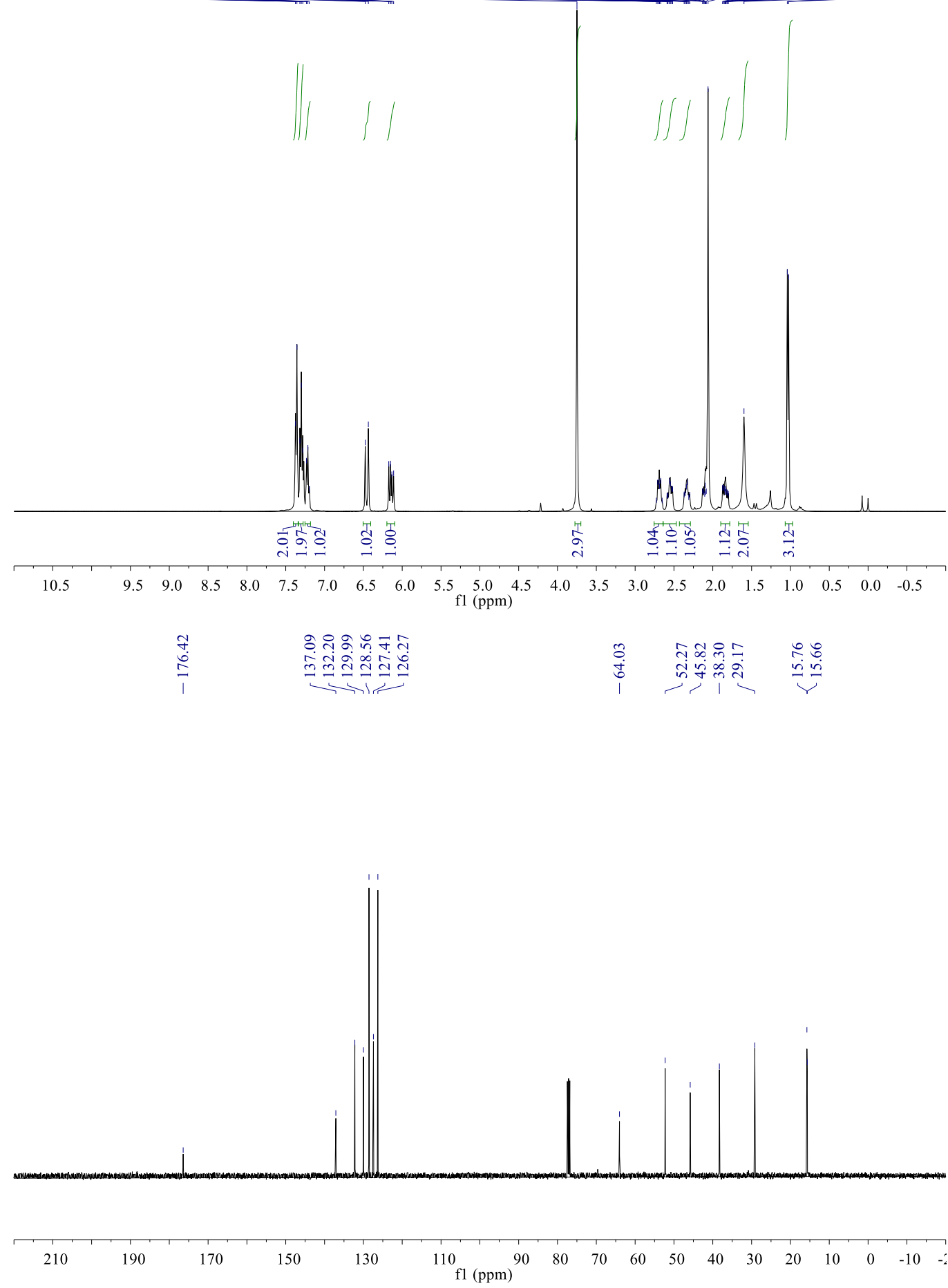
<smiles>C=CC[C@](N)(C(=O)OC)[C@@H](C)/C=C/c1ccccc1</smiles>

$(2 S, 3 R)-3$ aj

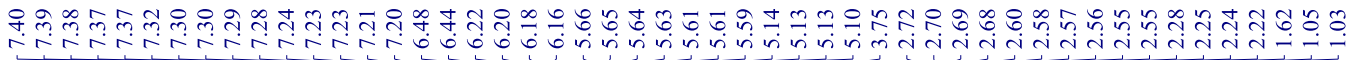

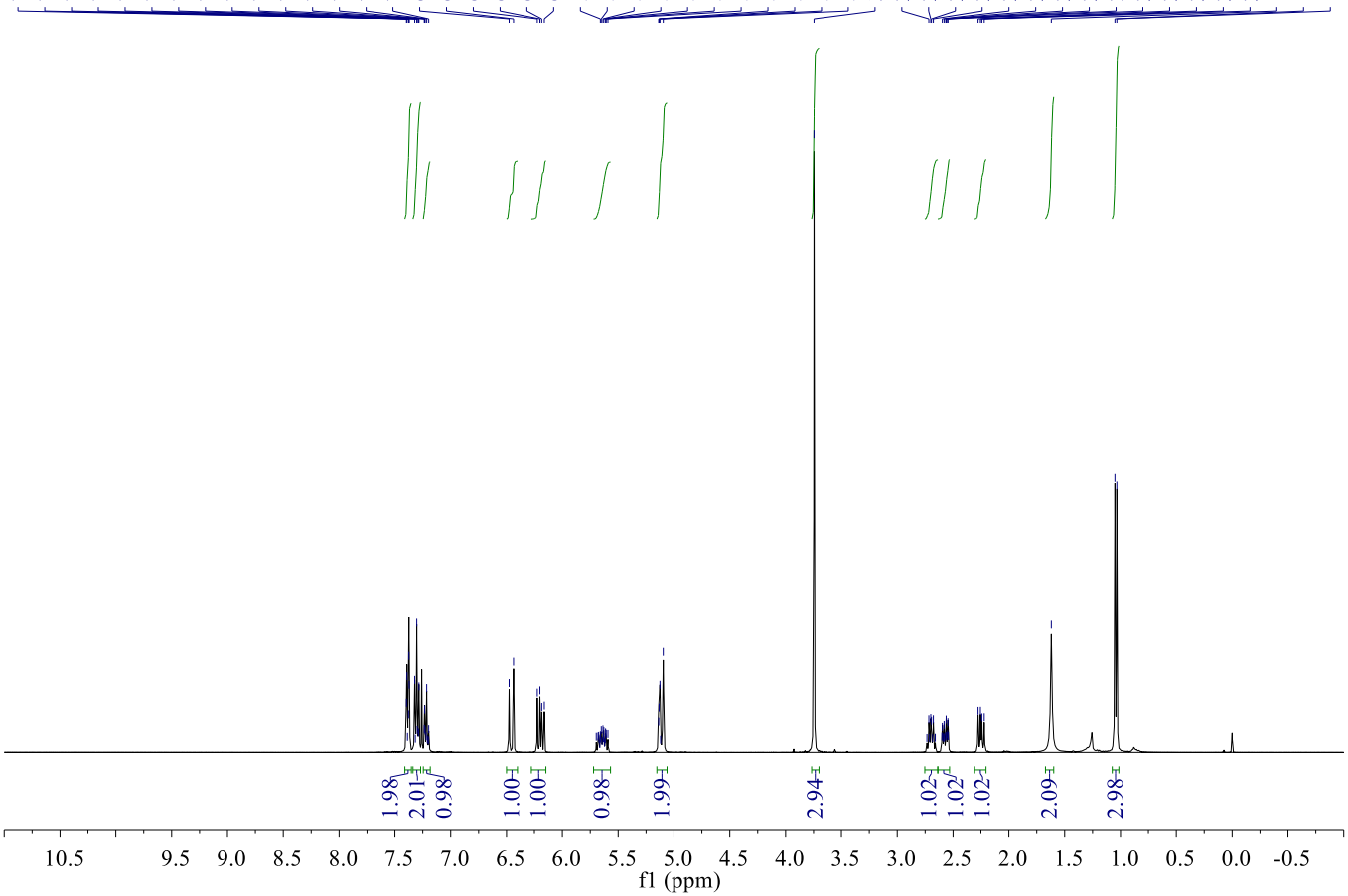

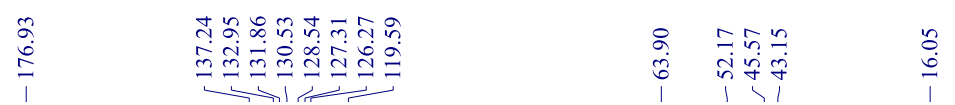

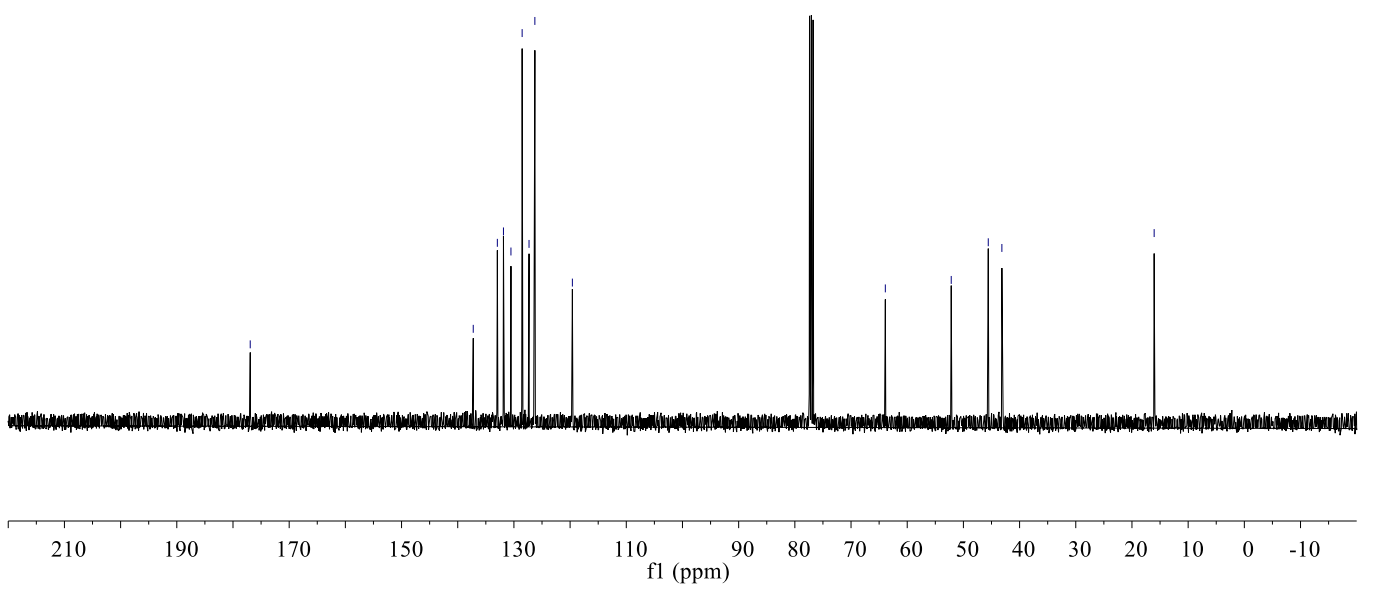



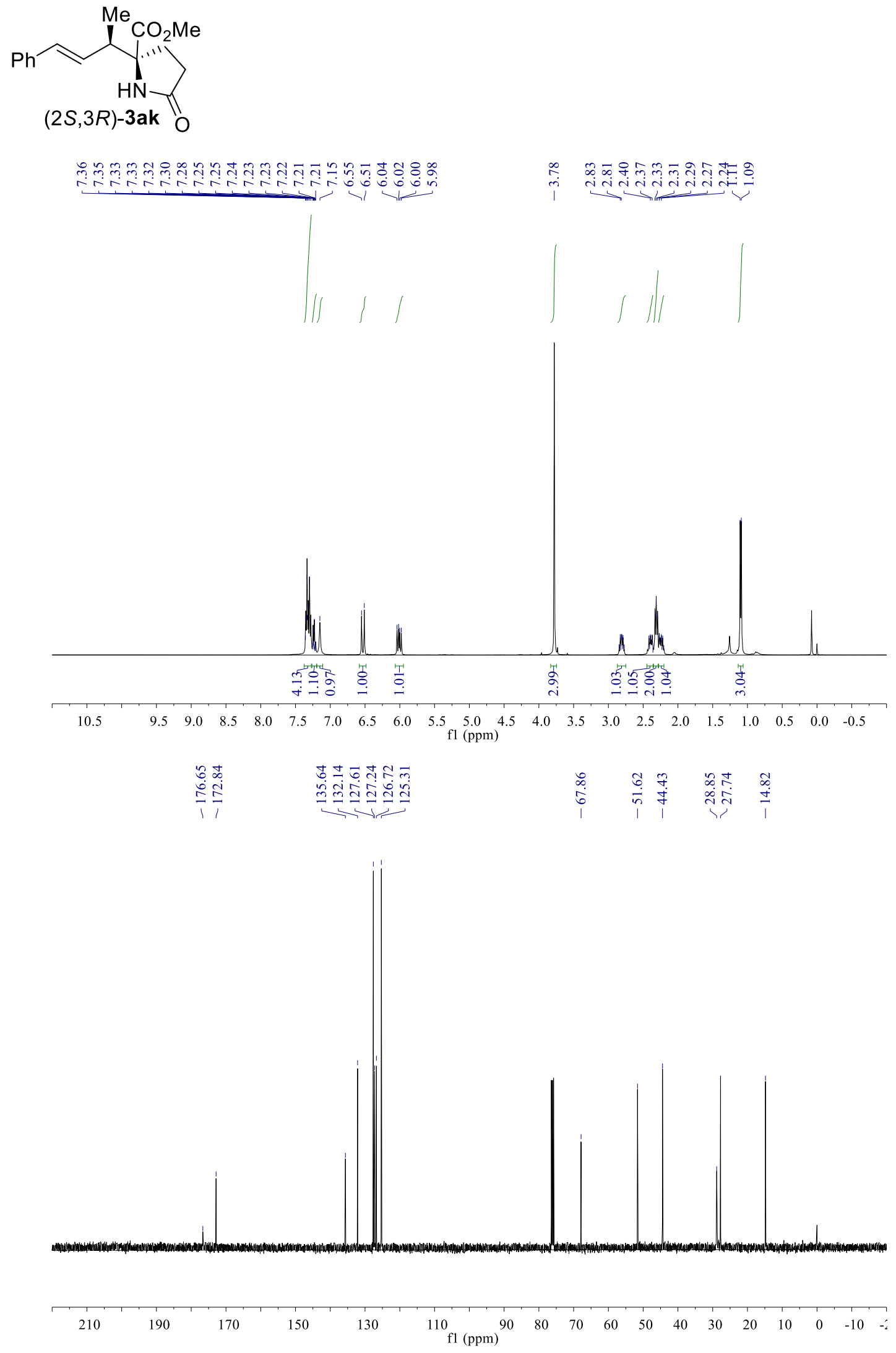
<smiles>[Y19]C(/C=C/c1ccccc1)[C@@]1(N)CCOC1=O</smiles>

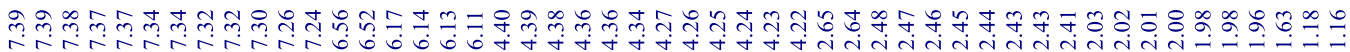
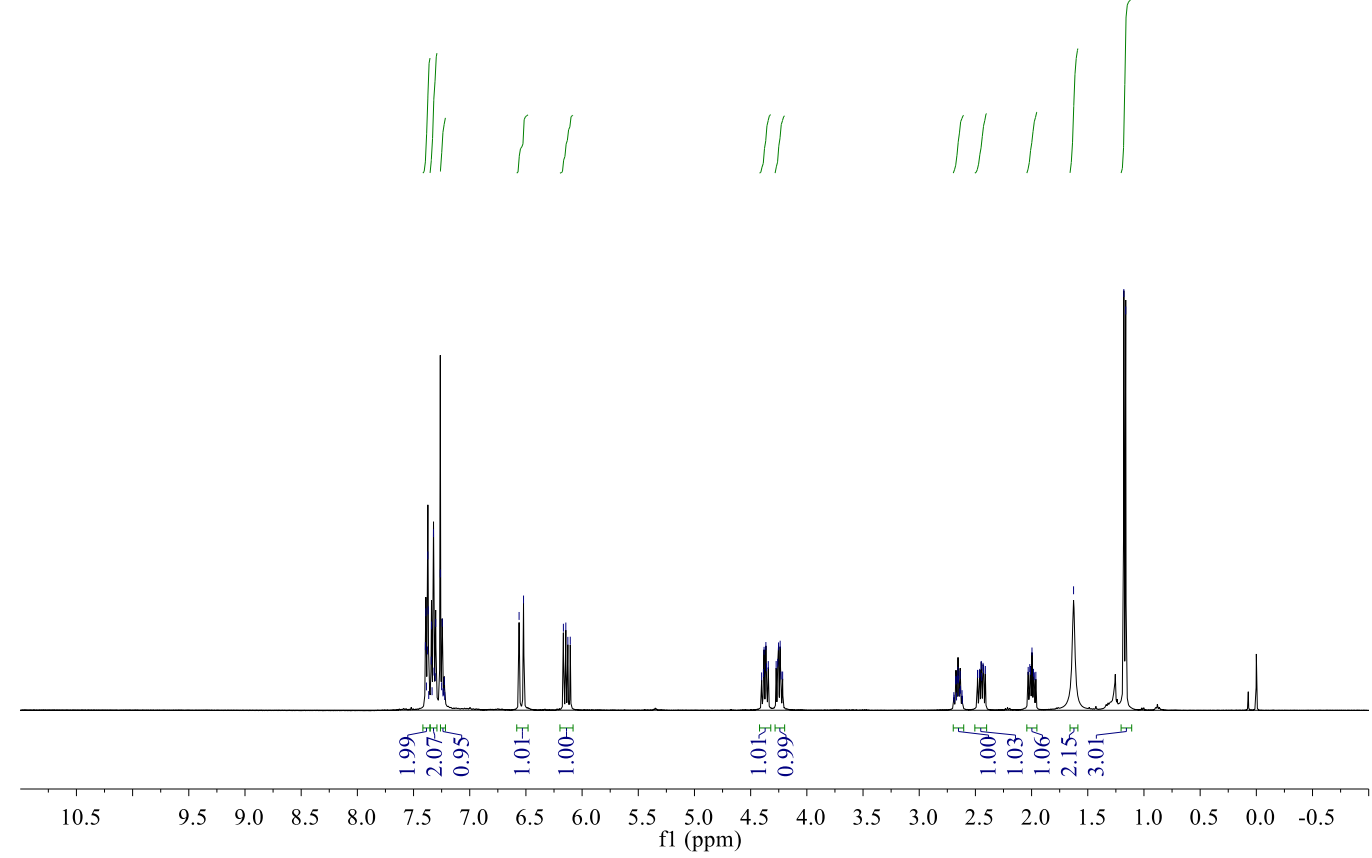

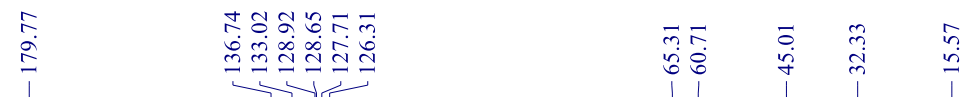

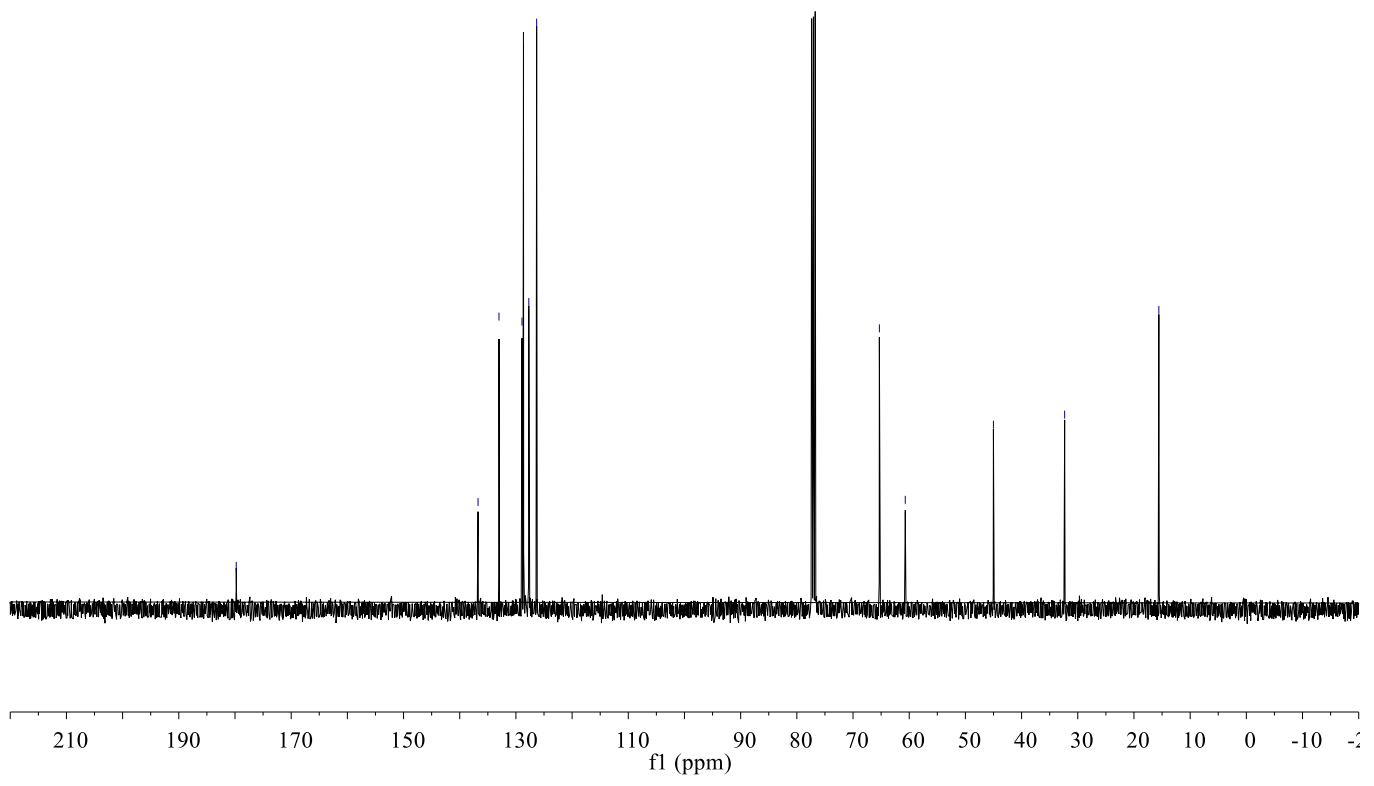


<smiles>CC(=O)C1(C(C)=O)N=C(C)C[C@H]1C=Cc1ccccc1</smiles>

(2S,3R)-3am $\mathrm{Ph}$

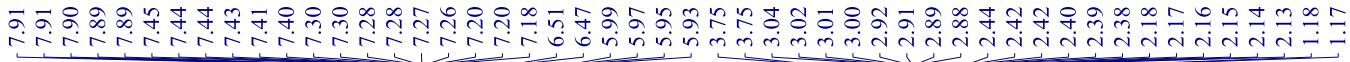

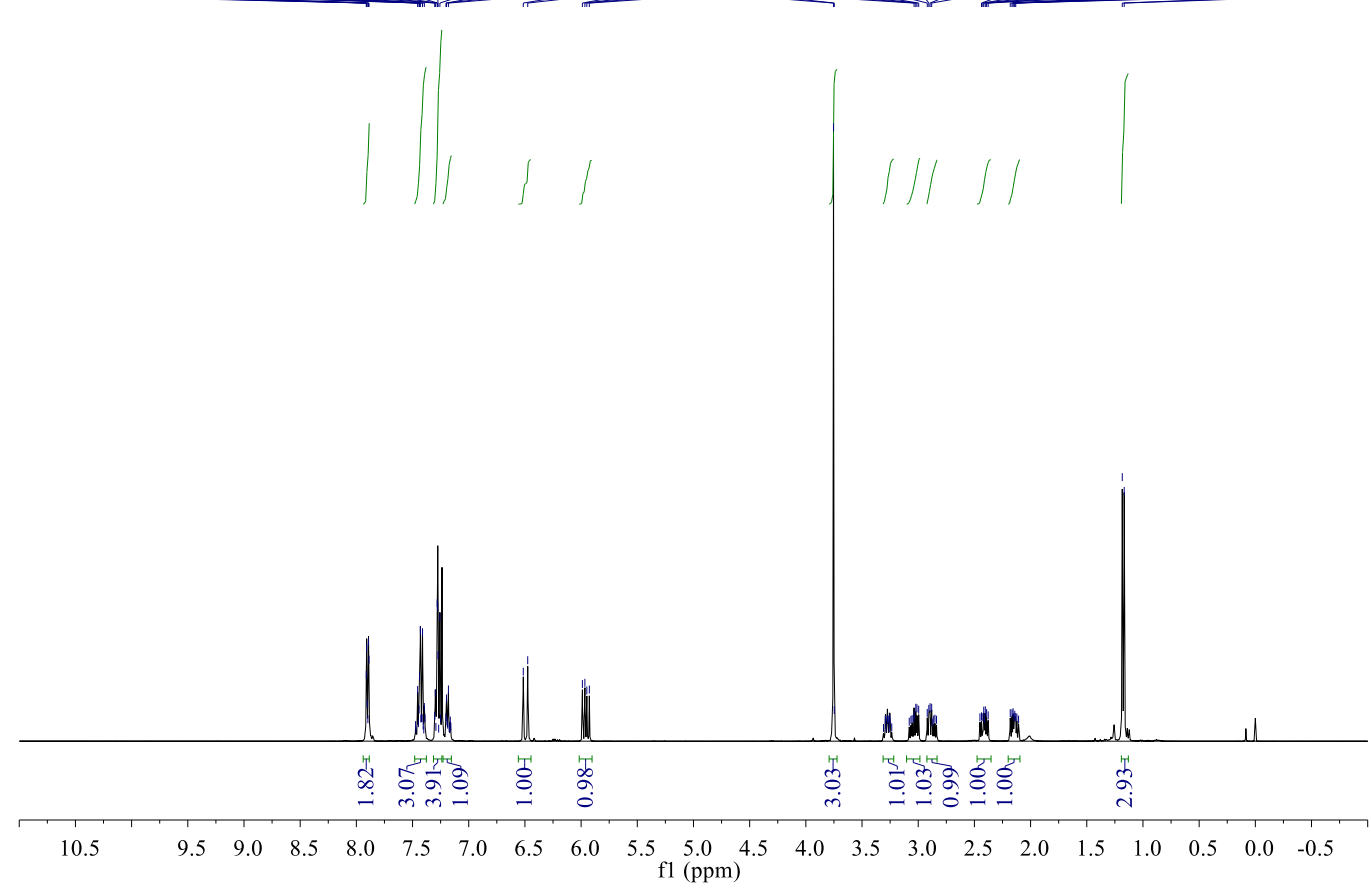

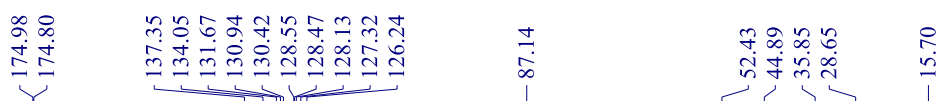
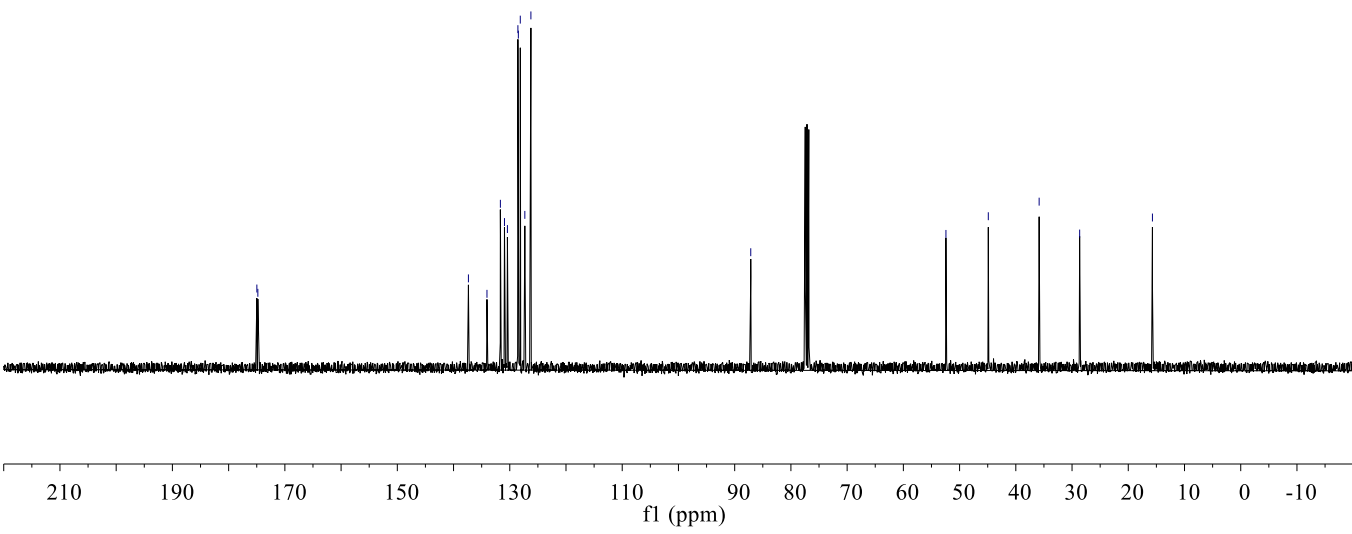
$\overbrace{\mathrm{H}_{2} \mathrm{~N}}^{\mathrm{Me}} \mathrm{CO}_{2} \mathrm{Et}$

$(2 S, 3 R)-3$ an

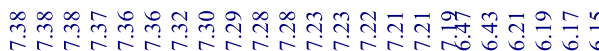

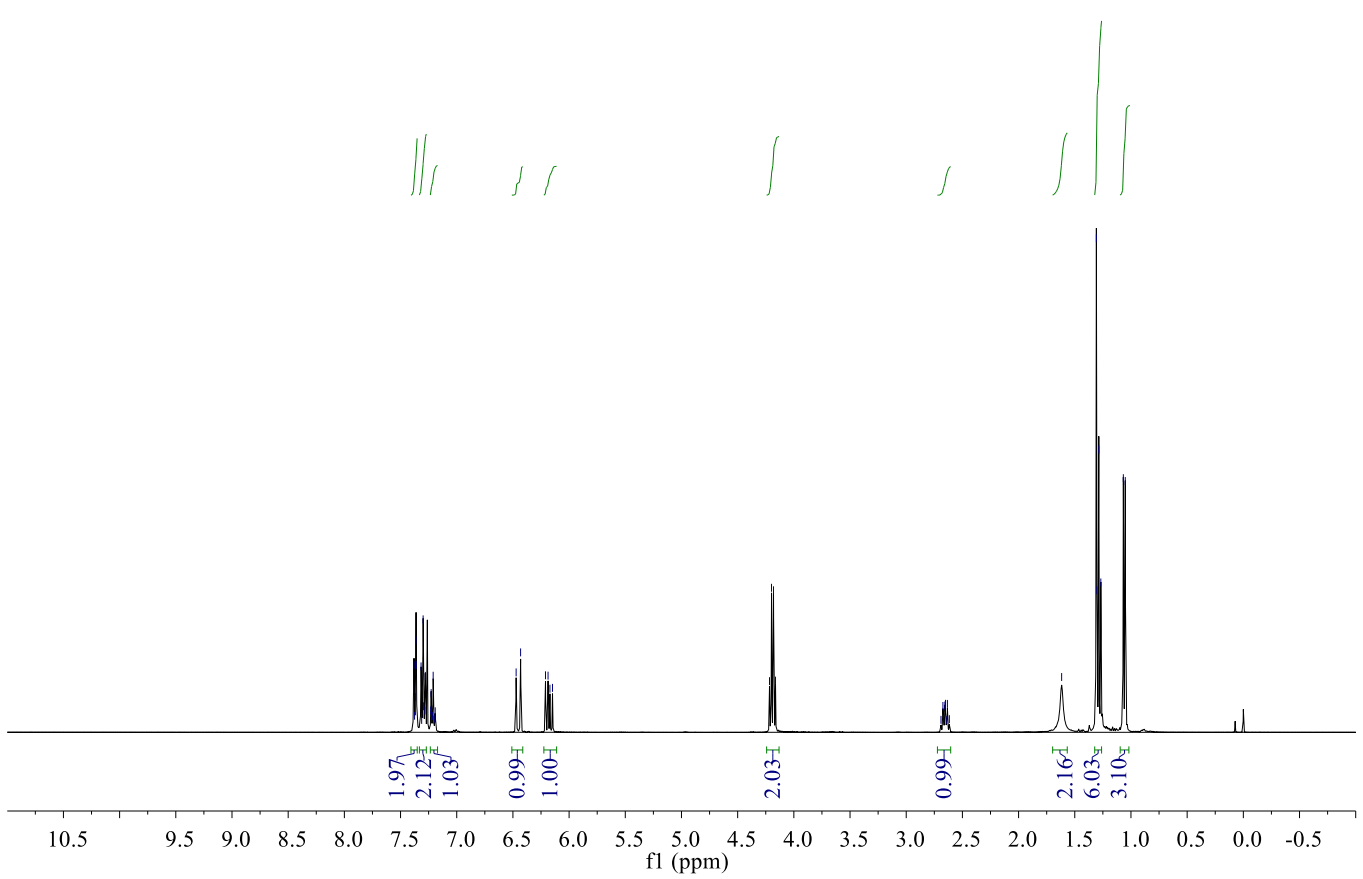

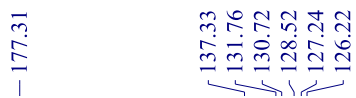

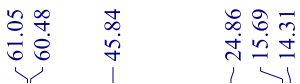

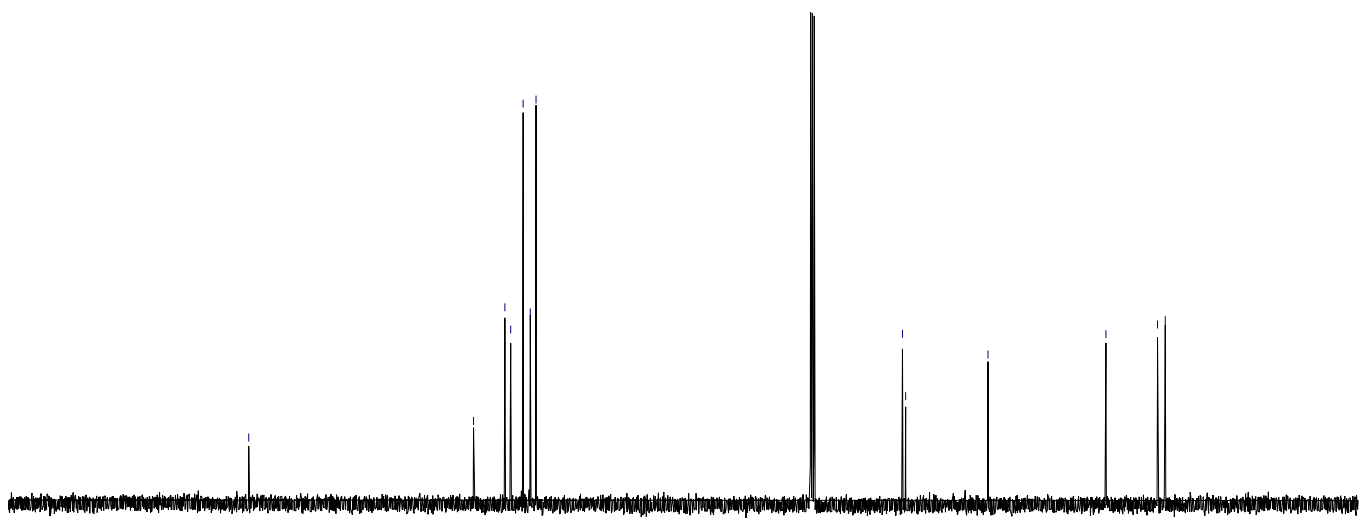

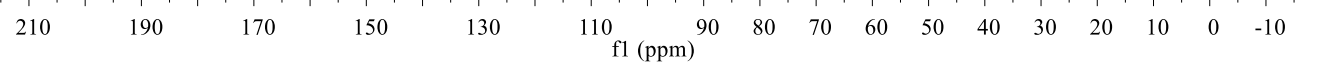

S116 
<smiles>CCCCCC(C)C(N)C(C)C(=O)OCCC</smiles>

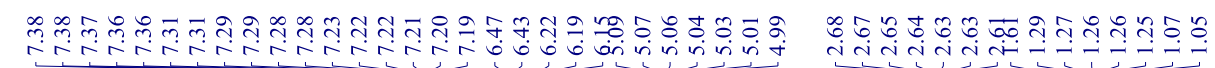
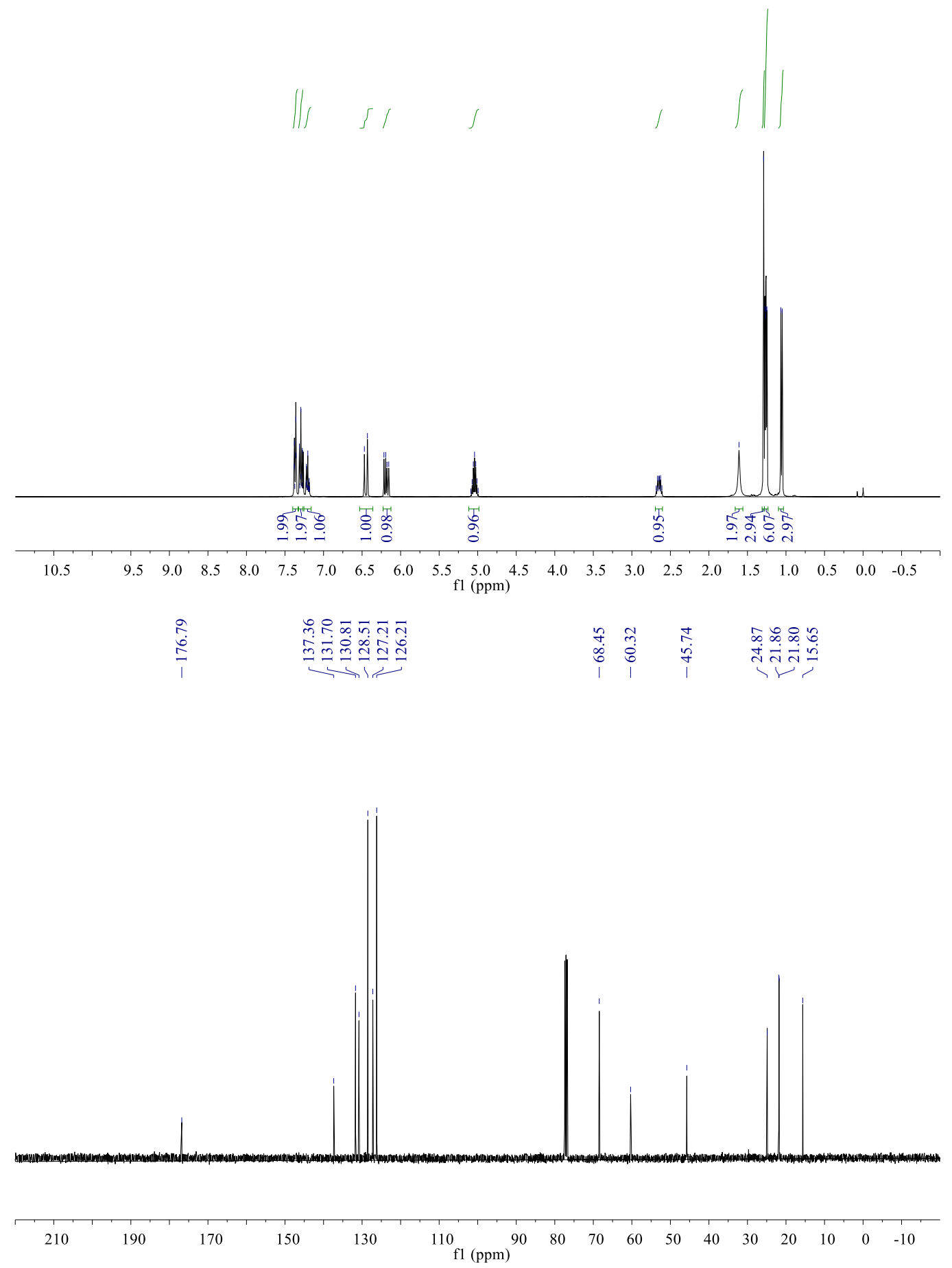


$$
\overbrace{(2 S, 3 R)-3 a p}^{M e} C_{M e}^{\mathrm{H}_{2} \mathrm{~N}}
$$

\section{mim

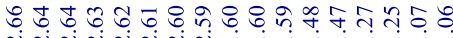

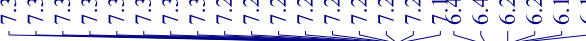
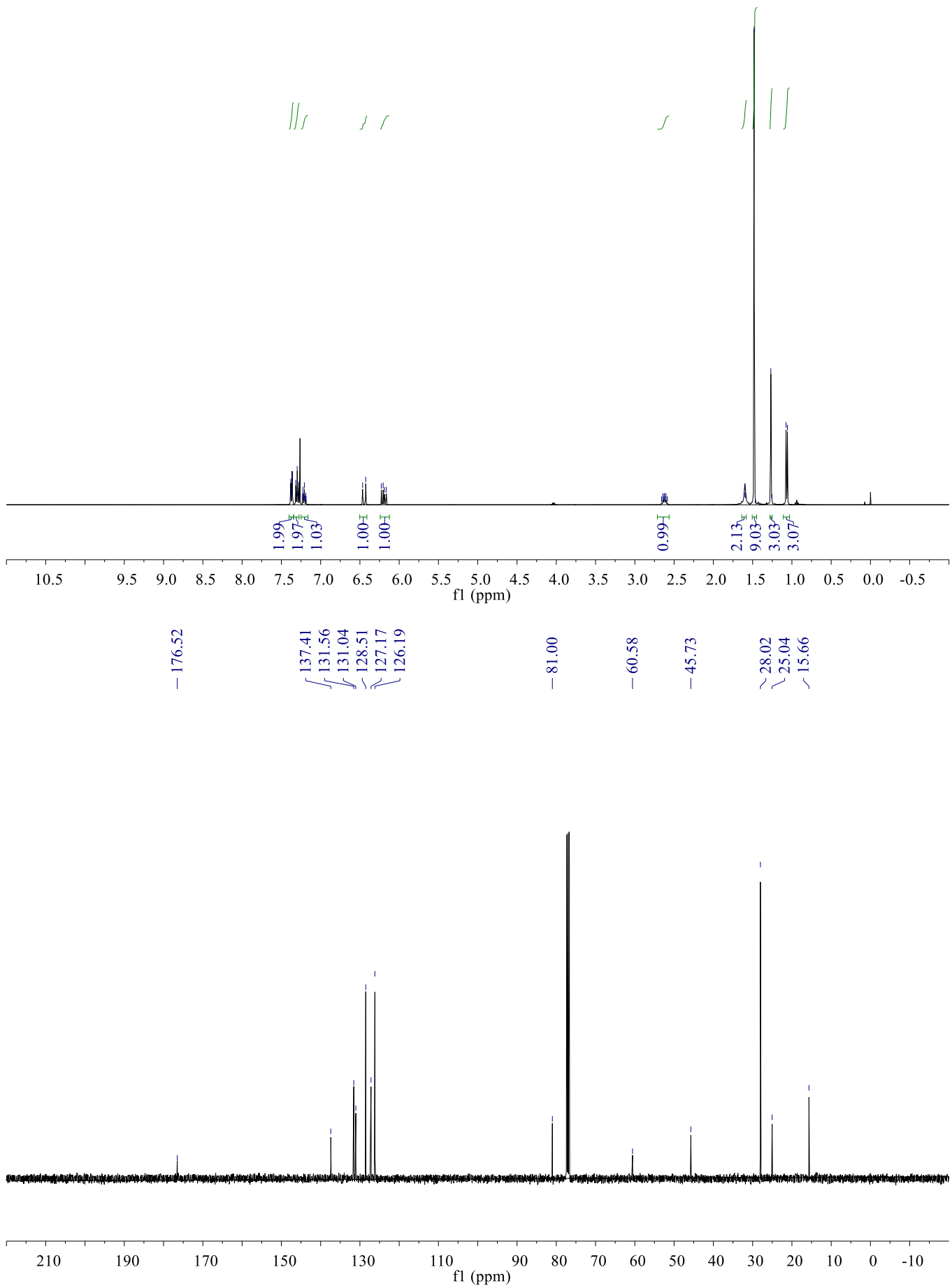
<smiles>CC(=O)C(N=C(c1ccccc1)c1ccccc1)C(C)C=Cc1ccccc1</smiles>

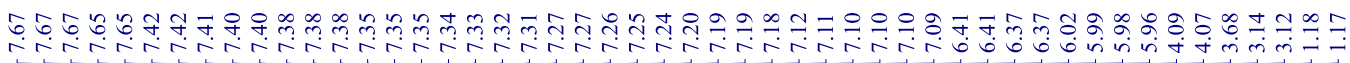
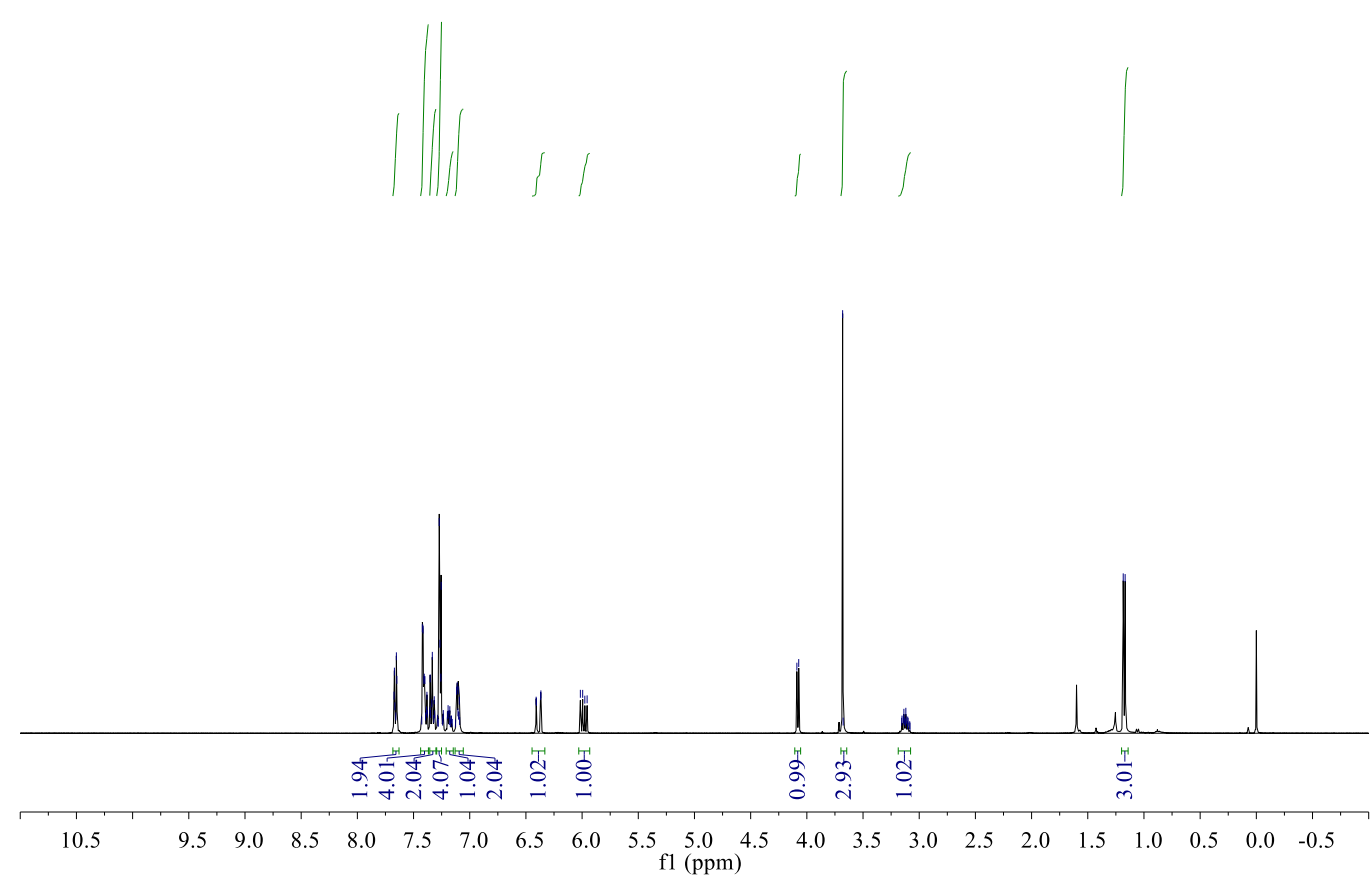

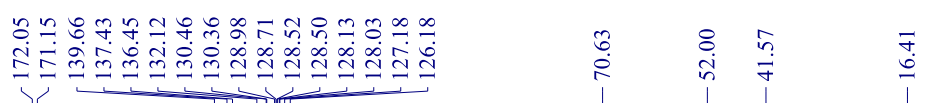

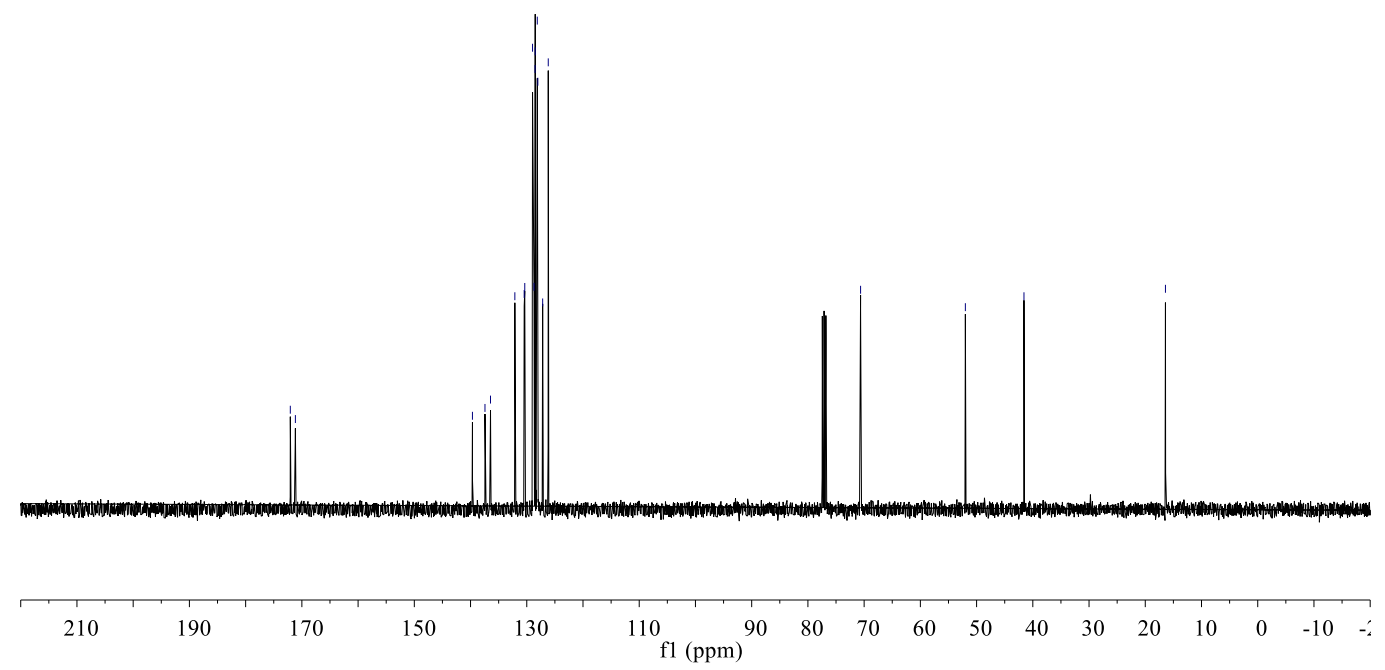


<smiles>CC(=O)C(N=C(c1ccccc1)c1ccccc1)C(C)/C=C/c1ccccc1</smiles>

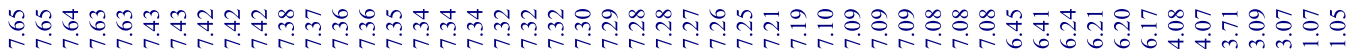

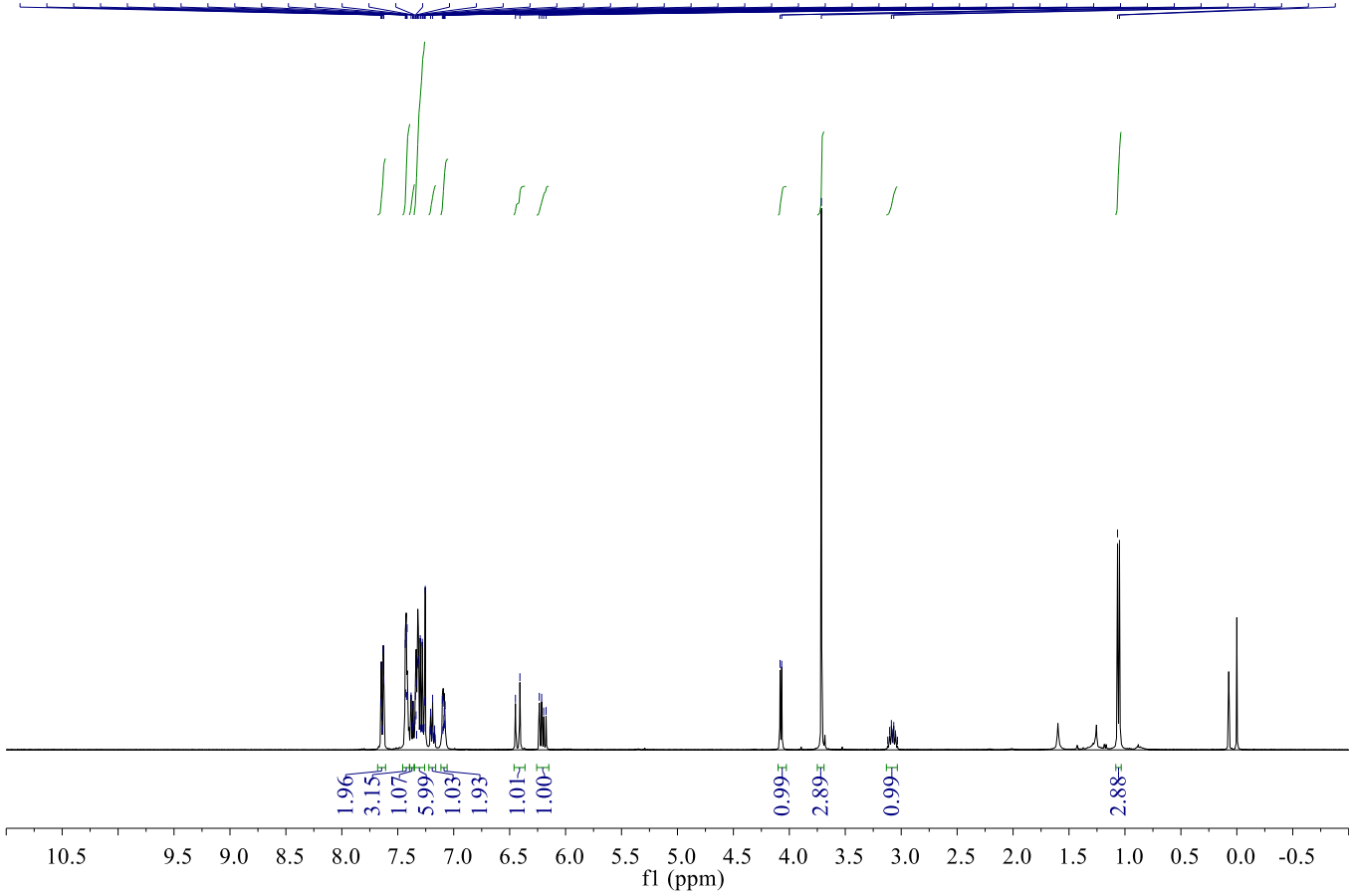

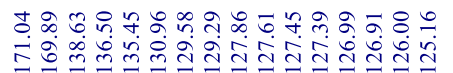

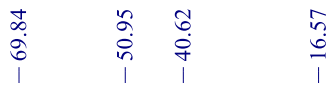

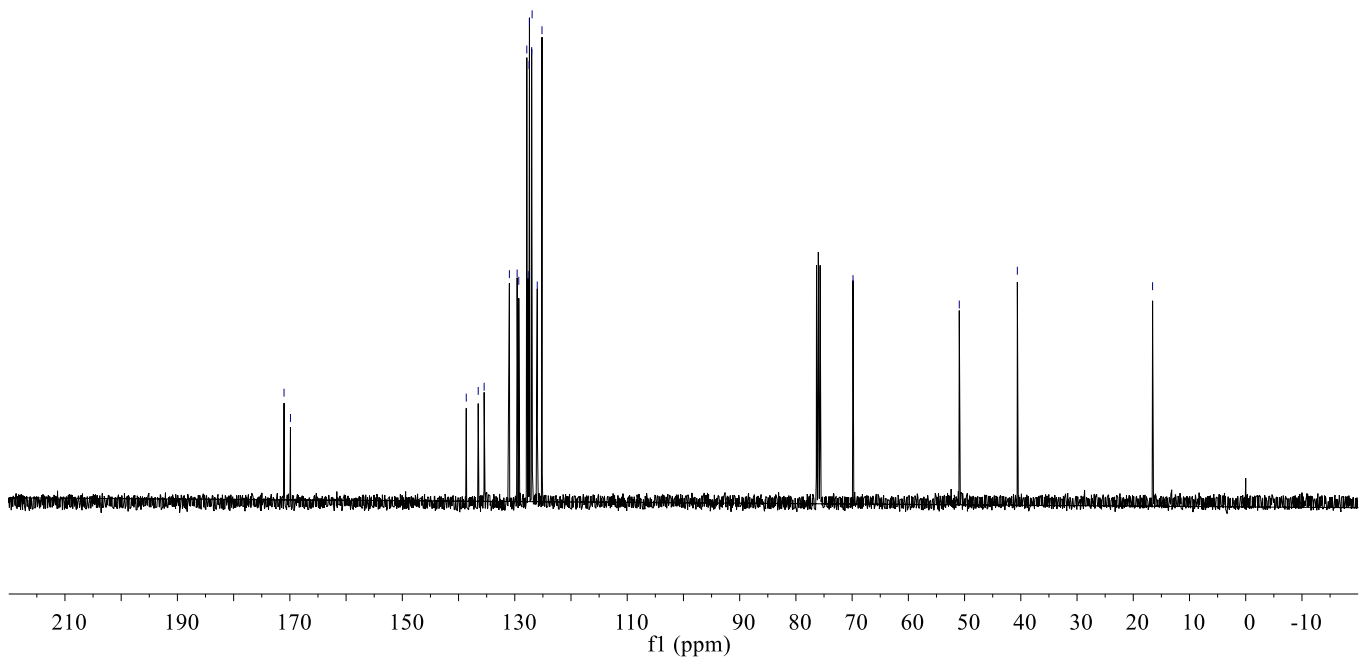


<smiles>C=C/C=C/C(C)C(N=C(c1ccccc1)c1ccccc1)C(C)=O</smiles>

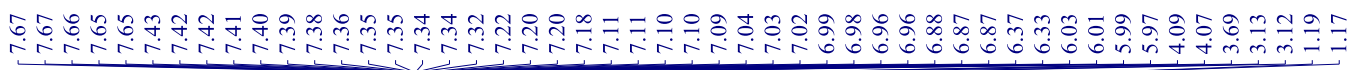

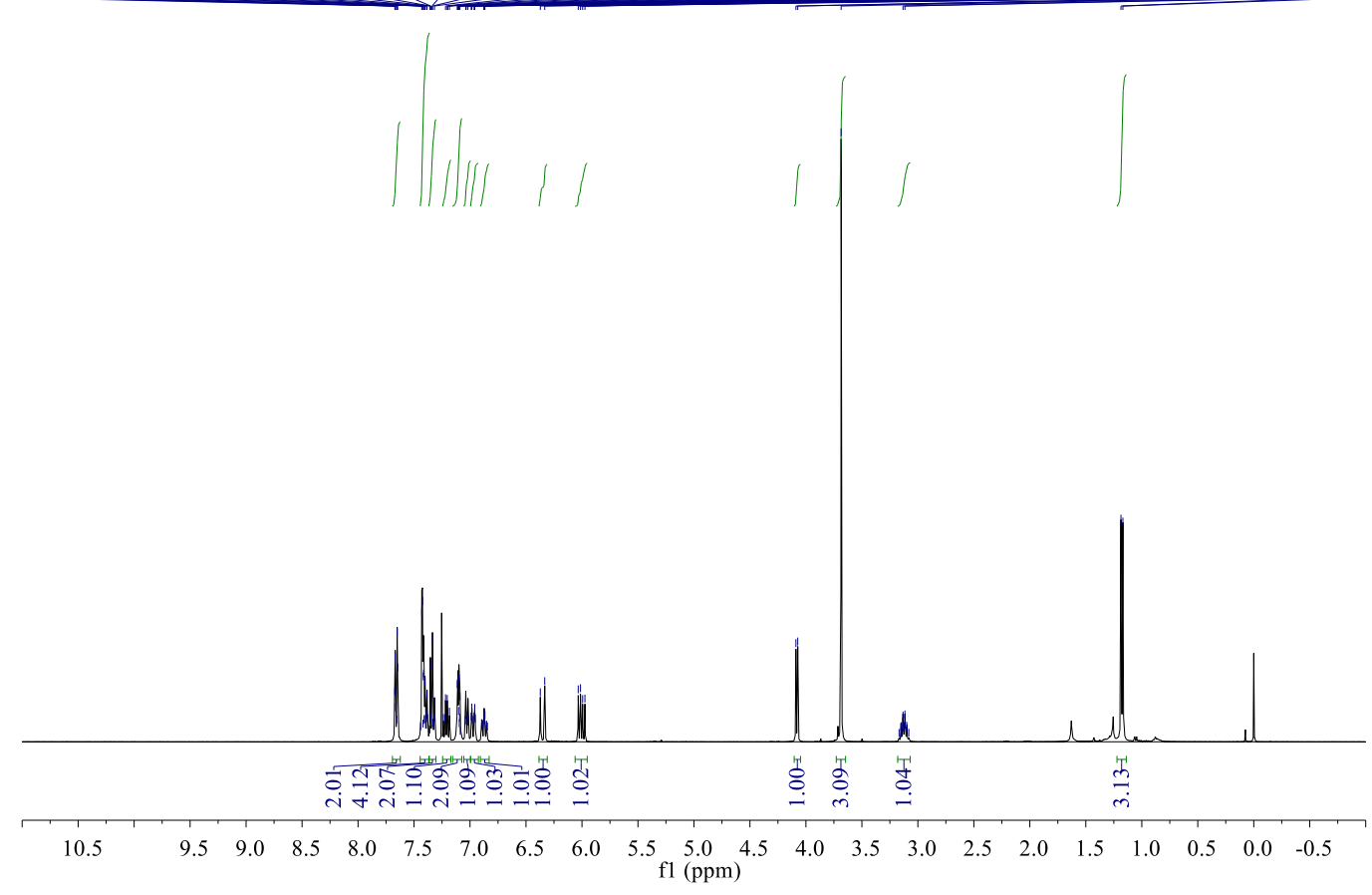

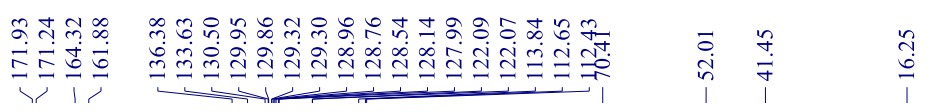
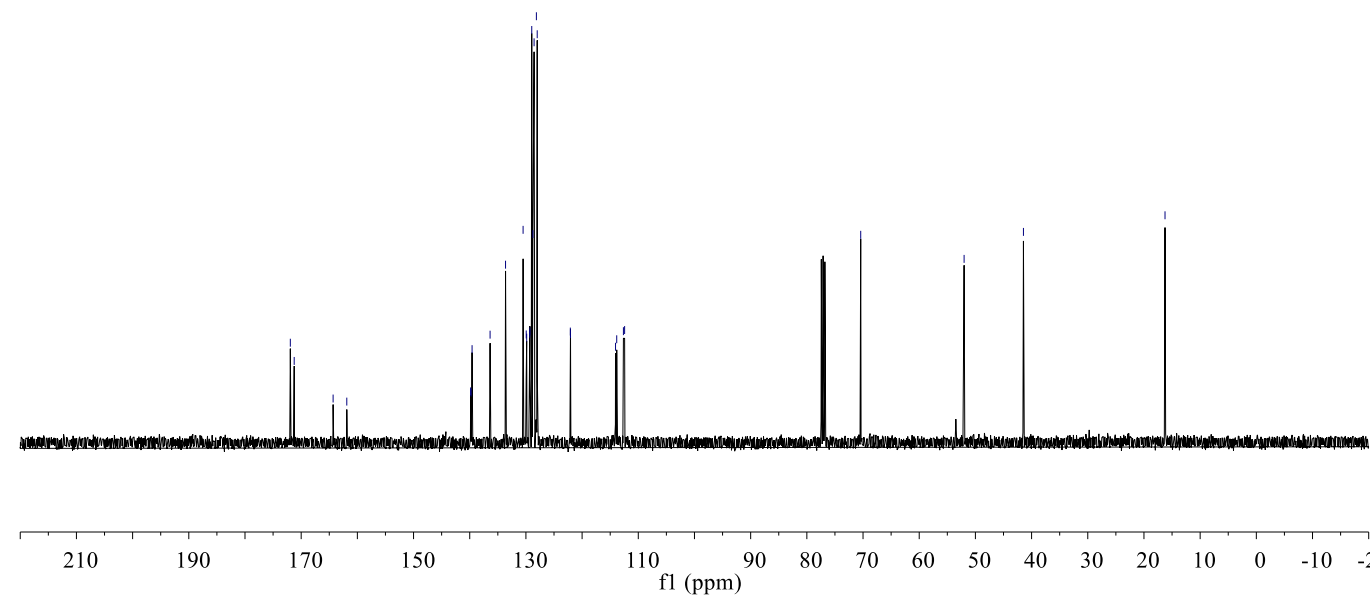


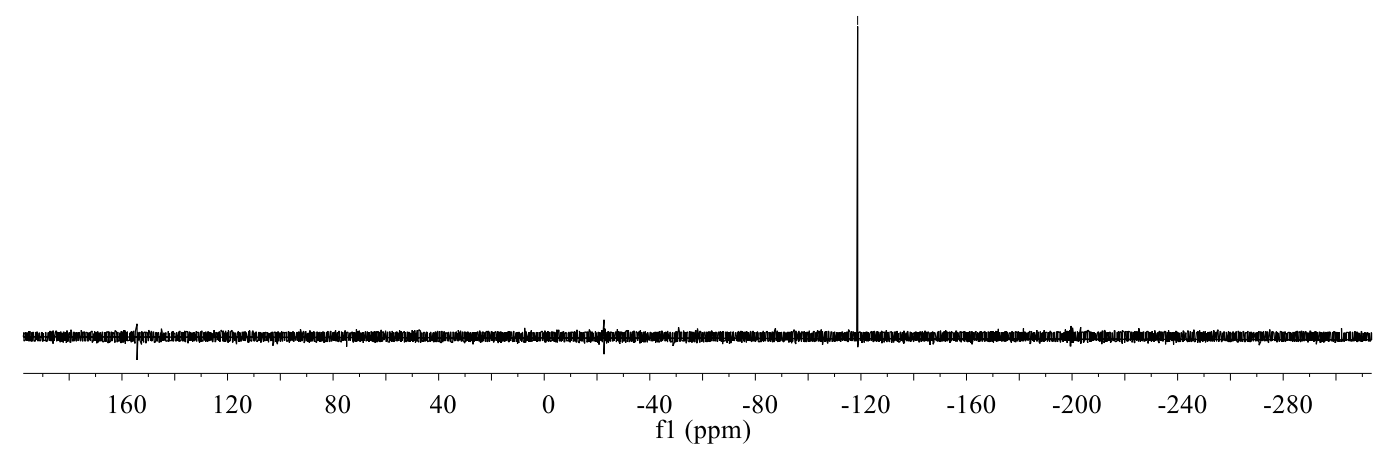<smiles>CC(=O)C(/C=C/c1ccccc1)C(C)/C=N/C(=O)c1ccccc1</smiles>

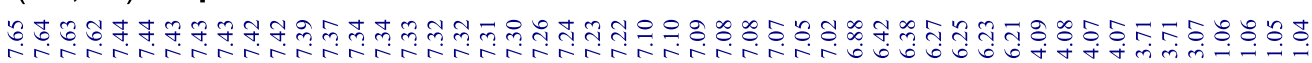

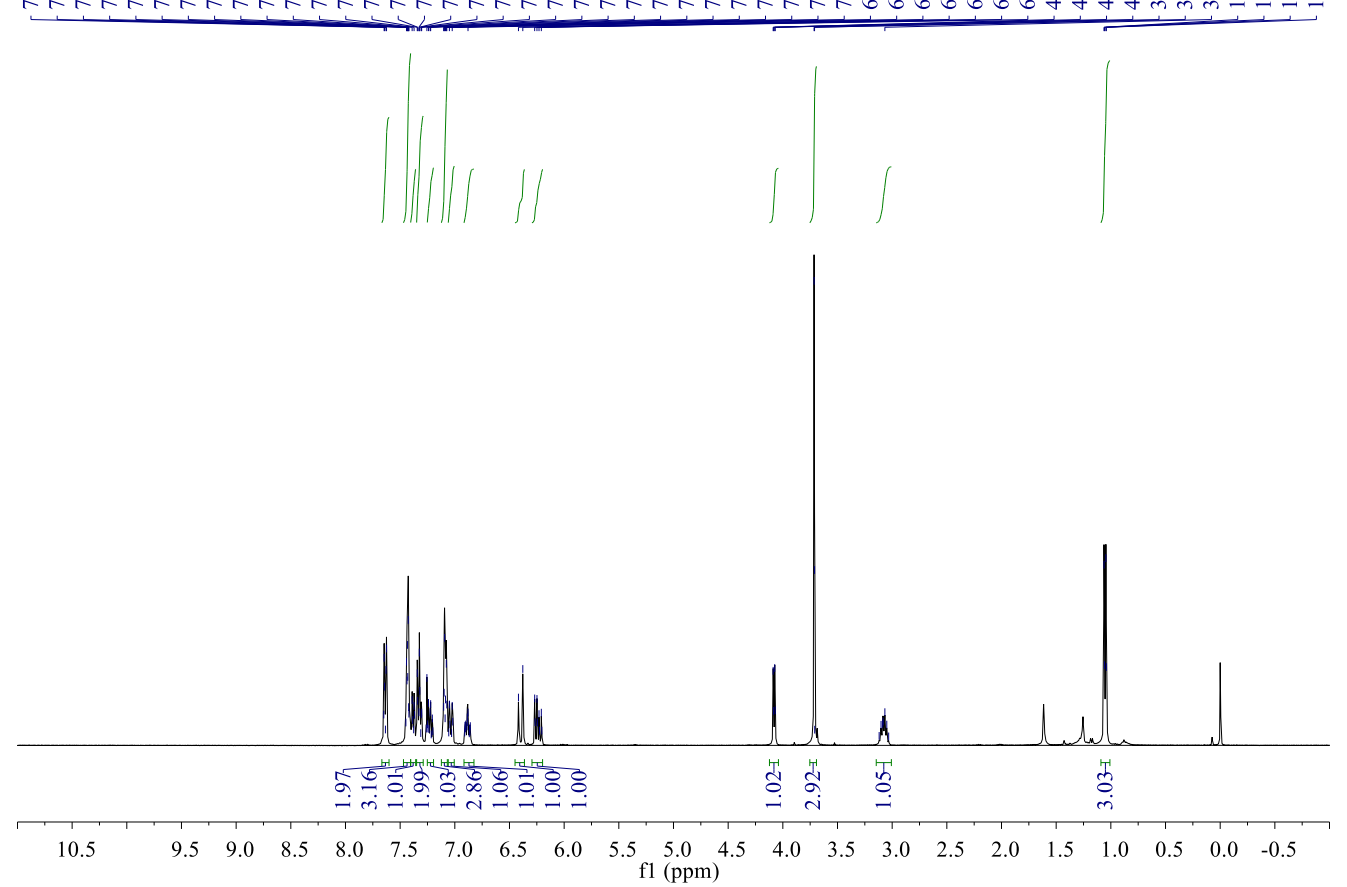




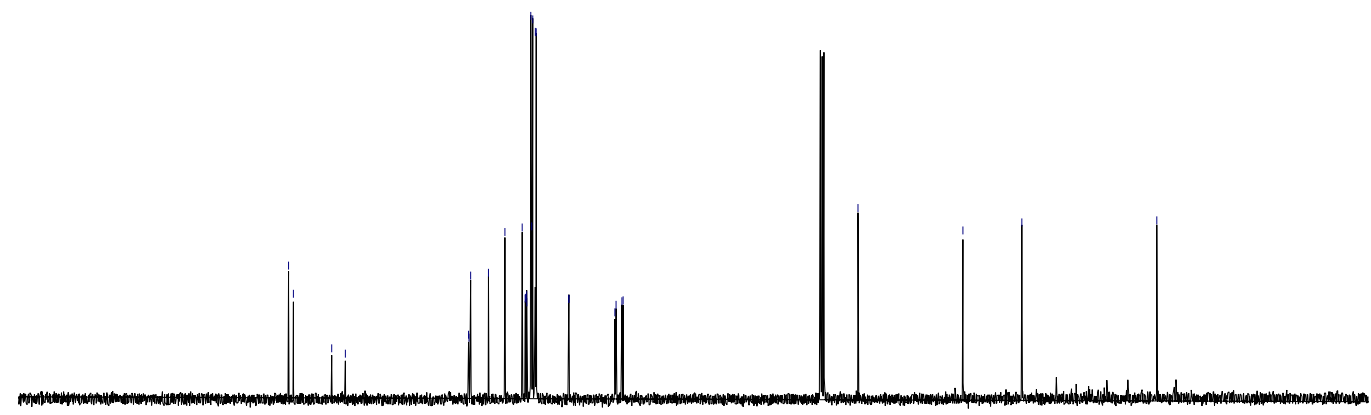

\begin{tabular}{llllllllllllllllll}
\hline 210 & 190 & 170 & 150 & 130 & 110 & 90 & 80 & 70 & 60 & 50 & 40 & 30 & 20 & 10 & 0 & -10 & -
\end{tabular}

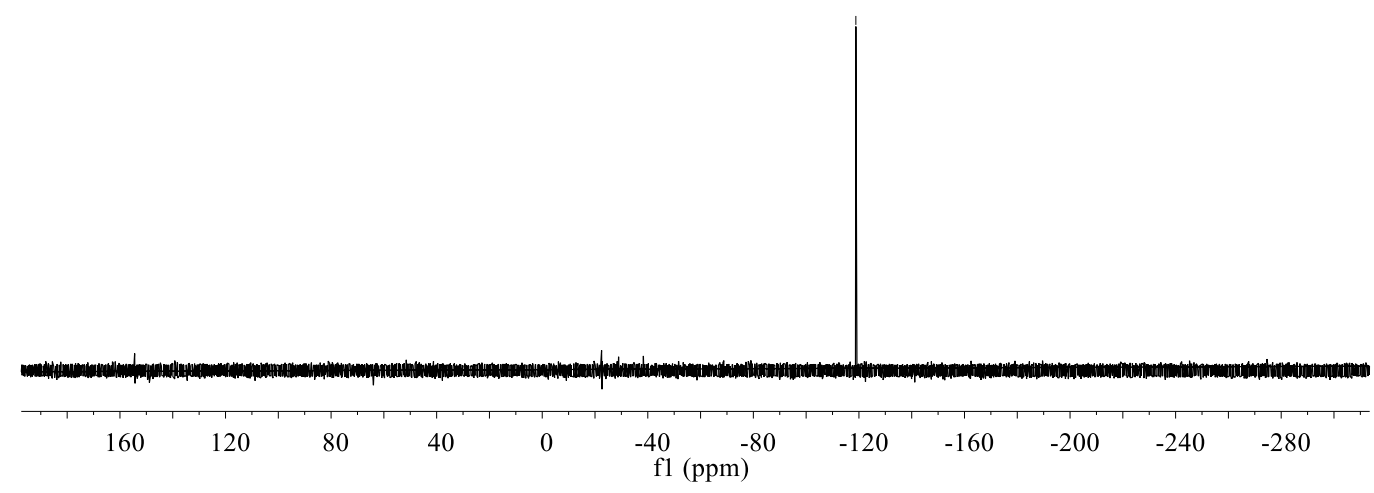

